Florida International University FIU Digital Commons

3-31-2004

\title{
Analysis and design of tall concrete buildings : an investigation regarding the use of cracked versus un- cracked moment of inertia
}

Anas Bataineh

Florida International University

DOI: $10.25148 /$ etd.FI14050455

Follow this and additional works at: https://digitalcommons.fiu.edu/etd

\section{Recommended Citation}

Bataineh, Anas, "Analysis and design of tall concrete buildings : an investigation regarding the use of cracked versus un-cracked moment of inertia" (2004). FIU Electronic Theses and Dissertations. 1431.

https://digitalcommons.fiu.edu/etd/1431 
Miami, Florida

ANALYSIS AND DESIGN OF TALL CONCRETE BUILDINGS: AN INVESTIGATION REGARDING THE USE OF CRACKED VERSUS UN-CRACKED

MOMENT OF INERTIA

A thesis submitted in partial fulfillment of the

requirements for the degree of

MASTER OF SCIENCE

in

CIVIL ENGINEERING

by

Anas Bataineh 
To: Dean Vish Prasad

College of Engineering

This thesis, written by Anas Bataineh, and entitled Analysis and Design of Tall Concrete Buildings: An Investigation Regarding the Use of Cracked versus Un-Cracked Moment of Inertia, having been approved in respect to style and intellectual content, is referred to you for judgment.

We have read this thesis and recommend that it be approved.
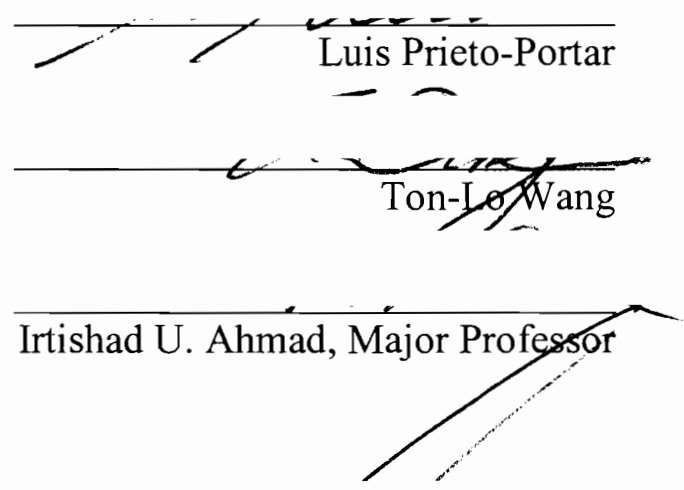

Date of Defense: March 31, 2004

The thesis of Anas Bataineh is approved.

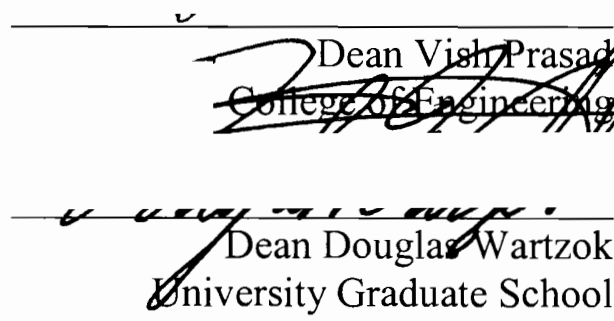

Florida International University, 2004 


\section{DEDICATION}

This thesis is dedicated to my family who always raised me with the strong values of persistence and dedication to always perform to the best of my efforts. To my sister and my brother-in-law who provided support and guidance throughout this crucial time, which will define my future. 


\section{ACKNOWLEDGMENTS}

I would like to express my sincere gratitude to my major professor, Dr. Irtishad Ahmad, P.E., for his guidance and advice during my graduate study at FIU.

I am grateful to Mr. Mehdi Ashraf, P.E., for inspiring me about the research topic and encouraging me to do my best in perusing this research. I am indebted to Professor Luis Prieto-Portar, Ph.D., P.E. and Professor Ton-Lo Wang, Ph.D., P.E., members of my thesis committee, for their continuous support and advice during the research study.

Finally, I would like to express my appreciation to the staff of the Department of Civil and Environmental Engineering for their help and kindness 


\begin{abstract}
OF THE THESIS
ANALYSIS AND DESIGN OF TALL CONCRETE BUILDINGS: AN

INVESTIGATION REGARDING THE USE OF CRACKED VERSUS UN-CRACKED

MOMENT OF INERTIA
\end{abstract}

by

Anas Bataineh

Florida International University, 2004

Miami, Florida

Professor Irtishad U. Ahmad, Major Professor

A debate is currently prevalent among the structural engineers regarding the use of cracked versus un-cracked moment of inertia of the structural elements in analyzing and designing tall concrete buildings. (The basic definition of a tall building, according to the Journal of Structural Design of Tall Buildings Vol. 13, No. 5. 2004 is a structure that is equal to or greater than 160 feet in height, or 6 stories or greater.) The controversy is the result of differing interpretations of certain ACI (American Concrete Institute) code provisions. The issue is whether designers should use cracked moment of inertia in order to estimate lateral deflection and whether the computed lateral deflection should be used to carry out subsequent second-order analysis (analysis considering the effect of firstorder lateral deflections on bending moment and shear stresses). On one hand, bending moments and shear forces estimated based on un-cracked moment of inertia of the sections may result in conservative designs by overestimating moments and shears. On the other hand, lateral deflections may be underestimated due to the same analyses resulting in unsafe designs. 
I. INTRODUCTION

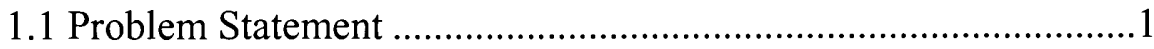

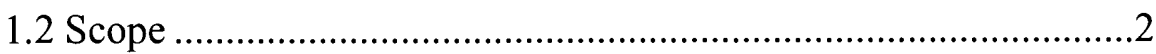

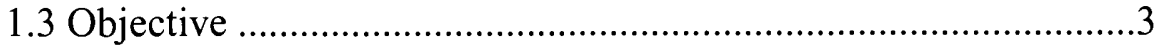

II. BACKGROUND

2.1 ACI Method: Summary...............................................................

2.2 Description of ACI Code Procedure ..............................................8

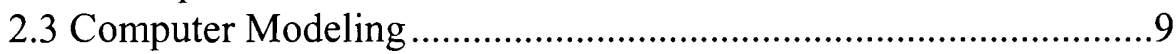

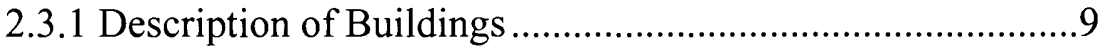

2.3.2 Description of the computer analysis program ....................12

III. METHODOLOGY

3.1 Description of Analysis \& Design Procedure ..................................16

3.2 Description of the MathCAD Sheet used for design .......................18

IV. RESULTS

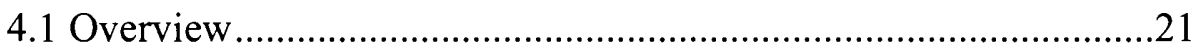

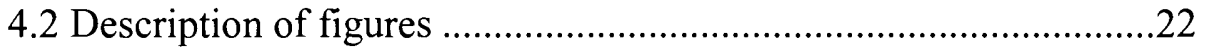

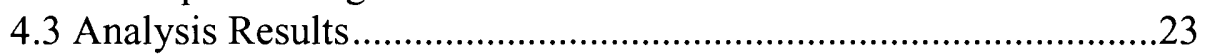

V. CONCLUSIONS AND RECOMMENDATIONS

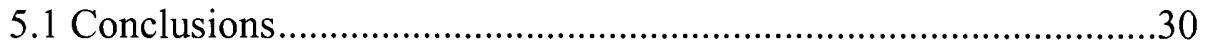

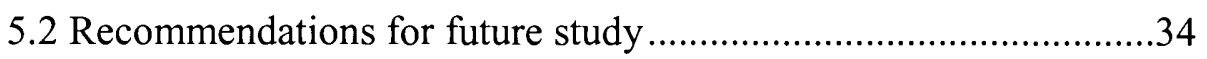

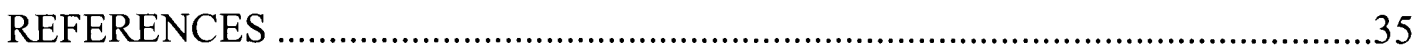

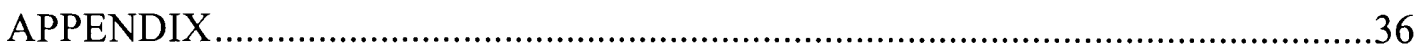




\section{LIST OF FIGURES}

FIGURE

PAGE

2.3.1.1a Elevation view of the typical building used in 10 this research.

2.3.1.1b Plan view of the typical building used in 11 this research.

2.3.2.1 Forces acting on a cable element

4.3.1 ACI sway magnifier factors vs. the ratio of second .23 order to first order moments of the $18 \mathrm{ft}$ story height building.

4.3 .2 ACI non-sway magnifier factors vs. the ratio of second .23 order to first order moments of the $18 \mathrm{ft}$ story height building.

4.3.3 Percentile increase in the square cross- section dimension .24 of a column to equate the un-cracked first order to the cracked second order lateral deflections with respect to story (18 ft story height building).

4.3 .4 ACI sway magnifier factors vs. the ratio of second order .24 to first order moments of the $24 \mathrm{ft}$ story height building.

4.3.5 ............ ACI non-sway magnifier factors vs. the ratio of second ................25 order to first order moments of the $24 \mathrm{ft}$ story height building.

4.3.6 ............ Percentile increase in the square cross- section dimension .25 of a column to equate the un-cracked first order to the cracked second order lateral deflections with respect to story ( $24 \mathrm{ft}$ story height building).

4.3.7 ACI sway magnifier factors vs. the ratio of second order .26 to first order moments of the $30 \mathrm{ft}$ story height building. ACI non-sway magnifier factors vs. the ratio of second .26 order to first order moments of the $30 \mathrm{ft}$ story height building. 
4.3.9 ............ Percentile increase in the square cross- section dimension of a column to equate the un-cracked first order to the cracked second order lateral deflections with respect to story (30 ft story height building).

4.3.10

ACI sway magnifier factors vs. the ratio of second order to first order moments of the $36 \mathrm{ft}$ story height building.

4.3.11 ........... ACI non-sway magnifier factors vs. the ratio of second 28 order to first order moments of the $36 \mathrm{ft}$ story height building.

4.3.12 ............ Percentile increase in the square cross- section dimension .28 of a column to equate the un-cracked first order to the cracked second order lateral deflections with respect to story (36 ft story height building).

4.3.13 ........... Percentile increase in the square cross- section dimension .29 of a column to equate the un-cracked first order to the cracked second order lateral deflections with respect to story (all buildings). 


\section{LIST OS SYMBOLS}

$\Phi$ : Strength reduction factor.

$\Delta_{o}$ : Lateral displacement due to ultimate lateral forces.

$\delta_{n s}$ : ACI code nonsway moment magnification factor.

$\delta_{s}:$ ACI code sway moment magnification factor.

$D_{m}$ : percentile difference between the first and second analyses sway moments.

$D_{n s 2}:$ percentile difference between the first and second analyses gravity moments.

$h$ : the dimension of the column square cross-section.

$I_{c r e}$ : Effective moment of inertia of the column cracked section.

$I_{g e}$ : Effective moment of inertia of the column gross section.

$k$ : Effective length factor.

$M_{u}$ : Ultimate Bending Moment.

$P_{c}$ : Critical buckling load.

$P_{u}$ : Ultimate axial load.

$Q$ : Stability index.

$V_{u}$ : Ultimate shear force. 


\section{CHAPTER 1}

\section{INTODUCTION}

\subsection{PROBLEM STATEMENT:}

A debate is currently prevalent among the structural engineers regarding the use of cracked versus un-cracked moment of inertia of the structural elements in analyzing and designing tall concrete buildings. The controversy is the result of differing interpretations of certain ACI (American Concrete Institute) code provisions. The issue is whether designers should use cracked moment of inertia in order to estimate lateral deflection and whether the computed lateral deflection should be used to carry out subsequent second-order analysis (analysis considering the effect of first-order lateral deflections on bending moment and shear stresses). On one hand, bending moments and shear forces estimated, based on un-cracked moment of inertia, may result in conservative designs by overestimating moments and shears. On the other hand, lateral deflections may be underestimated due to the same analyses resulting in unsafe designs. The ACI code provisions leave room for widely differing predictions and do not provide clear guidelines. 


\subsection{SCOPE:}

The scope of this investigation is limited to determining theoretically the behavior of columns in tall concrete buildings under lateral forces. A commercially available computer software package, employing the finite element method (FEM) is used to carry out the analytical investigation. Four buildings with different story heights are modeled, each has the same structural configuration (one bay by one bay), material properties and number of stories (30 stories).

The structural configuration of the buildings is chosen based on the following:

1) Each one of the buildings satisfies the definition of the tall building.

2) The story height of each building is different; different lateral deflections and moments will be induced causing different $P$-Delta (second order) effects.

3) All the structural elements in the buildings are designed according to ACI Code provisions.

Three types of analyses are performed for each building, un-cracked first order, uncracked second order $(P$-Delta $)$ and cracked second order $(P-$ Delta $)$. The results are used to investigate the ACI Code moment magnification factors and the behavior of columns in concrete buildings. 


\subsection{OBJECTIVES:}

1) To investigate the applicability of ACI Code moment magnifier method in terms of slenderness ratio $\left(k L_{u} / r\right)$ ranges for braced and sway columns (unbraced).

2) To investigate behavior of tall concrete buildings analytically under lateral force effects with different story heights based on cracked and un-cracked moment of inertia of the structural members sections.

3) To develop an improved procedure for analysis taking realistic behavior of tall concrete buildings under ultimate loads (gravity \& lateral) into account.

4) To develop graphical design aids for assisting structural engineers. 


\section{CHAPTER 2}

\section{BACKGROUND}

\subsection{ACI METHOD: SUMMARY}

Tall or high-rise buildings are typically statically indeterminate structures. That means, bending moments and shear forces induced in the structural elements (mainly beams and columns) are dependent on deflections (work done by the external loads) in these members, and these deflections vary according to the stiffness (moment of inertia) of structural elements. The structural elements are designed to carry the ultimate applied loads; these loads will force the structure to deform in a way to reach the stable condition (work done by external loads equals to the work done by internal loads). Accordingly, the flexural rigidity of the structural elements will be decreased due to cracking of the sections; further deformations will be induced because of the increase in flexibility of that structure.

First, the study was conducted by going through ACI code provisions to investigate the methodology suggested in $\mathrm{ACI}$ research to come up with the moment magnifier method and the underlying assumptions that led to this methodology.

Section 10 in ACI code 2002 was the main reference to this research investigation, all of the variables controlling the analysis and design processes were studied in depth in order to understand the rationale used by ACI.

In $A C I$ R10.13.3 it is mentioned that the analysis described in moment magnifier method deals only with plane frames subjected to loads causing deflections in that plane. If torsional displacements are significant, a three-dimensional second-order analysis is suggested. In this research only symmetrical buildings in geometry and loading are 
considered. Thus no torsional displacements are significant in any building used in this research.

In $A C I R 10.13 .4 .1$ it is mentioned that a second-order analysis is a frame analysis that includes the internal force effects resulting from deflections. When a second-order elastic analysis is used to compute $\delta_{s} M_{s}$, magnified moments due to lateral loads, and the deflections should be representative of the stage immediately prior to the ultimate load. For this reason the reduced $E_{c} I_{g}$ values given in 10.11.1 should be used in the secondorder analysis.

(b) Moments of inertia

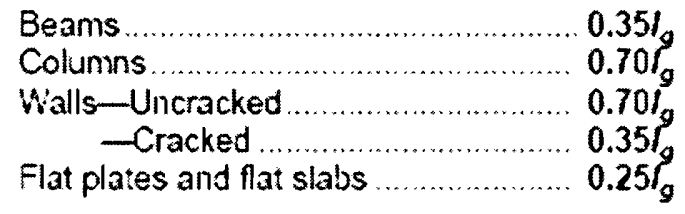

$\mathrm{ACI}$ uses a factor called $\beta_{d}$ to account for cracking in structural members due to creep and shrinkage. The term $\beta_{d}$ is defined differently for non-sway and sway.

$$
\begin{aligned}
& \boldsymbol{\beta}_{d}=(a) \text { for nonsway frames, } \boldsymbol{\beta}_{d} \text { is the ratio of the } \\
& \text { maximum factored axial sustained load to } \\
& \text { the maximum factored axial load associ- } \\
& \text { ated with the same load combination; } \\
& \text { (b) for sway frames, except as required in (c) } \\
& \text { of this definition, } \beta_{d} \text { is the rato of the maxi- } \\
& \text { mum factored sustained shear within a story } \\
& \text { to the maximum factored shear in that story: } \\
& \text { (c) for stability checks of sway frames carred } \\
& \text { out in accordance with } 10.13,6, \beta_{d} \text { is the ratio } \\
& \text { of the maximum factored sustained axial load } \\
& \text { to the maximum factored axial load }
\end{aligned}
$$

In this research no shear walls were used, shear walls takes all of the lateral loads applied to the concrete building and the main function of the other structural members will be to carry the gravity loads only. So, the stability index $(Q)$ was used to distinguish between a braced story and a sway story, $A C I$ 10.11.4.2 was used: 
10.11.4.2 - It also shall be permitted to assume a story within a structure is nonsway if:

$$
Q=\frac{\Sigma P_{u} \Delta_{o}}{V_{u} l_{c}}
$$

is less than or equal to 0.05 , where $\Sigma P_{u}$ and $V_{u}$ are the total vertical load and the story shear, respectively, in the story in question and $\Delta_{0}$ is the first-order relative defection between the top and bottom of that story due to $V_{u}$.

In $A C I$ R10.11.4 it is mentioned that the moment magnifier design method requires the designer to distinguish between non-sway frames, (designed according to 10.12) and sway frames (designed according to 10.13). Frequently this can be done by inspection by comparing the total lateral stiffness of the columns in a story to that of the bracing elements. A compression member may be assumed non-sway by inspection if it is located in a story in which the bracing elements (shear walls, shear trusses, or other types of lateral bracing) have such substantial lateral stiffness to resist the lateral deflections of the story that any resulting lateral deflection is not large enough to affect the column strength substantially. If not readily apparent by inspection, 10.11.4.1 and 10.11.4.2 give two possible ways of doing this. In 10.11.4.1, a story in a frame is said to be non-sway if the increase in the lateral load moments resulting from P-Delta effects does not exceed 5 percent of the first-order moments. 10.28 Section 10.11.4.2 gives an alternative method of determining this based on the stability index for a story $Q$. In computing $Q, \Sigma P_{u}$ should correspond to the lateral loading case for which $\Sigma P_{u}$ is greatest. A frame may contain both non-sway and sway stories. This test would not be suitable if $V_{u}$ is zero. 
$\mathrm{ACI}$ also specifies another limit, in addition to the above, for the use of the moment magnifier method. Slenderness Ration $\left(k L_{\mathrm{u}} / r\right)$ must be less than 100 , otherwise the moment magnifier method is not applicable any more, and all of the columns in all of the three buildings used in this research satisfy that limit.

The effective length factor $(k)$ was calculated according to $A C I 10.12$, taking into account the connection condition for each column and whether the story investigated is Braced or not (sway).

The strength reduction factor $(\phi)$ was calculated for each column in accordance with $A C I$ 9.3.2:

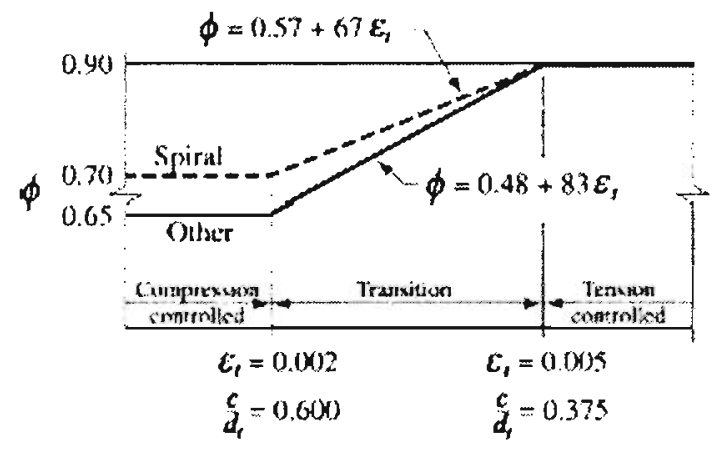

literpolation on cid: Spiral $\phi=0.37+0.20\left(c / d_{t}\right)$ Oincr $\phi=0.23+0.25 /\left(c / d_{s}\right)$

The ACI Code specifies a limit for the compression member steel reinforcement Ratio, with a maximum of $8 \%$ going down to a minimum of $1 \%$ of the gross crosssectional area, in this research a steel reinforcement ration of $1.5 \%$ was used. 


\section{9 - Limits for reinforcement of compression members}

10.9.1 - Area of longitudinal reinforcement for noncomposite compression members shall be not less than 0.01 nor more than 0.08 times gross area $A_{g}$ of section.

\subsection{DESCRIPTION OF ACI CODE PROCEDURE:}

In this section, the $\mathrm{ACI}$ procedure for designing compression members is given:

1. Specify which materials properties will be used in the analysis and design of the structural member, $f_{c}^{\prime}$ (concrete compressive strength), $\gamma$ (unit weight of concrete), $f_{y}$ (grade of reinforcing steel).

2. Assume preliminary section dimensions.

3. From the analysis results, using the axial loads, moments and shears for each load case (dead, live, wind... etc), calculate the ultimate load combinations. And find which combination is the most critical for design.

4. Determine whether the story is sway or non-sway using the ACI limits for the stability index $(Q)$.

5. calculate the effective length factor using the equations/charts provided by $\mathrm{ACI}$, then calculate the slenderness ratio and find out if the member is slender or not. Make sure $\left(k L_{u} / r\right.$ is less that 100$)$.

6. calculate the magnifier factors:

a) If the member is non-sway and short: no magnifications of moments are required.

b) If the member is non-sway and Slender: gravity load combination should be magnified by $\delta_{n s}$. 
c) If the member is sway and short: no magnifications of moments are required.

d) If the member is sway and Slender: gravity load combination must be magnified by $\delta_{n s}$ if $\left[L_{u} / r>35 /\left(P_{u} / f_{c}^{\prime} A_{g}\right)^{\wedge} 0.5\right]$ and the sway moments must be magnified by $\delta_{s}$.

e) If the member is sway and Slender: if $\left[L_{w} / r<35 /\left(P_{u} / f_{c}^{\prime} A_{g}\right)^{\wedge} 0.5\right]$ no magnification is required for gravity load combination and the sway moments must be magnified by $\delta_{s}$.

7. Calculate the Design moment, magnified ultimate moment, and the ultimate axial load.

8. Calculate the actual eccentricity and the minimum eccentricity specified by $\mathrm{ACI}$ code and take which ever is the greater.

9. Calculate the nominal axial load and moment by dividing the forces from step (7) with the strength reduction factor.

10. Using the interaction diagrams or equations solving find out the required steel ratio.

11. Check if the section is adequate, if not go back to step 2 and increase the section dimensions.

\subsection{COMPUTER MODELING:}

\subsubsection{DESCRIPTION OF BUILDINGS:}

The typical building used for analysis in this research is described below:

1) Thirty Story building.

2) Four building with four different story heights: $18 \mathrm{ft}, 24 \mathrm{ft}, 30 \mathrm{ft}$ and $36 \mathrm{ft}$. 
3) One bay by one bay of $24 \mathrm{ft}$ span.

4) Each story consists of 4 corner columns, 4 beams and a concrete slab connecting them.

5) The applied loads on the concrete slab are $20 \mathrm{psf}$ dead and $80 \mathrm{psf}$ live load.

6) The applied lateral load on the columns is the wind load with a speed of $150 \mathrm{MPH}$.

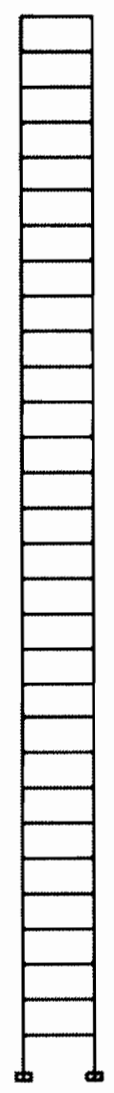

Figure 2.3.1.1a

Elevation view of the typical building used in this research 


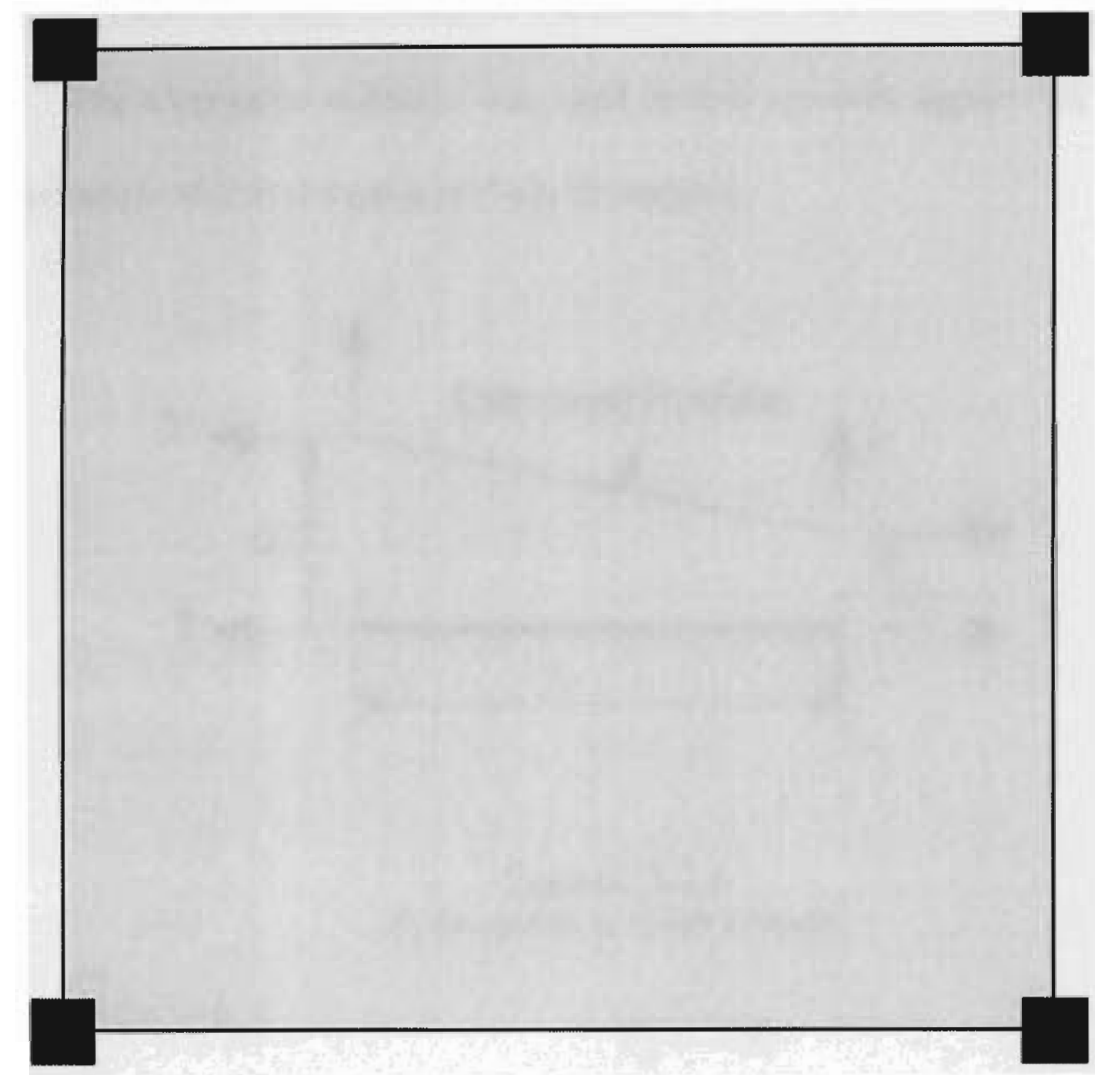

Figure 2.3.1.1b

Plan view of the typical building used in this research 


\subsubsection{DESCRIPTION OF THE COMPUTER ANALYSIS PROGRAM:}

The Computer software was used in this research applies the concept of the geometric stiffness matrix in P-Delta analysis.

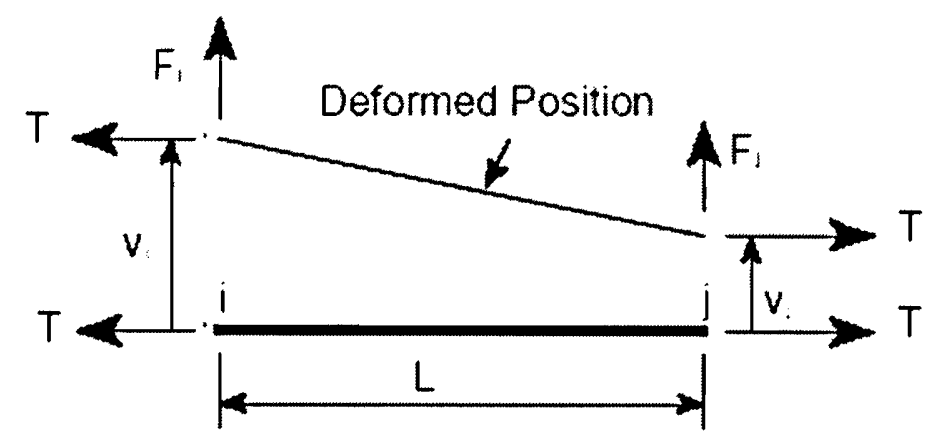

Figure 2.3.2.1

Forces acting on a cable element

$$
\begin{array}{lc}
F_{i}=\frac{T}{L}\left(v_{i}-v_{j}\right) & F_{j}=-F_{i} \\
{\left[\begin{array}{l}
F_{i} \\
F_{j}
\end{array}\right]=\frac{T}{L}\left[\begin{array}{cc}
1 & -1 \\
-1 & 1
\end{array}\right]\left[\begin{array}{l}
v_{i} \\
v_{j}
\end{array}\right] \quad \text { or symbolically. } \quad \mathbf{F}_{\mathbf{g}}=\mathbf{k}_{\mathbf{g}} \mathbf{v}}
\end{array}
$$

The Geometric Stiffness matrix $\left(\boldsymbol{k}_{G}\right)$ :

$$
\left[\begin{array}{c}
F_{i} \\
M_{i} \\
F_{3} \\
M_{3}
\end{array}\right]=\frac{T}{30 L}\left[\begin{array}{cccc}
36 & 3 L & -36 & 3 L \\
3 L & 4 L^{2} & -3 L & -L^{2} \\
-36 & -3 L & 36 & -3 L \\
3 L & -L^{2} & -3 L & 4 L^{2}
\end{array}\right]\left[\begin{array}{l}
v_{i} \\
\phi_{i} \\
v_{j} \\
\phi_{j}
\end{array}\right]
$$$$
\text { or. } \mathbf{F}_{\hat{o}}=\mathbf{k}_{G} \mathbf{v}
$$

The Elastic Stiffness without Shear Deformations: 


$$
\left[\begin{array}{c}
F_{i} \\
M_{i} \\
F_{j} \\
M_{j}
\end{array}\right]=\frac{E I}{L^{3}}\left[\begin{array}{cccc}
12 & 6 L & -12 & 6 L \\
6 L & 4 L^{2} & -6 L & -2 L^{2} \\
-12 & -6 L & 12 & -6 L \\
-6 L & -2 L^{2} & -6 L & 4 L^{2}
\end{array}\right]\left[\begin{array}{c}
v_{j} \\
\phi_{i} \\
v_{j} \\
\phi_{j}
\end{array}\right] \quad \text { or. } F_{E}=\mathrm{k}_{\bar{E}} \mathrm{r}
$$

The Total stresses acting on a member are:

$$
\mathbf{F}_{I}=\mathbf{F}_{E}+\mathbf{F}_{G}=\left[k_{E}+k_{G}\right] \mathbf{v}=k_{I} \mathbf{v}
$$

In building analysis the lateral movement of a story mass to a deformed position generates second-order overturning moments. This second-order behavior has been termed the P-Delta effect since the additional overturning moments on the building are equal to the sum of story weights " $P$ " times the lateral displacements "Delta".

Many techniques have been proposed for evaluating this second-order behavior. Some methods consider the problem as one of geometric non-linearity and propose iterative solution techniques that can be numerically inefficient. Also, these iterative methods are not appropriate for dynamic analysis where the $P$ Delta effect causes lengthening of the periods of vibration. However, the simple approach used in their derivation should add physical insight to the understanding of $P$-Delta behavior in buildings.

The procedure involving $P$-Delta analysis can be summarized as follows:

1. Choose geometrical sections of the frame and its columns and their stiffness $(E I)$ by approximate procedure. 
2. Calculate the drifts (later deflections $\Delta_{i}$ ) and the corresponding ultimate loads $\left(P_{u}\right)$ at joints $i=1,2 \ldots n$. (as in the figure).

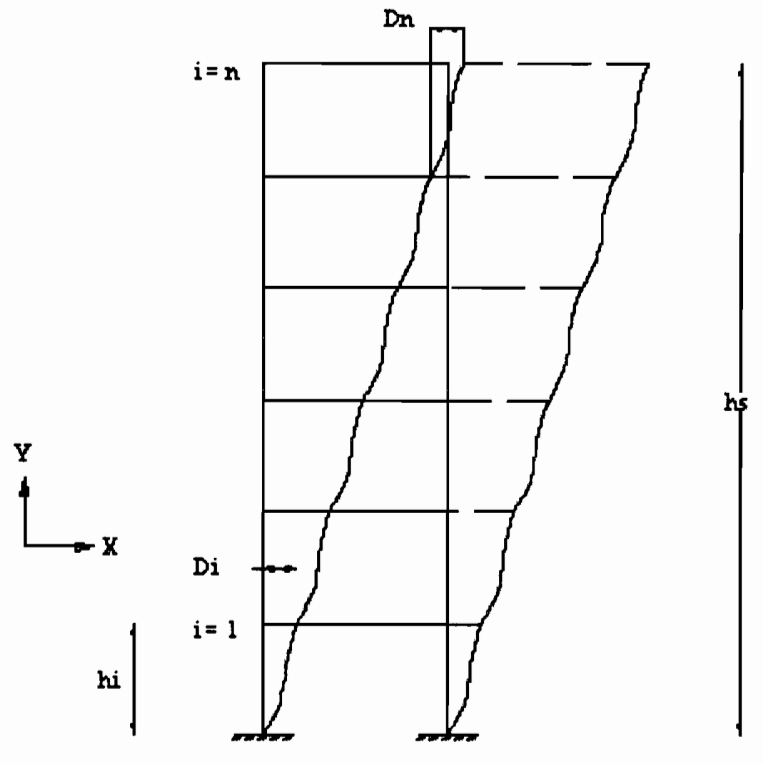

Figure 2.3.2.2

Deformed shape of the typical building used in this research

3. Find the equivalent horizontal forces $\left(H_{i}=P_{i} \Delta i / h_{i}\right)$

4. Add the values obtained in step 3 to the actual lateral loads acting on the frame.

5. Perform a frame analysis using approximate computer program (or hand analysis).

6. The iterative computer program, using the stiffnesses $(E I)$, chosen for the input data gives $\Delta_{\mathrm{i}}$ results that have to be compared with the previous analysis results. 
7. If all $\Delta_{i}$ are less than or equal the ones obtained from the preceding analysis, accept the analysis. If not run more cycles and modify the stifnesses.

The P-Delta problem can be linearized and the solution to the problem can be obtained directly and exactly, without iteration, in building type structures where the weight of the structure is constant during lateral motions and the overall structural displacements can be assumed to be small compared to the structural dimensions.

The method does not require iteration since the total axial force at a story level is equal to the weight of the building above that level and does not change during the application of lateral loads. Therefore, the sum of the column of geometric stiffness terms associated with the lateral loads cancels and only the axial forces due to the weight of the structure need to be included in the evaluation of the geometric stiffness terms for the entire building. 


\section{CHAPTER 3}

\section{METHODOLOGY}

\subsection{DESCRIPTION OF ANALYSIS AND DESIGN PROCEDURE:}

In this section the analysis and design procedure of a typical building is described in detail, including the assumptions made to get the results obtained.

After choosing the structural configuration of the typical building, material properties and dimensions, the building was modeled using the commercially available FEM software. Assuming preliminary sections of the structural members the first order un-cracked analysis was performed. Using the axial loads, moments and shears obtained from that analysis the structural members were design to resist the ultimate loads applied. Then, the new sections were input again to the computer model and the first order uncracked analysis was performed and a new set of member forces was obtained. The structural members were designed again to carry the new forces of the second analysis, if the input sections were different from the output (designed) sections, the analysis operation would be repeated until input sections were within $10 \%$ difference from output sections.

After finishing the design for the first order un-cracked analysis and making sure that all of the structural members would carry the applied loads, a P-Delta un-cracked (second order) analysis was performed to account for the additional moments induced because of the building lateral drifts. Once that is done, another check would be performed for the capacity of the building's structural members; if any member fails to carry its assigned loads the section of that member must be redesigned. 
Using the design results obtained (sections dimensions, depth of the compression block of the section and the longitudinal area of the reinforcing steel), the effective cracked and the effective gross moments of inertia of each section is calculated and a ratio of cracked to un-cracked moments of inertia were calculated. These ratios would be entered into the computer model to modify each member's stiffness. A P-Delta (second order) analysis of the modified building is performed and a new set of results would be obtained (the structural members would pass the strength capacity check but the lateral drifts for each story would be increased).

The section of each structural member would be modified in a way to balance the lateral drifts of the first order un-cracked analysis with the second order (P-Delta) cracked analysis. That was done by increasing the sections as a function of the increase in the lateral drifts of each story.

Sets of graphs comparing the results obtained were drawn for each one of the four buildings and conclusions were made regarding the behavior of the buildings described in these graphs. 


\subsection{DESCRIPTION OF THE MATHCAD SHEET USED FOR DESIGN:}

A MathCAD sheet was created to facilitate the process of design and comparing the results between the three types of analysis were performed for each building.

Here is a summary of the input and output obtained from the MathCAD sheet:

Input:

1) First order and second order axial loads, moments, shears and lateral deflections of each story (dead, live and wind) were exported from the analysis software to Microsoft excel then as an input file to MathCAD.

2) The MathCAD sheet will calculate the ultimate loads applied to the structural member and the moment's modification factors to prepare the process of design.

3) The reinforcing steel ratio can be changed in the sheet; a value of $1.5 \%$ was used throughout in this research.

\section{Output:}

1) Section dimensions.

2) Unsupported length of column.

3) Area of longitudinal reinforcing steel.

4) Effective length factor $(k)$.

5) Slenderness Ratio $\left(k L_{u} / r\right)$.

6) Whether the story is sway or non-sway (braced).

7) Whether the columns of each story are slender or short.

8) Stability Index $(Q)$.

9) Non-sway moment magnification factor.

10) Sway moment magnification factor. 
11) Capacity ratios of the section.

12) Compression block dimension (c).

13) Strength Reduction Factor $(\phi)$.

14) Difference in first order and second order $(P$-Delta $)$ non-sway moments.

15) Difference in first order and second order $(P$-Delta $)$ sway moments.

16) Effective cracked moment of inertia $\left(I_{c r e}\right)$.

17) Effective gross moment of inertia $\left(I_{g e}\right)$.

18) The ratio of cracked to un-cracked moments of inertia.

The MathCAD sheet applies the design provisions of ACI Code for compression members; here is the process of design using the MathCAD sheet:

1) Importing the output file from the Computer's software analysis results.

2) Calculating the ultimate loads applied to the structural member.

3) Calculating the stability index of the story.

4) Determining of the story is braced or sways.

5) Calculating the creep factor for the situation applied.

6) Calculating the relative stiffnesses of the column-beam connection.

7) Calculating the effective length factor.

8) Calculating the slenderness ratio.

9) Determining if the structural member is short or slender.

10) Calculating the critical buckling load.

11) Calculating the non-sway moment magnification factor.

12) Calculating the sway moment magnification factor.

13) Calculating the design ultimate axial load, moment and eccentricity. 
14) Performing the design process to carry the ultimate loads.

15) Calculating capacity ratios, stresses in compression and tension steels and the strength reduction factor.

16) Calculating the differences in first order and second order analysis results.

17) Calculating the effective cracked and gross moments of inertia.

18) Calculating the ratio of cracked to un-cracked moments of inertia.

19) Exporting the results to an excel output file to perform the output analysis in excel. 


\section{CHAPTER 4}

\section{RESULTS}

\subsection{OVERVIEW:}

After finishing the analysis and design processes and calculating the output parameters of interest in each building, a set of three graphs was drawn to compare the research results with the $\mathrm{ACI}$ code recommendations.

The first graph compares the sway moment modification factor with the real difference in the un-cracked first order and second order analyses sway moments with respect to the varying slenderness ratio for each story of the building.

The second graph compares the non-sway modification factor with the real difference in the un-cracked first order and second order analyses non-sway moments with respect to the varying slenderness ratio for each story of the building.

The third graph gives an indication of the increase in the cross-sectional dimensions from the first order un-cracked analysis to the second order (P-Delta) cracked analysis to get the same lateral deflections with respect to each story. 


\subsection{DESCRIPTION OF FIGURES:}

For each building, a set of three graphs is shown:

1) Sway stories:

A comparison graph represents the ACI sway magnification factors $\left(\delta_{s}=\frac{1}{1-Q}\right.$ or $\frac{1}{1-\frac{\sum P_{u}}{0.75 \sum P_{c}}}$ ) versus the percentage difference from first order sway moment to second order sway moment $\left(D_{m}\right)$.

2) Braced stories:

A comparison graph represents the ACI nonsway magnification factors $\left(\delta_{n s}=\frac{1}{1-\frac{P_{u}}{0.75 P_{c}}}\right)$ versus the percentage difference from first order gravity moment to second order gravity moment $\left(D_{n s 2}\right)$.

3) The percentage increase in the square column dimension $(h)$ to equate the first order uncracked lateral deflections to the second order cracked lateral deflections versus story. 


\subsection{ANALYSIS RESULTS:}

1) The $18 \mathrm{ft}$ story height building:

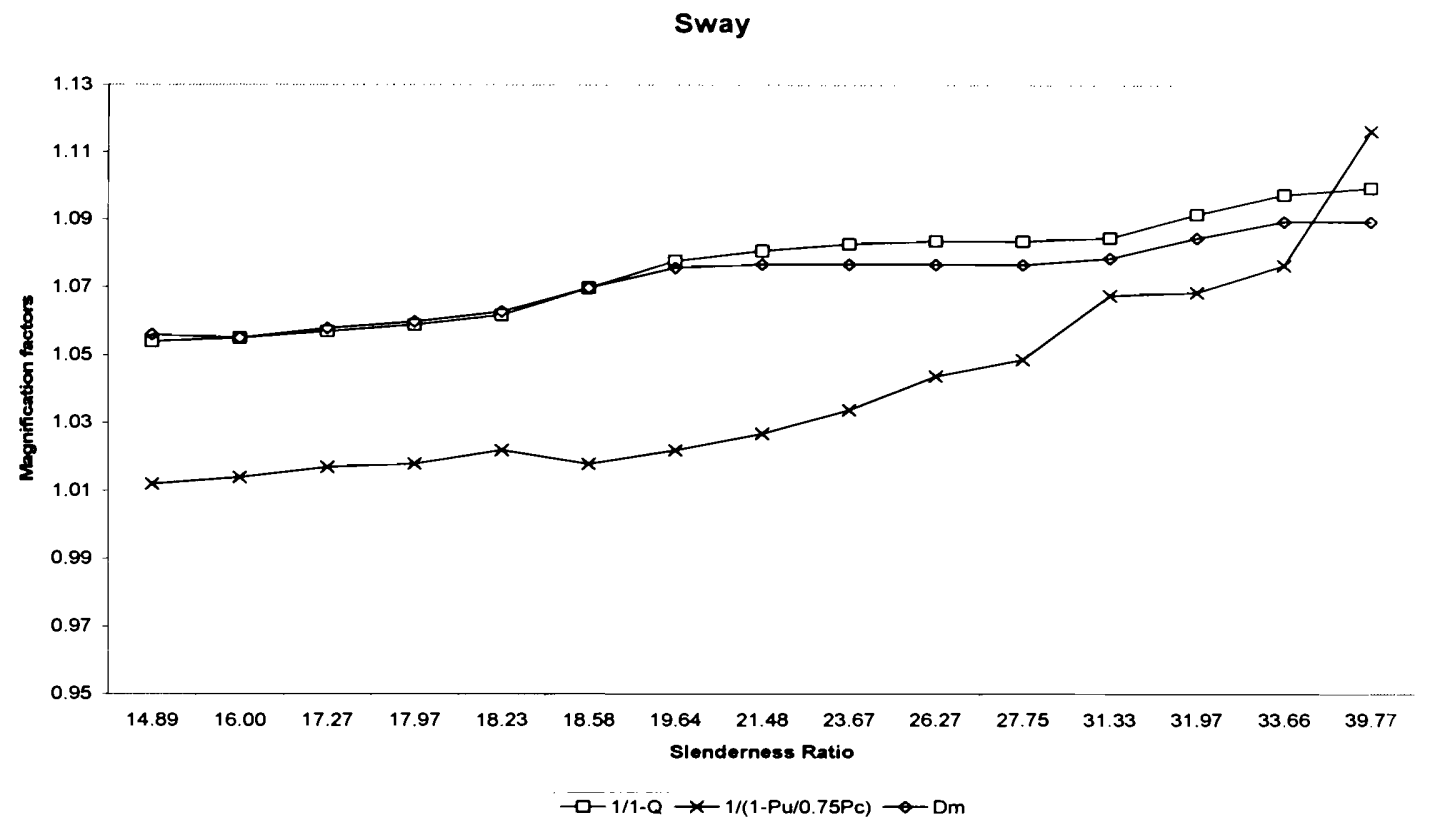

Figure 4.3.1

ACI sway magnifier factors vs. the ratio of second order to first order moments

Braced

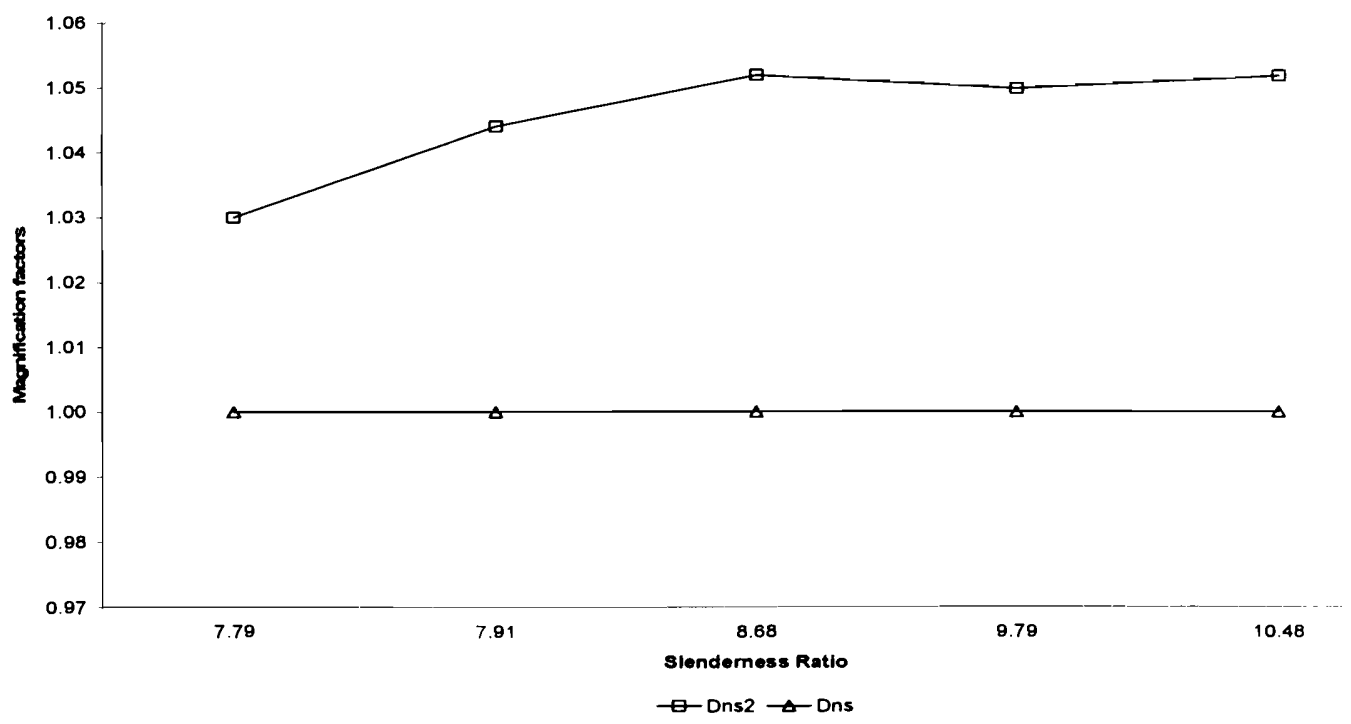

Figure 4.3.2

ACI non-sway magnifier factors vs. the ratio of second order to first order moments 
$h$ diff $(\%)$

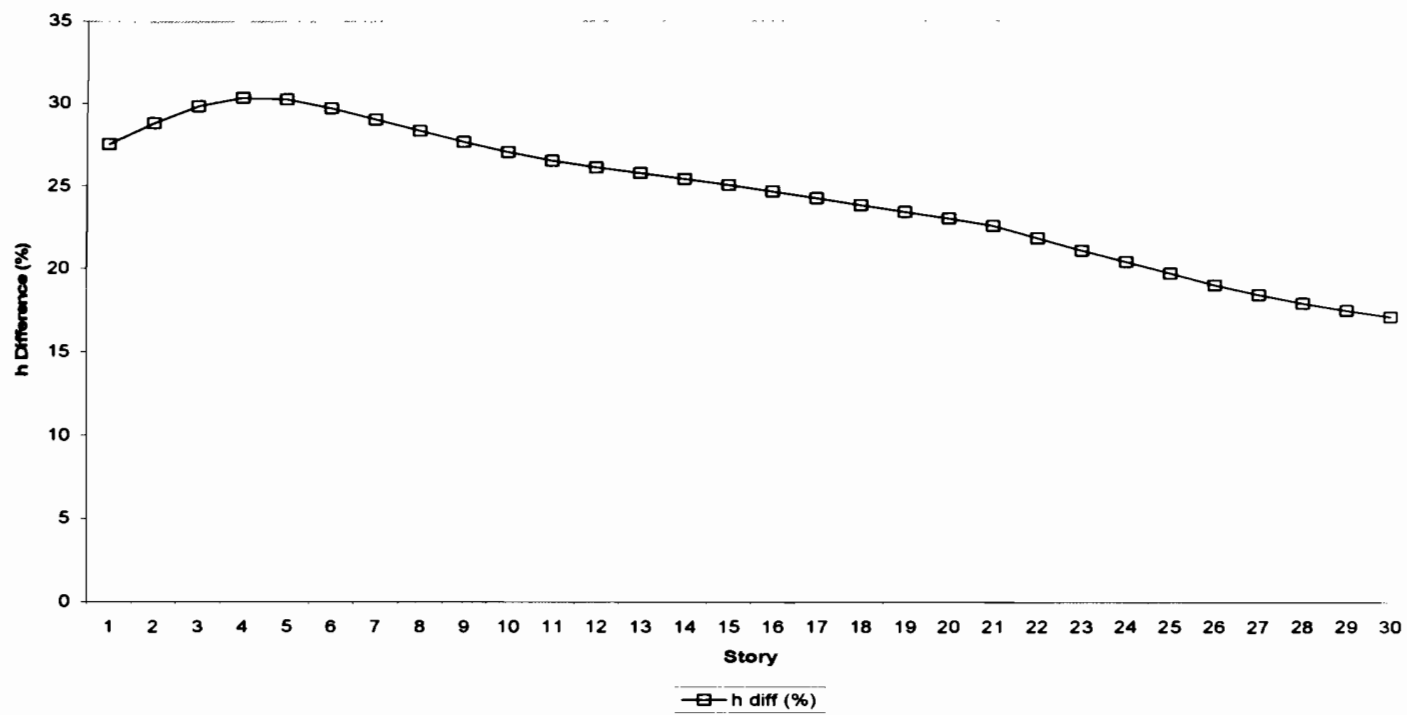

Figure 4.3.3

Percentile increase in the square cross- section dimension of a column to equate the un-cracked first order to the cracked second order lateral deflections with respect to story

2) The $24 \mathrm{ft}$ story height building:

Sway

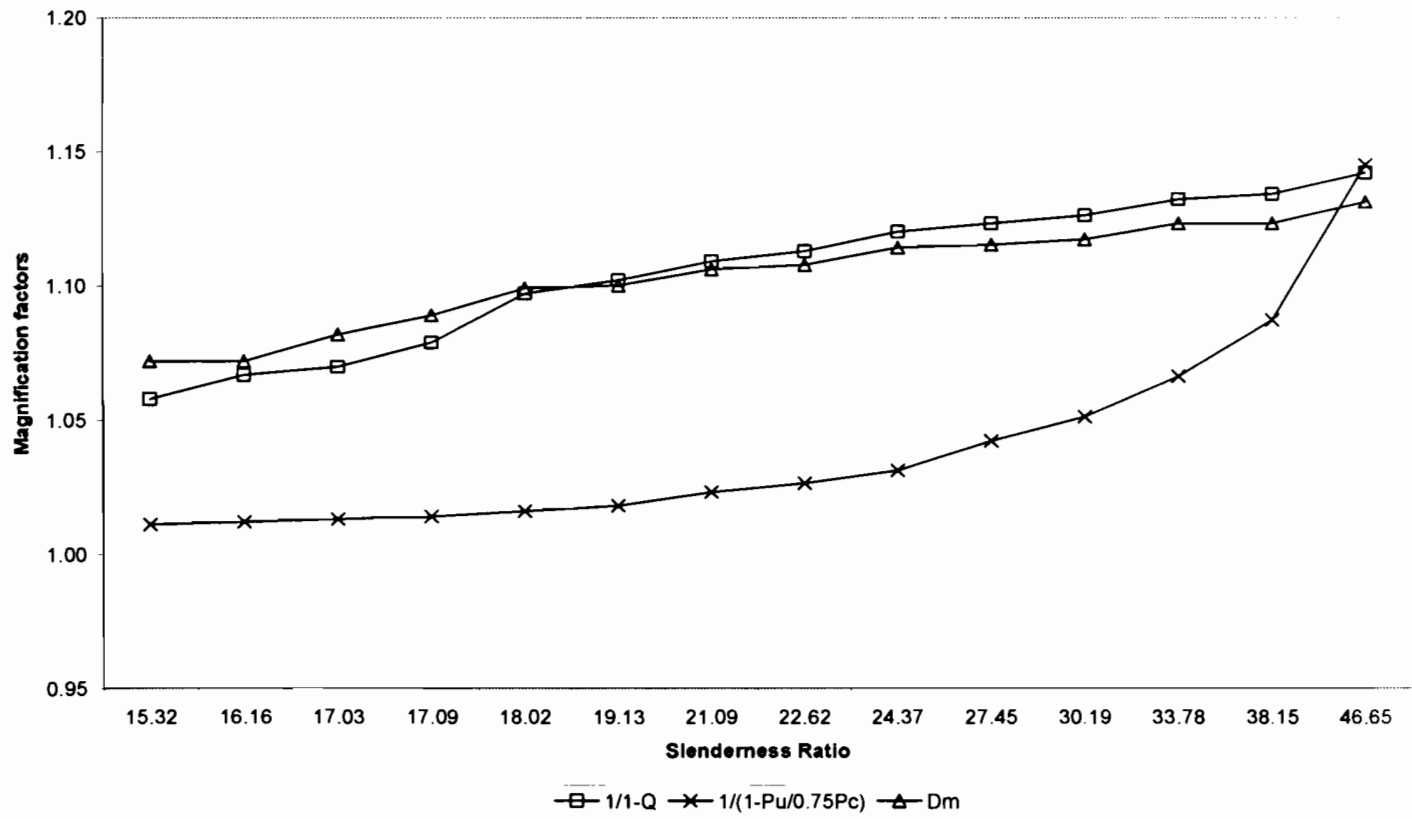

Figure 4.3.4

ACI sway magnifier factors vs. the ratio of second order to first order moments 
Braced

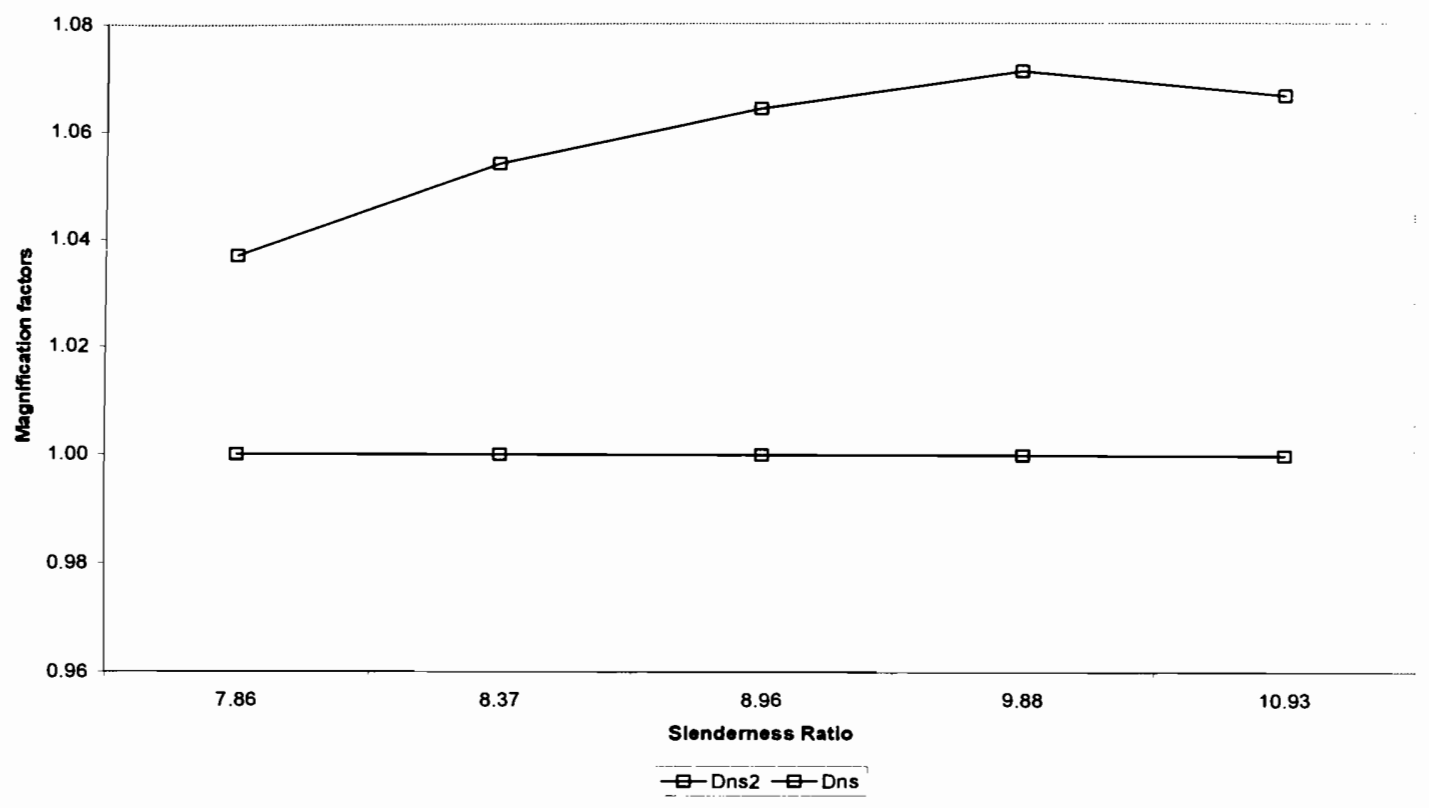

Figure 4.3.5

ACI non-sway magnifier factors vs. the ratio of second order to first order moments

\section{$h$ diff $(\%)$}

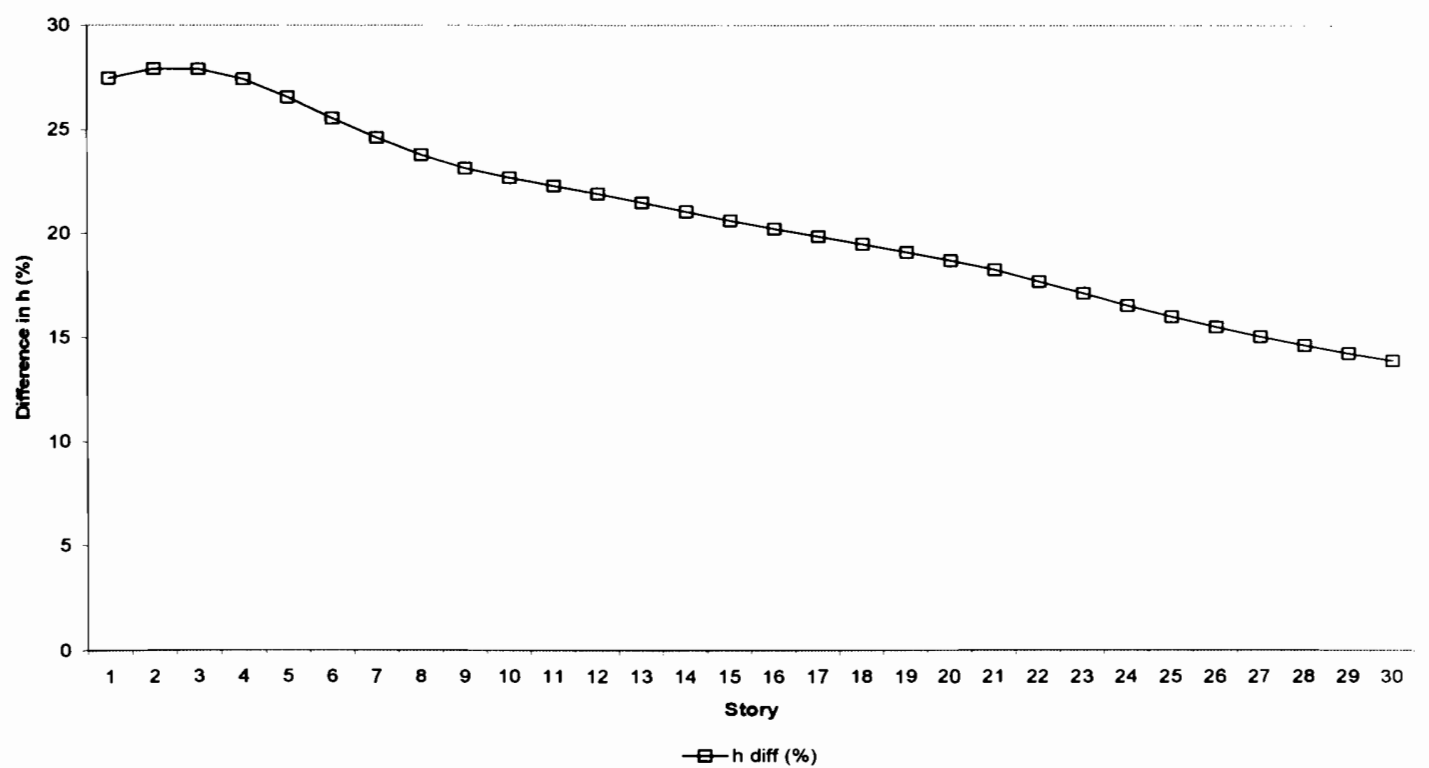

Figure 4.3.6

Percentile increase in the square cross- section dimension of a column to equate the un-cracked first order to the cracked second order lateral deflections with respect to story 
3) The $30 \mathrm{ft}$ story height building:

\section{Sway}

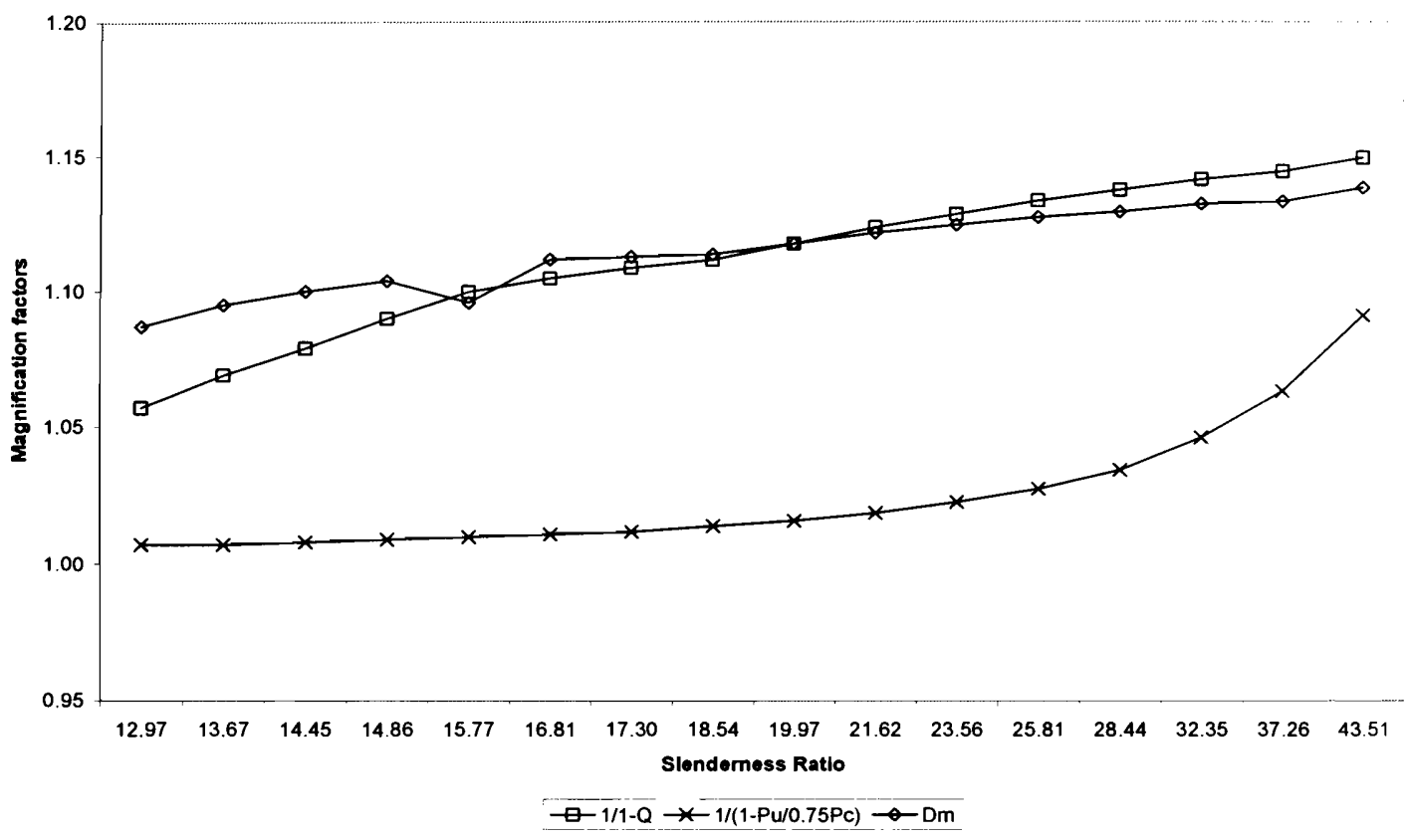

Figure 4.3.7

ACI sway magnifier factors vs. the ratio of second order to first order moments

\section{Braced}

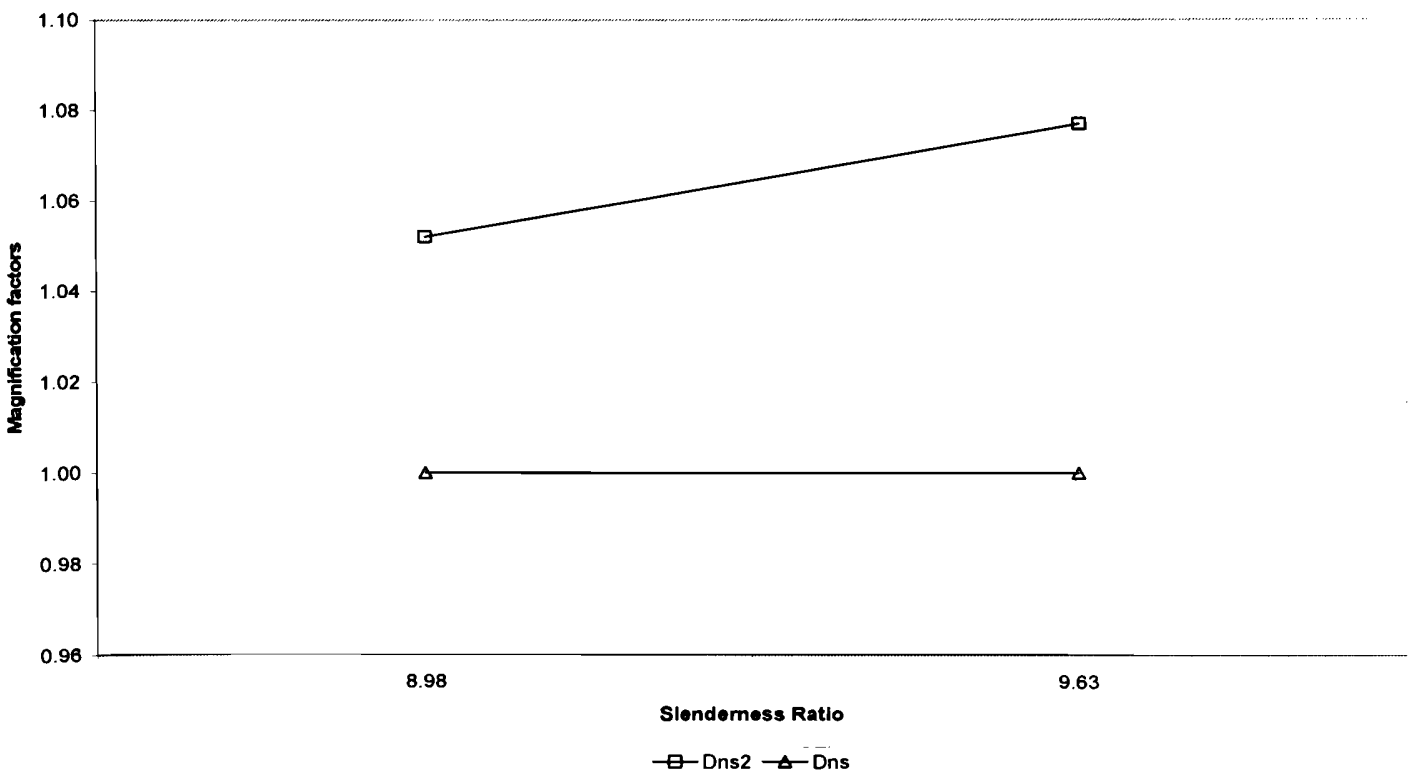

Graph 4.3.8

ACI non-sway magnifier factors vs. the ratio of second order to first order moments 
h diff (\%)

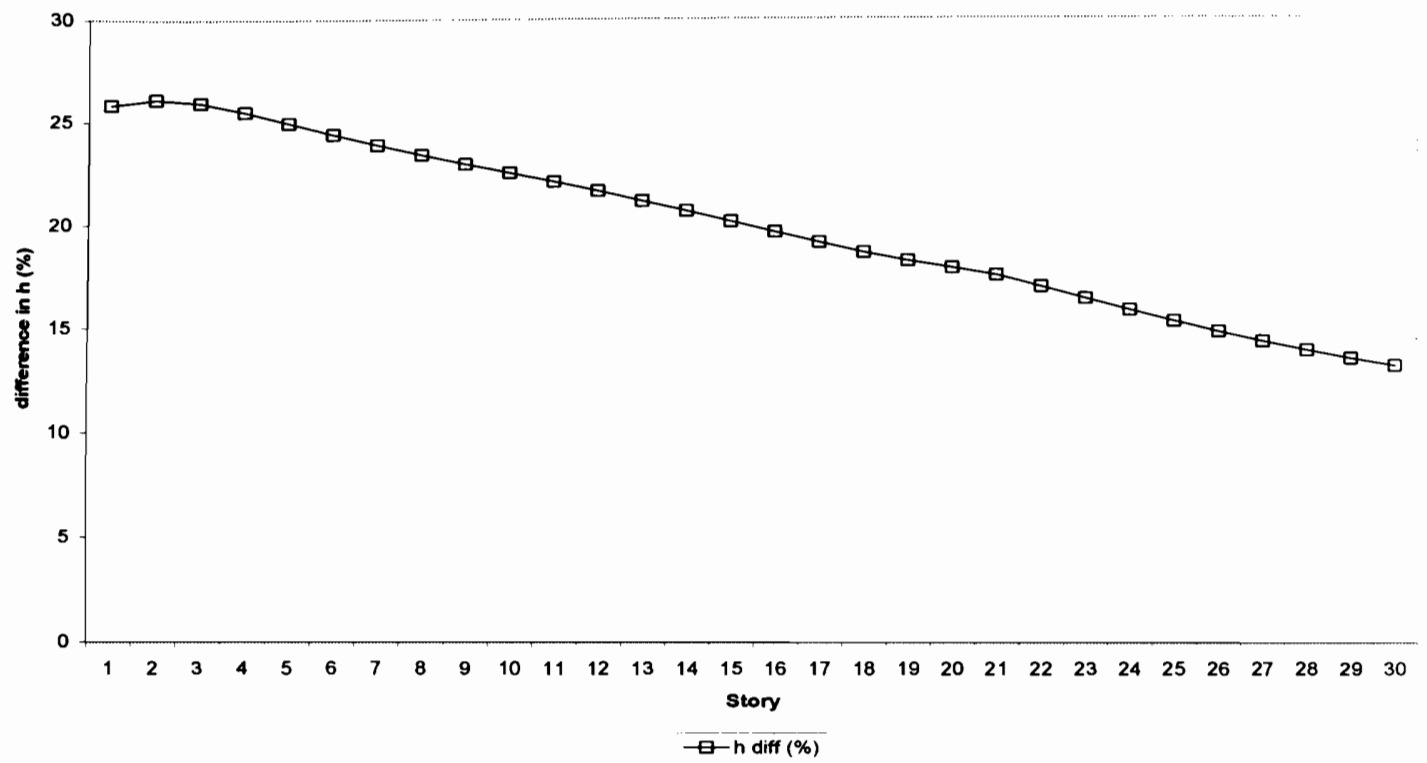

Figure 4.3.9

Percentile increase in the square cross- section dimension of a column to equate the un-cracked first order to the cracked second order lateral deflections with respect to story

4) The $36 \mathrm{ft}$ story height building:

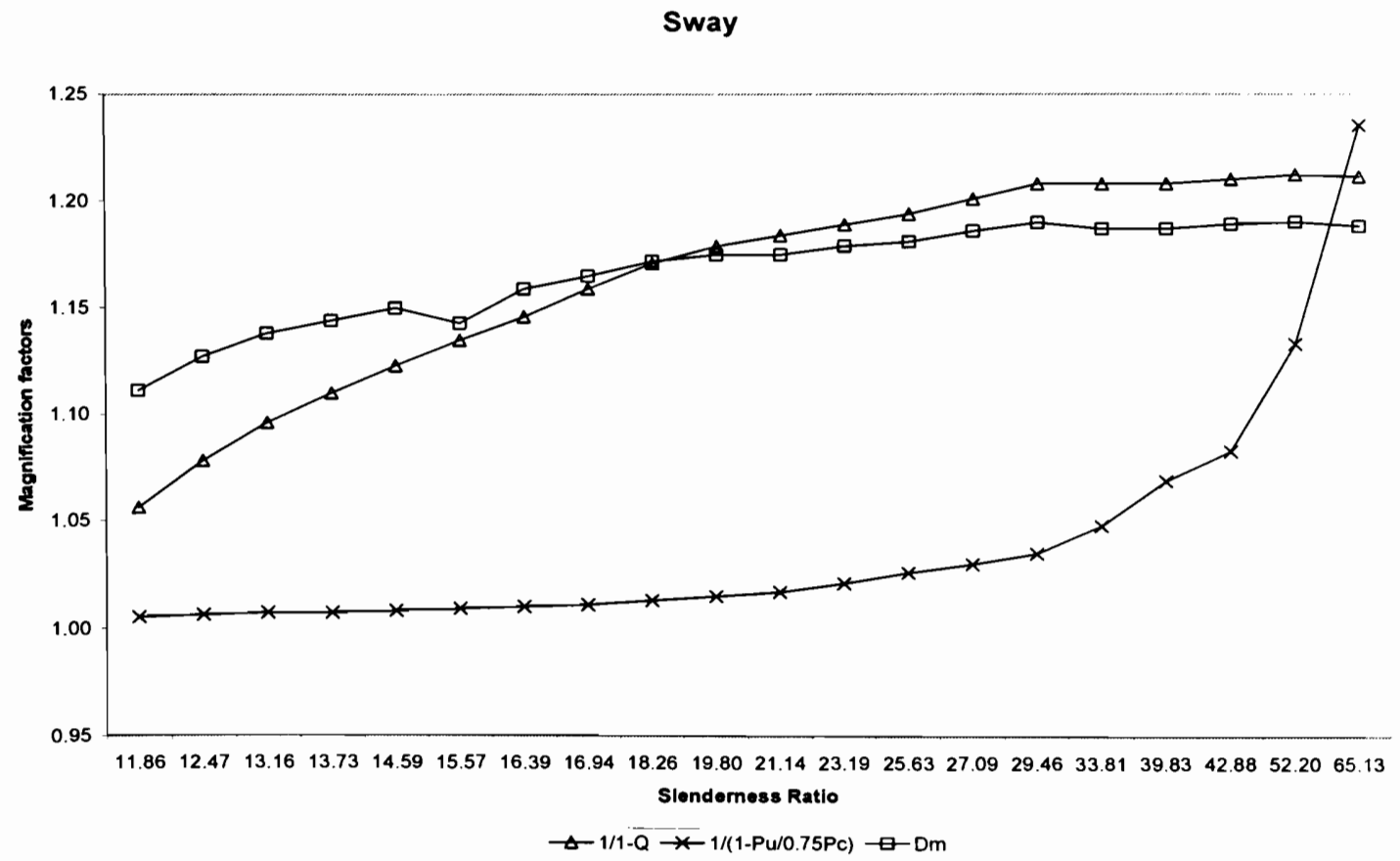

Figure 4.3.10

ACI sway magnifier factors vs. the ratio of second order to first order moments 


\section{Braced}

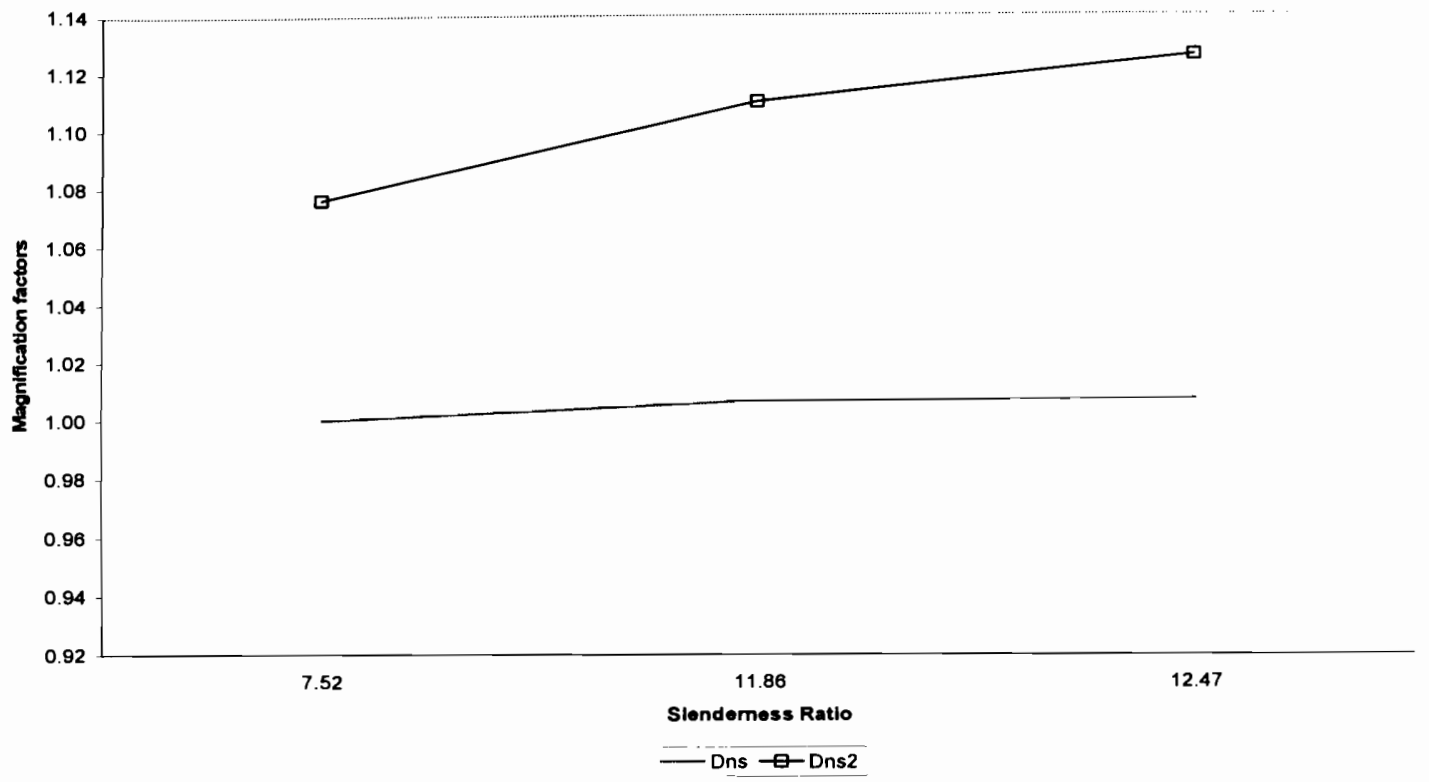

Figure 4.3.11

ACI non- sway magnifier factors vs. the ratio of second order to first order moments

h diff (\%)

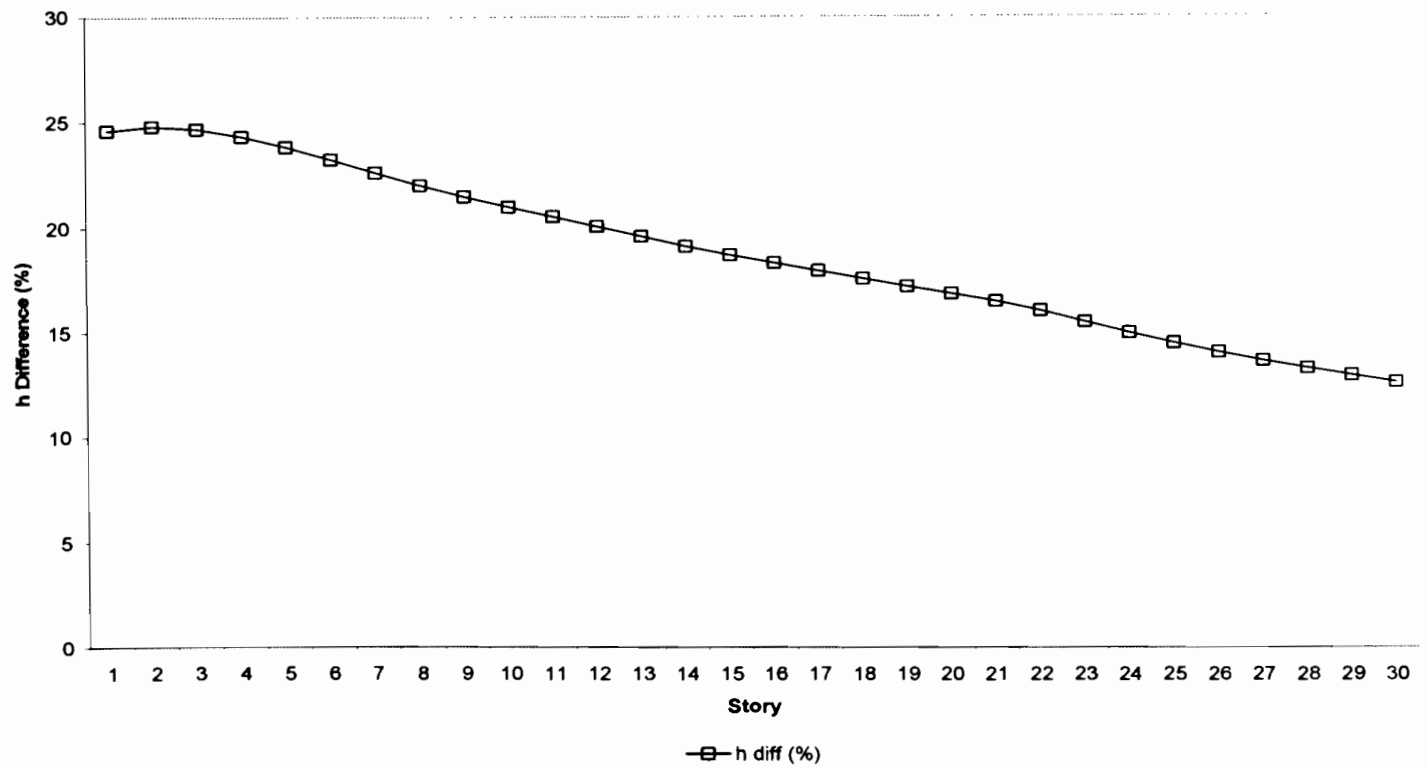

Figure 4.3.12

Percentile increase in the square cross- section dimension of a column to equate the un-cracked first order to the cracked second order lateral deflections with respect to story 
5) Difference in $\mathrm{h}(\%)$ vs. story, for all buildings:

h Diff. for all Buildings (\%)

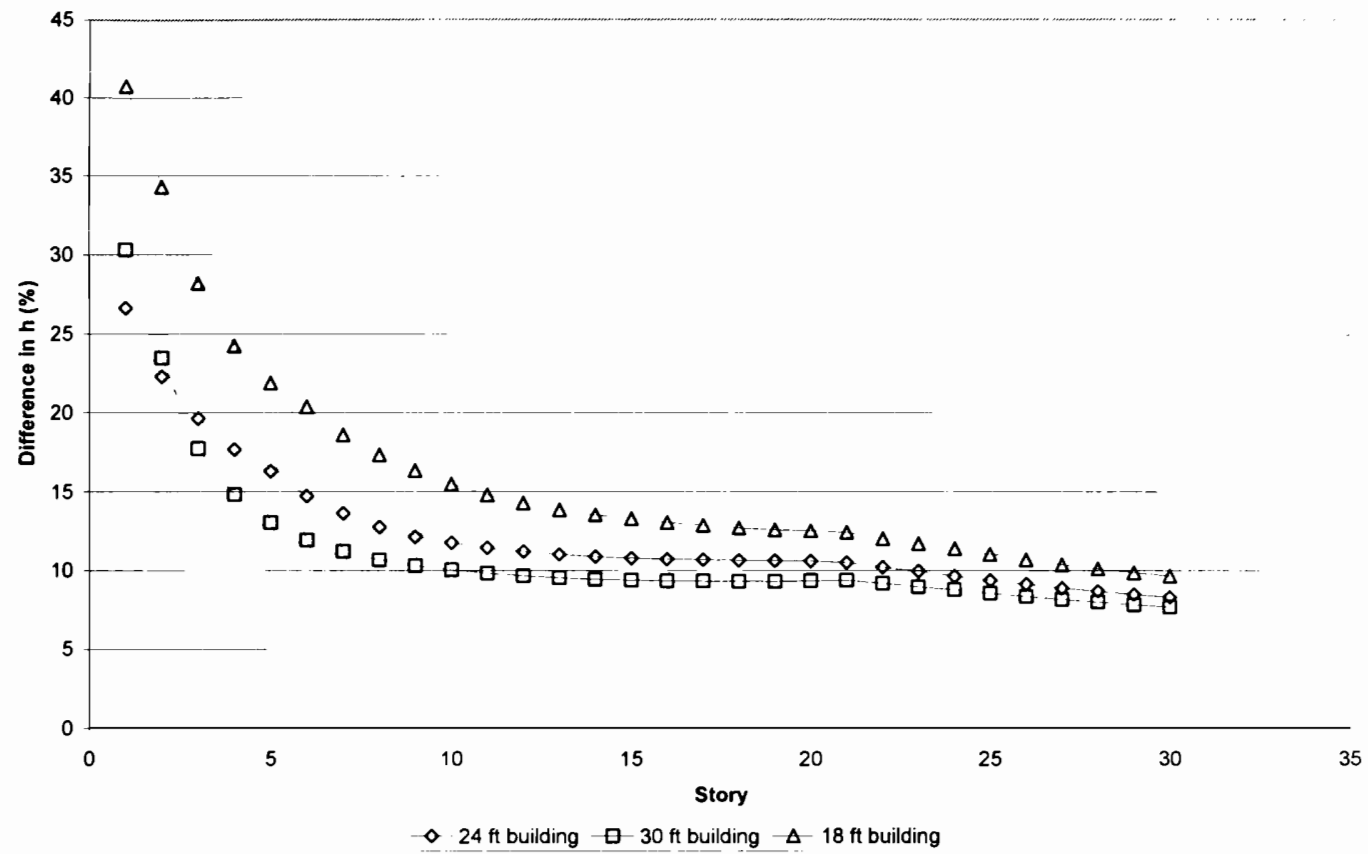

Figure 4.3.13

Percentile increase in the square cross- section dimension of a column to equate the un-cracked first order to the cracked second order lateral deflections with respect to story for all buildings 


\section{CHAPTER 5}

\section{CONCLUSIONS AND RECOMMENDATIONS}

\subsection{CONCLUSIONS:}

Conclusions are drawn with reference to the set of graphs developed for each building; the conclusions are addressed and compared to the ACI Code recommendations in three main aspects (non-sway moment magnification factor, sway moment magnification factor and cracked/un-cracked analysis results).

From the braced stories graphs set, it can be concluded that ACI code non-sway modification factor is always less than the actual difference in moments between the first order and the second order analyses. ACI permits a 5\% difference from the first order to the second order moments and according to $\mathrm{ACI}$, the second order analysis must be performed if the difference exceeds $5 \%$. That means, second order analysis must be performed for the $24 \mathrm{ft}, 30 \mathrm{ft}$ and $36 \mathrm{ft}$ story height buildings, this can be seen from figures $4.3 .11,4.3 .8$ and 4.3.5. All of the buildings except the $18 \mathrm{ft}$ story height have a moment's difference more than the ACI code 5\% limit.

The value of the ACI Code non-sway moment magnification factor is calculated using the $\mathrm{ACI}$ equation:

$$
\delta_{n s}=\frac{C_{m}}{1-\frac{P_{u}}{0.75 P_{c}}} \geq 1.0
$$


The (0.75) factor, according to ACI Code:

R10.12.3 - The $\phi$-factors used in the derigu of slen:der colunws represert two differen: sources of variabinty. First. the shifness reduction 4 factors in the nagrifier equations in the 1989 and eartier codes were intescled to account for the :anability in ile stiffiess $E I$ and the movent nuagcification analysis. Second. the variability of the streugtil of the cress section is accounted for by strength reduction $\phi$-facters of 0.70 for ied colunus and 0.75 for spiral columus. Snidies, reported an Referen:ce 30.35 indicate that the sinffness redic. tion factor $\phi_{\bar{K}}$, and the cross-secticnai strergth reduction $\phi$ factor: do not have the sane values. condrary is the as;untption in the 1959 and earlier codes. Tlese stivdies suggest the snfütess reduction factor $\phi_{\Lambda}$ for an isolared colunin should be 0.75 for boils tied and spiral colums. Tie 0.75 factors 1: Eq. (10-9) and $(10-15)$ are itiffuess reduction factors $\phi_{\Gamma}$ and replace the ofactons in these equaticals in the 1989 and earlier codes. Thus las been doue to aroid cosfusion cerveen a sinforess reduction facter $\phi_{x}$ in $\mathrm{Eq},(10-9)$ and $(10-15)$ and the cross-sectional sirengil: reduction $\phi$-incrors.

In the 1989 and earliet codes. Whe monent magriser emuation: for $\delta_{b}$ and $\delta_{s}$ unciuded a stifties: reductica facios $\phi_{K}$ is coier the uniabiaty in the stability calculation The secondrerder atalysis method is cased on the values of $E$ and $I$ arom

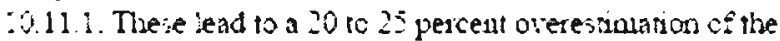
Lieral defections that corresponds to a stiffress reduction tactor $\phi_{\Delta}$ betveen 0.80 and 0.55 on ti:e PA moneuts. To addithoni $\phi$-factor is needed in the siabitity caiculation. Once the momests are estabished, selecrion of the cross sections of the columis irvolve's the stien:gh rexuction factors fon 9.3 .2$.

Based on the results of this research, we can recommend using lower values for the stiffness reduction factor for the non-sway moment magnification factor in $A C I$ equation (10-9). It will be more reasonable to use $\left(I_{c r} / I_{g}\right)$ ratio, which is actually the stiffness reduction factor, instead of using the $(0.75)$ value. That is due to the variation in the amount of cracking in the structural element in each story.

Referring to figure 4.3 .13 , this research shows that till about two-third of the building height, the extent of cracking in the structural elements is about 0.42 and after that (the rest of the upper stories) the amount of cracking starts to decrease to about 0.8 . 
In the ACI code equations, the stiffness reduction factor used for calculating the non-sway and sway moment magnification factors is the same (0.75). A conclusion can be drawn based on the results this research, the value of the stiffness reduction factor in calculating the non-way moment's modification factor should be around 0.42 till the second third of the building height. For the rest of the building a value of 0.8 can be used.

From figures 4.3.1, 4.3.4, 4.3.7 and 4.3.10, for the two values of the sway moment magnification factor $\mathrm{ACI}$ recommends to use:

$$
\begin{aligned}
& \delta_{s} M_{s}=\frac{M_{s}}{1-0} \geq M_{s} \quad(10-17) \\
& \delta_{s} M_{s}=\frac{M_{s}}{1-\frac{\Sigma P_{\text {li }}}{0.75 \Sigma P_{c}}} \geq M_{s} \quad(10-18)
\end{aligned}
$$

The actual difference in sway moments between the first order and the second order analyses results shows that $\mathrm{ACI}$ equation (10-17) is more accurate to be used in calculating the sway moment's magnifier factor.

The conclusion is a cutoff point should be set between the use of equations (1017) and (10-18). Equation (10-18) can be used for structures containing lateral forces resisting systems (shear walls, braces, etc) and the value of the stiffness reduction factor in equation (10-18) should vary with respect to the story in interest, as suggested earlier.

Equation (10-17) can be used for braced or sway structures, from the graphs it can be seen that for a slenderness ratio greater than 22 equation (10-17) is the most accurate in calculating the sway moment magnification factor.

In the second part of this investigation, using cracked sections in the second order analysis, graphs were drawn to find out how much the sections should be increased to get 
the same lateral deflection of the first order un-cracked analysis. The percentage increase was shown for each story for the three buildings. The maximum increase is about $30 \%$ and the minimum is around $15 \%$.

It can be concluded that the critical stories in a building are till the second third of the building height. In other words, the first two thirds of a building height should be analyzed using the cracked moment of inertia (modified stiffnesses), since they are most critically affected by lateral deflections of the building. The remaining stories could be analyzed using un-cracked sections (full stiffnesses). 


\subsection{RECOMMENDATIONS FOR FUTURE STUDY:}

During the work on this investigation, a set of assumptions were made to simplify the procedure of analysis and design. These simplifications can be investigated further as follows:

First, the building configuration used in this research, one bay by one bay. More complicated configurations can be investigated to add more variation in the results.

Second, all the columns in each building have square cross sections. Other sections can be investigated for columns; this may lead to more specific results regarding other types of cross sections.

Third, no bracing systems (shearwalls, braces...etc) were considered in the buildings. Further investigations can be done for buildings containing lateral loads resisting systems.

Fourth, only 30 story buildings were investigated. Different number of stories can be studied to understand the behavior of buildings having more or less number of stories. 


\section{REFERENCES:}

1) ACI Committee 318, 2002, "Building Code Requirements for Structural Concrete (ACI 318-02) and Commentary (318R-02)," American Concrete Institute. Farmington Hills, Mich., 443pp.

2) American Institute of steel Construction, 1993, "Load Resistance Factor Design Specification for Structural Steel Buildings," Chicago, Ill.

3) Columns as a Part of a Rectangular Frame," ACI Journal, Proceedings V. 61, No. 5, May pp. 563-587.

4) Ferguson, P. M., and Breen, J. E., 1996, "Investigation of the Long Column in a Frame Subject to Lateral Loads," Reinforced Concrete Columns, SP-13. American Concrete Institute, Farmington Hills, Mich. Pp. 75-119. 
APPENDIX 


\begin{tabular}{|c|c|c|c|c|c|}
\hline Story & Load & Loc (in) & $\mathrm{P}$ (kips) & V2 (kips) & M3 (kips-in) \\
\hline STORY30 & DEAD & 0.000 & -66.326 & -0.669 & -95.505 \\
\hline STORY30 & DEAD & 119.000 & -62.979 & -0.669 & -15.887 \\
\hline STORY30 & DEAD & 238.000 & -59.633 & -0.669 & 63.731 \\
\hline STORY30 & LIVE & 0.000 & -11.520 & -0.180 & -25.657 \\
\hline STORY30 & LIVE & 119.000 & -11.520 & -0.180 & -4.268 \\
\hline STORY30 & LIVE & 238.000 & -11.520 & -0.180 & 17.121 \\
\hline STORY30 & WIND & 0.000 & 5.850 & 5.820 & 830.666 \\
\hline STORY30 & WIND & 119.000 & 5.850 & 5.820 & 138.105 \\
\hline STORY30 & WIND & 238.000 & 5.850 & 5.820 & -554.456 \\
\hline STORY29 & DEAD & 0.000 & -132.652 & -0.631 & -89.811 \\
\hline STORY29 & DEAD & 119.000 & -129.305 & -0.631 & -14.750 \\
\hline STORY29 & DEAD & 238.000 & -125.959 & -0.631 & 60.311 \\
\hline STORY29 & LIVE & 0.000 & -23.040 & -0.169 & -24.128 \\
\hline STORY29 & LIVE & 119.000 & -23.040 & -0.169 & -3.963 \\
\hline STORY29 & LIVE & 238.000 & -23.040 & -0.169 & 16.203 \\
\hline STORY29 & WIND & 0.000 & 29.032 & 17.398 & 2499.415 \\
\hline STORY29 & WIND & 119.000 & 29.032 & 17.398 & 429.083 \\
\hline STORY29 & WIND & 238.000 & 29.032 & 17.398 & -1641.248 \\
\hline STORY28 & DEAD & 0.000 & -200.878 & -1.423 & -202.660 \\
\hline STORY28 & DEAD & 119.000 & -196.746 & -1.423 & -33.371 \\
\hline STORY28 & DEAD & 238.000 & -192.614 & -1.423 & 135.919 \\
\hline STORY28 & LIVE & 0.000 & -34.560 & -0.382 & -54.445 \\
\hline STORY28 & LIVE & 119.000 & -34.560 & -0.382 & -8.965 \\
\hline STORY28 & LIVE & 238.000 & -34.560 & -0.382 & 36.515 \\
\hline STORY28 & WIND & 0.000 & 75.297 & 28.874 & 4148.424 \\
\hline STORY28 & WIND & 119.000 & 75.297 & 28.874 & 712.437 \\
\hline STORY28 & WIND & 238.000 & 75.297 & 28.874 & -2723.551 \\
\hline STORY27 & DEAD & 0.000 & -269.104 & -1.309 & -185.248 \\
\hline STORY27 & DEAD & 119.000 & -264.972 & -1.309 & -29.423 \\
\hline STORY27 & DEAD & 238.000 & -260.840 & -1.309 & 126.401 \\
\hline STORY27 & LIVE & 0.000 & -46.080 & -0.352 & -49.767 \\
\hline STORY27 & LIVE & 119.000 & -46.080 & -0.352 & -7.905 \\
\hline STORY27 & LIVE & 238.000 & -46.080 & -0.352 & 33.958 \\
\hline STORY27 & WIND & 0.000 & 144.272 & 40.252 & 5802.874 \\
\hline STORY27 & WIND & 119.000 & 144.272 & 40.252 & 1012.945 \\
\hline STORY27 & WIND & 238.000 & 144.272 & 40.252 & -3776.984 \\
\hline STORY26 & DEAD & 0.000 & -341.729 & -2.436 & -344.711 \\
\hline STORY26 & DEAD & 119.000 & -335.779 & -2.436 & -54.868 \\
\hline STORY26 & DEAD & 238.000 & -329.830 & -2.436 & 234.976 \\
\hline STORY26 & LIVE & 0.000 & -57.600 & -0.654 & -92.607 \\
\hline STORY26 & LIVE & 119.000 & -57.600 & -0.654 & -14.740 \\
\hline STORY26 & LIVE & 238.000 & -57.600 & -0.654 & 63.126 \\
\hline STORY26 & WIND & 0.000 & 235.870 & 51.519 & 7442.750 \\
\hline STORY26 & WIND & 119.000 & 235.870 & 51.519 & 1312.001 \\
\hline STORY26 & WIND & 238.000 & 235.870 & 51.519 & -4818.747 \\
\hline STORY25 & DEAD & 0.000 & -414.355 & $-2 . \overline{245}$ & -320.026 \\
\hline
\end{tabular}




\begin{tabular}{|c|c|c|c|c|c|}
\hline STORY25 & DEAD & 119.000 & -408.405 & -2.245 & -52.836 \\
\hline STORY25 & DEAD & 238.000 & -402.455 & -2.245 & 214.354 \\
\hline STORY25 & LIVE & 0.000 & -69.120 & -0.603 & -85.975 \\
\hline STORY25 & LIVE & 119.000 & -69.120 & -0.603 & -14.194 \\
\hline STORY25 & LIVE & 238.000 & -69.120 & -0.603 & 57.586 \\
\hline STORY25 & WIND & 0.000 & 349.743 & 62.686 & 9089.242 \\
\hline STORY25 & WIND & 119.000 & 349.743 & 62.686 & 1629.576 \\
\hline STORY25 & WIND & 238.000 & 349.743 & 62.686 & -5830.089 \\
\hline STORY24 & DEAD & 0.000 & -489.480 & -2.901 & -413.326 \\
\hline STORY24 & DEAD & 119.000 & -482.497 & -2.901 & -68.145 \\
\hline STORY24 & DEAD & 238.000 & -475.515 & -2.901 & 277.036 \\
\hline STORY24 & LIVE & 0.000 & -80.640 & -0.779 & -111.040 \\
\hline STORY24 & LIVE & 119.000 & -80.640 & -0.779 & -18.307 \\
\hline STORY24 & LIVE & 238.000 & -80.640 & -0.779 & 74.426 \\
\hline STORY24 & WIND & 0.000 & 485.710 & 73.738 & 10734.270 \\
\hline STORY24 & WIND & 119.000 & 485.710 & 73.738 & 1959.476 \\
\hline STORY24 & WIND & 238.000 & 485.710 & 73.738 & -6815.323 \\
\hline STORY23 & DEAD & 0.000 & -564.605 & -2.728 & -388.667 \\
\hline STORY23 & DEAD & 119.000 & -557.623 & -2.728 & -64.068 \\
\hline STORY23 & DEAD & 238.000 & -550.640 & -2.728 & 260.531 \\
\hline STORY23 & LIVE & 0.000 & -92.160 & -0.733 & -104.416 \\
\hline STORY23 & LIVE & 119.000 & -92.160 & -0.733 & -17.212 \\
\hline STORY23 & LIVE & 238.000 & -92.160 & -0.733 & 69.992 \\
\hline STORY23 & WIND & 0.000 & 643.585 & 84.683 & 12373.550 \\
\hline STORY23 & WIND & 119.000 & 643.585 & 84.683 & 2296.248 \\
\hline STORY23 & WIND & 238.000 & 643.585 & 84.683 & -7781.054 \\
\hline STORY22 & DEAD & 0.000 & -642.431 & -3.286 & -460.528 \\
\hline STORY22 & DEAD & 119.000 & -634.333 & -3.286 & -69.484 \\
\hline STORY22 & DEAD & 238.000 & -626.234 & -3.286 & 321.560 \\
\hline STORY22 & LIVE & 0.000 & -103.680 & -0.883 & -123.721 \\
\hline STORY22 & LIVE & 119.000 & -103.680 & -0.883 & -18.667 \\
\hline STORY22 & LIVE & 238.000 & -103.680 & -0.883 & 86.387 \\
\hline STORY22 & WIND & 0.000 & 822.951 & 95.502 & 14029.120 \\
\hline STORY22 & WIND & 119.000 & 822.951 & 95.502 & 2664.336 \\
\hline STORY22 & WIND & 238.000 & 822.951 & 95.502 & -8700.447 \\
\hline STORY21 & DEAD & 0.000 & -723.156 & -3.852 & -548.468 \\
\hline STORY21 & DEAD & 119.000 & -713.859 & -3.852 & -90.122 \\
\hline STORY21 & DEAD & 238.000 & -704.563 & -3.852 & 368.223 \\
\hline STORY21 & LIVE & 0.000 & -115.200 & -1.035 & -147.346 \\
\hline STORY21 & LIVE & 119.000 & -115.200 & -1.035 & -24.211 \\
\hline STORY21 & LIVE & 238.000 & -115.200 & -1.035 & 98.923 \\
\hline STORY21 & WIND & 0.000 & 1023.563 & 106.197 & 15697.870 \\
\hline STORY21 & WIND & 119.000 & 1023.563 & 106.197 & 3060.485 \\
\hline STORY21 & WIND & 238.000 & 1023.563 & 106.197 & -9576.903 \\
\hline STORY20 & DEAD & 0.000 & -803.881 & -3.626 & -517.070 \\
\hline STORY20 & DEAD & 119.000 & -794.584 & -3.626 & -85.621 \\
\hline STORY20 & DEAD & 238.000 & -785.288 & -3.626 & 345.828 \\
\hline
\end{tabular}




\begin{tabular}{|c|c|c|c|c|c|}
\hline STORY20 & LIVE & 0.000 & -126.720 & -0.974 & -138.911 \\
\hline STORY20 & LIVE & 119.000 & -126.720 & -0.974 & -23.002 \\
\hline STORY20 & LIVE & 238.000 & -126.720 & -0.974 & 92.907 \\
\hline STORY20 & WIND & 0.000 & 1245.454 & 116.781 & 17342.880 \\
\hline STORY20 & WIND & 119.000 & 1245.454 & 116.781 & 3445.924 \\
\hline STORY20 & WIND & 238.000 & 1245.454 & 116.781 & -10451.030 \\
\hline STORY19 & DEAD & 0.000 & -887.705 & -3.996 & -548.259 \\
\hline STORY19 & DEAD & 119.000 & -877.128 & -3.996 & -72.712 \\
\hline STORY19 & DEAD & 238.000 & -866.551 & -3.996 & 402.835 \\
\hline STORY19 & LIVE & 0.000 & -138.240 & -1.074 & -147.290 \\
\hline STORY19 & LIVE & 119.000 & -138.240 & -1.074 & -19.534 \\
\hline STORY19 & LIVE & 238.000 & -138.240 & -1.074 & 108.222 \\
\hline STORY19 & WIND & 0.000 & 1487.728 & 127.216 & 19047.290 \\
\hline STORY19 & WIND & 119.000 & 1487.728 & 127.216 & 3908.557 \\
\hline STORY19 & WIND & 238.000 & 1487.728 & 127.216 & -11230.180 \\
\hline STORY18 & DEAD & 0.000 & -978.330 & -5.132 & -721.730 \\
\hline STORY18 & DEAD & 119.000 & -964.943 & -5.132 & -111.070 \\
\hline STORY18 & DEAD & 238.000 & -951.557 & -5.132 & 499.590 \\
\hline STORY18 & LIVE & 0.000 & -149.760 & -1.379 & -193.893 \\
\hline STORY18 & LIVE & 119.000 & -149.760 & -1.379 & -29.839 \\
\hline STORY18 & LIVE & 238.000 & -149.760 & -1.379 & 134.215 \\
\hline STORY18 & WIND & 0.000 & 1749.705 & 137.470 & 20847.700 \\
\hline STORY18 & WIND & 119.000 & 1749.705 & 137.470 & 4488.725 \\
\hline STORY18 & WIND & 238.000 & 1749.705 & 137.470 & -11870.250 \\
\hline STORY17 & DEAD & 0.000 & -1068.954 & -4.428 & -619.603 \\
\hline STORY17 & DEAD & 119.000 & -1055.568 & -4.428 & -92.645 \\
\hline STORY17 & DEAD & 238.000 & -1042.181 & -4.428 & 434.314 \\
\hline STORY17 & LIVE & 0.000 & -161.280 & -1.190 & -166.457 \\
\hline STORY17 & LIVE & 119.000 & -161.280 & -1.190 & -24.889 \\
\hline STORY17 & LIVE & 238.000 & -161.280 & -1.190 & 116.679 \\
\hline STORY17 & WIND & 0.000 & 2032.553 & 147.661 & 22558.210 \\
\hline STORY17 & WIND & 119.000 & 2032.553 & 147.661 & 4986.564 \\
\hline STORY17 & WIND & 238.000 & 2032.553 & 147.661 & -12585.090 \\
\hline STORY16 & DEAD & 0.000 & -1167.178 & -5.608 & -780.697 \\
\hline STORY16 & DEAD & 119.000 & -1150.651 & -5.608 & -113.331 \\
\hline STORY16 & DEAD & 238.000 & -1134.125 & -5.608 & 554.036 \\
\hline STORY16 & LIVE & 0.000 & -172.800 & -1.507 & -209.735 \\
\hline STORY16 & LIVE & 119.000 & -172.800 & -1.507 & -30.446 \\
\hline STORY16 & LIVE & 238.000 & -172.800 & -1.507 & 148.842 \\
\hline STORY 16 & WIND & 0.000 & 2333.716 & 157.581 & 24459.700 \\
\hline STORY16 & WIND & 119.000 & 2333.716 & 157.581 & 5707.589 \\
\hline STORY16 & WIND & 238.000 & 2333.716 & 157.581 & -13044.520 \\
\hline STORY15 & DEAD & 0.000 & -1265.402 & -5.259 & -766.487 \\
\hline STORY 15 & DEAD & 119.000 & -1248.875 & -5.259 & -140.662 \\
\hline STORY15 & DEAD & 238.000 & -1232.349 & -5.259 & 485.164 \\
\hline STORY15 & LIVE & 0.000 & -184.320 & -1.413 & -205.917 \\
\hline STORY15 & LIVE & 119.000 & -184.320 & -1.413 & -37.789 \\
\hline
\end{tabular}




\begin{tabular}{|c|c|c|c|c|c|}
\hline STORY 15 & LIVE & 238.000 & -184.320 & -1.413 & 130.340 \\
\hline STORY15 & WIND & 0.000 & 2655.446 & 167.443 & 26206.720 \\
\hline STORY15 & WIND & 119.000 & 2655.446 & 167.443 & 6280.972 \\
\hline STORY15 & WIND & 238.000 & 2655.446 & 167.443 & -13644.780 \\
\hline STORY14 & DEAD & 0.000 & -1363.626 & -4.941 & -686.130 \\
\hline STORY14 & DEAD & 119.000 & -1347.099 & -4.941 & -98.196 \\
\hline STORY14 & DEAD & 238.000 & -1330.572 & -4.941 & 489.737 \\
\hline STORY14 & LIVE & 0.000 & -195.840 & -1.327 & -184.334 \\
\hline STORY 14 & LIVE & 119.000 & -195.840 & -1.327 & -26.383 \\
\hline STORY14 & LIVE & 238.000 & -195.840 & -1.327 & 131.569 \\
\hline STORY14 & WIND & 0.000 & 2996.220 & 177.148 & 27973.630 \\
\hline STORY14 & WIND & 119.000 & 2996.220 & 177.148 & 6893.083 \\
\hline STORY14 & WIND & 238.000 & 2996.220 & 177.148 & -14187.460 \\
\hline STORY13 & DEAD & 0.000 & -1470.249 & -6.016 & -845.089 \\
\hline STORY13 & DEAD & 119.000 & -1450.252 & -6.016 & -129.163 \\
\hline STORY13 & DEAD & 238.000 & -1430.255 & -6.016 & 586.763 \\
\hline STORY13 & LIVE & 0.000 & -207.360 & -1.616 & -227.003 \\
\hline STORY13 & LIVE & 119.000 & -207.360 & -1.616 & -34.685 \\
\hline STORY13 & LIVE & 238.000 & -207.360 & -1.616 & 157.632 \\
\hline STORY13 & WIND & 0.000 & 3352.998 & 186.459 & 30058.060 \\
\hline STORY13 & WIND & 119.000 & 3352.998 & 186.459 & 7869.419 \\
\hline STORY13 & WIND & 238.000 & 3352.998 & 186.459 & -14319.220 \\
\hline STORY12 & DEAD & 0.000 & -1576.872 & -5.196 & -727.393 \\
\hline STORY12 & DEAD & 119.000 & -1556.875 & -5.196 & -109.104 \\
\hline STORY12 & DEAD & 238.000 & -1536.878 & -5.196 & 509.185 \\
\hline STORY12 & LIVE & 0.000 & -218.880 & -1.397 & -195.582 \\
\hline STORY12 & LIVE & 119.000 & -218.880 & -1.397 & -29.387 \\
\hline STORY12 & LIVE & 238.000 & -218.880 & -1.397 & 136.807 \\
\hline STORY12 & WIND & 0.000 & 3729.166 & 195.801 & 31974.820 \\
\hline STORY12 & WIND & 119.000 & 3729.166 & 195.801 & 8674.538 \\
\hline STORY12 & WIND & 238.000 & 3729.166 & 195.801 & -14625.740 \\
\hline STORY11 & DEAD & 0.000 & -1692.694 & -6.279 & -882.220 \\
\hline STORY11 & DEAD & 119.000 & -1668.896 & -6.279 & -134.984 \\
\hline STORY11 & DEAD & 238.000 & -1645.098 & -6.279 & 612.252 \\
\hline STORY11 & LIVE & 0.000 & -230.400 & -1.682 & -236.002 \\
\hline STORY11 & LIVE & 119.000 & -230.400 & -1.682 & -35.786 \\
\hline STORY11 & LIVE & 238.000 & -230.400 & -1.682 & 164.431 \\
\hline STORY11 & WIND & 0.000 & 4119.290 & 205.002 & 34463.370 \\
\hline STORY11 & WIND & 119.000 & 4119.290 & 205.002 & 10068.100 \\
\hline STORY11 & WIND & 238.000 & 4119.290 & 205.002 & -14327.170 \\
\hline STORY10 & DEAD & 0.000 & -1808.513 & -5.395 & -752.966 \\
\hline STORY10 & DEAD & 119.000 & -1784.714 & -5.395 & -111.004 \\
\hline STORY10 & DEAD & 238.000 & -1760.916 & -5.395 & 530.959 \\
\hline STORY10 & LIVE & 0.000 & -241.918 & -1.472 & -207.288 \\
\hline STORY10 & LIVE & 119.000 & -241.918 & -1.472 & -32.147 \\
\hline STORY10 & LIVE & 238.000 & -241.918 & -1.472 & 142.994 \\
\hline STORY10 & WIND & 0.000 & 4522.587 & 211.923 & 36936.360 \\
\hline
\end{tabular}




\begin{tabular}{|c|c|c|c|c|c|}
\hline STORY10 & WIND & 119.000 & 4522.587 & 211.923 & 11717.490 \\
\hline STORY 10 & WIND & 238.000 & 4522.587 & 211.923 & -13501.370 \\
\hline STORY9 & DEAD & 0.000 & -1934.313 & -6.674 & -959.145 \\
\hline STORY9 & DEAD & 119.000 & -1906.382 & -6.674 & -164.949 \\
\hline STORY9 & DEAD & 238.000 & -1878.452 & -6.674 & 629.247 \\
\hline STORY9 & LIVE & 0.000 & -253.431 & -1.669 & -229.258 \\
\hline STORY9 & LIVE & 119.000 & -253.431 & -1.669 & -30.662 \\
\hline STORY9 & LIVE & 238.000 & -253.431 & -1.669 & 167.935 \\
\hline STORY9 & WIND & 0.000 & 4922.562 & 240.513 & 49061.390 \\
\hline STORY9 & WIND & 119.000 & 4922.562 & 240.513 & 20440.340 \\
\hline STORY9 & WIND & 238.000 & 4922.562 & 240.513 & -8180.706 \\
\hline STORY8 & DEAD & 0.000 & -2067.276 & -6.476 & -924.026 \\
\hline STORY8 & DEAD & 114.000 & -2040.519 & -6.476 & -185.776 \\
\hline STORY 8 & DEAD & 228.000 & -2013.763 & -6.476 & 552.473 \\
\hline STORY8 & LIVE & 0.000 & -264.970 & -1.452 & -208.856 \\
\hline STORY8 & LIVE & 114.000 & -264.970 & -1.452 & -43.273 \\
\hline STORY8 & LIVE & 228.000 & -264.970 & -1.452 & 122.310 \\
\hline STORY 8 & WIND & 0.000 & 5458.718 & 249.547 & 45692.610 \\
\hline STORY 8 & WIND & 114.000 & 5458.718 & 249.547 & 17244.210 \\
\hline STORY 8 & WIND & 228.000 & 5458.718 & 249.547 & -11204.180 \\
\hline STORY7 & DEAD & 0.000 & -2205.498 & -6.597 & -913.200 \\
\hline STORY7 & DEAD & 114.000 & -2176.644 & -6.597 & -161.105 \\
\hline STORY7 & DEAD & 228.000 & -2147.790 & -6.597 & 590.990 \\
\hline STORY7 & LIVE & 0.000 & -276.482 & -1.506 & -208.095 \\
\hline STORY7 & LIVE & 114.000 & -276.482 & -1.506 & -36.431 \\
\hline STORY7 & LIVE & 228.000 & -276.482 & -1.506 & 135.233 \\
\hline STORY7 & WIND & 0.000 & 5943.690 & 236.756 & 44302.780 \\
\hline STORY7 & WIND & 114.000 & 5943.690 & 236.756 & 17312.610 \\
\hline STORY7 & WIND & 228.000 & 5943.690 & 236.756 & -9677.565 \\
\hline STORY6 & DEAD & 0.000 & -2354.934 & -7.116 & -991.090 \\
\hline STORY6 & DEAD & 114.000 & -2321.646 & -7.116 & -179.839 \\
\hline STORY6 & DEAD & 228.000 & -2288.359 & -7.116 & 631.412 \\
\hline STORY6 & LIVE & 0.000 & -288.001 & -1.624 & -226.573 \\
\hline STORY6 & LIVE & 114.000 & -288.001 & -1.624 & -41.461 \\
\hline STORY6 & LIVE & 228.000 & -288.001 & -1.624 & 143.650 \\
\hline STORY6 & WIND & 0.000 & 6417.734 & 248.415 & 47087.490 \\
\hline STORY6 & WIND & 114.000 & 6417.734 & 248.415 & 18768.180 \\
\hline STORY6 & WIND & 228.000 & 64.17 .734 & 248.415 & -9551.133 \\
\hline STORY5 & DEAD & 0.000 & -2510.273 & -6.939 & -975.553 \\
\hline STORY5 & DEAD & 114.000 & -2474.650 & -6.939 & -184.521 \\
\hline STORY5 & DEAD & 228.000 & -2439.028 & -6.939 & 606.511 \\
\hline STORY5 & LIVE & 0.000 & -299.520 & -1.559 & -217.291 \\
\hline STORY5 & LIVE & 114.000 & -299.520 & -1.559 & -39.570 \\
\hline STORY5 & LIVE & 228.000 & -299.520 & -1.559 & 138.151 \\
\hline STORY5 & WIND & 0.000 & 6920.076 & 256.248 & 48195.830 \\
\hline STORY5 & WIND & 114.000 & 6920.076 & 256.248 & 18983.520 \\
\hline STORY 5 & WIND & 228.000 & 6920.076 & 256.248 & -10228.790 \\
\hline
\end{tabular}




\begin{tabular}{|c|c|c|c|c|c|}
\hline STORY4 & DEAD & 0.000 & -2678.011 & -6.839 & -905.254 \\
\hline STORY4 & DEAD & 114.000 & -2637.480 & -6.839 & -125.647 \\
\hline STORY4 & DEAD & 228.000 & -2596.949 & -6.839 & 653.959 \\
\hline STORY4 & LIVE & 0.000 & -311.040 & -1.673 & -233.010 \\
\hline STORY4 & LIVE & 114.000 & -311.040 & -1.673 & -42.269 \\
\hline STORY4 & LIVE & 228.000 & -311.040 & -1.673 & 148.472 \\
\hline STORY4 & WIND & 0.000 & 7486.163 & 264.499 & 42595.510 \\
\hline STORY4 & WIND & 114.000 & 7486.163 & 264.499 & 12442.660 \\
\hline STORY4 & WIND & 228.000 & 7486.163 & 264.499 & -17710.200 \\
\hline STORY3 & DEAD & 0.000 & -2851.823 & -6.141 & -849.906 \\
\hline STORY3 & DEAD & 119.000 & -2804.061 & -6.141 & -119.121 \\
\hline STORY3 & DEAD & 238.000 & -2756.299 & -6.141 & 611.664 \\
\hline STORY3 & LIVE & 0.000 & -322.560 & -1.691 & -230.805 \\
\hline STORY3 & LIVE & 119.000 & -322.560 & -1.691 & -29.631 \\
\hline STORY3 & LIVE & 238.000 & -322.560 & -1.691 & 171.544 \\
\hline STORY3 & WIND & 0.000 & 7885.339 & 272.261 & 63358.860 \\
\hline STORY3 & WIND & 119.000 & 7885.339 & 272.261 & 30959.790 \\
\hline STORY3 & WIND & 238.000 & 7885.339 & 272.261 & -1439.280 \\
\hline STORY2 & DEAD & 0.000 & -3039.636 & -6.855 & -988.579 \\
\hline STORY2 & DEAD & 119.000 & -2986.089 & -6.855 & -172.879 \\
\hline STORY2 & DEAD & 238.000 & -2932.542 & -6.855 & 642.821 \\
\hline STORY2 & LIVE & 0.000 & -334.080 & -1.831 & -264.970 \\
\hline STORY2 & LIVE & 119.000 & -334.080 & -1.831 & -47.030 \\
\hline STORY2 & LIVE & 238.000 & -334.080 & -1.831 & 170.910 \\
\hline STORY2 & WIND & 0.000 & 8265.627 & 279.335 & 88946.340 \\
\hline STORY2 & WIND & 119.000 & 8265.627 & 279.335 & 55705.430 \\
\hline STORY2 & WIND & 238.000 & 8265.627 & 279.335 & 22464.530 \\
\hline STORY1 & DEAD & 0.000 & -3242.249 & -4.198 & -362.616 \\
\hline STORY1 & DEAD & 119.000 & -3182.587 & -4.198 & 136.975 \\
\hline STORY1 & DEAD & 238.000 & -3122.926 & -4.198 & 636.565 \\
\hline STORY1 & LIVE & 0.000 & -345.600 & -1.131 & -97.666 \\
\hline STORY1 & LIVE & 119.000 & -345.600 & -1.131 & 36.892 \\
\hline STORY1 & LIVE & 238.000 & -345.600 & -1.131 & 171.450 \\
\hline STORY1 & WIND & 0.000 & 8525.666 & 285.845 & 133777.300 \\
\hline STORY 1 & WIND & 119.000 & 8525.666 & 285.845 & 99761.670 \\
\hline STORY1 & WIND & 238.000 & 8525.666 & 285.845 & 65746.100 \\
\hline STORY30 & DEAD & 0.000 & -66.326 & -0.669 & -95.505 \\
\hline STORY30 & DEAD & 119.000 & -62.979 & -0.669 & -15.887 \\
\hline STORY30 & DEAD & 238.000 & -59.633 & -0.669 & 63.731 \\
\hline STORY30 & LIVE & 0.000 & -11.520 & -0.180 & -25.657 \\
\hline STORY30 & LIVE & 119.000 & -11.520 & -0.180 & -4.268 \\
\hline STORY30 & LIVE & 238.000 & -11.520 & -0.180 & 17.121 \\
\hline STORY30 & WIND & 0.000 & 5.895 & 5.823 & 831.138 \\
\hline STORY30 & WIND & 119.000 & 5.895 & 5.823 & 138.192 \\
\hline STORY30 & WIND & 238.000 & 5.895 & 5.823 & -554.754 \\
\hline STORY29 & DEAD & 0.000 & -132.652 & -0.631 & -89.811 \\
\hline STORY29 & DEAD & 119.000 & -129.305 & -0.631 & -14.750 \\
\hline
\end{tabular}




\begin{tabular}{|c|c|c|c|c|c|}
\hline STORY29 & DEAD & 238.000 & -125.959 & -0.631 & 60.311 \\
\hline STORY29 & LIVE & 0.000 & -23.040 & -0.169 & -24.128 \\
\hline STORY29 & LIVE & 119.000 & -23.040 & -0.169 & -3.963 \\
\hline STORY29 & LIVE & 238.000 & -23.040 & -0.169 & 16.203 \\
\hline STORY29 & WIND & 0.000 & 29.134 & 17.401 & 2499.954 \\
\hline STORY29 & WIND & 119.000 & 29.134 & 17.401 & 429.189 \\
\hline STORY29 & WIND & 238.000 & 29.134 & 17.401 & $-1641.5 \overline{77}$ \\
\hline STORY28 & DEAD & 0.000 & -200.878 & -1.423 & -202.660 \\
\hline STORY28 & DEAD & 119.000 & -196.746 & -1.423 & -33.371 \\
\hline STORY28 & DEAD & 238.000 & -192.614 & -1.423 & 135.919 \\
\hline STORY28 & LIVE & 0.000 & -34.560 & -0.382 & -54.445 \\
\hline STORY28 & LIVE & 119.000 & -34.560 & -0.382 & -8.965 \\
\hline STORY28 & LIVE & 238.000 & -34.560 & -0.382 & 36.515 \\
\hline STORY28 & WIND & 0.000 & 75.475 & 28.884 & 4149.901 \\
\hline STORY28 & WIND & 119.000 & 75.475 & 28.884 & 712.717 \\
\hline STORY28 & WIND & 238.000 & 75.475 & 28.884 & -2724.468 \\
\hline STORY27 & DEAD & 0.000 & -269.104 & -1.309 & -185.248 \\
\hline STORY27 & DEAD & 119.000 & -264.972 & -1.309 & -29.423 \\
\hline STORY27 & DEAD & 238.000 & -260.840 & -1.309 & 126.401 \\
\hline STORY27 & LIVE & 0.000 & -46.080 & -0.352 & -49.767 \\
\hline STORY27 & LIVE & 119.000 & -46.080 & -0.352 & -7.905 \\
\hline STORY27 & LIVE & 238.000 & -46.080 & -0.352 & 33.958 \\
\hline STORY27 & WIND & 0.000 & 144.549 & 40.263 & 5804.564 \\
\hline STORY27 & WIND & 119.000 & 144.549 & 40.263 & 1013.271 \\
\hline STORY27 & WIND & 238.000 & 144.549 & 40.263 & -3778.022 \\
\hline STORY26 & DEAD & 0.000 & -341.729 & -2.436 & -344.711 \\
\hline STORY26 & DEAD & 119.000 & -335.779 & -2.436 & -54.868 \\
\hline STORY26 & DEAD & 238.000 & -329.830 & -2.436 & 234.976 \\
\hline STORY26 & LIVE & 0.000 & -57.600 & -0.654 & -92.607 \\
\hline STORY26 & LIVE & 119.000 & -57.600 & -0.654 & -14.740 \\
\hline STORY26 & LIVE & 238.000 & -57.600 & -0.654 & 63.126 \\
\hline STORY26 & WIND & 0.000 & 236.289 & 51.546 & 7446.715 \\
\hline STORY26 & WIND & 119.000 & 236.289 & 51.546 & 1312.757 \\
\hline STORY26 & WIND & 238.000 & 236.289 & 51.546 & -4821.201 \\
\hline STORY25 & DEAD & 0.000 & -414.355 & -2.245 & -320.026 \\
\hline STORY25 & DEAD & 119.000 & -408.405 & -2.245 & -52.836 \\
\hline STORY25 & DEAD & 238.000 & -402.455 & -2.245 & 214.354 \\
\hline STORY25 & LIVE & 0.000 & -69.120 & -0.603 & -85.975 \\
\hline STORY25 & LIVE & 119.000 & -69.120 & -0.603 & -14.194 \\
\hline STORY25 & LIVE & 238.000 & -69.120 & -0.603 & 57.586 \\
\hline STORY25 & WIND & 0.000 & 350.351 & 62.718 & 9093.874 \\
\hline STORY25 & WIND & 119.000 & 350.351 & 62.718 & 1630.487 \\
\hline STORY25 & WIND & 238.000 & 350.351 & 62.718 & -5832.899 \\
\hline STORY24 & DEAD & 0.000 & -489.480 & -2.901 & -413.326 \\
\hline STORY24 & DEAD & 119.000 & -482.497 & -2.901 & -68.145 \\
\hline STORY24 & DEAD & 238.000 & -475.515 & -2.901 & 277.036 \\
\hline STORY24 & LIVE & 0.000 & -80.640 & -0.779 & -111.040 \\
\hline
\end{tabular}




\begin{tabular}{|c|c|c|c|c|c|}
\hline STORY24 & LIVE & 119.000 & -80.640 & -0.779 & -18.307 \\
\hline STORY24 & LIVE & 238.000 & -80.640 & -0.779 & 74.426 \\
\hline STORY24 & WIND & 0.000 & 486.569 & 73.789 & 10741.810 \\
\hline STORY24 & WIND & 119.000 & 486.569 & 73.789 & 1960.957 \\
\hline STORY24 & WIND & 238.000 & 486.569 & 73.789 & -6819.891 \\
\hline STORY23 & DEAD & 0.000 & -564.605 & -2.728 & -388.667 \\
\hline STORY23 & DEAD & 119.000 & -557.623 & -2.728 & -64.068 \\
\hline STORY23 & DEAD & 238.000 & -550.640 & -2.728 & 260.531 \\
\hline STORY23 & LIVE & 0.000 & -92.160 & -0.733 & -104.416 \\
\hline STORY23 & LIVE & 119.000 & -92.160 & -0.733 & -17.212 \\
\hline STORY23 & LIVE & 238.000 & -92.160 & -0.733 & 69.992 \\
\hline STORY23 & WIND & 0.000 & 644.771 & 84.744 & 12382.580 \\
\hline STORY23 & WIND & 119.000 & 644.771 & 84.744 & 2298.047 \\
\hline STORY23 & WIND & 238.000 & 644.771 & 84.744 & -7786.491 \\
\hline STORY22 & DEAD & 0.000 & -642.431 & -3.286 & -460.528 \\
\hline STORY22 & DEAD & 119.000 & -634.333 & -3.286 & -69.484 \\
\hline STORY22 & DEAD & 238.000 & -626.234 & -3.286 & 321.560 \\
\hline STORY22 & LIVE & 0.000 & -103.680 & -0.883 & -123.721 \\
\hline STORY22 & LIVE & 119.000 & -103.680 & -0.883 & -18.667 \\
\hline STORY22 & LIVE & 238.000 & -103.680 & -0.883 & 86.387 \\
\hline STORY22 & WIND & 0.000 & 824.576 & 95.598 & 14043.190 \\
\hline STORY22 & WIND & 119.000 & 824.576 & 95.598 & 2667.040 \\
\hline STORY22 & WIND & 238.000 & 824.576 & 95.598 & -8709.107 \\
\hline STORY21 & DEAD & 0.000 & -723.156 & -3.852 & -548.468 \\
\hline STORY21 & DEAD & 119.000 & -713.859 & -3.852 & -90.122 \\
\hline STORY21 & DEAD & 238.000 & -704.563 & -3.852 & 368.223 \\
\hline STORY21 & LIVE & 0.000 & -115.200 & -1.035 & -147.346 \\
\hline STORY21 & LIVE & 119.000 & -115.200 & -1.035 & -24.211 \\
\hline STORY21 & LIVE & 238.000 & -115.200 & -1.035 & 98.923 \\
\hline STORY21 & WIND & 0.000 & 1025.813 & 106.343 & 15719.540 \\
\hline STORY21 & WIND & 119.000 & 1025.813 & 106.343 & 3064.754 \\
\hline STORY21 & WIND & 238.000 & 1025.813 & 106.343 & -9590.030 \\
\hline STORY20 & DEAD & 0.000 & -803.881 & -3.626 & -517.070 \\
\hline STORY20 & DEAD & 119.000 & -794.584 & -3.626 & -85.621 \\
\hline STORY20 & DEAD & 238.000 & -785.288 & -3.626 & 345.828 \\
\hline STORY20 & LIVE & 0.000 & -126.720 & -0.974 & -138.911 \\
\hline STORY20 & LIVE & 119.000 & -126.720 & -0.974 & -23.002 \\
\hline STORY20 & LIVE & 238.000 & -126.720 & -0.974 & 92.907 \\
\hline STORY20 & WIND & 0.000 & 1248.519 & 116.956 & 17368.910 \\
\hline STORY20 & WIND & 119.000 & 1248.519 & 116.956 & 3451.119 \\
\hline STORY20 & WIND & 238.000 & 1248.519 & 116.956 & -10466.680 \\
\hline STORY19 & DEAD & 0.000 & -887.705 & -3.996 & -548.259 \\
\hline STORY19 & DEAD & 119.000 & -877.128 & -3.996 & -72.712 \\
\hline STORY19 & DEAD & 238.000 & -866.551 & -3.996 & 402.835 \\
\hline STORY19 & LIVE & 0.000 & -138.240 & -1.074 & -147.290 \\
\hline STORY19 & LIVE & 119.000 & -138.240 & -1.074 & -19.534 \\
\hline STORY 19 & LIVE & 238.000 & -138.240 & -1.074 & 108.222 \\
\hline
\end{tabular}




\begin{tabular}{|c|c|c|c|c|c|}
\hline STORY19 & WIND & 0.000 & 1491.870 & 127.471 & 19084.380 \\
\hline STORY19 & WIND & 119.000 & 1491.870 & 127.471 & 3915.320 \\
\hline STORY19 & WIND & 238.000 & 1491.870 & 127.471 & -11253.750 \\
\hline STORY18 & DEAD & 0.000 & -978.330 & -5.132 & -721.730 \\
\hline STORY18 & DEAD & 119.000 & -964.943 & -5.132 & -111.070 \\
\hline STORY18 & DEAD & 238.000 & -951.557 & -5.132 & 499.590 \\
\hline STORY18 & LIVE & 0.000 & -149.760 & -1.379 & -193.893 \\
\hline STORY18 & LIVE & 119.000 & -149.760 & -1.379 & -29.839 \\
\hline STORY18 & LIVE & 238.000 & -149.760 & -1.379 & 134.215 \\
\hline STORY18 & WIND & 0.000 & 1755.459 & 137.911 & 20912.310 \\
\hline STORY18 & WIND & 119.000 & 1755.459 & 137.911 & 4500.916 \\
\hline STORY18 & WIND & 238.000 & 1755.459 & 137.911 & -11910.480 \\
\hline STORY17 & DEAD & 0.000 & -1068.954 & -4.428 & -619.603 \\
\hline STORY17 & DEAD & 119.000 & -1055.568 & -4.428 & -92.645 \\
\hline STORY17 & DEAD & 238.000 & -1042.181 & -4.428 & 434.314 \\
\hline STORY17 & LIVE & 0.000 & -161.280 & -1.190 & -166.457 \\
\hline STORY17 & LIVE & 119.000 & -161.280 & -1.190 & -24.889 \\
\hline STORY17 & LIVE & 238.000 & -161.280 & -1.190 & 116.679 \\
\hline STORY17 & WIND & 0.000 & 2040.427 & 148.149 & 22629.760 \\
\hline STORY17 & WIND & 119.000 & 2040.427 & 148.149 & 4999.994 \\
\hline STORY 17 & WIND & 238.000 & 2040.427 & 148.149 & -12629.770 \\
\hline STORY16 & DEAD & 0.000 & -1167.178 & -5.608 & -780.697 \\
\hline STORY16 & DEAD & 119.000 & -1150.651 & -5.608 & -113.331 \\
\hline STORY16 & DEAD & 238.000 & -1134.125 & -5.608 & 554.036 \\
\hline STORY16 & LIVE & 0.000 & -172.800 & -1.507 & -209.735 \\
\hline STORY16 & LIVE & 119.000 & -172.800 & -1.507 & -30.446 \\
\hline STORY16 & LIVE & 238.000 & -172.800 & -1.507 & 148.842 \\
\hline STORY16 & WIND & 0.000 & 2344.466 & 158.384 & 24576.880 \\
\hline STORY16 & WIND & 119.000 & 2344.466 & 158.384 & 5729.174 \\
\hline STORY16 & WIND & 238.000 & 2344.466 & 158.384 & -13118.530 \\
\hline STORY15 & DEAD & 0.000 & -1265.402 & -5.259 & -766.485 \\
\hline STORY15 & DEAD & 119.000 & -1248.875 & -5.259 & -140.661 \\
\hline STORY15 & DEAD & 238.000 & -1232.349 & -5.259 & 485.164 \\
\hline STORY 15 & LIVE & 0.000 & -184.320 & -1.413 & -205.917 \\
\hline STORY 15 & LIVE & 119.000 & -184.320 & -1.413 & -37.789 \\
\hline STORY 15 & LIVE & 238.000 & -184.320 & -1.413 & 130.340 \\
\hline STORY 15 & WIND & 0.000 & 2669.967 & 168.391 & 26349.060 \\
\hline STORY15 & WIND & 119.000 & 2669.967 & 168.391 & 6310.500 \\
\hline STORY15 & WIND & 238.000 & 2669.967 & 168.391 & -13728.060 \\
\hline STORY 14 & DEAD & 0.000 & -1363.626 & -4.941 & -686.142 \\
\hline STORY14 & DEAD & 119.000 & -1347.099 & -4.941 & -98.202 \\
\hline STORY14 & DEAD & 238.000 & -1330.572 & -4.941 & 489.738 \\
\hline STORY14 & LIVE & 0.000 & -195.840 & -1.327 & -184.333 \\
\hline STORY14 & LIVE & 119.000 & -195.840 & -1.327 & -26.382 \\
\hline STORY14 & LIVE & 238.000 & -195.840 & -1.327 & 131.569 \\
\hline STORY14 & WIND & 0.000 & 3015.326 & 178.260 & 28136.390 \\
\hline STORY 14 & WIND & 119.000 & 3015.326 & 178.260 & 6923.416 \\
\hline
\end{tabular}




\begin{tabular}{|c|c|c|c|c|c|}
\hline STORY14 & WIND & 238.000 & 3015.326 & 178.260 & -14289.560 \\
\hline STORY13 & DEAD & 0.000 & -1470.249 & -6.016 & -845.007 \\
\hline STORY13 & DEAD & 119.000 & -1450.252 & -6.016 & -129.125 \\
\hline STORY13 & DEAD & 238.000 & -1430.255 & -6.016 & 586.757 \\
\hline STORY13 & LIVE & 0.000 & -207.360 & -1.616 & -227.014 \\
\hline STORY13 & LIVE & 119.000 & -207.360 & -1.616 & -34.690 \\
\hline STORY13 & LIVE & 238.000 & -207.360 & -1.616 & 157.633 \\
\hline STORY13 & WIND & 0.000 & 3378.294 & 188.212 & 30313.870 \\
\hline STORY13 & WIND & 119.000 & 3378.294 & 188.212 & 7916.615 \\
\hline STORY13 & WIND & 238.000 & 3378.294 & 188.212 & -14480.640 \\
\hline STORY12 & DEAD & 0.000 & -1576.873 & -5.198 & -727.843 \\
\hline STORY12 & DEAD & 119.000 & -1556.875 & -5.198 & -109.309 \\
\hline STORY12 & DEAD & 238.000 & -1536.878 & -5.198 & 509.225 \\
\hline STORY12 & LIVE & 0.000 & -218.880 & -1.396 & -195.520 \\
\hline STORY 12 & LIVE & 119.000 & -218.880 & -1.396 & -29.359 \\
\hline STORY12 & LIVE & 238.000 & -218.880 & -1.396 & 136.802 \\
\hline STORY12 & WIND & 0.000 & 3762.442 & 197.810 & 32271.510 \\
\hline STORY12 & WIND & 119.000 & 3762.442 & 197.810 & 8732.102 \\
\hline STORY12 & WIND & 238.000 & 3762.442 & 197.810 & -14807.300 \\
\hline STORY11 & DEAD & 0.000 & -1692.696 & -6.267 & -879.442 \\
\hline STORY11 & DEAD & 119.000 & -1668.898 & -6.267 & -133.672 \\
\hline STORY11 & DEAD & 238.000 & -1645.099 & -6.267 & 612.099 \\
\hline STORY11 & LIVE & 0.000 & -230.400 & -1.684 & -236.385 \\
\hline STORY11 & LIVE & 119.000 & -230.400 & -1.684 & -35.966 \\
\hline STORY11 & LIVE & 238.000 & -230.400 & -1.684 & 164.453 \\
\hline STORY11 & WIND & 0.000 & 4162.534 & 207.207 & 34708.120 \\
\hline STORY11 & WIND & 119.000 & 4162.534 & 207.207 & 10050.480 \\
\hline STORY11 & WIND & 238.000 & 4162.534 & 207.207 & -14607.170 \\
\hline STORY10 & DEAD & 0.000 & -1808.523 & -5.459 & -767.122 \\
\hline STORY10 & DEAD & 119.000 & -1784.725 & -5.459 & -117.556 \\
\hline STORY10 & DEAD & 238.000 & -1760.926 & -5.459 & 532.009 \\
\hline STORY10 & LIVE & 0.000 & -241.922 & -1.463 & -205.358 \\
\hline STORY10 & LIVE & 119.000 & -241.922 & -1.463 & -31.253 \\
\hline STORY10 & LIVE & 238.000 & -241.922 & -1.463 & 142.853 \\
\hline STORY10 & WIND & 0.000 & 4580.825 & 218.525 & 37895.390 \\
\hline STORY 10 & WIND & 119.000 & 4580.825 & 218.525 & 11890.970 \\
\hline STORY10 & WIND & 238.000 & 4580.825 & 218.525 & -14113.440 \\
\hline STORY9 & DEAD & 0.000 & -1934.368 & -6.316 & -877.484 \\
\hline STORY9 & DEAD & 119.000 & -1906.438 & -6.316 & -125.844 \\
\hline STORY9 & DEAD & 238.000 & -1878.508 & -6.316 & 625.796 \\
\hline STORY9 & LIVE & 0.000 & -253.450 & -1.718 & -240.505 \\
\hline STORY9 & LIVE & 119.000 & -253.450 & -1.718 & -36.039 \\
\hline STORY9 & LIVE & 238.000 & -253.450 & -1.718 & 168.428 \\
\hline STORY9 & WIND & 0.000 & 4984.345 & 207.791 & 39178.700 \\
\hline STORY9 & WIND & 119.000 & 4984.345 & 207.791 & 14451.610 \\
\hline STORY9 & WIND & 238.000 & 4984.345 & 207.791 & -10275.480 \\
\hline STORY 8 & DEAD & 0.000 & -2060.174 & -6.091 & -897.416 \\
\hline
\end{tabular}




\begin{tabular}{|c|c|c|c|c|c|}
\hline STORY8 & DEAD & 119.000 & -2032.243 & -6.091 & -172.540 \\
\hline STORY8 & DEAD & 238.000 & -2004.313 & -6.091 & 552.338 \\
\hline STORY8 & LIVE & 0.000 & -264.950 & -1.505 & -212.536 \\
\hline STORY8 & LIVE & 119.000 & -264.950 & -1.505 & -33.384 \\
\hline STORY8 & LIVE & 238.000 & -264.950 & -1.505 & 145.768 \\
\hline STORY8 & WIND & 0.000 & 5354.556 & 216.203 & 46166.760 \\
\hline STORY8 & WIND & 119.000 & 5354.556 & 216.203 & 20438.560 \\
\hline STORY8 & WIND & 238.000 & 5354.556 & 216.203 & -5289.639 \\
\hline STORY7 & DEAD & 0.000 & -2198.444 & -6.676 & -918.653 \\
\hline STORY7 & DEAD & 114.000 & -2169.589 & -6.676 & -157.631 \\
\hline STORY7 & DEAD & 228.000 & -2140.735 & -6.676 & 603.392 \\
\hline STORY7 & LIVE & 0.000 & -276.478 & -1.495 & -207.356 \\
\hline STORY7 & LIVE & 114.000 & -276.478 & -1.495 & -36.907 \\
\hline STORY7 & LIVE & 228.000 & -276.478 & -1.495 & 133.542 \\
\hline STORY7 & WIND & 0.000 & 5848.986 & 246.001 & 45556.540 \\
\hline STORY7 & WIND & 114.000 & 5848.986 & 246.001 & 17512.460 \\
\hline STORY7 & WIND & 228.000 & 5848.986 & 246.001 & -10531.610 \\
\hline STORY6 & DEAD & 0.000 & -2347.899 & -7.099 & -989.984 \\
\hline STORY6 & DEAD & 114.000 & -2314.612 & -7.099 & -180.664 \\
\hline STORY6 & DEAD & 228.000 & -2281.325 & -7.099 & 628.656 \\
\hline STORY6 & LIVE & 0.000 & -287.999 & -1.626 & -226.728 \\
\hline STORY6 & LIVE & 114.000 & -287.999 & -1.626 & -41.348 \\
\hline STORY6 & LIVE & 228.000 & -287.999 & -1.626 & 144.031 \\
\hline STORY6 & WIND & 0.000 & 6340.711 & 250.866 & 47494.000 \\
\hline STORY6 & WIND & 114.000 & 6340.711 & 250.866 & 18895.230 \\
\hline STORY6 & WIND & 228.000 & 6340.711 & 250.866 & -9703.536 \\
\hline STORY5 & DEAD & 0.000 & -2503.251 & -6.943 & -975.809 \\
\hline STORY5 & DEAD & 114.000 & -2467.628 & -6.943 & -184.349 \\
\hline STORY5 & DEAD & 228.000 & -2432.005 & -6.943 & 607.112 \\
\hline STORY5 & LIVE & 0.000 & -299.520 & -1.558 & -217.258 \\
\hline STORY5 & LIVE & 114.000 & -299.520 & -1.558 & -39.594 \\
\hline STORY5 & LIVE & 228.000 & -299.520 & -1.558 & 138.070 \\
\hline STORY5 & WIND & 0.000 & 6854.013 & 259.026 & 48532.080 \\
\hline STORY5 & WIND & 114.000 & 6854.013 & 259.026 & 19003.160 \\
\hline STORY5 & WIND & 228.000 & 6854.013 & 259.026 & -10525.760 \\
\hline STORY4 & DEAD & 0.000 & -2671.002 & -6.838 & -905.216 \\
\hline STORY4 & DEAD & 114.000 & -2630.471 & -6.838 & -125.692 \\
\hline STORY4 & DEAD & 228.000 & -2589.940 & -6.838 & 653.832 \\
\hline STORY4 & LIVE & 0.000 & -311.040 & -1.673 & -233.017 \\
\hline STORY4 & LIVE & 114.000 & -311.040 & -1.673 & -42.263 \\
\hline STORY4 & LIVE & 228.000 & -311.040 & -1.673 & 148.491 \\
\hline STORY4 & WIND & 0.000 & 7428.333 & 266.166 & 42745.370 \\
\hline STORY4 & WIND & 114.000 & 7428.333 & 266.166 & 12402.430 \\
\hline STORY4 & WIND & 228.000 & 7428.333 & 266.166 & -17940.500 \\
\hline STORY3 & DEAD & 0.000 & -2844.827 & -6.141 & -849.931 \\
\hline STORY3 & DEAD & 119.000 & -2797.065 & -6.141 & -119.111 \\
\hline STORY3 & DEAD & 238.000 & -2749.303 & -6.141 & 611.709 \\
\hline
\end{tabular}




\begin{tabular}{|c|c|c|c|c|c|}
\hline STORY3 & LIVE & 0.000 & -322.560 & -1.691 & -230.803 \\
\hline STORY3 & LIVE & 119.000 & -322.560 & -1.691 & -29.632 \\
\hline STORY3 & LIVE & 238.000 & -322.560 & -1.691 & 171.539 \\
\hline STORY3 & WIND & 0.000 & 7831.847 & 273.092 & 63456.180 \\
\hline STORY3 & WIND & 119.000 & 7831.847 & 273.092 & 30958.220 \\
\hline STORY3 & WIND & 238.000 & 7831.847 & 273.092 & -1539.748 \\
\hline STORY2 & DEAD & 0.000 & -3032.651 & -6.855 & -988.587 \\
\hline STORY2 & DEAD & 119.000 & -2979.105 & -6.855 & -172.883 \\
\hline STORY2 & DEAD & 238.000 & -2925.558 & -6.855 & 642.821 \\
\hline STORY2 & LIVE & 0.000 & -334.080 & -1.831 & -264.970 \\
\hline STORY2 & LIVE & 119.000 & -334.080 & -1.831 & -47.030 \\
\hline STORY2 & LIVE & 238.000 & -334.080 & -1.831 & 170.911 \\
\hline STORY2 & WIND & 0.000 & 8214.807 & 279.838 & 89002.780 \\
\hline STORY2 & WIND & 119.000 & 8214.807 & 279.838 & 55702.120 \\
\hline STORY2 & WIND & 238.000 & 8214.807 & 279.838 & 22401.460 \\
\hline STORY1 & DEAD & 0.000 & -3235.273 & -4.198 & -362.620 \\
\hline STORY1 & DEAD & 119.000 & -3175.611 & -4.198 & 136.976 \\
\hline STORY1 & DEAD & 238.000 & -3115.950 & -4.198 & 636.573 \\
\hline STORY1 & LIVE & 0.000 & -345.600 & -1.131 & -97.666 \\
\hline STORY1 & LIVE & 119.000 & -345.600 & -1.131 & 36.892 \\
\hline STORY1 & LIVE & 238.000 & -345.600 & -1.131 & 171.450 \\
\hline STORY1 & WIND & 0.000 & 8476.116 & 286.004 & 133790.400 \\
\hline STORY1 & WIND & 119.000 & 8476.116 & 286.004 & 99755.880 \\
\hline STORY1 & WIND & 238.000 & 8476.116 & 286.004 & 65721.380 \\
\hline STORY30 & DEAD & 0.000 & -66.326 & 0.669 & 95.505 \\
\hline STORY30 & DEAD & 119.000 & -62.979 & 0.669 & 15.887 \\
\hline STORY30 & DEAD & 238.000 & -59.633 & 0.669 & -63.731 \\
\hline STORY30 & LIVE & 0.000 & -11.520 & 0.180 & 25.657 \\
\hline STORY30 & LIVE & 119.000 & -11.520 & 0.180 & 4.268 \\
\hline STORY30 & LIVE & 238.000 & -11.520 & 0.180 & -17.121 \\
\hline STORY30 & WIND & 0.000 & -5.850 & 5.820 & 830.666 \\
\hline STORY30 & WIND & 119.000 & -5.850 & 5.820 & 138.105 \\
\hline STORY30 & WIND & 238.000 & -5.850 & 5.820 & -554.456 \\
\hline STORY29 & DEAD & 0.000 & -132.652 & 0.631 & 89.811 \\
\hline STORY29 & DEAD & 119.000 & -129.305 & 0.631 & 14.750 \\
\hline STORY29 & DEAD & 238.000 & -125.959 & 0.631 & -60.311 \\
\hline STORY29 & LIVE & 0.000 & -23.040 & 0.169 & 24.128 \\
\hline STORY29 & LIVE & 119.000 & -23.040 & 0.169 & 3.963 \\
\hline STORY 29 & LIVE & 238.000 & -23.040 & 0.169 & -16.203 \\
\hline STORY29 & WIND & 0.000 & -29.032 & 17.398 & 2499.415 \\
\hline STORY29 & WIND & 119.000 & -29.032 & 17.398 & 429.083 \\
\hline STORY29 & WIND & 238.000 & -29.032 & 17.398 & -1641.248 \\
\hline STORY28 & DEAD & 0.000 & -200.878 & 1.423 & 202.660 \\
\hline STORY28 & DEAD & 119.000 & -196.746 & 1.423 & 33.371 \\
\hline STORY 28 & DEAD & 238.000 & -192.614 & 1.423 & -135.919 \\
\hline STORY28 & LIVE & 0.000 & -34.560 & 0.382 & 54.445 \\
\hline STORY28 & LIVE & 119.000 & -34.560 & 0.382 & 8.965 \\
\hline
\end{tabular}




\begin{tabular}{|c|c|c|c|c|c|}
\hline STORY28 & LIVE & 238.000 & -34.560 & 0.382 & -36.515 \\
\hline STORY28 & WIND & 0.000 & -75.297 & 28.874 & 4148.424 \\
\hline STORY28 & WIND & 119.000 & -75.297 & 28.874 & 712.437 \\
\hline STORY28 & WIND & 238.000 & -75.297 & 28.874 & -2723.551 \\
\hline STORY27 & DEAD & 0.000 & -269.104 & 1.309 & 185.248 \\
\hline STORY27 & DEAD & 119.000 & -264.972 & 1.309 & 29.423 \\
\hline STORY27 & DEAD & 238.000 & -260.840 & 1.309 & -126.401 \\
\hline STORY27 & LIVE & 0.000 & -46.080 & 0.352 & 49.767 \\
\hline STORY27 & LIVE & 119.000 & -46.080 & 0.352 & 7.905 \\
\hline STORY27 & LIVE & 238.000 & -46.080 & 0.352 & -33.958 \\
\hline STORY27 & WIND & 0.000 & -144.272 & 40.252 & 5802.874 \\
\hline STORY27 & WIND & 119.000 & -144.272 & 40.252 & 1012.945 \\
\hline STORY27 & WIND & 238.000 & -144.272 & 40.252 & -3776.984 \\
\hline STORY26 & DEAD & 0.000 & -341.729 & 2.436 & 344.711 \\
\hline STORY26 & DEAD & 119.000 & -335.779 & 2.436 & 54.868 \\
\hline STORY26 & DEAD & 238.000 & -329.830 & 2.436 & -234.976 \\
\hline STORY26 & LIVE & 0.000 & -57.600 & 0.654 & 92.607 \\
\hline STORY26 & LIVE & 119.000 & -57.600 & 0.654 & 14.740 \\
\hline STORY26 & LIVE & 238.000 & -57.600 & 0.654 & -63.126 \\
\hline STORY26 & WIND & 0.000 & -235.870 & 51.519 & 7442.750 \\
\hline STORY26 & WIND & 119.000 & -235.870 & 51.519 & 1312.001 \\
\hline STORY26 & WIND & 238.000 & -235.870 & 51.519 & -4818.747 \\
\hline STORY25 & DEAD & 0.000 & -414.355 & 2.245 & 320.026 \\
\hline STORY25 & DEAD & 119.000 & -408.405 & 2.245 & 52.836 \\
\hline STORY25 & DEAD & 238.000 & -402.455 & 2.245 & -214.354 \\
\hline STORY25 & LIVE & 0.000 & -69.120 & 0.603 & 85.975 \\
\hline STORY25 & LIVE & 119.000 & -69.120 & 0.603 & 14.194 \\
\hline STORY25 & LIVE & 238.000 & -69.120 & 0.603 & -57.586 \\
\hline STORY25 & WIND & 0.000 & -349.743 & 62.686 & 9089.242 \\
\hline STORY25 & WIND & 119.000 & -349.743 & 62.686 & 1629.576 \\
\hline STORY25 & WIND & 238.000 & -349.743 & 62.686 & -5830.089 \\
\hline STORY24 & DEAD & 0.000 & -489.480 & 2.901 & 413.326 \\
\hline STORY24 & DEAD & 119.000 & -482.497 & 2.901 & 68.145 \\
\hline STORY24 & DEAD & 238.000 & -475.515 & 2.901 & -277.036 \\
\hline STORY24 & LIVE & 0.000 & -80.640 & 0.779 & 111.040 \\
\hline STORY24 & LIVE & 119.000 & -80.640 & 0.779 & 18.307 \\
\hline STORY24 & LIVE & 238.000 & -80.640 & 0.779 & -74.426 \\
\hline STORY24 & WIND & 0.000 & -485.710 & 73.738 & 10734.270 \\
\hline STORY24 & WIND & 119.000 & -485.710 & 73.738 & 1959.476 \\
\hline STORY24 & WIND & 238.000 & -485.710 & 73.738 & -6815.323 \\
\hline STORY23 & DEAD & 0.000 & -564.605 & 2.728 & 388.667 \\
\hline STORY23 & DEAD & 119.000 & -557.623 & 2.728 & 64.068 \\
\hline STORY23 & DEAD & 238.000 & -550.640 & 2.728 & -260.531 \\
\hline STORY23 & LIVE & 0.000 & -92.160 & 0.733 & 104.416 \\
\hline STORY23 & LIVE & 119.000 & -92.160 & 0.733 & 17.212 \\
\hline STORY23 & LIVE & 238.000 & -92.160 & 0.733 & -69.992 \\
\hline STORY23 & WIND & 0.000 & -643.585 & 84.683 & 12373.550 \\
\hline
\end{tabular}




\begin{tabular}{|c|c|c|c|c|c|}
\hline STORY23 & WIND & 119.000 & -643.585 & 84.683 & 2296.248 \\
\hline STORY23 & WIND & 238.000 & -643.585 & 84.683 & -7781.054 \\
\hline STORY22 & DEAD & 0.000 & -642.431 & 3.286 & 460.528 \\
\hline STORY22 & DEAD & 119.000 & -634.333 & 3.286 & 69.484 \\
\hline STORY22 & DEAD & 238.000 & -626.234 & 3.286 & -321.560 \\
\hline STORY22 & LIVE & 0.000 & -103.680 & 0.883 & 123.721 \\
\hline STORY22 & LIVE & 119.000 & -103.680 & 0.883 & 18.667 \\
\hline STORY22 & LIVE & 238.000 & -103.680 & 0.883 & -86.387 \\
\hline STORY22 & WIND & 0.000 & -822.951 & 95.502 & 14029.120 \\
\hline STORY22 & WIND & 119.000 & -822.951 & 95.502 & 2664.336 \\
\hline STORY22 & WIND & 238.000 & -822.951 & 95.502 & -8700.447 \\
\hline STORY21 & DEAD & 0.000 & -723.156 & 3.852 & 548.468 \\
\hline STORY21 & DEAD & 119.000 & -713.859 & 3.852 & 90.122 \\
\hline STORY21 & DEAD & 238.000 & -704.563 & 3.852 & -368.223 \\
\hline STORY21 & LIVE & 0.000 & -115.200 & 1.035 & 147.346 \\
\hline STORY21 & LIVE & 119.000 & -115.200 & 1.035 & 24.211 \\
\hline STORY21 & LIVE & 238.000 & -115.200 & 1.035 & -98.923 \\
\hline STORY21 & WIND & 0.000 & -1023.563 & 106.197 & 15697.870 \\
\hline STORY21 & WIND & 119.000 & -1023.563 & 106.197 & 3060.485 \\
\hline STORY21 & WIND & 238.000 & -1023.563 & 106.197 & -9576.903 \\
\hline STORY20 & DEAD & 0.000 & -803.881 & 3.626 & 517.070 \\
\hline STORY20 & DEAD & 119.000 & -794.584 & 3.626 & 85.621 \\
\hline STORY20 & DEAD & 238.000 & -785.288 & 3.626 & -345.828 \\
\hline STORY20 & LIVE & 0.000 & -126.720 & 0.974 & 138.911 \\
\hline STORY20 & LIVE & 119.000 & -126.720 & 0.974 & 23.002 \\
\hline STORY20 & LIVE & 238.000 & -126.720 & 0.974 & -92.907 \\
\hline STORY20 & WIND & 0.000 & -1245.454 & 116.781 & 17342.880 \\
\hline STORY20 & WIND & 119.000 & -1245.454 & 116.781 & 3445.924 \\
\hline STORY20 & WIND & 238.000 & -1245.454 & 116.781 & -10451.030 \\
\hline STORY19 & DEAD & 0.000 & -887.705 & 3.996 & 548.259 \\
\hline STORY19 & DEAD & 119.000 & -877.128 & 3.996 & 72.712 \\
\hline STORY19 & DEAD & 238.000 & -866.551 & 3.996 & -402.835 \\
\hline STORY19 & LIVE & 0.000 & -138.240 & 1.074 & 147.290 \\
\hline STORY19 & LIVE & 119.000 & -138.240 & 1.074 & 19.534 \\
\hline STORY19 & LIVE & 238.000 & -138.240 & 1.074 & -108.222 \\
\hline STORY19 & WIND & 0.000 & -1487.728 & 127.216 & 19047.290 \\
\hline STORY19 & WIND & 119.000 & -1487.728 & 127.216 & 3908.557 \\
\hline STORY19 & WIND & 238.000 & -1487.728 & 127.216 & -11230.180 \\
\hline STORY18 & DEAD & 0.000 & -978.330 & 5.132 & 721.730 \\
\hline STORY 18 & DEAD & 119.000 & -964.943 & 5.132 & 111.070 \\
\hline STORY 18 & DEAD & 238.000 & -951.557 & 5.132 & -499.590 \\
\hline STORY18 & LIVE & 0.000 & -149.760 & 1.379 & 193.893 \\
\hline STORY 18 & LIVE & 119.000 & -149.760 & 1.379 & 29.839 \\
\hline STORY 18 & LIVE & 238.000 & -149.760 & 1.379 & -134.215 \\
\hline STORY18 & WIND & 0.000 & -1749.705 & 137.470 & 20847.700 \\
\hline STORY18 & WIND & 119.000 & -1749.705 & 137.470 & 4488.725 \\
\hline STORY 18 & WIND & 238.000 & -1749.705 & 137.470 & -11870.250 \\
\hline
\end{tabular}




\begin{tabular}{|c|c|c|c|c|c|}
\hline STORY17 & DEAD & 0.000 & -1068.954 & 4.428 & 619.603 \\
\hline STORY17 & DEAD & 119.000 & -1055.568 & 4.428 & 92.645 \\
\hline STORY17 & DEAD & 238.000 & -1042.181 & 4.428 & -434.314 \\
\hline STORY17 & LIVE & 0.000 & -161.280 & 1.190 & 166.457 \\
\hline STORY17 & LIVE & 119.000 & -161.280 & 1.190 & 24.889 \\
\hline STORY17 & LIVE & 238.000 & -161.280 & 1.190 & -116.679 \\
\hline STORY17 & WIND & 0.000 & -2032.553 & 147.661 & 22558.210 \\
\hline STORY17 & WIND & 119.000 & -2032.553 & 147.661 & 4986.564 \\
\hline STORY17 & WIND & 238.000 & -2032.553 & 147.661 & -12585.090 \\
\hline STORY16 & DEAD & 0.000 & -1167.178 & 5.608 & 780.697 \\
\hline STORY16 & DEAD & 119.000 & -1150.651 & 5.608 & 113.331 \\
\hline STORY16 & DEAD & 238.000 & -1134.125 & 5.608 & -554.036 \\
\hline STORY16 & LIVE & 0.000 & -172.800 & 1.507 & 209.735 \\
\hline STORY16 & LIVE & 119.000 & -172.800 & 1.507 & 30.446 \\
\hline STORY16 & LIVE & 238.000 & -172.800 & 1.507 & -148.842 \\
\hline STORY16 & WIND & 0.000 & -2333.716 & 157.581 & 24459.700 \\
\hline STORY16 & WIND & 119.000 & -2333.716 & 157.581 & 5707.589 \\
\hline STORY16 & WIND & 238.000 & -2333.716 & 157.581 & -13044.520 \\
\hline STORY15 & DEAD & 0.000 & -1265.402 & 5.259 & 766.487 \\
\hline STORY15 & DEAD & 119.000 & -1248.875 & 5.259 & 140.662 \\
\hline STORY15 & DEAD & 238.000 & -1232.349 & 5.259 & -485.164 \\
\hline STORY15 & LIVE & 0.000 & -184.320 & 1.413 & 205.917 \\
\hline STORY15 & LIVE & 119.000 & -184.320 & 1.413 & 37.789 \\
\hline STORY15 & LIVE & 238.000 & -184.320 & 1.413 & -130.340 \\
\hline STORY15 & WIND & 0.000 & -2655.446 & 167.443 & 26206.720 \\
\hline STORY15 & WIND & 119.000 & -2655.446 & 167.443 & 6280.972 \\
\hline STORY15 & WIND & 238.000 & -2655.446 & 167.443 & -13644.780 \\
\hline STORY14 & DEAD & 0.000 & -1363.626 & 4.941 & 686.130 \\
\hline STORY14 & DEAD & 119.000 & -1347.099 & 4.941 & 98.196 \\
\hline STORY14 & DEAD & 238.000 & -1330.572 & 4.941 & -489.737 \\
\hline STORY14 & LIVE & 0.000 & -195.840 & 1.327 & 184.334 \\
\hline STORY14 & LIVE & 119.000 & -195.840 & 1.327 & 26.383 \\
\hline STORY14 & LIVE & 238.000 & -195.840 & 1.327 & -131.569 \\
\hline STORY14 & WIND & 0.000 & -2996.220 & 177.148 & 27973.630 \\
\hline STORY14 & WIND & 119.000 & -2996.220 & 177.148 & 6893.083 \\
\hline STORY14 & WIND & 238.000 & -2996.220 & 177.148 & -14187.460 \\
\hline STORY13 & DEAD & 0.000 & -1470.249 & 6.016 & 845.089 \\
\hline STORY13 & DEAD & 119.000 & -1450.252 & 6.016 & 129.163 \\
\hline STORY13 & DEAD & 238.000 & -1430.255 & 6.016 & -586.763 \\
\hline STORY13 & LIVE & 0.000 & -207.360 & 1.616 & 227.003 \\
\hline STORY13 & LIVE & 119.000 & -207.360 & 1.616 & 34.685 \\
\hline STORY13 & LIVE & 238.000 & -207.360 & 1.616 & -157.632 \\
\hline STORY13 & WIND & 0.000 & -3352.998 & 186.459 & 30058.060 \\
\hline STORY13 & WIND & 119.000 & -3352.998 & 186.459 & 7869.419 \\
\hline STORY13 & WIND & 238.000 & -3352.998 & 186.459 & -14319.220 \\
\hline STORY12 & DEAD & 0.000 & -1576.872 & 5.196 & 727.393 \\
\hline STORY12 & DEAD & 119.000 & -1556.875 & 5.196 & 109.104 \\
\hline
\end{tabular}




\begin{tabular}{|c|c|c|c|c|c|}
\hline STORY12 & DEAD & 238.000 & -1536.878 & 5.196 & -509.185 \\
\hline STORY12 & LIVE & 0.000 & -218.880 & 1.397 & 195.582 \\
\hline STORY12 & LIVE & 119.000 & -218.880 & 1.397 & 29.387 \\
\hline STORY12 & LIVE & 238.000 & -218.880 & 1.397 & -136.807 \\
\hline STORY12 & WIND & 0.000 & -3729.166 & 195.801 & 31974.820 \\
\hline STORY12 & WIND & 119.000 & -3729.166 & 195.801 & 8674.538 \\
\hline STORY12 & WIND & 238.000 & -3729.166 & 195.801 & -14625.740 \\
\hline STORY11 & DEAD & 0.000 & -1692.694 & 6.279 & 882.220 \\
\hline STORY11 & DEAD & 119.000 & -1668.896 & 6.279 & 134.984 \\
\hline STORY11 & DEAD & 238.000 & -1645.098 & 6.279 & -612.252 \\
\hline STORY11 & LIVE & 0.000 & -230.400 & 1.682 & 236.002 \\
\hline STORY11 & LIVE & 119.000 & -230.400 & 1.682 & 35.786 \\
\hline STORY11 & LIVE & 238.000 & -230.400 & 1.682 & -164.431 \\
\hline STORY11 & WIND & 0.000 & -4119.290 & 205.002 & 34463.370 \\
\hline STORY11 & WIND & 119.000 & -4119.290 & 205.002 & 10068.100 \\
\hline STORY11 & WIND & 238.000 & -4119.290 & 205.002 & -14327.170 \\
\hline STORY10 & DEAD & 0.000 & -1808.513 & 5.395 & 752.966 \\
\hline STORY10 & DEAD & 119.000 & -1784.714 & 5.395 & 111.004 \\
\hline STORY10 & DEAD & 238.000 & -1760.916 & 5.395 & -530.959 \\
\hline STORY 10 & LIVE & 0.000 & -241.918 & 1.472 & 207.288 \\
\hline STORY10 & LIVE & 119.000 & -241.918 & 1.472 & 32.147 \\
\hline STORY10 & LIVE & 238.000 & -241.918 & 1.472 & -142.994 \\
\hline STORY10 & WIND & 0.000 & -4522.587 & 211.923 & 36936.360 \\
\hline STORY10 & WIND & 119.000 & -4522.587 & 211.923 & 11717.490 \\
\hline STORY10 & WIND & 238.000 & -4522.587 & 211.923 & -13501.370 \\
\hline STORY9 & DEAD & 0.000 & -1934.313 & 6.674 & 959.145 \\
\hline STORY9 & DEAD & 119.000 & -1906.382 & 6.674 & 164.949 \\
\hline STORY9 & DEAD & 238.000 & -1878.452 & 6.674 & -629.247 \\
\hline STORY9 & LIVE & 0.000 & -253.431 & 1.669 & 229.258 \\
\hline STORY9 & LIVE & 119.000 & -253.431 & 1.669 & 30.662 \\
\hline STORY9 & LIVE & 238.000 & -253.431 & 1.669 & -167.935 \\
\hline STORY9 & WIND & 0.000 & -4922.562 & 240.513 & 49061.390 \\
\hline STORY9 & WIND & 119.000 & -4922.562 & 240.513 & 20440.340 \\
\hline STORY9 & WIND & 238.000 & -4922.562 & 240.513 & -8180.706 \\
\hline STORY8 & DEAD & 0.000 & -2067.276 & 6.476 & 924.026 \\
\hline STORY8 & DEAD & 114.000 & -2040.519 & 6.476 & 185.776 \\
\hline STORY8 & DEAD & 228.000 & -2013.763 & 6.476 & -552.473 \\
\hline STORY8 & LIVE & 0.000 & -264.970 & 1.452 & 208.856 \\
\hline STORY8 & LIVE & 114.000 & -264.970 & 1.452 & 43.273 \\
\hline STORY8 & LIVE & 228.000 & -264.970 & 1.452 & -122.310 \\
\hline STORY8 & WIND & 0.000 & -5458.718 & 249.547 & 45692.610 \\
\hline STORY 8 & WIND & 114.000 & -5458.718 & 249.547 & 17244.210 \\
\hline STORY8 & WIND & 228.000 & -5458.718 & 249.547 & -11204.180 \\
\hline STORY7 & DEAD & 0.000 & -2205.498 & 6.597 & 913.200 \\
\hline STORY7 & DEAD & 114.000 & -2176.644 & 6.597 & 161.105 \\
\hline STORY7 & DEAD & 228.000 & -2147.790 & 6.597 & -590.990 \\
\hline STORY7 & LIVE & 0.000 & -276.482 & 1.506 & 208.095 \\
\hline
\end{tabular}




\begin{tabular}{|c|c|c|c|c|c|}
\hline STORY7 & LIVE & 114.000 & -276.482 & 1.506 & 36.431 \\
\hline STORY7 & LIVE & 228.000 & -276.482 & 1.506 & -135.233 \\
\hline STORY7 & WIND & 0.000 & -5943.690 & 236.756 & 44302.780 \\
\hline STORY7 & WIND & 114.000 & -5943.690 & 236.756 & 17312.610 \\
\hline STORY7 & WIND & 228.000 & -5943.690 & 236.756 & -9677.565 \\
\hline STORY6 & DEAD & 0.000 & -2354.934 & 7.116 & 991.090 \\
\hline STORY6 & DEAD & 114.000 & -2321.646 & 7.116 & 179.839 \\
\hline STORY6 & DEAD & 228.000 & -2288.359 & 7.116 & -631.412 \\
\hline STORY6 & LIVE & 0.000 & -288.001 & 1.624 & 226.573 \\
\hline STORY6 & LIVE & 114.000 & -288.001 & 1.624 & 41.461 \\
\hline STORY6 & LIVE & 228.000 & -288.001 & 1.624 & -143.650 \\
\hline STORY6 & WIND & 0.000 & -6417.734 & 248.415 & 47087.490 \\
\hline STORY6 & WIND & 114.000 & -6417.734 & 248.415 & 18768.180 \\
\hline STORY6 & WIND & 228.000 & -6417.734 & 248.415 & -9551.133 \\
\hline STORY5 & DEAD & 0.000 & -2510.273 & 6.939 & 975.553 \\
\hline STORY5 & DEAD & 114.000 & -2474.650 & 6.939 & 184.521 \\
\hline STORY5 & DEAD & 228.000 & -2439.028 & 6.939 & -606.511 \\
\hline STORY5 & LIVE & 0.000 & -299.520 & 1.559 & 217.291 \\
\hline STORY5 & LIVE & 114.000 & -299.520 & 1.559 & 39.570 \\
\hline STORY5 & LIVE & 228.000 & -299.520 & 1.559 & -138.151 \\
\hline STORY5 & WIND & 0.000 & -6920.076 & 256.248 & 48195.830 \\
\hline STORY5 & WIND & 114.000 & -6920.076 & 256.248 & 18983.520 \\
\hline STORY5 & WIND & 228.000 & -6920.076 & 256.248 & -10228.790 \\
\hline STORY4 & DEAD & 0.000 & -2678.011 & 6.839 & 905.254 \\
\hline STORY4 & DEAD & 114.000 & -2637.480 & 6.839 & 125.647 \\
\hline STORY4 & DEAD & 228.000 & -2596.949 & 6.839 & -653.959 \\
\hline STORY4 & LIVE & 0.000 & -311.040 & 1.673 & 233.010 \\
\hline STORY4 & LIVE & 114.000 & -311.040 & 1.673 & 42.269 \\
\hline STORY4 & LIVE & 228.000 & -311.040 & 1.673 & -148.472 \\
\hline STORY4 & WIND & 0.000 & -7486.163 & 264.499 & 42595.510 \\
\hline STORY4 & WIND & 114.000 & -7486.163 & 264.499 & 12442.660 \\
\hline STORY4 & WIND & 228.000 & -7486.163 & 264.499 & -17710.200 \\
\hline STORY3 & DEAD & 0.000 & -2851.823 & 6.141 & 849.906 \\
\hline STORY3 & DEAD & 119.000 & -2804.061 & 6.141 & 119.121 \\
\hline STORY3 & DEAD & 238.000 & -2756.299 & 6.141 & -611.664 \\
\hline STORY3 & LIVE & 0.000 & -322.560 & 1.691 & 230.805 \\
\hline STORY3 & LIVE & 119.000 & -322.560 & 1.691 & 29.631 \\
\hline STORY3 & LIVE & 238.000 & -322.560 & 1.691 & -171.544 \\
\hline STORY3 & WIND & 0.000 & -7885.339 & 272.261 & 63358.860 \\
\hline STORY3 & WIND & 119.000 & -7885.339 & 272.261 & 30959.790 \\
\hline STORY3 & WIND & 238.000 & -7885.339 & 272.261 & -1439.280 \\
\hline STORY2 & DEAD & 0.000 & -3039.636 & 6.855 & 988.579 \\
\hline STORY2 & DEAD & 119.000 & -2986.089 & 6.855 & 172.879 \\
\hline STORY2 & DEAD & 238.000 & -2932.542 & 6.855 & -642.821 \\
\hline STORY2 & LIVE & 0.000 & -334.080 & 1.831 & 264.970 \\
\hline STORY2 & LIVE & 119.000 & -334.080 & 1.831 & 47.030 \\
\hline STORY2 & LIVE & 238.000 & -334.080 & 1.831 & -170.910 \\
\hline
\end{tabular}




\begin{tabular}{|c|c|c|c|c|c|}
\hline STORY2 & WIND & 0.000 & -8265.627 & 279.335 & 88946.340 \\
\hline STORY2 & WIND & 119.000 & -8265.627 & 279.335 & 55705.430 \\
\hline STORY2 & WIND & 238.000 & -8265.627 & 279.335 & 22464.530 \\
\hline STORY1 & DEAD & 0.000 & -3242.249 & 4.198 & 362.616 \\
\hline STORY1 & DEAD & 119.000 & -3182.587 & 4.198 & -136.975 \\
\hline STORY1 & DEAD & 238.000 & -3122.926 & 4.198 & -636.565 \\
\hline STORY1 & LIVE & 0.000 & -345.600 & 1.131 & 97.666 \\
\hline STORY1 & LIVE & 119.000 & -345.600 & 1.131 & -36.892 \\
\hline STORY1 & LIVE & 238.000 & -345.600 & 1.131 & -171.450 \\
\hline STORY1 & WIND & 0.000 & -8525.666 & 285.845 & 133777.300 \\
\hline STORY1 & WIND & 119.000 & -8525.666 & 285.845 & 99761.670 \\
\hline STORY1 & WIND & 238.000 & -8525.666 & 285.845 & 65746.100 \\
\hline STORY30 & DEAD & 0.000 & -66.326 & 0.669 & 95.505 \\
\hline STORY30 & DEAD & 119.000 & -62.979 & 0.669 & 15.887 \\
\hline STORY30 & DEAD & 238.000 & -59.633 & 0.669 & -63.731 \\
\hline STORY30 & LIVE & 0.000 & -11.520 & 0.180 & 25.657 \\
\hline STORY30 & LIVE & 119.000 & -11.520 & 0.180 & 4.268 \\
\hline STORY30 & LIVE & 238.000 & -11.520 & 0.180 & -17.121 \\
\hline STORY30 & WIND & 0.000 & -5.895 & 5.823 & 831.138 \\
\hline STORY30 & WIND & 119.000 & -5.895 & 5.823 & 138.192 \\
\hline STORY30 & WIND & 238.000 & -5.895 & 5.823 & -554.754 \\
\hline STORY29 & DEAD & 0.000 & -132.652 & 0.631 & 89.811 \\
\hline STORY29 & DEAD & 119.000 & -129.305 & 0.631 & 14.750 \\
\hline STORY29 & DEAD & 238.000 & -125.959 & 0.631 & -60.311 \\
\hline STORY29 & LIVE & 0.000 & -23.040 & 0.169 & 24.128 \\
\hline STORY29 & LIVE & 119.000 & -23.040 & 0.169 & 3.963 \\
\hline STORY29 & LIVE & 238.000 & -23.040 & 0.169 & -16.203 \\
\hline STORY29 & WIND & 0.000 & -29.134 & 17.401 & 2499.954 \\
\hline STORY29 & WIND & 119.000 & -29.134 & 17.401 & 429.189 \\
\hline STORY29 & WIND & 238.000 & -29.134 & 17.401 & -1641.577 \\
\hline STORY28 & DEAD & 0.000 & -200.878 & 1.423 & 202.660 \\
\hline STORY28 & DEAD & 119.000 & -196.746 & 1.423 & 33.371 \\
\hline STORY28 & DEAD & 238.000 & -192.614 & 1.423 & -135.919 \\
\hline STORY28 & LIVE & 0.000 & -34.560 & 0.382 & 54.445 \\
\hline STORY28 & LIVE & 119.000 & -34.560 & 0.382 & 8.965 \\
\hline STORY28 & LIVE & 238.000 & -34.560 & 0.382 & -36.515 \\
\hline STORY28 & WIND & 0.000 & -75.475 & 28.884 & 4149.901 \\
\hline STORY28 & WIND & 119.000 & -75.475 & 28.884 & 712.717 \\
\hline STORY28 & WIND & 238.000 & -75.475 & 28.884 & -2724.468 \\
\hline STORY27 & DEAD & 0.000 & -269.104 & 1.309 & 185.248 \\
\hline STORY27 & DEAD & 119.000 & -264.972 & 1.309 & 29.423 \\
\hline STORY27 & DEAD & 238.000 & -260.840 & 1.309 & -126.401 \\
\hline STORY27 & LIVE & 0.000 & -46.080 & 0.352 & 49.767 \\
\hline STORY27 & LIVE & 119.000 & -46.080 & 0.352 & 7.905 \\
\hline STORY 27 & LIVE & 238.000 & -46.080 & 0.352 & -33.958 \\
\hline STORY27 & WIND & 0.000 & -144.549 & 40.263 & 5804.564 \\
\hline STORY27 & WIND & 119.000 & -144.549 & 40.263 & 1013.271 \\
\hline
\end{tabular}




\begin{tabular}{|c|c|c|c|c|c|}
\hline STORY27 & WIND & 238.000 & -144.549 & 40.263 & -3778.022 \\
\hline STORY26 & DEAD & 0.000 & -341.729 & 2.436 & 344.711 \\
\hline STORY26 & DEAD & 119.000 & -335.779 & 2.436 & 54.868 \\
\hline STORY26 & DEAD & 238.000 & -329.830 & 2.436 & -234.976 \\
\hline STORY26 & LIVE & 0.000 & -57.600 & 0.654 & 92.607 \\
\hline STORY26 & LIVE & 119.000 & -57.600 & 0.654 & 14.740 \\
\hline STORY26 & LIVE & 238.000 & -57.600 & 0.654 & -63.126 \\
\hline STORY26 & WIND & 0.000 & -236.289 & 51.546 & 7446.715 \\
\hline STORY26 & WIND & 119.000 & -236.289 & 51.546 & 1312.757 \\
\hline STORY26 & WIND & 238.000 & -236.289 & 51.546 & -4821.201 \\
\hline STORY25 & DEAD & 0.000 & -414.355 & 2.245 & 320.026 \\
\hline STORY25 & DEAD & 119.000 & -408.405 & 2.245 & 52.836 \\
\hline STORY25 & DEAD & 238.000 & -402.455 & 2.245 & -214.354 \\
\hline STORY25 & LIVE & 0.000 & -69.120 & 0.603 & 85.975 \\
\hline STORY25 & LIVE & 119.000 & -69.120 & 0.603 & 14.194 \\
\hline STORY25 & LIVE & 238.000 & -69.120 & 0.603 & -57.586 \\
\hline STORY25 & WIND & 0.000 & -350.351 & 62.718 & 9093.874 \\
\hline STORY25 & WIND & 119.000 & -350.351 & 62.718 & 1630.487 \\
\hline STORY25 & WIND & 238.000 & -350.351 & 62.718 & -5832.899 \\
\hline STORY24 & DEAD & 0.000 & -489.480 & 2.901 & 413.326 \\
\hline STORY24 & DEAD & 119.000 & -482.497 & 2.901 & 68.145 \\
\hline STORY24 & DEAD & 238.000 & -475.515 & 2.901 & -277.036 \\
\hline STORY24 & LIVE & 0.000 & -80.640 & 0.779 & 111.040 \\
\hline STORY24 & LIVE & 119.000 & -80.640 & 0.779 & 18.307 \\
\hline STORY24 & LIVE & 238.000 & -80.640 & 0.779 & -74.426 \\
\hline STORY24 & WIND & 0.000 & -486.569 & 73.789 & 10741.810 \\
\hline STORY24 & WIND & 119.000 & -486.569 & 73.789 & 1960.957 \\
\hline STORY24 & WIND & 238.000 & -486.569 & 73.789 & -6819.891 \\
\hline STORY23 & DEAD & 0.000 & -564.605 & 2.728 & 388.667 \\
\hline STORY23 & DEAD & 119.000 & -557.623 & 2.728 & 64.068 \\
\hline STORY23 & DEAD & 238.000 & -550.640 & 2.728 & -260.531 \\
\hline STORY23 & LIVE & 0.000 & -92.160 & 0.733 & 104.416 \\
\hline STORY23 & LIVE & 119.000 & -92.160 & 0.733 & 17.212 \\
\hline STORY23 & LIVE & 238.000 & -92.160 & 0.733 & -69.992 \\
\hline STORY23 & WIND & 0.000 & -644.771 & 84.744 & 12382.580 \\
\hline STORY23 & WIND & 119.000 & -644.771 & 84.744 & 2298.047 \\
\hline STORY23 & WIND & 238.000 & -644.771 & 84.744 & -7786.491 \\
\hline STORY22 & DEAD & 0.000 & -642.431 & 3.286 & 460.528 \\
\hline STORY22 & DEAD & 119.000 & -634.333 & 3.286 & 69.484 \\
\hline STORY22 & DEAD & 238.000 & -626.234 & 3.286 & -321.560 \\
\hline STORY22 & LIVE & 0.000 & -103.680 & 0.883 & 123.721 \\
\hline STORY22 & LIVE & 119.000 & -103.680 & 0.883 & 18.667 \\
\hline STORY 22 & LIVE & 238.000 & -103.680 & 0.883 & -86.387 \\
\hline STORY22 & WIND & 0.000 & -824.576 & 95.598 & 14043.190 \\
\hline STORY22 & WIND & 119.000 & -824.576 & 95.598 & 2667.040 \\
\hline STORY22 & WIND & 238.000 & -824.576 & 95.598 & -8709.107 \\
\hline STORY21 & DEAD & 0.000 & -723.156 & 3.852 & 548.468 \\
\hline
\end{tabular}




\begin{tabular}{|c|c|c|c|c|c|}
\hline STORY21 & DEAD & 119.000 & -713.859 & 3.852 & 90.122 \\
\hline STORY21 & DEAD & 238.000 & -704.563 & 3.852 & -368.223 \\
\hline STORY21 & LIVE & 0.000 & -115.200 & 1.035 & 147.346 \\
\hline STORY21 & LIVE & 119.000 & -115.200 & 1.035 & 24.211 \\
\hline STORY21 & LIVE & 238.000 & -115.200 & 1.035 & -98.923 \\
\hline STORY21 & WIND & 0.000 & -1025.813 & 106.343 & 15719.540 \\
\hline STORY21 & WIND & 119.000 & -1025.813 & 106.343 & 3064.754 \\
\hline STORY21 & WIND & 238.000 & -1025.813 & 106.343 & -9590.030 \\
\hline STORY20 & DEAD & 0.000 & -803.881 & 3.626 & 517.070 \\
\hline STORY20 & DEAD & 119.000 & -794.584 & 3.626 & 85.621 \\
\hline STORY20 & DEAD & 238.000 & -785.288 & 3.626 & -345.828 \\
\hline STORY20 & LIVE & 0.000 & -126.720 & 0.974 & 138.911 \\
\hline STORY20 & LIVE & 119.000 & -126.720 & 0.974 & 23.002 \\
\hline STORY20 & LIVE & 238.000 & -126.720 & 0.974 & -92.907 \\
\hline STORY20 & WIND & 0.000 & -1248.519 & 116.956 & 17368.910 \\
\hline STORY20 & WIND & 119.000 & -1248.519 & 116.956 & 3451.119 \\
\hline STORY20 & WIND & 238.000 & -1248.519 & 116.956 & -10466.680 \\
\hline STORY 19 & DEAD & 0.000 & -887.705 & 3.996 & 548.259 \\
\hline STORY19 & DEAD & 119.000 & -877.128 & 3.996 & 72.712 \\
\hline STORY19 & DEAD & 238.000 & -866.551 & 3.996 & -402.835 \\
\hline STORY19 & LIVE & 0.000 & -138.240 & 1.074 & 147.290 \\
\hline STORY 19 & LIVE & 119.000 & -138.240 & 1.074 & 19.534 \\
\hline STORY19 & LIVE & 238.000 & -138.240 & 1.074 & -108.222 \\
\hline STORY19 & WIND & 0.000 & -1491.870 & 127.471 & 19084.380 \\
\hline STORY19 & WIND & 119.000 & -1491.870 & 127.471 & 3915.320 \\
\hline STORY19 & WIND & 238.000 & -1491.870 & 127.471 & -11253.750 \\
\hline STORY18 & DEAD & 0.000 & -978.330 & 5.132 & 721.730 \\
\hline STORY18 & DEAD & 119.000 & -964.943 & 5.132 & 111.070 \\
\hline STORY18 & DEAD & 238.000 & -951.557 & 5.132 & -499.590 \\
\hline STORY18 & LIVE & 0.000 & -149.760 & 1.379 & 193.893 \\
\hline STORY18 & LIVE & 119.000 & -149.760 & 1.379 & 29.839 \\
\hline STORY18 & LIVE & 238.000 & -149.760 & 1.379 & -134.215 \\
\hline STORY18 & WIND & 0.000 & -1755.459 & 137.911 & 20912.310 \\
\hline STORY18 & WIND & 119.000 & -1755.459 & 137.911 & 4500.916 \\
\hline STORY18 & WIND & 238.000 & -1755.459 & 137.911 & -11910.480 \\
\hline STORY17 & DEAD & 0.000 & -1068.954 & 4.428 & 619.603 \\
\hline STORY17 & DEAD & 119.000 & -1055.568 & 4.428 & 92.645 \\
\hline STORY 17 & DEAD & 238.000 & -1042.181 & 4.428 & -434.314 \\
\hline STORY 17 & LIVE & 0.000 & -161.280 & 1.190 & 166.457 \\
\hline STORY 17 & LIVE & 119.000 & -161.280 & 1.190 & 24.889 \\
\hline STORY 17 & LIVE & 238.000 & -161.280 & 1.190 & -116.679 \\
\hline STORY17 & WIND & 0.000 & -2040.427 & 148.149 & 22629.760 \\
\hline STORY17 & WIND & 119.000 & -2040.427 & 148.149 & 4999.994 \\
\hline STORY17 & WIND & 238.000 & -2040.427 & 148.149 & -12629.770 \\
\hline STORY16 & DEAD & 0.000 & -1167.178 & 5.608 & 780.697 \\
\hline STORY16 & DEAD & 119.000 & -1150.651 & 5.608 & 113.331 \\
\hline STORY16 & DEAD & 238.000 & -1134.125 & 5.608 & -554.036 \\
\hline
\end{tabular}




\begin{tabular}{|c|c|c|c|c|c|}
\hline STORY16 & LIVE & 0.000 & -172.800 & 1.507 & 209.735 \\
\hline STORY16 & LIVE & 119.000 & -172.800 & 1.507 & 30.446 \\
\hline STORY16 & LIVE & 238.000 & -172.800 & 1.507 & -148.842 \\
\hline STORY16 & WIND & 0.000 & -2344.466 & 158.384 & 24576.880 \\
\hline STORY16 & WIND & 119.000 & -2344.466 & 158.384 & 5729.174 \\
\hline STORY16 & WIND & 238.000 & -2344.466 & 158.384 & -13118.530 \\
\hline STORY15 & DEAD & 0.000 & -1265.402 & 5.259 & 766.485 \\
\hline STORY15 & DEAD & 119.000 & -1248.875 & 5.259 & 140.661 \\
\hline STORY15 & DEAD & 238.000 & -1232.349 & 5.259 & -485.164 \\
\hline STORY15 & LIVE & 0.000 & -184.320 & 1.413 & 205.917 \\
\hline STORY 15 & LIVE & 119.000 & -184.320 & 1.413 & 37.789 \\
\hline STORY15 & LIVE & 238.000 & -184.320 & 1.413 & -130.340 \\
\hline STORY15 & WIND & 0.000 & -2669.967 & 168.391 & 26349.060 \\
\hline STORY15 & WIND & 119.000 & -2669.967 & 168.391 & 6310.500 \\
\hline STORY15 & WIND & 238.000 & -2669.967 & 168.391 & -13728.060 \\
\hline STORY14 & DEAD & 0.000 & -1363.626 & 4.941 & 686.142 \\
\hline STORY14 & DEAD & 119.000 & -1347.099 & 4.941 & 98.202 \\
\hline STORY14 & DEAD & 238.000 & -1330.572 & 4.941 & -489.738 \\
\hline STORY14 & LIVE & 0.000 & -195.840 & 1.327 & 184.333 \\
\hline STORY14 & LIVE & 119.000 & -195.840 & 1.327 & 26.382 \\
\hline STORY14 & LIVE & 238.000 & -195.840 & 1.327 & -131.569 \\
\hline STORY 14 & WIND & 0.000 & -3015.326 & 178.260 & 28136.390 \\
\hline STORY14 & WIND & 119.000 & -3015.326 & 178.260 & 6923.416 \\
\hline STORY14 & WIND & 238.000 & -3015.326 & 178.260 & -14289.560 \\
\hline STORY13 & DEAD & 0.000 & -1470.249 & 6.016 & 845.007 \\
\hline STORY13 & DEAD & 119.000 & -1450.252 & 6.016 & 129.125 \\
\hline STORY13 & DEAD & 238.000 & -1430.255 & 6.016 & -586.757 \\
\hline STORY13 & LIVE & 0.000 & -207.360 & 1.616 & 227.014 \\
\hline STORY13 & LIVE & 119.000 & -207.360 & 1.616 & 34.690 \\
\hline STORY13 & LIVE & 238.000 & -207.360 & 1.616 & -157.633 \\
\hline STORY13 & WIND & 0.000 & -3378.294 & 188.212 & 30313.870 \\
\hline STORY13 & WIND & 119.000 & -3378.294 & 188.212 & 7916.615 \\
\hline STORY13 & WIND & 238.000 & -3378.294 & 188.212 & -14480.640 \\
\hline STORY12 & DEAD & 0.000 & -1576.873 & 5.198 & 727.843 \\
\hline STORY 12 & DEAD & 119.000 & -1556.875 & 5.198 & 109.309 \\
\hline STORY12 & DEAD & 238.000 & -1536.878 & 5.198 & -509.225 \\
\hline STORY12 & LIVE & 0.000 & -218.880 & 1.396 & 195.520 \\
\hline STORY12 & LIVE & 119.000 & -218.880 & 1.396 & 29.359 \\
\hline STORY12 & LIVE & 238.000 & -218.880 & 1.396 & -136.802 \\
\hline STORY12 & WIND & 0.000 & -3762.442 & 197.810 & 32271.510 \\
\hline STORY12 & WIND & 119.000 & -3762.442 & 197.810 & 8732.102 \\
\hline STORY12 & WIND & 238.000 & -3762.442 & 197.810 & -14807.300 \\
\hline STORY11 & DEAD & 0.000 & -1692.696 & 6.267 & 879.442 \\
\hline STORY11 & DEAD & 119.000 & -1668.898 & 6.267 & 133.672 \\
\hline STORY11 & DEAD & 238.000 & -1645.099 & 6.267 & -612.099 \\
\hline STORY11 & LIVE & 0.000 & -230.400 & 1.684 & 236.385 \\
\hline STORY11 & LIVE & 119.000 & -230.400 & 1.684 & 35.966 \\
\hline
\end{tabular}




\begin{tabular}{|c|c|c|c|c|c|}
\hline STORY11 & LIVE & 238.000 & -230.400 & 1.684 & -164.453 \\
\hline STORY11 & WIND & 0.000 & -4162.534 & 207.207 & 34708.120 \\
\hline STORY11 & WIND & 119.000 & -4162.534 & 207.207 & 10050.480 \\
\hline STORY11 & WIND & 238.000 & -4162.534 & 207.207 & -14607.170 \\
\hline STORY10 & DEAD & 0.000 & -1808.523 & 5.459 & 767.122 \\
\hline STORY10 & DEAD & 119.000 & -1784.725 & 5.459 & 117.556 \\
\hline STORY10 & DEAD & 238.000 & -1760.926 & 5.459 & -532.009 \\
\hline STORY10 & LIVE & 0.000 & -241.922 & 1.463 & 205.358 \\
\hline STORY10 & LIVE & 119.000 & -241.922 & 1.463 & 31.253 \\
\hline STORY10 & LIVE & 238.000 & -241.922 & 1.463 & -142.853 \\
\hline STORY10 & WIND & 0.000 & -4580.825 & 218.525 & 37895.390 \\
\hline STORY10 & WIND & 119.000 & -4580.825 & 218.525 & 11890.970 \\
\hline STORY10 & WIND & 238.000 & -4580.825 & 218.525 & -14113.440 \\
\hline STORY9 & DEAD & 0.000 & -1934.368 & 6.316 & 877.484 \\
\hline STORY9 & DEAD & 119.000 & -1906.438 & 6.316 & 125.844 \\
\hline STORY9 & DEAD & 238.000 & -1878.508 & 6.316 & -625.796 \\
\hline STORY9 & LIVE & 0.000 & -253.450 & 1.718 & 240.505 \\
\hline STORY9 & LIVE & 119.000 & -253.450 & 1.718 & 36.039 \\
\hline STORY9 & LIVE & 238.000 & -253.450 & 1.718 & -168.428 \\
\hline STORY9 & WIND & 0.000 & -4984.345 & 207.791 & 39178.700 \\
\hline STORY9 & WIND & 119.000 & -4984.345 & 207.791 & 14451.610 \\
\hline STORY9 & WIND & 238.000 & -4984.345 & 207.791 & -10275.480 \\
\hline STORY8 & DEAD & 0.000 & -2060.174 & 6.091 & 897.416 \\
\hline STORY8 & DEAD & 119.000 & -2032.243 & 6.091 & 172.540 \\
\hline STORY8 & DEAD & 238.000 & -2004.313 & 6.091 & -552.338 \\
\hline STORY 8 & LIVE & 0.000 & -264.950 & 1.505 & 212.536 \\
\hline STORY8 & LIVE & 119.000 & -264.950 & 1.505 & 33.384 \\
\hline STORY8 & LIVE & 238.000 & -264.950 & 1.505 & -145.768 \\
\hline STORY8 & WIND & 0.000 & -5354.556 & 216.203 & 46166.760 \\
\hline STORY8 & WIND & 119.000 & -5354.556 & 216.203 & 20438.560 \\
\hline STORY8 & WIND & 238.000 & -5354.556 & 216.203 & -5289.639 \\
\hline STORY7 & DEAD & 0.000 & -2198.444 & 6.676 & 918.653 \\
\hline STORY7 & DEAD & 114.000 & -2169.589 & 6.676 & 157.631 \\
\hline STORY7 & DEAD & 228.000 & -2140.735 & 6.676 & -603.392 \\
\hline STORY7 & LIVE & 0.000 & -276.478 & 1.495 & 207.356 \\
\hline STORY7 & LIVE & 114.000 & -276.478 & 1.495 & 36.907 \\
\hline STORY7 & LIVE & 228.000 & -276.478 & 1.495 & -133.542 \\
\hline STORY7 & WIND & 0.000 & -5848.986 & 246.001 & 45556.540 \\
\hline STORY7 & WIND & 114.000 & -5848.986 & 246.001 & 17512.460 \\
\hline STORY7 & WIND & 228.000 & -5848.986 & 246.001 & -10531.610 \\
\hline STORY6 & DEAD & 0.000 & -2347.899 & 7.099 & 989.984 \\
\hline STORY6 & DEAD & 114.000 & -2314.612 & 7.099 & 180.664 \\
\hline STORY6 & DEAD & 228.000 & -2281.325 & 7.099 & -628.656 \\
\hline STORY6 & LIVE & 0.000 & -287.999 & 1.626 & 226.728 \\
\hline STORY6 & LIVE & 114.000 & -287.999 & 1.626 & 41.348 \\
\hline STORY6 & LIVE & 228.000 & -287.999 & 1.626 & -144.031 \\
\hline STORY6 & WIND & 0.000 & -6340.711 & 250.866 & 47494.000 \\
\hline
\end{tabular}




\begin{tabular}{|c|c|c|c|c|c|}
\hline STORY6 & WIND & 114.000 & -6340.711 & 250.866 & 18895.230 \\
\hline STORY6 & WIND & 228.000 & -6340.711 & 250.866 & -9703.536 \\
\hline STORY5 & DEAD & 0.000 & -2503.251 & 6.943 & 975.809 \\
\hline STORY5 & DEAD & 114.000 & -2467.628 & 6.943 & 184.349 \\
\hline STORY5 & DEAD & 228.000 & -2432.005 & 6.943 & -607.112 \\
\hline STORY5 & LIVE & 0.000 & -299.520 & 1.558 & 217.258 \\
\hline STORY5 & LIVE & 114.000 & -299.520 & 1.558 & 39.594 \\
\hline STORY5 & LIVE & 228.000 & -299.520 & 1.558 & -138.070 \\
\hline STORY5 & WIND & 0.000 & -6854.013 & 259.026 & 48532.080 \\
\hline STORY5 & WIND & 114.000 & -6854.013 & 259.026 & 19003.160 \\
\hline STORY5 & WIND & 228.000 & -6854.013 & 259.026 & -10525.760 \\
\hline STORY4 & DEAD & 0.000 & -2671.002 & 6.838 & 905.216 \\
\hline STORY4 & DEAD & 114.000 & -2630.471 & 6.838 & 125.692 \\
\hline STORY4 & DEAD & 228.000 & -2589.940 & 6.838 & -653.832 \\
\hline STORY4 & LIVE & 0.000 & -311.040 & 1.673 & 233.017 \\
\hline STORY4 & LIVE & 114.000 & -311.040 & 1.673 & 42.263 \\
\hline STORY4 & LIVE & 228.000 & -311.040 & 1.673 & -148.491 \\
\hline STORY4 & WIND & 0.000 & -7428.333 & 266.166 & 42745.370 \\
\hline STORY4 & WIND & 114.000 & -7428.333 & 266.166 & 12402.430 \\
\hline STORY4 & WIND & 228.000 & -7428.333 & 266.166 & -17940.500 \\
\hline STORY3 & DEAD & 0.000 & -2844.827 & 6.141 & 849.931 \\
\hline STORY3 & DEAD & 119.000 & -2797.065 & 6.141 & 119.111 \\
\hline STORY3 & DEAD & 238.000 & -2749.303 & 6.141 & -611.709 \\
\hline STORY3 & LIVE & 0.000 & -322.560 & 1.691 & 230.803 \\
\hline STORY3 & LIVE & 119.000 & -322.560 & 1.691 & 29.632 \\
\hline STORY3 & LIVE & 238.000 & -322.560 & 1.691 & -171.539 \\
\hline STORY3 & WIND & 0.000 & -7831.847 & 273.092 & 63456.180 \\
\hline STORY3 & WIND & 119.000 & -7831.847 & 273.092 & 30958.220 \\
\hline STORY3 & WIND & 238.000 & -7831.847 & 273.092 & -1539.748 \\
\hline STORY2 & DEAD & 0.000 & -3032.651 & 6.855 & 988.587 \\
\hline STORY2 & DEAD & 119.000 & -2979.105 & 6.855 & 172.883 \\
\hline STORY2 & DEAD & 238.000 & -2925.558 & 6.855 & -642.821 \\
\hline STORY2 & LIVE & 0.000 & -334.080 & 1.831 & 264.970 \\
\hline STORY2 & LIVE & 119.000 & -334.080 & 1.831 & 47.030 \\
\hline STORY2 & LIVE & 238.000 & -334.080 & 1.831 & -170.911 \\
\hline STORY2 & WIND & 0.000 & -8214.807 & 279.838 & 89002.780 \\
\hline STORY2 & WIND & 119.000 & -8214.807 & 279.838 & 55702.120 \\
\hline STORY2 & WIND & 238.000 & -8214.807 & 279.838 & 22401.460 \\
\hline STORY1 & DEAD & 0.000 & -3235.273 & 4.198 & 362.620 \\
\hline STORY1 & DEAD & 119.000 & -3175.611 & 4.198 & -136.976 \\
\hline STORY1 & DEAD & 238.000 & -3115.950 & 4.198 & -636.573 \\
\hline STORY1 & LIVE & 0.000 & -345.600 & 1.131 & 97.666 \\
\hline STORY1 & LIVE & 119.000 & -345.600 & 1.131 & -36.892 \\
\hline STORY1 & LIVE & 238.000 & -345.600 & 1.131 & -171.450 \\
\hline STORY1 & WIND & 0.000 & -8476.116 & 286.004 & 133790.400 \\
\hline STORY1 & WIND & 119.000 & -8476.116 & 286.004 & 99755.880 \\
\hline STORY1 & WIND & 238.000 & -8476.116 & 286.004 & 65721.380 \\
\hline
\end{tabular}




\begin{tabular}{|c|c|c|c|c|c|}
\hline \\
\hline & & & & & \\
\hline & & & & & \\
\hline & & & & & \\
\hline & & & & & \\
\hline & & & & & \\
\hline & & & & & \\
\hline & & & & & \\
\hline & & & & & \\
\hline & & & & & \\
\hline & & & & & \\
\hline & & & & & \\
\hline & & & & & \\
\hline & & & & & \\
\hline & & & & & \\
\hline & & & & & \\
\hline & & & & & \\
\hline & & & & & \\
\hline & & & & & \\
\hline & & & & & \\
\hline Story & Beam & Load & Loc & $\mathrm{V} 2$ & M3 \\
\hline STORY30 & $\mathrm{B} 1$ & DEAD & 9.000 & -27.014 & 435.544 \\
\hline STORY30 & $\mathrm{B} 1$ & DEAD & 30.000 & -24.554 & 977.009 \\
\hline STORY30 & $\mathrm{B} 1$ & DEAD & 51.000 & -22.093 & 1466.799 \\
\hline STORY30 & B1 & DEAD & 72.000 & -19.632 & 1904.911 \\
\hline STORY30 & $\mathrm{B} 1$ & DEAD & 72.000 & -13.207 & 1920.671 \\
\hline STORY30 & $\mathrm{B} 1$ & DEAD & 96.000 & -10.395 & 2203.894 \\
\hline STORY30 & $\mathrm{B} 1$ & DEAD & 120.000 & -7.583 & 2419.622 \\
\hline STORY30 & $\mathrm{B} 1$ & DEAD & 144.000 & -4.770 & 2567.854 \\
\hline STORY30 & $\mathrm{B} 1$ & DEAD & 144.000 & 4.770 & 2567.854 \\
\hline STORY30 & $\mathrm{B} 1$ & DEAD & 168.000 & 7.583 & 2419.622 \\
\hline STORY30 & $\mathrm{B} 1$ & DEAD & 192.000 & 10.395 & 2203.894 \\
\hline STORY30 & $\mathrm{B} 1$ & DEAD & 216.000 & 13.207 & 1920.671 \\
\hline STORY30 & $\mathrm{B} 1$ & DEAD & 216.000 & 19.632 & 1904.911 \\
\hline STORY30 & $\mathrm{B} 1$ & DEAD & 237.000 & 22.093 & 1466.799 \\
\hline STORY30 & $\mathrm{B} 1$ & DEAD & 258.000 & 24.554 & 977.009 \\
\hline STORY30 & $\mathrm{B} 1$ & DEAD & 279.000 & 27.014 & 435.544 \\
\hline STORY30 & $\mathrm{B} 1$ & LIVE & 9.000 & -5.427 & 166.905 \\
\hline STORY30 & $\mathrm{B} 1$ & LIVE & 30.000 & -5.427 & 280.877 \\
\hline STORY30 & $\mathrm{B} 1$ & LIVE & 51.000 & -5.427 & 394.849 \\
\hline STORY30 & $\mathrm{B} 1$ & LIVE & 72.000 & -5.427 & 508.820 \\
\hline STORY30 & $\mathrm{B} 1$ & LIVE & 72.000 & -2.336 & 513.671 \\
\hline STORY 30 & B1 & LIVE & 96.000 & -2.336 & 569.736 \\
\hline STORY30 & B1 & LIVE & 120.000 & -2.336 & 625.802 \\
\hline STORY30 & $\mathrm{B} 1$ & LIVE & 144.000 & -2.336 & 681.867 \\
\hline STORY30 & $\mathrm{B} 1$ & LIVE & 144.000 & 2.336 & 681.867 \\
\hline STORY30 & $\mathrm{B} 1$ & LIVE & 168.000 & 2.336 & 625.802 \\
\hline STORY30 & $\mathrm{B} 1$ & LIVE & 192.000 & 2.336 & 569.736 \\
\hline
\end{tabular}




\begin{tabular}{|c|c|c|c|c|c|}
\hline STORY30 & $\mathrm{B} 1$ & LIVE & 216.000 & 2.336 & 513.671 \\
\hline STORY30 & $\mathrm{B} 1$ & LIVE & 216.000 & 5.427 & 508.820 \\
\hline STORY30 & B1 & LIVE & 237.000 & 5.427 & 394.849 \\
\hline STORY30 & B1 & LIVE & 258.000 & 5.427 & 280.877 \\
\hline STORY30 & $\mathrm{B} 1$ & LIVE & 279.000 & 5.427 & 166.905 \\
\hline STORY30 & $\mathrm{B} 1$ & WIND & 9.000 & 5.572 & 756.275 \\
\hline STORY30 & B1 & WIND & 30.000 & 5.572 & 639.256 \\
\hline STORY30 & B1 & WIND & 51.000 & 5.572 & 522.237 \\
\hline STORY30 & $\mathrm{B} 1$ & WIND & 72.000 & 5.572 & 405.218 \\
\hline STORY30 & $\mathrm{B} 1$ & WIND & 72.000 & 5.549 & 401.399 \\
\hline STORY30 & $\mathrm{B} 1$ & WIND & 96.000 & 5.549 & 268.232 \\
\hline STORY30 & $\mathrm{B} 1$ & WIND & 120.000 & 5.549 & 135.066 \\
\hline STORY30 & $\mathrm{B} 1$ & WIND & 144.000 & 5.549 & 1.899 \\
\hline STORY30 & $\mathrm{B} 1$ & WIND & 144.000 & 5.549 & -1.899 \\
\hline STORY30 & $\mathrm{B} 1$ & WIND & 168.000 & 5.549 & -135.066 \\
\hline STORY30 & B1 & WIND & 192.000 & 5.549 & -268.232 \\
\hline STORY30 & $\mathrm{B} 1$ & WIND & 216.000 & 5.549 & -401.399 \\
\hline STORY30 & $\mathrm{B} 1$ & WIND & 216.000 & 5.572 & -405.218 \\
\hline STORY30 & $\mathrm{B} 1$ & WIND & 237.000 & 5.572 & -522.237 \\
\hline STORY30 & B1 & WIND & 258.000 & 5.572 & -639.256 \\
\hline STORY30 & B1 & WIND & 279.000 & 5.572 & -756.275 \\
\hline STORY29 & $\mathrm{B} 1$ & DEAD & 9.000 & -27.016 & 350.007 \\
\hline STORY29 & B1 & DEAD & 30.000 & -24.556 & 891.511 \\
\hline STORY29 & B1 & DEAD & 51.000 & -22.095 & 1381.339 \\
\hline STORY29 & $\mathrm{B} 1$ & DEAD & 72.000 & -19.634 & 1819.490 \\
\hline STORY29 & B1 & DEAD & 72.000 & -13.207 & 1835.248 \\
\hline STORY29 & B1 & DEAD & 96.000 & -10.395 & 2118.475 \\
\hline STORY29 & B1 & DEAD & 120.000 & -7.583 & 2334.206 \\
\hline STORY29 & B1 & DEAD & 144.000 & -4.770 & 2482.441 \\
\hline STORY29 & $\mathrm{B} 1$ & DEAD & 144.000 & 4.770 & 2482.441 \\
\hline STORY29 & B1 & DEAD & 168.000 & 7.583 & 2334.206 \\
\hline STORY29 & $\mathrm{B} 1$ & DEAD & 192.000 & 10.395 & 2118.475 \\
\hline STORY29 & $\mathrm{B} 1$ & DEAD & 216.000 & 13.207 & 1835.248 \\
\hline STORY29 & B1 & DEAD & 216.000 & 19.634 & 1819.490 \\
\hline STORY29 & $\mathrm{B} 1$ & DEAD & 237.000 & 22.095 & 1381.339 \\
\hline STORY29 & B1 & DEAD & 258.000 & 24.556 & 891.511 \\
\hline STORY29 & B1 & DEAD & 279.000 & 27.016 & 350.007 \\
\hline STORY29 & B1 & LIVE & 9.000 & -5.428 & 143.926 \\
\hline STORY29 & $\mathrm{B} 1$ & LIVE & 30.000 & -5.428 & 257.908 \\
\hline STORY29 & B1 & LIVE & 51.000 & -5.428 & 371.890 \\
\hline STORY29 & B1 & LIVE & 72.000 & -5.428 & 485.872 \\
\hline STORY29 & B1 & LIVE & 72.000 & -2.336 & 490.722 \\
\hline STORY29 & $\mathrm{B} 1$ & LIVE & 96.000 & -2.336 & 546.788 \\
\hline STORY29 & B1 & LIVE & 120.000 & -2.336 & 602.855 \\
\hline STORY29 & B1 & LIVE & 144.000 & -2.336 & 658.921 \\
\hline STORY29 & B1 & LIVE & 144.000 & 2.336 & 658.921 \\
\hline STORY29 & $\mathrm{B} 1$ & LIVE & 168.000 & 2.336 & 602.855 \\
\hline
\end{tabular}




\begin{tabular}{|c|c|c|c|c|c|}
\hline STORY29 & $\mathrm{B} 1$ & LIVE & 192.000 & 2.336 & 546.788 \\
\hline STORY29 & $\mathrm{B} 1$ & LIVE & 216.000 & 2.336 & 490.722 \\
\hline STORY29 & $\mathrm{B} 1$ & LIVE & 216.000 & 5.428 & 485.872 \\
\hline STORY29 & $\mathrm{B} 1$ & LIVE & 237.000 & 5.428 & 371.890 \\
\hline STORY29 & $\mathrm{B} 1$ & LIVE & 258.000 & 5.428 & 257.908 \\
\hline STORY29 & $\mathrm{B} 1$ & LIVE & 279.000 & 5.428 & 143.926 \\
\hline STORY29 & $\mathrm{B} 1$ & WIND & 9.000 & 22.050 & 2992.573 \\
\hline STORY29 & $\mathrm{B} 1$ & WIND & 30.000 & 22.050 & 2529.521 \\
\hline STORY29 & $\mathrm{B} 1$ & WIND & 51.000 & 22.050 & 2066.470 \\
\hline STORY29 & $\mathrm{B} 1$ & WIND & 72.000 & 22.050 & 1603.419 \\
\hline STORY29 & $\mathrm{B} 1$ & WIND & 72.000 & 21.955 & 1588.306 \\
\hline STORY29 & $\mathrm{B} 1$ & WIND & 96.000 & 21.955 & 1061.376 \\
\hline STORY29 & $\mathrm{B} 1$ & WIND & 120.000 & 21.955 & 534.445 \\
\hline STORY29 & $\mathrm{Bl}$ & WIND & 144.000 & 21.955 & 7.515 \\
\hline STORY29 & $\mathrm{Bl}$ & WIND & 144.000 & 21.955 & -7.515 \\
\hline STORY29 & $\mathrm{Bl}$ & WIND & 168.000 & 21.955 & -534.445 \\
\hline STORY29 & $\mathrm{B} 1$ & WIND & 192.000 & 21.955 & -1061.376 \\
\hline STORY29 & $\mathrm{Bl}$ & WIND & 216.000 & 21.955 & -1588.306 \\
\hline STORY29 & $\mathrm{B} 1$ & WIND & 216.000 & 22.050 & -1603.419 \\
\hline STORY29 & $\mathrm{B} 1$ & WIND & 237.000 & 22.050 & -2066.470 \\
\hline STORY29 & $\mathrm{B} 1$ & WIND & 258.000 & 22.050 & -2529.521 \\
\hline STORY29 & $\mathrm{B} 1$ & WIND & 279.000 & 22.050 & -2992.573 \\
\hline STORY28 & $\mathrm{B} 1$ & DEAD & 10.000 & -26.901 & 273.090 \\
\hline STORY28 & $\mathrm{B} 1$ & DEAD & 30.667 & -24.480 & 804.026 \\
\hline STORY28 & $\mathrm{B} 1$ & DEAD & 51.333 & -22.058 & 1284.914 \\
\hline STORY28 & $\mathrm{B} 1$ & DEAD & 72.000 & -19.636 & 1715.753 \\
\hline STORY 28 & $\mathrm{~B} 1$ & DEAD & 72.000 & -13.207 & 1731.509 \\
\hline STORY28 & $\mathrm{B} 1$ & DEAD & 96.000 & -10.395 & 2014.739 \\
\hline STORY28 & $\mathrm{B} 1$ & DEAD & 120.000 & $\begin{array}{l}-7.583 \\
\end{array}$ & 2230.474 \\
\hline STORY28 & $\mathrm{B} 1$ & DEAD & 144.000 & -4.770 & 2378.713 \\
\hline STORY28 & $\mathrm{B} 1$ & DEAD & 144.000 & 4.770 & 2378.713 \\
\hline STORY28 & $\mathrm{B} 1$ & DEAD & 168.000 & 7.583 & 2230.474 \\
\hline STORY28 & $\mathrm{B} 1$ & DEAD & 192.000 & 10.395 & 2014.739 \\
\hline STORY28 & $\mathrm{B} 1$ & DEAD & 216.000 & 13.207 & 1731.509 \\
\hline STORY28 & $\mathrm{B} 1$ & DEAD & 216.000 & 19.636 & 1715.753 \\
\hline STORY28 & B1 & DEAD & 236.667 & 22.058 & 1284.914 \\
\hline STORY28 & $\mathrm{B} 1$ & DEAD & 257.333 & 24.480 & 804.026 \\
\hline STORY28 & $\mathrm{Bl}$ & DEAD & 278.000 & 26.901 & 273.090 \\
\hline STORY28 & $\mathrm{B} 1$ & LIVE & 10.000 & -5.428 & 121.447 \\
\hline STORY28 & $\mathrm{B} 1$ & LIVE & 30.667 & -5.428 & 233.633 \\
\hline STORY28 & $\mathrm{B} 1$ & LIVE & 51.333 & -5.428 & 345.818 \\
\hline STORY 28 & $\mathrm{~B} 1$ & LIVE & 72.000 & -5.428 & 458.003 \\
\hline STORY28 & $\mathrm{B} 1$ & LIVE & 72.000 & -2.336 & 462.852 \\
\hline STORY28 & $\mathrm{B} 1$ & LIVE & 96.000 & -2.336 & 518.920 \\
\hline STORY28 & $\mathrm{B} 1$ & LIVE & 120.000 & -2.336 & 574.987 \\
\hline STORY28 & $\mathrm{B} 1$ & LIVE & 144.000 & -2.336 & 631.054 \\
\hline STORY28 & $\mathrm{B} 1$ & LIVE & 144.000 & 2.336 & 631.054 \\
\hline
\end{tabular}




\begin{tabular}{|c|c|c|c|c|c|}
\hline STORY28 & $\mathrm{B} 1$ & LIVE & 168.000 & 2.336 & 574.987 \\
\hline STORY28 & $\mathrm{B} 1$ & LIVE & 192.000 & 2.336 & 518.920 \\
\hline STORY28 & $\mathrm{B} 1$ & LIVE & 216.000 & 2.336 & 462.852 \\
\hline STORY28 & $\mathrm{B} 1$ & LIVE & 216.000 & 5.428 & 458.003 \\
\hline STORY28 & $\mathrm{B} 1$ & LIVE & 236.667 & 5.428 & 345.818 \\
\hline STORY28 & $\mathrm{B} 1$ & LIVE & 257.333 & 5.428 & 233.633 \\
\hline STORY28 & $\mathrm{B} 1$ & LIVE & 278.000 & 5.428 & 121.447 \\
\hline STORY28 & $\mathrm{B} 1$ & WIND & 10.000 & 43.994 & 5926.786 \\
\hline STORY28 & $\mathrm{B} 1$ & WIND & 30.667 & 43.994 & 5017.587 \\
\hline STORY28 & $\mathrm{B} 1$ & WIND & 51.333 & 43.994 & 4108.387 \\
\hline STORY28 & $\mathrm{B} 1$ & WIND & 72.000 & 43.994 & 3199.188 \\
\hline STORY28 & $\mathrm{B} 1$ & WIND & 72.000 & 43.806 & 3169.032 \\
\hline STORY28 & $\mathrm{B} 1$ & WIND & 96.000 & 43.806 & 2117.686 \\
\hline STORY28 & $\mathrm{B} 1$ & WIND & 120.000 & 43.806 & 1066.340 \\
\hline STORY28 & $\mathrm{B} 1$ & WIND & 144.000 & 43.806 & 14.994 \\
\hline STORY28 & $\mathrm{B} 1$ & WIND & 144.000 & 43.806 & -14.994 \\
\hline STORY28 & $\mathrm{B} 1$ & WIND & 168.000 & 43.806 & -1066.340 \\
\hline STORY28 & $\mathrm{B} 1$ & WIND & 192.000 & 43.806 & -2117.686 \\
\hline STORY28 & B1 & WIND & 216.000 & 43.806 & -3169.032 \\
\hline STORY28 & $\mathrm{B} 1$ & WIND & 216.000 & 43.994 & -3199.188 \\
\hline STORY28 & $\mathrm{B} 1$ & WIND & 236.667 & 43.994 & -4108.387 \\
\hline STORY28 & $\mathrm{B} 1$ & WIND & 257.333 & 43.994 & -5017.587 \\
\hline STORY28 & $\mathrm{B} 1$ & WIND & 278.000 & 43.994 & -5926.786 \\
\hline STORY27 & $\mathrm{B} 1$ & DEAD & 10.000 & -26.903 & 180.437 \\
\hline STORY 27 & $\mathrm{~B} 1$ & DEAD & 30.667 & -24.482 & 711.415 \\
\hline STORY27 & $\mathrm{B} 1$ & DEAD & 51.333 & -22.060 & 1192.344 \\
\hline STORY27 & $\mathrm{B} 1$ & DEAD & 72.000 & -19.638 & 1623.224 \\
\hline STORY27 & $\mathrm{B} 1$ & DEAD & 72.000 & -13.208 & 1638.978 \\
\hline STORY27 & B1 & DEAD & 96.000 & -10.395 & 1922.212 \\
\hline STORY27 & $\mathrm{B} 1$ & DEAD & 120.000 & -7.583 & 2137.950 \\
\hline STORY27 & $\mathrm{B} 1$ & DEAD & 144.000 & -4.771 & 2286.192 \\
\hline STORY27 & $\mathrm{B} 1$ & DEAD & 144.000 & 4.771 & 2286.192 \\
\hline STORY27 & $\mathrm{B} 1$ & DEAD & 168.000 & 7.583 & 2137.950 \\
\hline STORY27 & $\mathrm{B} 1$ & DEAD & 192.000 & 10.395 & 1922.212 \\
\hline STORY27 & $\mathrm{B} 1$ & DEAD & 216.000 & 13.208 & 1638.978 \\
\hline STORY27 & $\mathrm{B} 1$ & DEAD & 216.000 & 19.638 & 1623.224 \\
\hline STORY27 & B1 & DEAD & 236.667 & 22.060 & 1192.344 \\
\hline STORY27 & B1 & DEAD & 257.333 & 24.482 & 711.415 \\
\hline STORY27 & B1 & DEAD & 278.000 & 26.903 & 180.437 \\
\hline STORY27 & B1 & LIVE & 10.000 & -5.429 & 96.556 \\
\hline STORY27 & $\mathrm{B} 1$ & LIVE & 30.667 & -5.429 & 208.752 \\
\hline STORY27 & $\mathrm{B} 1$ & LIVE & 51.333 & -5.429 & 320.949 \\
\hline STORY27 & B1 & LIVE & 72.000 & -5.429 & 433.145 \\
\hline STORY 27 & B1 & LIVE & 72.000 & -2.336 & 437.994 \\
\hline STORY 27 & B1 & LIVE & 96.000 & -2.336 & 494.062 \\
\hline STORY27 & $\mathrm{B} 1$ & LIVE & 120.000 & -2.336 & 550.130 \\
\hline STORY27 & B1 & LIVE & 144.000 & -2.336 & 606.198 \\
\hline
\end{tabular}




\begin{tabular}{|c|c|c|c|c|c|}
\hline STORY27 & $\mathrm{B} 1$ & LIVE & 144.000 & 2.336 & 606.198 \\
\hline STORY27 & B1 & LIVE & 168.000 & 2.336 & 550.130 \\
\hline STORY27 & $\mathrm{B} 1$ & LIVE & 192.000 & 2.336 & 494.062 \\
\hline STORY27 & $\mathrm{B} 1$ & LIVE & 216.000 & 2.336 & 437.994 \\
\hline STORY27 & $\overline{B 1}$ & LIVE & 216.000 & 5.429 & 433.145 \\
\hline STORY27 & $\mathrm{B} 1$ & LIVE & 236.667 & 5.429 & 320.949 \\
\hline STORY27 & $\mathrm{B} 1$ & LIVE & 257.333 & 5.429 & 208.752 \\
\hline STORY27 & B1 & LIVE & 278.000 & 5.429 & 96.556 \\
\hline STORY27 & $\mathrm{B} 1$ & WIND & 10.000 & 65.583 & 8835.384 \\
\hline STORY27 & $\mathrm{B} 1$ & WIND & 30.667 & 65.583 & 7480.007 \\
\hline STORY27 & $\mathrm{B} 1$ & WIND & 51.333 & 65.583 & 6124.631 \\
\hline STORY27 & $\mathrm{B} 1$ & WIND & 72.000 & 65.583 & 4769.255 \\
\hline STORY27 & B1 & WIND & 72.000 & 65.305 & 4724.298 \\
\hline STORY27 & B1 & WIND & 96.000 & 65.305 & 3156.983 \\
\hline STORY27 & B1 & WIND & 120.000 & 65.305 & 1589.668 \\
\hline STORY27 & B1 & WIND & 144.000 & 65.305 & 22.353 \\
\hline STORY27 & $\mathrm{B} 1$ & WIND & 144.000 & 65.305 & -22.353 \\
\hline STORY27 & $\mathrm{B} 1$ & WIND & 168.000 & 65.305 & -1589.668 \\
\hline STORY27 & $\mathrm{B} 1$ & WIND & 192.000 & 65.305 & -3156.983 \\
\hline STORY27 & B1 & WIND & 216.000 & 65.305 & -4724.298 \\
\hline STORY27 & $\mathrm{B} 1$ & WIND & 216.000 & 65.583 & -4769.255 \\
\hline STORY27 & $\mathrm{B} 1$ & WIND & 236.667 & 65.583 & -6124.631 \\
\hline STORY27 & $\mathrm{B} 1$ & WIND & 257.333 & 65.583 & -7480.007 \\
\hline STORY27 & $\mathrm{B} 1$ & WIND & 278.000 & 65.583 & -8835.384 \\
\hline STORY26 & B1 & DEAD & 12.000 & -26.672 & 94.125 \\
\hline STORY26 & $\mathrm{B} 1$ & DEAD & 32.000 & -24.328 & 604.128 \\
\hline STORY26 & $\mathrm{B} 1$ & DEAD & 52.000 & -21.985 & 1067.259 \\
\hline STORY26 & $\mathrm{B} 1$ & DEAD & 72.000 & -19.641 & 1483.518 \\
\hline STORY26 & $\mathrm{B} 1$ & DEAD & 72.000 & -13.208 & 1499.271 \\
\hline STORY26 & $\mathrm{B} 1$ & DEAD & 96.000 & -10.395 & 1782.509 \\
\hline STORY26 & $\mathrm{B} 1$ & DEAD & 120.000 & -7.583 & 1998.252 \\
\hline STORY26 & $\mathrm{B} 1$ & DEAD & 144.000 & -4.771 & 2146.499 \\
\hline STORY26 & $\mathrm{B} 1$ & DEAD & 144.000 & 4.771 & 2146.499 \\
\hline STORY26 & $\mathrm{B} 1$ & DEAD & 168.000 & 7.583 & 1998.252 \\
\hline STORY26 & $\mathrm{B} 1$ & DEAD & 192.000 & 10.395 & 1782.509 \\
\hline STORY26 & $\mathrm{B} 1$ & DEAD & 216.000 & 13.208 & 1499.271 \\
\hline STORY26 & $\mathrm{B} 1$ & DEAD & 216.000 & 19.641 & 1483.518 \\
\hline STORY26 & $\mathrm{B} 1$ & DEAD & 236.000 & 21.985 & 1067.259 \\
\hline STORY26 & $\mathrm{B} 1$ & DEAD & 256.000 & 24.328 & 604.128 \\
\hline STORY26 & $\mathrm{B} 1$ & DEAD & 276.000 & 26.672 & 94.125 \\
\hline STORY26 & B1 & LIVE & 12.000 & -5.430 & 69.834 \\
\hline STORY26 & $\mathrm{B} 1$ & LIVE & 32.000 & $\begin{array}{l}-5.430 \\
\end{array}$ & 178.427 \\
\hline STORY26 & $\mathrm{B} 1$ & LIVE & 52.000 & -5.430 & 287.020 \\
\hline STORY26 & $\mathrm{B} 1$ & LIVE & 72.000 & -5.430 & 395.613 \\
\hline STORY26 & $\mathrm{B} 1$ & LIVE & 72.000 & -2.336 & 400.461 \\
\hline STORY26 & B1 & LIVE & 96.000 & -2.336 & 456.531 \\
\hline STORY26 & B1 & LIVE & 120.000 & -2.336 & 512.600 \\
\hline
\end{tabular}




\begin{tabular}{|c|c|c|c|c|c|}
\hline STORY26 & B1 & LIVE & 144.000 & -2.336 & 568.670 \\
\hline STORY26 & $\mathrm{B} 1$ & LIVE & 144.000 & 2.336 & 568.670 \\
\hline STORY26 & $\mathrm{B} 1$ & LIVE & 168.000 & 2.336 & 512.600 \\
\hline STORY26 & $\mathrm{B} 1$ & LIVE & 192.000 & 2.336 & 456.531 \\
\hline STORY26 & $\mathrm{B} 1$ & LIVE & 216.000 & 2.336 & 400.461 \\
\hline STORY26 & $\mathrm{B} 1$ & LIVE & 216.000 & 5.430 & 395.613 \\
\hline STORY26 & B1 & LIVE & 236.000 & 5.430 & 287.020 \\
\hline STORY26 & $\mathrm{B} 1$ & LIVE & 256.000 & 5.430 & 178.427 \\
\hline STORY26 & $\mathrm{B} 1$ & LIVE & 276.000 & 5.430 & 69.834 \\
\hline STORY26 & B1 & WIND & 12.000 & 87.089 & 11558.880 \\
\hline STORY26 & B1 & WIND & 32.000 & 87.089 & 9817.101 \\
\hline STORY26 & $\mathrm{B} 1$ & WIND & 52.000 & 87.089 & 8075.324 \\
\hline STORY26 & $\mathrm{B} 1$ & WIND & 72.000 & 87.089 & 6333.546 \\
\hline STORY26 & $\mathrm{B} 1$ & WIND & 72.000 & 86.724 & 6273.839 \\
\hline STORY26 & B1 & WIND & 96.000 & 86.724 & 4192.454 \\
\hline STORY26 & $\mathrm{B} 1$ & WIND & 120.000 & 86.724 & 2111.070 \\
\hline STORY26 & $\mathrm{B} 1$ & WIND & 144.000 & 86.724 & 29.686 \\
\hline STORY26 & B1 & WIND & 144.000 & 86.724 & -29.686 \\
\hline STORY26 & $\mathrm{B} 1$ & WIND & 168.000 & 86.724 & -2111.070 \\
\hline STORY26 & B1 & WIND & 192.000 & 86.724 & -4192.454 \\
\hline STORY26 & $\mathrm{B} 1$ & WIND & 216.000 & 86.724 & -6273.839 \\
\hline STORY26 & $\mathrm{B} 1$ & WIND & 216.000 & 87.089 & -6333.546 \\
\hline STORY26 & $\mathrm{B} 1$ & WIND & 236.000 & 87.089 & -8075.324 \\
\hline STORY26 & $\mathrm{B} 1$ & WIND & 256.000 & 87.089 & -9817.101 \\
\hline STORY26 & $\mathrm{B} 1$ & WIND & 276.000 & 87.089 & -11558.880 \\
\hline STORY25 & $\mathrm{B} 1$ & DEAD & 12.000 & -26.675 & -28.544 \\
\hline STORY25 & B1 & DEAD & 32.000 & -24.331 & 481.512 \\
\hline STORY25 & $\mathrm{B} 1$ & DEAD & 52.000 & -21.987 & 944.695 \\
\hline STORY25 & B1 & DEAD & 72.000 & -19.644 & 1361.007 \\
\hline STORY25 & $\mathrm{B} 1$ & DEAD & 72.000 & -13.208 & 1376.757 \\
\hline STORY25 & $\mathrm{B} 1$ & DEAD & 96.000 & -10.396 & 1660.000 \\
\hline STORY25 & $\mathrm{B} 1$ & DEAD & 120.000 & -7.583 & 1875.747 \\
\hline STORY25 & $\mathrm{B} 1$ & DEAD & 144.000 & -4.771 & 2023.998 \\
\hline STORY25 & $\mathrm{B} 1$ & DEAD & 144.000 & 4.771 & 2023.998 \\
\hline STORY25 & $\mathrm{B} 1$ & DEAD & 168.000 & 7.583 & 1875.747 \\
\hline STORY25 & $\mathrm{B} 1$ & DEAD & 192.000 & 10.396 & 1660.000 \\
\hline STORY25 & $\mathrm{B} 1$ & DEAD & 216.000 & 13.208 & 1376.757 \\
\hline STORY25 & $\mathrm{B} 1$ & DEAD & 216.000 & 19.644 & 1361.007 \\
\hline STORY25 & $\mathrm{B} 1$ & DEAD & 236.000 & 21.987 & 944.695 \\
\hline STORY25 & $\mathrm{B} 1$ & DEAD & 256.000 & 24.331 & 481.512 \\
\hline STORY25 & $\mathrm{B} 1$ & DEAD & 276.000 & 26.675 & -28.544 \\
\hline STORY25 & $\mathrm{B} 1$ & LIVE & 12.000 & -5.430 & 36.879 \\
\hline STORY25 & $\mathrm{B} 1$ & LIVE & 32.000 & -5.430 & 145.486 \\
\hline STORY25 & $\mathrm{B} 1$ & LIVE & 52.000 & -5.430 & 254.093 \\
\hline STORY25 & $\mathrm{B} 1$ & LIVE & 72.000 & -5.430 & 362.700 \\
\hline STORY25 & $\mathrm{B} 1$ & LIVE & 72.000 & -2.336 & 367.548 \\
\hline STORY25 & $\mathrm{B} 1$ & LIVE & 96.000 & -2.336 & 423.619 \\
\hline
\end{tabular}




\begin{tabular}{|c|c|c|c|c|c|}
\hline STORY25 & B1 & LIVE & 120.000 & -2.336 & 479.689 \\
\hline STORY25 & B1 & LIVE & 144.000 & -2.336 & 535.760 \\
\hline STORY25 & B1 & LIVE & 144.000 & 2.336 & 535.760 \\
\hline STORY25 & B1 & LIVE & 168.000 & 2.336 & 479.689 \\
\hline STORY25 & B1 & LIVE & 192.000 & 2.336 & 423.619 \\
\hline STORY25 & B1 & LIVE & 216.000 & 2.336 & 367.548 \\
\hline STORY25 & B1 & LIVE & 216.000 & 5.430 & 362.700 \\
\hline STORY25 & B1 & LIVE & 236.000 & 5.430 & 254.093 \\
\hline STORY25 & B1 & LIVE & 256.000 & 5.430 & 145.486 \\
\hline STORY25 & $\mathrm{BI}$ & LIVE & 276.000 & 5.430 & 36.879 \\
\hline STORY25 & $\mathrm{B} 1$ & WIND & 12.000 & 108.264 & 14369.650 \\
\hline STORY25 & B1 & WIND & 32.000 & 108.264 & 12204.370 \\
\hline STORY25 & B1 & WIND & 52.000 & 108.264 & 10039.090 \\
\hline STORY25 & B1 & WIND & 72.000 & 108.264 & 7873.816 \\
\hline STORY25 & B1 & WIND & 72.000 & 107.815 & 7799.584 \\
\hline STORY25 & $\mathrm{B} 1$ & WIND & 96.000 & 107.815 & 5212.024 \\
\hline STORY25 & $\mathrm{B} 1$ & WIND & 120.000 & 107.815 & 2624.465 \\
\hline STORY25 & B1 & WIND & 144.000 & 107.815 & 36.905 \\
\hline STORY25 & $\mathrm{B} 1$ & WIND & 144.000 & 107.815 & -36.905 \\
\hline STORY25 & $\mathrm{B} 1$ & WIND & 168.000 & 107.815 & -2624.465 \\
\hline STORY25 & $\mathrm{B} 1$ & WIND & 192.000 & 107.815 & -5212.024 \\
\hline STORY25 & $\mathrm{B} 1$ & WIND & 216.000 & 107.815 & -7799.584 \\
\hline STORY25 & $\mathrm{B} 1$ & WIND & 216.000 & 108.264 & -7873.816 \\
\hline STORY25 & B1 & WIND & 236.000 & 108.264 & -10039.090 \\
\hline STORY25 & B1 & WIND & 256.000 & 108.264 & -12204.370 \\
\hline STORY25 & B1 & WIND & 276.000 & 108.264 & -14369.650 \\
\hline STORY24 & B1 & DEAD & 13.000 & -26.559 & -69.052 \\
\hline STORY24 & $\mathrm{B} 1$ & DEAD & 32.667 & -24.254 & 430.611 \\
\hline STORY24 & $\mathrm{B} 1$ & DEAD & 52.333 & -21.950 & 884.951 \\
\hline STORY24 & B1 & DEAD & 72.000 & -19.645 & 1293.968 \\
\hline STORY24 & B1 & DEAD & 72.000 & -13.208 & 1309.718 \\
\hline STORY24 & B1 & DEAD & 96.000 & -10.396 & 1592.963 \\
\hline STORY24 & $\mathrm{B} 1$ & DEAD & 120.000 & -7.583 & 1808.712 \\
\hline STORY24 & B1 & DEAD & 144.000 & -4.771 & 1956.966 \\
\hline STORY24 & $\mathrm{B} 1$ & DEAD & 144.000 & 4.771 & 1956.966 \\
\hline STORY24 & $\mathrm{B} 1$ & DEAD & 168.000 & 7.583 & 1808.712 \\
\hline STORY24 & $\mathrm{B} 1$ & DEAD & 192.000 & 10.396 & 1592.963 \\
\hline STORY24 & B1 & DEAD & 216.000 & 13.208 & 1309.718 \\
\hline STORY24 & B1 & DEAD & 216.000 & 19.645 & 1293.968 \\
\hline STORY24 & $\mathrm{B} 1$ & DEAD & 235.667 & 21.950 & 884.951 \\
\hline STORY24 & $\mathrm{B} 1$ & DEAD & 255.333 & 24.254 & 430.611 \\
\hline STORY24 & $\mathrm{B} 1$ & DEAD & 275.000 & 26.559 & -69.052 \\
\hline STORY24 & $\mathrm{B} 1$ & LIVE & 13.000 & -5.431 & 24.276 \\
\hline STORY24 & $\mathrm{B} 1$ & LIVE & 32.667 & -5.431 & 131.081 \\
\hline STORY24 & $\mathrm{B} 1$ & LIVE & 52.333 & -5.431 & 237.885 \\
\hline STORY24 & $\mathrm{B} 1$ & LIVE & 72.000 & -5.431 & 344.690 \\
\hline STORY24 & $\mathrm{B} 1$ & LIVE & 72.000 & -2.336 & 349.538 \\
\hline
\end{tabular}




\begin{tabular}{|c|c|c|c|c|c|}
\hline STORY24 & B1 & LIVE & 96.000 & -2.336 & 405.609 \\
\hline STORY24 & $\mathrm{B} 1$ & LIVE & 120.000 & -2.336 & 461.680 \\
\hline STORY24 & $\mathrm{B} 1$ & LIVE & 144.000 & -2.336 & 517.751 \\
\hline STORY24 & B1 & LIVE & 144.000 & 2.336 & 517.751 \\
\hline STORY24 & $\mathrm{B} 1$ & LIVE & 168.000 & 2.336 & 461.680 \\
\hline STORY24 & $\mathrm{B} 1$ & LIVE & 192.000 & 2.336 & 405.609 \\
\hline STORY24 & B1 & LIVE & 216.000 & 2.336 & 349.538 \\
\hline STORY24 & $\mathrm{B} 1$ & LIVE & 216.000 & 5.431 & 344.690 \\
\hline STORY24 & B1 & LIVE & 235.667 & 5.431 & 237.885 \\
\hline STORY24 & B1 & LIVE & 255.333 & 5.431 & 131.081 \\
\hline STORY24 & $\mathrm{B} 1$ & LIVE & 275.000 & 5.431 & 24.276 \\
\hline STORY24 & $\mathrm{B} 1$ & WIND & 13.000 & 129.271 & 17028.850 \\
\hline STORY24 & $\mathrm{B} 1$ & WIND & 32.667 & 129.271 & 14486.520 \\
\hline STORY24 & $\mathrm{B} 1$ & WIND & 52.333 & 129.271 & 11944.200 \\
\hline STORY24 & $\mathrm{B} 1$ & WIND & 72.000 & 129.271 & 9401.872 \\
\hline STORY24 & $\mathrm{B} 1$ & WIND & 72.000 & 128.738 & 9313.230 \\
\hline STORY24 & $\mathrm{B} 1$ & WIND & 96.000 & 128.738 & 6223.510 \\
\hline STORY24 & B1 & WIND & 120.000 & 128.738 & 3133.789 \\
\hline STORY24 & $\mathrm{B} 1$ & WIND & 144.000 & 128.738 & 44.068 \\
\hline STORY24 & $\mathrm{B} 1$ & WIND & 144.000 & 128.738 & -44.068 \\
\hline STORY24 & $\mathrm{B} 1$ & WIND & 168.000 & 128.738 & -3133.789 \\
\hline STORY24 & $\mathrm{B} 1$ & WIND & 192.000 & 128.738 & -6223.510 \\
\hline STORY24 & $\mathrm{B} 1$ & WIND & 216.000 & 128.738 & -9313.230 \\
\hline STORY24 & $\mathrm{B} 1$ & WIND & 216.000 & 129.271 & -9401.872 \\
\hline STORY24 & B1 & WIND & 235.667 & 129.271 & -11944.200 \\
\hline STORY24 & $\mathrm{B} 1$ & WIND & 255.333 & 129.271 & -14486.520 \\
\hline STORY24 & B1 & WIND & 275.000 & 129.271 & -17028.850 \\
\hline STORY23 & B1 & DEAD & 13.000 & -26.560 & -133.690 \\
\hline STORY23 & B1 & DEAD & 32.667 & -24.256 & 366.000 \\
\hline STORY23 & B1 & DEAD & 52.333 & -21.951 & 820.367 \\
\hline STORY23 & B1 & DEAD & 72.000 & -19.647 & 1229.411 \\
\hline STORY23 & B1 & DEAD & 72.000 & -13.208 & 1245.160 \\
\hline STORY23 & $\mathrm{B} 1$ & DEAD & 96.000 & -10.396 & 1528.407 \\
\hline STORY23 & B1 & DEAD & 120.000 & -7.583 & 1744.159 \\
\hline STORY23 & B1 & DEAD & 144.000 & -4.771 & 1892.414 \\
\hline STORY23 & $\mathrm{B} 1$ & DEAD & 144.000 & 4.771 & 1892.414 \\
\hline STORY23 & $\mathrm{B} 1$ & DEAD & 168.000 & 7.583 & 1744.159 \\
\hline STORY23 & $\mathrm{B} 1$ & DEAD & 192.000 & 10.396 & 1528.407 \\
\hline STORY23 & B1 & DEAD & 216.000 & 13.208 & 1245.160 \\
\hline STORY23 & $\mathrm{B} 1$ & DEAD & 216.000 & 19.647 & 1229.411 \\
\hline STORY23 & B1 & DEAD & 235.667 & 21.951 & 820.367 \\
\hline STORY 23 & $\mathrm{~B} 1$ & DEAD & 255.333 & 24.256 & 366.000 \\
\hline STORY23 & $\mathrm{B} 1$ & DEAD & 275.000 & 26.560 & -133.690 \\
\hline STORY23 & B1 & LIVE & 13.000 & -5.431 & 6.911 \\
\hline STORY 23 & B1 & LIVE & 32.667 & -5.431 & 113.723 \\
\hline STORY23 & $\mathrm{B} 1$ & LIVE & 52.333 & -5.431 & 220.535 \\
\hline STORY23 & B1 & LIVE & 72.000 & -5.431 & 327.347 \\
\hline
\end{tabular}




\begin{tabular}{|c|c|c|c|c|c|}
\hline STORY23 & B1 & LIVE & 72.000 & -2.336 & 332.194 \\
\hline STORY23 & $\mathrm{B} 1$ & LIVE & 96.000 & -2.336 & 388.266 \\
\hline STORY23 & $\mathrm{B} 1$ & LIVE & 120.000 & -2.336 & 444.338 \\
\hline STORY23 & $\mathrm{B} 1$ & LIVE & 144.000 & -2.336 & 500.410 \\
\hline STORY23 & $\mathrm{B} 1$ & LIVE & 144.000 & 2.336 & 500.410 \\
\hline STORY23 & $\mathrm{B} 1$ & LIVE & 168.000 & 2.336 & 444.338 \\
\hline STORY23 & $\mathrm{B} 1$ & LIVE & 192.000 & 2.336 & 388.266 \\
\hline STORY23 & $\mathrm{B} 1$ & LIVE & 216.000 & 2.336 & 332.194 \\
\hline STORY23 & $\mathrm{B} 1$ & LIVE & 216.000 & 5.431 & 327.347 \\
\hline STORY23 & $\mathrm{B} 1$ & LIVE & 235.667 & 5.431 & 220.535 \\
\hline STORY23 & $\mathrm{B} 1$ & LIVE & 255.333 & 5.431 & 113.723 \\
\hline STORY23 & $\mathrm{B} 1$ & LIVE & $27 \overline{5.000}$ & 5.431 & 6.911 \\
\hline STORY23 & $\mathrm{B} 1$ & WIND & 13.000 & 150.103 & 19773.350 \\
\hline STORY23 & B1 & WIND & 32.667 & 150.103 & 16821.320 \\
\hline STORY23 & $\mathrm{B} 1$ & WIND & 52.333 & 150.103 & 13869.300 \\
\hline STORY23 & B1 & WIND & 72.000 & 150.103 & 10917.270 \\
\hline STORY23 & B1 & WIND & 72.000 & 149.488 & 10814.340 \\
\hline STORY23 & B1 & WIND & 96.000 & 149.488 & 7226.614 \\
\hline STORY23 & $\mathrm{B} 1$ & WIND & 120.000 & 149.488 & 3638.893 \\
\hline STORY23 & $\mathrm{B} 1$ & WIND & 144.000 & 149.488 & 51.171 \\
\hline STORY23 & $\mathrm{B} 1$ & WIND & 144.000 & 149.488 & -51.171 \\
\hline STORY23 & $\mathrm{B} 1$ & WIND & 168.000 & 149.488 & -3638.893 \\
\hline STORY23 & $\mathrm{B} 1$ & WIND & 192.000 & 149.488 & -7226.614 \\
\hline STORY23 & $\mathrm{B} 1$ & WIND & 216.000 & 149.488 & -10814.340 \\
\hline STORY23 & $\mathrm{B} 1$ & WIND & 216.000 & 150.103 & -10917.270 \\
\hline STORY23 & $\mathrm{B} 1$ & WIND & 235.667 & 150.103 & -13869.300 \\
\hline STORY23 & $\mathrm{B} 1$ & WIND & 255.333 & 150.103 & -16821.320 \\
\hline STORY23 & $\mathrm{B} 1$ & WIND & 275.000 & 150.103 & -19773.350 \\
\hline STORY22 & $\mathrm{B} 1$ & DEAD & 14.000 & -26.444 & -168.167 \\
\hline STORY22 & $\mathrm{B} 1$ & DEAD & 33.333 & -24.179 & 321.190 \\
\hline STORY22 & $\mathrm{B} 1$ & DEAD & 52.667 & -21.913 & 766.749 \\
\hline STORY22 & $\mathrm{B} 1$ & DEAD & 72.000 & -19.648 & 1168.508 \\
\hline STORY22 & $\mathrm{B} 1$ & DEAD & 72.000 & -13.208 & 1184.255 \\
\hline STORY22 & $\mathrm{B} 1$ & DEAD & 96.000 & -10.396 & 1467.505 \\
\hline STORY22 & $\mathrm{B} 1$ & DEAD & 120.000 & -7.584 & 1683.259 \\
\hline STORY22 & $\mathrm{B} 1$ & DEAD & 144.000 & -4.771 & 1831.517 \\
\hline STORY22 & $\mathrm{B} 1$ & DEAD & 144.000 & 4.771 & 1831.517 \\
\hline STORY22 & $\mathrm{B} 1$ & DEAD & 168.000 & 7.584 & 1683.259 \\
\hline STORY22 & B1 & DEAD & 192.000 & 10.396 & 1467.505 \\
\hline STORY22 & $\mathrm{B} 1$ & DEAD & 216.000 & 13.208 & 1184.255 \\
\hline STORY22 & B1 & DEAD & 216.000 & 19.648 & 1168.508 \\
\hline STORY22 & $\mathrm{B} 1$ & DEAD & 235.333 & 21.913 & 766.749 \\
\hline STORY22 & B1 & DEAD & 254.667 & 24.179 & 321.190 \\
\hline STORY 22 & $\mathrm{~B} 1$ & DEAD & 274.000 & 26.444 & -168.167 \\
\hline STORY 22 & $\mathrm{~B} 1$ & LIVE & 14.000 & -5.431 & -4.040 \\
\hline STORY 22 & $\mathrm{~B} 1$ & LIVE & 33.333 & -5.431 & 100.968 \\
\hline STORY22 & $\mathrm{B} 1$ & LIVE & 52.667 & -5.431 & 205.977 \\
\hline
\end{tabular}




\begin{tabular}{|c|c|c|c|c|c|}
\hline STORY22 & $\mathrm{B} 1$ & LIVE & 72.000 & -5.431 & 310.985 \\
\hline STORY22 & B1 & LIVE & 72.000 & -2.336 & 315.832 \\
\hline STORY22 & B1 & LIVE & 96.000 & -2.336 & 371.905 \\
\hline STORY22 & B1 & LIVE & 120.000 & -2.336 & 427.977 \\
\hline STORY22 & B1 & LIVE & 144.000 & -2.336 & 484.049 \\
\hline STORY22 & B1 & LIVE & 144.000 & 2.336 & 484.049 \\
\hline STORY22 & B1 & LIVE & 168.000 & 2.336 & 427.977 \\
\hline STORY22 & B1 & LIVE & 192.000 & 2.336 & 371.905 \\
\hline STORY22 & $\mathrm{B} 1$ & LIVE & 216.000 & 2.336 & 315.832 \\
\hline STORY22 & B1 & LIVE & 216.000 & 5.431 & 310.985 \\
\hline STORY22 & B1 & LIVE & 235.333 & 5.431 & 205.977 \\
\hline STORY22 & $\mathrm{B} 1$ & LIVE & 254.667 & 5.431 & 100.968 \\
\hline STORY22 & B1 & LIVE & 274.000 & 5.431 & -4.040 \\
\hline STORY22 & B1 & WIND & 14.000 & 170.547 & 22296.220 \\
\hline STORY22 & B1 & WIND & 33.333 & 170.547 & 18998.980 \\
\hline STORY22 & B1 & WIND & 52.667 & 170.547 & 15701.750 \\
\hline STORY22 & $\mathrm{B} 1$ & WIND & 72.000 & 170.547 & 12404.510 \\
\hline STORY22 & $\mathrm{Bl}$ & WIND & 72.000 & 169.853 & 12287.550 \\
\hline STORY22 & B1 & WIND & 96.000 & 169.853 & 8211.083 \\
\hline STORY22 & B1 & WIND & 120.000 & 169.853 & 4134.613 \\
\hline STORY22 & B1 & WIND & 144.000 & 169.853 & 58.143 \\
\hline STORY22 & B1 & WIND & 144.000 & 169.853 & -58.143 \\
\hline STORY22 & B1 & WIND & 168.000 & 169.853 & -4134.613 \\
\hline STORY22 & B1 & WIND & 192.000 & 169.853 & -8211.083 \\
\hline STORY22 & $\mathrm{B} 1$ & WIND & 216.000 & 169.853 & -12287.550 \\
\hline STORY22 & $\mathrm{B} 1$ & WIND & 216.000 & 170.547 & -12404.510 \\
\hline STORY22 & B1 & WIND & 235.333 & 170.547 & -15701.750 \\
\hline STORY22 & B1 & WIND & 254.667 & 170.547 & -18998.980 \\
\hline STORY22 & B1 & WIND & 274.000 & 170.547 & -22296.220 \\
\hline STORY21 & B1 & DEAD & 15.000 & -26.330 & -281.020 \\
\hline STORY21 & B1 & DEAD & 34.000 & -24.104 & 198.101 \\
\hline STORY21 & B1 & DEAD & 53.000 & -21.877 & 634.921 \\
\hline STORY21 & B1 & DEAD & 72.000 & -19.651 & 1029.439 \\
\hline STORY21 & B1 & DEAD & 72.000 & -13.208 & 1045.184 \\
\hline STORY21 & $\mathrm{B} 1$ & DEAD & 96.000 & -10.396 & 1328.439 \\
\hline STORY21 & $\mathrm{B} 1$ & DEAD & 120.000 & -7.584 & 1544.197 \\
\hline STORY21 & B1 & DEAD & 144.000 & -4.771 & 1692.460 \\
\hline STORY21 & B1 & DEAD & 144.000 & 4.771 & 1692.460 \\
\hline STORY21 & B1 & DEAD & 168.000 & 7.584 & 1544.197 \\
\hline STORY 21 & $\mathrm{~B} 1$ & DEAD & 192.000 & 10.396 & 1328.439 \\
\hline STORY21 & $\mathrm{Bl}$ & DEAD & 216.000 & 13.208 & 1045.184 \\
\hline STORY 21 & B1 & DEAD & 216.000 & 19.651 & 1029.439 \\
\hline STORY21 & $\mathrm{B} 1$ & DEAD & 235.000 & 21.877 & 634.921 \\
\hline STORY21 & B1 & DEAD & 254.000 & 24.104 & 198.101 \\
\hline STORY 21 & $\mathrm{~B} 1$ & DEAD & 273.000 & 26.330 & -281.020 \\
\hline STORY21 & $\mathrm{B} 1$ & LIVE & 15.000 & -5.432 & -36.015 \\
\hline STORY21 & B1 & LIVE & 34.000 & -5.432 & 67.198 \\
\hline
\end{tabular}




\begin{tabular}{|c|c|c|c|c|c|}
\hline STORY21 & B1 & LIVE & 53.000 & -5.432 & 170.411 \\
\hline STORY21 & $\mathrm{B} 1$ & LIVE & 72.000 & -5.432 & 273.624 \\
\hline STORY21 & $\mathrm{B} 1$ & LIVE & 72.000 & -2.336 & 278.471 \\
\hline STORY21 & $\mathrm{B} 1$ & LIVE & 96.000 & -2.336 & 334.544 \\
\hline STORY21 & $\mathrm{B} 1$ & LIVE & 120.000 & -2.336 & 390.618 \\
\hline STORY21 & $\mathrm{B} 1$ & LIVE & 144.000 & -2.336 & 446.692 \\
\hline STORY21 & $\mathrm{B} 1$ & LIVE & 144.000 & 2.336 & 446.692 \\
\hline STORY21 & $\mathrm{B} 1$ & LIVE & 168.000 & 2.336 & 390.618 \\
\hline STORY21 & $\mathrm{B} 1$ & LIVE & 192.000 & 2.336 & 334.544 \\
\hline STORY21 & B1 & LIVE & 216.000 & 2.336 & 278.471 \\
\hline STORY21 & $\mathrm{B} 1$ & LIVE & 216.000 & 5.432 & 273.624 \\
\hline STORY21 & $\mathrm{B} 1$ & LIVE & 235.000 & 5.432 & 170.411 \\
\hline STORY21 & $\mathrm{B} 1$ & LIVE & 254.000 & 5.432 & 67.198 \\
\hline STORY21 & $\mathrm{B} 1$ & LIVE & 273.000 & 5.432 & -36.015 \\
\hline STORY21 & $\mathrm{B} 1$ & WIND & 15.000 & 190.764 & 24749.370 \\
\hline STORY21 & $\mathrm{B} 1$ & WIND & 34.000 & 190.764 & 21124.850 \\
\hline STORY21 & $\mathrm{B} 1$ & WIND & 53.000 & 190.764 & 17500.330 \\
\hline STORY21 & $\mathrm{B} 1$ & WIND & 72.000 & 190.764 & 13875.820 \\
\hline STORY21 & B1 & WIND & 72.000 & 189.999 & 13744.970 \\
\hline STORY21 & $\mathrm{B} 1$ & WIND & 96.000 & 189.999 & 9184.996 \\
\hline STORY21 & $\mathrm{B} 1$ & WIND & 120.000 & 189.999 & 4625.018 \\
\hline STORY21 & $\mathrm{B} 1$ & WIND & 144.000 & 189.999 & 65.040 \\
\hline STORY21 & $\mathrm{B} 1$ & WIND & 144.000 & 189.999 & -65.040 \\
\hline STORY21 & B1 & WIND & 168.000 & 189.999 & -4625.018 \\
\hline STORY21 & $\mathrm{B} 1$ & WIND & 192.000 & 189.999 & -9184.996 \\
\hline STORY21 & B1 & WIND & 216.000 & 189.999 & -13744.970 \\
\hline STORY21 & $\mathrm{B} 1$ & WIND & 216.000 & 190.764 & -13875.820 \\
\hline STORY21 & $\mathrm{B} 1$ & WIND & 235.000 & 190.764 & -17500.330 \\
\hline STORY21 & $\mathrm{B} 1$ & WIND & 254.000 & 190.764 & -21124.850 \\
\hline STORY21 & $\mathrm{B} 1$ & WIND & 273.000 & 190.764 & -24749.370 \\
\hline STORY20 & $\mathrm{B} 1$ & DEAD & 15.000 & -26.331 & -332.470 \\
\hline STORY20 & B1 & DEAD & 34.000 & -24.105 & 146.673 \\
\hline STORY20 & $\mathrm{B} 1$ & DEAD & 53.000 & -21.878 & 583.514 \\
\hline STORY20 & $\mathrm{B} 1$ & DEAD & 72.000 & -19.652 & 978.052 \\
\hline STORY20 & $\mathrm{B} 1$ & DEAD & 72.000 & -13.209 & 993.797 \\
\hline STORY20 & $\mathrm{B} 1$ & DEAD & 96.000 & -10.396 & 1277.053 \\
\hline STORY20 & $\mathrm{B} 1$ & DEAD & 120.000 & $\begin{array}{l}-7.584 \\
\end{array}$ & 1492.813 \\
\hline STORY20 & $\mathrm{B} 1$ & DEAD & 144.000 & -4.772 & 1641.078 \\
\hline STORY20 & $\mathrm{B} 1$ & DEAD & 144.000 & 4.772 & 1641.078 \\
\hline STORY20 & $\mathrm{B} 1$ & DEAD & 168.000 & 7.584 & 1492.813 \\
\hline STORY20 & $\mathrm{B} 1$ & DEAD & 192.000 & 10.396 & 1277.053 \\
\hline STORY20 & $\mathrm{B} 1$ & DEAD & 216.000 & 13.209 & 993.797 \\
\hline STORY20 & $\mathrm{B} 1$ & DEAD & 216.000 & 19.652 & 978.052 \\
\hline STORY20 & $\overline{\mathrm{B} 1}$ & DEAD & 235.000 & 21.878 & 583.514 \\
\hline STORY20 & $\mathrm{B} 1$ & DEAD & 254.000 & 24.105 & 146.673 \\
\hline STORY20 & $\mathrm{B} 1$ & DEAD & 273.000 & 26.331 & -332.470 \\
\hline STORY20 & $\mathrm{B} 1$ & LIVE & 15.000 & -5.433 & -49.837 \\
\hline
\end{tabular}




\begin{tabular}{|c|c|c|c|c|c|}
\hline STORY20 & $\mathrm{B} 1$ & LIVE & 34.000 & -5.433 & 53.382 \\
\hline STORY20 & $\mathrm{B} 1$ & LIVE & 53.000 & -5.433 & 156.600 \\
\hline STORY20 & $\mathrm{B} 1$ & LIVE & 72.000 & -5.433 & 259.819 \\
\hline STORY20 & $\mathrm{B} 1$ & LIVE & 72.000 & -2.336 & 264.665 \\
\hline STORY20 & $\mathrm{B} 1$ & LIVE & 96.000 & -2.336 & 320.739 \\
\hline STORY20 & $\mathrm{B} 1$ & LIVE & 120.000 & -2.336 & 376.814 \\
\hline STORY20 & $\mathrm{B} 1$ & LIVE & 144.000 & -2.336 & 432.888 \\
\hline STORY20 & $\mathrm{B} 1$ & LIVE & 144.000 & 2.336 & 432.888 \\
\hline STORY20 & $\mathrm{B} 1$ & LIVE & 168.000 & 2.336 & 376.814 \\
\hline STORY20 & B1 & LIVE & 192.000 & 2.336 & 320.739 \\
\hline STORY20 & $\mathrm{B} 1$ & LIVE & 216.000 & 2.336 & 264.665 \\
\hline STORY20 & $\mathrm{B} 1$ & LIVE & 216.000 & 5.433 & 259.819 \\
\hline STORY20 & $\mathrm{B} 1$ & LIVE & 235.000 & 5.433 & 156.600 \\
\hline STORY20 & $\mathrm{B} 1$ & LIVE & 254.000 & 5.433 & 53.382 \\
\hline STORY20 & $\mathrm{B} 1$ & LIVE & 273.000 & 5.433 & -49.837 \\
\hline STORY20 & $\mathrm{B} 1$ & WIND & 15.000 & 211.022 & 27377.980 \\
\hline STORY20 & $\mathrm{B} 1$ & WIND & 34.000 & 211.022 & 23368.560 \\
\hline STORY20 & $\mathrm{B} 1$ & WIND & 53.000 & 211.022 & 19359.140 \\
\hline STORY20 & $\mathrm{B} 1$ & WIND & 72.000 & 211.022 & 15349.720 \\
\hline STORY20 & $\mathrm{B} 1$ & WIND & 72.000 & 210.181 & 15204.970 \\
\hline STORY20 & $\mathrm{B} 1$ & WIND & 96.000 & 210.181 & 10160.630 \\
\hline STORY20 & $\mathrm{B} 1$ & WIND & 120.000 & 210.181 & 5116.292 \\
\hline STORY20 & $\mathrm{B} 1$ & WIND & 144.000 & 210.181 & 71.950 \\
\hline STORY20 & $\mathrm{B} 1$ & WIND & 144.000 & 210.181 & -71.950 \\
\hline STORY20 & $\mathrm{B} 1$ & WIND & 168.000 & 210.181 & -5116.292 \\
\hline STORY20 & $\mathrm{B} 1$ & WIND & 192.000 & 210.181 & -10160.630 \\
\hline STORY20 & $\mathrm{B} 1$ & WIND & 216.000 & 210.181 & -15204.970 \\
\hline STORY20 & $\mathrm{B} 1$ & WIND & 216.000 & 211.022 & -15349.720 \\
\hline STORY20 & $\mathrm{B} 1$ & WIND & 235.000 & 211.022 & -19359.140 \\
\hline STORY20 & $\mathrm{B} 1$ & WIND & 254.000 & 211.022 & -23368.560 \\
\hline STORY20 & $\mathrm{B} 1$ & WIND & 273.000 & 211.022 & -27377.980 \\
\hline STORY19 & $\mathrm{B} 1$ & DEAD & 16.000 & -26.215 & -348.061 \\
\hline STORY 19 & $\mathrm{~B} 1$ & DEAD & 34.667 & -24.028 & 120.870 \\
\hline STORY 19 & $\mathrm{~B} 1$ & DEAD & 53.333 & -21.840 & 548.970 \\
\hline STORY19 & $\mathrm{B} 1$ & DEAD & 72.000 & -19.653 & 936.239 \\
\hline STORY19 & $\mathrm{B} 1$ & DEAD & 72.000 & -13.209 & 951.983 \\
\hline STORY19 & $\mathrm{B} 1$ & DEAD & 96.000 & -10.396 & 1235.240 \\
\hline STORY19 & $\mathrm{B} 1$ & DEAD & 120.000 & -7.584 & 1451.002 \\
\hline STORY 19 & $\mathrm{~B} 1$ & DEAD & 144.000 & -4.772 & 1599.268 \\
\hline STORY19 & $\mathrm{B} 1$ & DEAD & 144.000 & 4.772 & 1599.268 \\
\hline STORY 19 & $\mathrm{~B} 1$ & DEAD & 168.000 & 7.584 & 1451.002 \\
\hline STORY19 & $\mathrm{B} 1$ & DEAD & 192.000 & 10.396 & 1235.240 \\
\hline STORY19 & $\mathrm{B} 1$ & DEAD & 216.000 & 13.209 & 951.983 \\
\hline STORY19 & $\mathrm{B} 1$ & DEAD & 216.000 & 19.653 & 936.239 \\
\hline STORY19 & $\mathrm{B} 1$ & DEAD & 234.667 & 21.840 & 548.970 \\
\hline STORY19 & $\mathrm{B} 1$ & DEAD & 253.333 & 24.028 & 120.870 \\
\hline STORY19 & $\mathrm{B} 1$ & DEAD & 272.000 & 26.215 & -348.061 \\
\hline
\end{tabular}




\begin{tabular}{|c|c|c|c|c|c|}
\hline STORY19 & B1 & LIVE & 16.000 & -5.433 & -55.651 \\
\hline STORY 19 & B1 & LIVE & 34.667 & -5.433 & 45.761 \\
\hline STORY 19 & B1 & LIVE & 53.333 & -5.433 & 147.173 \\
\hline STORY 19 & $\mathrm{~B} 1$ & LIVE & 72.000 & -5.433 & 248.586 \\
\hline STORY 19 & $\mathrm{~B} 1$ & LIVE & 72.000 & -2.336 & 253.432 \\
\hline STORY 19 & B1 & LIVE & 96.000 & -2.336 & 309.506 \\
\hline STORY19 & $\mathrm{B} 1$ & LIVE & 120.000 & -2.336 & 365.581 \\
\hline STORY 19 & B1 & LIVE & 144.000 & -2.336 & 421.656 \\
\hline STORY 19 & $\mathrm{~B} 1$ & LIVE & 144.000 & 2.336 & 421.656 \\
\hline STORY 19 & $\mathrm{~B} 1$ & LIVE & 168.000 & 2.336 & 365.581 \\
\hline STORY 19 & $\mathrm{~B} 1$ & LIVE & 192.000 & 2.336 & 309.506 \\
\hline STORY 19 & $\mathrm{~B} 1$ & LIVE & 216.000 & 2.336 & 253.432 \\
\hline STORY 19 & $\mathrm{~B} 1$ & LIVE & 216.000 & 5.433 & 248.586 \\
\hline STORY19 & $\mathrm{B} 1$ & LIVE & 234.667 & 5.433 & 147.173 \\
\hline STORY 19 & $\mathrm{~B} 1$ & LIVE & 253.333 & 5.433 & 45.761 \\
\hline STORY19 & $\mathrm{B} 1$ & LIVE & 272.000 & 5.433 & -55.651 \\
\hline STORY19 & $\mathrm{B} 1$ & WIND & 16.000 & 230.444 & 29667.790 \\
\hline STORY19 & $\mathrm{B} 1$ & WIND & 34.667 & 230.444 & 25366.160 \\
\hline STORY19 & $\mathrm{B} 1$ & WIND & 53.333 & 230.444 & 21064.540 \\
\hline STORY19 & $\mathrm{B} 1$ & WIND & 72.000 & 230.444 & 16762.900 \\
\hline STORY19 & $\mathrm{B} 1$ & WIND & 72.000 & 229.531 & 16604.830 \\
\hline STORY19 & $\mathrm{B} 1$ & WIND & 96.000 & 229.531 & 11096.080 \\
\hline STORY19 & $\mathrm{B} 1$ & WIND & 120.000 & 229.531 & 5587.327 \\
\hline STORY19 & B1 & WIND & 144.000 & 229.531 & 78.574 \\
\hline STORY19 & $\mathrm{Bl}$ & WIND & 144.000 & 229.531 & -78.574 \\
\hline STORY19 & $\mathrm{B} 1$ & WIND & 168.000 & 229.531 & -5587.327 \\
\hline STORY19 & $\mathrm{B} 1$ & WIND & 192.000 & 229.531 & -11096.080 \\
\hline STORY19 & $\mathrm{B} 1$ & WIND & 216.000 & 229.531 & -16604.830 \\
\hline STORY19 & $\mathrm{B} 1$ & WIND & 216.000 & 230.444 & -16762.900 \\
\hline STORY19 & B1 & WIND & 234.667 & 230.444 & -21064.540 \\
\hline STORY19 & $\mathrm{B} 1$ & WIND & 253.333 & 230.444 & -25366.160 \\
\hline STORY19 & $\mathrm{B} 1$ & WIND & 272.000 & 230.444 & -29667.790 \\
\hline STORY18 & $\mathrm{B} 1$ & DEAD & 18.000 & -25.984 & -471.052 \\
\hline STORY18 & $\mathrm{B} 1$ & DEAD & 36.000 & -23.875 & -22.317 \\
\hline STORY18 & $\mathrm{B} 1$ & DEAD & 54.000 & -21.766 & 388.451 \\
\hline STORY18 & $\mathrm{B} 1$ & DEAD & 72.000 & -19.657 & 761.254 \\
\hline STORY18 & $\mathrm{B} 1$ & DEAD & 72.000 & -13.209 & 776.995 \\
\hline STORY18 & $\mathrm{B} 1$ & DEAD & 96.000 & -10.396 & 1060.259 \\
\hline STORY18 & $\mathrm{B} 1$ & DEAD & 120.000 & -7.584 & 1276.027 \\
\hline STORY 18 & $\mathrm{~B} 1$ & DEAD & 144.000 & -4.772 & 1424.299 \\
\hline STORY 18 & $\mathrm{~B} 1$ & DEAD & 144.000 & 4.772 & 1424.299 \\
\hline STORY 18 & $\mathrm{~B} 1$ & DEAD & 168.000 & 7.584 & 1276.027 \\
\hline STORY 18 & $\mathrm{~B} 1$ & DEAD & 192.000 & 10.396 & 1060.259 \\
\hline STORY 18 & B1 & DEAD & 216.000 & 13.209 & 776.995 \\
\hline STORY 18 & B1 & DEAD & 216.000 & 19.657 & 761.254 \\
\hline STORY18 & $\mathrm{B} 1$ & DEAD & 234.000 & 21.766 & 388.451 \\
\hline STORY 18 & $\mathrm{~B} 1$ & DEAD & 252.000 & 23.875 & -22.317 \\
\hline
\end{tabular}




\begin{tabular}{|c|c|c|c|c|c|}
\hline STORY 18 & B1 & DEAD & 270.000 & 25.984 & -471.052 \\
\hline STORY18 & $\mathrm{B} 1$ & LIVE & 18.000 & -5.434 & -91.850 \\
\hline STORY18 & B1 & LIVE & 36.000 & -5.434 & 5.959 \\
\hline STORY18 & $\mathrm{B} 1$ & LIVE & 54.000 & -5.434 & 103.767 \\
\hline STORY 18 & $\mathrm{~B} 1$ & LIVE & 72.000 & -5.434 & 201.576 \\
\hline STORY18 & B1 & LIVE & 72.000 & -2.337 & 206.421 \\
\hline STORY18 & $\mathrm{B} 1$ & LIVE & 96.000 & -2.337 & 262.497 \\
\hline STORY18 & $\mathrm{B} 1$ & LIVE & 120.000 & -2.337 & 318.574 \\
\hline STORY18 & $\mathrm{B} 1$ & LIVE & 144.000 & -2.337 & 374.650 \\
\hline STORY 18 & $\mathrm{~B} 1$ & LIVE & 144.000 & 2.337 & 374.650 \\
\hline STORY18 & B1 & LIVE & 168.000 & 2.337 & 318.574 \\
\hline STORY18 & $\mathrm{B} 1$ & LIVE & 192.000 & 2.337 & 262.497 \\
\hline STORY18 & $\mathrm{B} 1$ & LIVE & 216.000 & 2.337 & 206.421 \\
\hline STORY18 & $\mathrm{B} 1$ & LIVE & 216.000 & 5.434 & 201.576 \\
\hline STORY18 & $\mathrm{B} 1$ & LIVE & 234.000 & 5.434 & 103.767 \\
\hline STORY18 & $\mathrm{B} 1$ & LIVE & 252.000 & 5.434 & 5.959 \\
\hline STORY 18 & $\mathrm{~B} 1$ & LIVE & 270.000 & 5.434 & -91.850 \\
\hline STORY18 & $\mathrm{B} 1$ & WIND & 18.000 & 249.263 & 31593.590 \\
\hline STORY18 & B1 & WIND & 36.000 & 249.263 & 27106.860 \\
\hline STORY18 & B1 & WIND & 54.000 & 249.263 & 22620.120 \\
\hline STORY18 & B1 & WIND & 72.000 & 249.263 & 18133.380 \\
\hline STORY18 & $\mathrm{B} 1$ & WIND & 72.000 & 248.297 & 17962.370 \\
\hline STORY18 & B1 & WIND & 96.000 & 248.297 & 12003.250 \\
\hline STORY18 & B1 & WIND & 120.000 & 248.297 & 6044.123 \\
\hline STORY18 & $\mathrm{B} 1$ & WIND & 144.000 & 248.297 & 85.001 \\
\hline STORY18 & $\mathrm{B} 1$ & WIND & 144.000 & 248.297 & -85.001 \\
\hline STORY18 & $\mathrm{B} 1$ & WIND & 168.000 & 248.297 & -6044.123 \\
\hline STORY18 & B1 & WIND & 192.000 & 248.297 & -12003.250 \\
\hline STORY18 & $\mathrm{B} 1$ & WIND & 216.000 & 248.297 & -17962.370 \\
\hline STORY18 & $\mathrm{B} 1$ & WIND & 216.000 & 249.263 & -18133.380 \\
\hline STORY18 & $\mathrm{B} 1$ & WIND & 234.000 & 249.263 & -22620.120 \\
\hline STORY 18 & $\mathrm{~B} 1$ & WIND & 252.000 & 249.263 & -27106.860 \\
\hline STORY18 & $\mathrm{B} 1$ & WIND & 270.000 & 249.263 & -31593.590 \\
\hline STORY17 & $\mathrm{B} 1$ & DEAD & 18.000 & -25.986 & -540.311 \\
\hline STORY17 & $\mathrm{B} 1$ & DEAD & 36.000 & -23.877 & -91.550 \\
\hline STORY17 & $\mathrm{B} 1$ & DEAD & 54.000 & -21.767 & 319.246 \\
\hline STORY 17 & B1 & DEAD & 72.000 & $\begin{array}{l}-19.658 \\
\end{array}$ & 692.075 \\
\hline STORY17 & B1 & DEAD & 72.000 & -13.209 & 707.815 \\
\hline STORY17 & B1 & DEAD & 96.000 & -10.397 & 991.081 \\
\hline STORY 17 & B1 & DEAD & 120.000 & -7.584 & 1206.852 \\
\hline STORY17 & B1 & DEAD & 144.000 & -4.772 & 1355.126 \\
\hline STORY17 & B1 & DEAD & 144.000 & 4.772 & 1355.126 \\
\hline STORY17 & $\mathrm{B} 1$ & DEAD & 168.000 & 7.584 & 1206.852 \\
\hline STORY17 & $\mathrm{B} 1$ & DEAD & 192.000 & 10.397 & 991.081 \\
\hline STORY17 & $\mathrm{B} 1$ & DEAD & 216.000 & 13.209 & 707.815 \\
\hline STORY17 & $\mathrm{B} 1$ & DEAD & 216.000 & 19.658 & 692.075 \\
\hline STORY17 & $\mathrm{B} 1$ & DEAD & 234.000 & 21.767 & 319.246 \\
\hline
\end{tabular}




\begin{tabular}{|c|c|c|c|c|c|}
\hline STORY17 & B1 & DEAD & 252.000 & 23.877 & -91.550 \\
\hline STORY17 & $\mathrm{B} 1$ & DEAD & 270.000 & 25.986 & -540.311 \\
\hline STORY 17 & B1 & LIVE & 18.000 & -5.434 & -110.456 \\
\hline STORY17 & $\mathrm{B} 1$ & LIVE & 36.000 & -5.434 & -12.641 \\
\hline STORY 17 & $\mathrm{~B} 1$ & LIVE & 54.000 & -5.434 & 85.175 \\
\hline STORY 17 & B1 & LIVE & 72.000 & -5.434 & 182.991 \\
\hline STORY17 & $\mathrm{B} 1$ & LIVE & 72.000 & -2.337 & 187.836 \\
\hline STORY17 & $\mathrm{B} 1$ & LIVE & 96.000 & -2.337 & 243.913 \\
\hline STORY17 & $\mathrm{B} 1$ & LIVE & 120.000 & -2.337 & 299.990 \\
\hline STORY17 & $\mathrm{B} 1$ & LIVE & 144.000 & -2.337 & 356.067 \\
\hline STORY17 & $\mathrm{B} 1$ & LIVE & 144.000 & 2.337 & 356.067 \\
\hline STORY17 & B1 & LIVE & 168.000 & 2.337 & 299.990 \\
\hline STORY17 & $\mathrm{B} 1$ & LIVE & 192.000 & 2.337 & 243.913 \\
\hline STORY17 & $\mathrm{B} 1$ & LIVE & 216.000 & 2.337 & 187.836 \\
\hline STORY17 & B1 & LIVE & 216.000 & 5.434 & 182.991 \\
\hline STORY17 & B1 & LIVE & 234.000 & 5.434 & 85.175 \\
\hline STORY17 & B1 & LIVE & 252.000 & 5.434 & -12.641 \\
\hline STORY17 & $\mathrm{B} 1$ & LIVE & 270.000 & 5.434 & -110.456 \\
\hline STORY17 & B1 & WIND & 18.000 & 269.198 & 34.121 .090 \\
\hline STORY17 & $\mathrm{B} 1$ & WIND & 36.000 & 269.198 & 29275.530 \\
\hline STORY17 & B1 & WIND & 54.000 & 269.198 & 24429.960 \\
\hline STORY17 & $\mathrm{B} 1$ & WIND & 72.000 & 269.198 & 19584.390 \\
\hline STORY17 & B1 & WIND & 72.000 & 268.165 & 19399.690 \\
\hline STORY17 & B1 & WIND & 96.000 & 268.165 & 12963.720 \\
\hline STORY17 & $\mathrm{B} 1$ & WIND & 120.000 & 268.165 & 6527.764 \\
\hline STORY17 & B1 & WIND & 144.000 & 268.165 & 91.804 \\
\hline STORY17 & B1 & WIND & 144.000 & 268.165 & -91.804 \\
\hline STORY17 & B1 & WIND & 168.000 & 268.165 & -6527.764 \\
\hline STORY17 & $\mathrm{B} 1$ & WIND & 192.000 & 268.165 & -12963.720 \\
\hline STORY17 & B1 & WIND & 216.000 & 268.165 & -19399.690 \\
\hline STORY17 & $\mathrm{B} 1$ & WIND & 216.000 & 269.198 & -19584.390 \\
\hline STORY17 & $\mathrm{B} 1$ & WIND & 234.000 & 269.198 & -24429.960 \\
\hline STORY17 & $\mathrm{B} 1$ & WIND & 252.000 & 269.198 & -29275.530 \\
\hline STORY17 & $\mathrm{B} 1$ & WIND & 270.000 & 269.198 & -34121.090 \\
\hline STORY16 & $\mathrm{B} 1$ & DEAD & 20.000 & -25.753 & -561.211 \\
\hline STORY16 & $\mathrm{B} 1$ & DEAD & 37.333 & -23.722 & -132.429 \\
\hline STORY16 & $\bar{B} 1$ & DEAD & 54.667 & -21.691 & 261.148 \\
\hline STORY 16 & $\mathrm{~B} 1$ & DEAD & 72.000 & -19.660 & 619.518 \\
\hline STORY 16 & $\mathrm{~B} 1$ & DEAD & 72.000 & -13.209 & 635.258 \\
\hline STORY16 & $\mathrm{B} 1$ & DEAD & 96.000 & -10.397 & 918.526 \\
\hline STORY16 & $\mathrm{B} 1$ & DEAD & 120.000 & -7.584 & 1134.299 \\
\hline STORY16 & $\mathrm{B} 1$ & DEAD & 144.000 & -4.772 & 1282.576 \\
\hline STORY16 & $\mathrm{B} 1$ & DEAD & 144.000 & 4.772 & 1282.576 \\
\hline STORY 16 & $\mathrm{~B} 1$ & DEAD & 168.000 & 7.584 & 1134.299 \\
\hline STORY 16 & B1 & DEAD & 192.000 & 10.397 & 918.526 \\
\hline STORY 16 & B1 & DEAD & 216.000 & $\begin{array}{l}13.209 \\
\end{array}$ & 635.258 \\
\hline STORY16 & $\bar{B} 1$ & DEAD & 216.000 & 19.660 & 619.518 \\
\hline
\end{tabular}




\begin{tabular}{|c|c|c|c|c|c|}
\hline STORY16 & $\mathrm{B} 1$ & DEAD & 233.333 & 21.691 & 261.148 \\
\hline STORY16 & B1 & DEAD & 250.667 & 23.722 & -132.429 \\
\hline STORY16 & B1 & DEAD & 268.000 & 25.753 & -561.211 \\
\hline STORY16 & B1 & LIVE & 20.000 & -5.435 & -119.102 \\
\hline STORY16 & B1 & LIVE & 37.333 & -5.435 & -24.902 \\
\hline STORY16 & B1 & LIVE & 54.667 & -5.435 & 69.298 \\
\hline STORY16 & B1 & LIVE & 72.000 & -5.435 & 163.498 \\
\hline STORY 16 & $\mathrm{~B} 1$ & LIVE & 72.000 & -2.337 & 168.343 \\
\hline STORY16 & B1 & LIVE & 96.000 & -2.337 & 224.421 \\
\hline STORY16 & B1 & LIVE & 120.000 & -2.337 & 280.498 \\
\hline STORY16 & B1 & LIVE & 144.000 & -2.337 & 336.576 \\
\hline STORY16 & B1 & LIVE & 144.000 & 2.337 & 336.576 \\
\hline STORY16 & B1 & LIVE & 168.000 & 2.337 & 280.498 \\
\hline STORY16 & B1 & LIVE & 192.000 & 2.337 & 224.421 \\
\hline STORY16 & B1 & LIVE & 216.000 & 2.337 & 168.343 \\
\hline STORY16 & B1 & LIVE & 216.000 & 5.435 & 163.498 \\
\hline STORY16 & B1 & LIVE & 233.333 & 5.435 & 69.298 \\
\hline STORY16 & B1 & LIVE & 250.667 & 5.435 & -24.902 \\
\hline STORY16 & $\mathrm{B} 1$ & LIVE & 268.000 & 5.435 & -119.102 \\
\hline STORY16 & B1 & WIND & 20.000 & 286.760 & 35774.600 \\
\hline STORY16 & B1 & WIND & 37.333 & 286.760 & 30804.090 \\
\hline STORY16 & B1 & WIND & 54.667 & 286.760 & 25833.590 \\
\hline STORY16 & B1 & WIND & 72.000 & 286.760 & 20863.080 \\
\hline STORY16 & B1 & WIND & 72.000 & 285.674 & 20666.310 \\
\hline STORY16 & B1 & WIND & 96.000 & 285.674 & 13810.140 \\
\hline STORY16 & B1 & WIND & 120.000 & 285.674 & 6953.968 \\
\hline STORY16 & B1 & WIND & 144.000 & 285.674 & 97.799 \\
\hline STORY16 & B1 & WIND & 144.000 & 285.674 & -97.799 \\
\hline STORY16 & $\mathrm{B} 1$ & WIND & 168.000 & 285.674 & -6953.968 \\
\hline STORY16 & B1 & WIND & 192.000 & 285.674 & -13810.140 \\
\hline STORY 16 & B1 & WIND & 216.000 & 285.674 & -20666.310 \\
\hline STORY16 & B1 & WIND & 216.000 & 286.760 & -20863.080 \\
\hline STORY 16 & B1 & WIND & 233.333 & 286.760 & -25833.590 \\
\hline STORY 16 & $\mathrm{~B} 1$ & WIND & 250.667 & 286.760 & -30804.090 \\
\hline STORY16 & B1 & WIND & 268.000 & 286.760 & -35774.600 \\
\hline STORY15 & B1 & DEAD & 20.000 & -25.755 & -632.120 \\
\hline STORY15 & $\mathrm{B} 1$ & DEAD & 37.333 & -23.723 & -203.311 \\
\hline STORY15 & B1 & DEAD & 54.667 & -21.692 & 190.292 \\
\hline STORY 15 & B1 & DEAD & 72.000 & -19.661 & 548.689 \\
\hline STORY15 & B1 & DEAD & 72.000 & -13.209 & 564.427 \\
\hline STORY15 & $\mathrm{B} 1$ & DEAD & 96.000 & -10.397 & 847.698 \\
\hline STORY15 & $\mathrm{B} 1$ & DEAD & 120.000 & -7.584 & 1063.473 \\
\hline STORY15 & $\mathrm{B} 1$ & DEAD & 144.000 & -4.772 & 1211.753 \\
\hline STORY15 & $\mathrm{B} 1$ & DEAD & 144.000 & 4.772 & 1211.753 \\
\hline STORY 15 & B1 & DEAD & 168.000 & 7.584 & 1063.473 \\
\hline STORY 15 & $\mathrm{~B} 1$ & DEAD & 192.000 & 10.397 & 847.698 \\
\hline STORY15 & $\mathrm{B} 1$ & DEAD & 216.000 & 13.209 & 564.427 \\
\hline
\end{tabular}




\begin{tabular}{|c|c|c|c|c|c|}
\hline STORY15 & $\mathrm{B} 1$ & DEAD & 216.000 & 19.661 & 548.689 \\
\hline STORY 15 & $\mathrm{~B} 1$ & DEAD & 233.333 & 21.692 & 190.292 \\
\hline STORY15 & B1 & DEAD & 250.667 & 23.723 & -203.311 \\
\hline STORY15 & B1 & DEAD & 268.000 & 25.755 & -632.120 \\
\hline STORY 15 & $\mathrm{~B} 1$ & LIVE & 20.000 & -5.435 & -138.152 \\
\hline STORY 15 & B1 & LIVE & 37.333 & -5.435 & -43.944 \\
\hline STORY15 & B1 & LIVE & 54.667 & -5.435 & 50.263 \\
\hline STORY15 & B1 & LIVE & 72.000 & -5.435 & 144.470 \\
\hline STORY15 & B1 & LIVE & 72.000 & -2.337 & 149.315 \\
\hline STORY15 & B1 & LIVE & 96.000 & -2.337 & 205.393 \\
\hline STORY15 & B1 & LIVE & 120.000 & -2.337 & 261.471 \\
\hline STORY15 & B1 & LIVE & 144.000 & -2.337 & 317.549 \\
\hline STORY15 & B1 & LIVE & 144.000 & 2.337 & 317.549 \\
\hline STORY15 & B1 & LIVE & 168.000 & 2.337 & 261.471 \\
\hline STORY15 & $\mathrm{B} 1$ & LIVE & 192.000 & 2.337 & 205.393 \\
\hline STORY15 & $\mathrm{B} 1$ & LIVE & 216.000 & 2.337 & 149.315 \\
\hline STORY15 & B1 & LIVE & 216.000 & 5.435 & 144.470 \\
\hline STORY15 & $\mathrm{B} 1$ & LIVE & 233.333 & 5.435 & 50.263 \\
\hline STORY15 & $\mathrm{B} 1$ & LIVE & 250.667 & 5.435 & -43.944 \\
\hline STORY15 & $\mathrm{B} 1$ & LIVE & 268.000 & 5.435 & -138.152 \\
\hline STORY15 & B1 & WIND & 20.000 & 306.491 & 38237.220 \\
\hline STORY15 & $\mathrm{B} 1$ & WIND & 37.333 & 306.491 & 32924.700 \\
\hline STORY15 & $\mathrm{B} 1$ & WIND & 54.667 & 306.491 & 27612.190 \\
\hline STORY15 & B1 & WIND & 72.000 & 306.491 & 22299.680 \\
\hline STORY15 & B1 & WIND & 72.000 & 305.345 & 22089.350 \\
\hline STORY15 & $\mathrm{B} 1$ & WIND & 96.000 & 305.345 & 14761.080 \\
\hline STORY15 & B1 & WIND & 120.000 & 305.345 & 7432.805 \\
\hline STORY15 & $\mathrm{B} 1$ & WIND & 144.000 & 305.345 & 104.535 \\
\hline STORY15 & B1 & WIND & 144.000 & 305.345 & -104.535 \\
\hline STORY15 & B1 & WIND & 168.000 & 305.345 & -7432.805 \\
\hline STORY15 & B1 & WIND & 192.000 & 305.345 & -14761.080 \\
\hline STORY15 & $\mathrm{B} 1$ & WIND & 216.000 & 305.345 & -22089.350 \\
\hline STORY15 & B1 & WIND & 216.000 & 306.491 & -22299.680 \\
\hline STORY 15 & $\mathrm{~B} 1$ & WIND & 233.333 & 306.491 & -27612.190 \\
\hline STORY15 & B1 & WIND & 250.667 & 306.491 & -32924.700 \\
\hline STORY 15 & $\mathrm{~B} 1$ & WIND & 268.000 & 306.491 & -38237.220 \\
\hline STORY14 & B1 & DEAD & 20.000 & -25.754 & -607.882 \\
\hline STORY 14 & B1 & DEAD & 37.333 & -23.723 & -179.082 \\
\hline STORY14 & $\mathrm{B} 1$ & DEAD & 54.667 & -21.692 & 214.512 \\
\hline STORY14 & $\mathrm{B} 1$ & DEAD & 72.000 & -19.661 & 572.900 \\
\hline STORY14 & $\mathrm{B} 1$ & DEAD & 72.000 & -13.209 & 588.639 \\
\hline STORY14 & $\mathrm{B} 1$ & DEAD & 96.000 & -10.397 & 871.909 \\
\hline STORY14 & $\mathrm{B} 1$ & DEAD & 120.000 & -7.584 & 1087.683 \\
\hline STORY 14 & B1 & DEAD & 144.000 & -4.772 & 1235.962 \\
\hline STORY14 & B1 & DEAD & 144.000 & 4.772 & 1235.962 \\
\hline STORY14 & B1 & DEAD & 168.000 & 7.584 & 1087.683 \\
\hline STORY14 & B1 & DEAD & 192.000 & 10.397 & 871.909 \\
\hline
\end{tabular}




\begin{tabular}{|c|c|c|c|c|c|}
\hline STORY 14 & $\mathrm{~B} 1$ & DEAD & 216.000 & 13.209 & 588.639 \\
\hline STORY14 & $\mathrm{B} 1$ & DEAD & 216.000 & 19.661 & 572.900 \\
\hline STORY 14 & $\mathrm{~B} 1$ & DEAD & 233.333 & 21.692 & 214.512 \\
\hline STORY14 & $\mathrm{B} 1$ & DEAD & 250.667 & 23.723 & -179.082 \\
\hline STORY14 & $\mathrm{B} 1$ & DEAD & 268.000 & 25.754 & -607.882 \\
\hline STORY 14 & B1 & LIVE & 20.000 & -5.435 & -131.641 \\
\hline STORY14 & $\mathrm{B} 1$ & LIVE & 37.333 & -5.435 & -37.436 \\
\hline STORY 14 & $\mathrm{~B} 1$ & LIVE & 54.667 & -5.435 & 56.769 \\
\hline STORY14 & $\mathrm{B} 1$ & LIVE & 72.000 & -5.435 & 150.974 \\
\hline STORY 14 & $\mathrm{~B} 1$ & LIVE & 72.000 & -2.337 & 155.818 \\
\hline STORY14 & $\mathrm{B} 1$ & LIVE & 96.000 & -2.337 & 211.896 \\
\hline STORY 14 & $\mathrm{~B} 1$ & LIVE & 120.000 & -2.337 & 267.974 \\
\hline STORY14 & $\mathrm{B} 1$ & LIVE & 144.000 & -2.337 & 324.052 \\
\hline STORY14 & $\mathrm{B} 1$ & LIVE & 144.000 & 2.337 & 324.052 \\
\hline STORY14 & $\mathrm{B} 1$ & LIVE & 168.000 & 2.337 & 267.974 \\
\hline STORY14 & $\mathrm{B} 1$ & LIVE & 192.000 & 2.337 & 211.896 \\
\hline STORY14 & $\mathrm{B} 1$ & LIVE & 216.000 & 2.337 & 155.818 \\
\hline STORY14 & $\mathrm{B} 1$ & LIVE & 216.000 & 5.435 & 150.974 \\
\hline STORY14 & B1 & LIVE & 233.333 & 5.435 & 56.769 \\
\hline STORY14 & $\mathrm{B} 1$ & LIVE & 250.667 & 5.435 & -37.436 \\
\hline STORY14 & B1 & LIVE & 268.000 & 5.435 & -131.641 \\
\hline STORY14 & $\mathrm{B} 1$ & WIND & 20.000 & 324.777 & 40518.630 \\
\hline STORY14 & $\mathrm{B} 1$ & WIND & 37.333 & 324.777 & 34889.160 \\
\hline STORY14 & $\mathrm{B} 1$ & WIND & 54.667 & 324.777 & 29259.680 \\
\hline STORY14 & B1 & WIND & 72.000 & 324.777 & 23630.210 \\
\hline STORY14 & $\mathrm{B} 1$ & WIND & 72.000 & 323.563 & 23407.330 \\
\hline STORY14 & $\mathrm{B} 1$ & WIND & 96.000 & 323.563 & 15641.810 \\
\hline STORY14 & $\mathrm{B} 1$ & WIND & 120.000 & 323.563 & 7876.292 \\
\hline STORY14 & $\mathrm{B} 1$ & WIND & 144.000 & 323.563 & 110.772 \\
\hline STORY14 & $\mathrm{B} 1$ & WIND & 144.000 & 323.563 & -110.772 \\
\hline STORY14 & $\mathrm{B} 1$ & WIND & 168.000 & 323.563 & -7876.292 \\
\hline STORY14 & $\mathrm{B} 1$ & WIND & 192.000 & 323.563 & -15641.810 \\
\hline STORY14 & B1 & WIND & 216.000 & 323.563 & -23407.330 \\
\hline STORY14 & B1 & WIND & 216.000 & 324.777 & -23630.210 \\
\hline STORY14 & B1 & WIND & 233.333 & 324.777 & -29259.680 \\
\hline STORY14 & B1 & WIND & 250.667 & 324.777 & -34889.160 \\
\hline STORY14 & B1 & WIND & 268.000 & 324.777 & -40518.630 \\
\hline STORY13 & $\mathrm{B} 1$ & DEAD & 22.000 & -25.521 & -623.414 \\
\hline STORY13 & B1 & DEAD & 38.667 & -23.568 & -214.338 \\
\hline STORY 13 & $\mathrm{~B} 1$ & DEAD & 55.333 & -21.615 & 162.189 \\
\hline STORY13 & $\mathrm{B} 1$ & DEAD & 72.000 & -19.662 & 506.165 \\
\hline STORY13 & B1 & DEAD & 72.000 & -13.209 & 521.903 \\
\hline STORY13 & B1 & DEAD & 96.000 & -10.397 & 805.175 \\
\hline STORY13 & $\mathrm{B} 1$ & DEAD & 120.000 & -7.585 & 1020.952 \\
\hline STORY13 & B1 & DEAD & 144.000 & -4.772 & 1169.233 \\
\hline STORY 13 & B1 & DEAD & 144.000 & 4.772 & 1169.233 \\
\hline STORY13 & B1 & DEAD & 168.000 & 7.585 & 1020.952 \\
\hline
\end{tabular}




\begin{tabular}{|c|c|c|c|c|c|}
\hline STORY13 & $\mathrm{B} 1$ & DEAD & 192.000 & 10.397 & 805.175 \\
\hline STORY13 & $\mathrm{B} 1$ & DEAD & 216.000 & 13.209 & 521.903 \\
\hline STORY13 & B1 & DEAD & 216.000 & 19.662 & 506.165 \\
\hline STORY13 & B1 & DEAD & 232.667 & 21.615 & 162.189 \\
\hline STORY13 & $\mathrm{B} 1$ & DEAD & 249.333 & 23.568 & -214.338 \\
\hline STORY13 & $\mathrm{B} 1$ & DEAD & 266.000 & 25.521 & -623.414 \\
\hline STORY13 & B1 & LIVE & 22.000 & -5.435 & -138.714 \\
\hline STORY13 & $\mathrm{B} 1$ & LIVE & 38.667 & -5.435 & -48.126 \\
\hline STORY13 & B1 & LIVE & 55.333 & -5.435 & 42.462 \\
\hline STORY13 & B1 & LIVE & 72.000 & -5.435 & 133.049 \\
\hline STORY13 & B1 & LIVE & 72.000 & -2.337 & 137.894 \\
\hline STORY13 & B1 & LIVE & 96.000 & -2.337 & 193.973 \\
\hline STORY13 & $\mathrm{B} 1$ & LIVE & 120.000 & -2.337 & 250.051 \\
\hline STORY13 & $\mathrm{B} 1$ & LIVE & 144.000 & -2.337 & 306.130 \\
\hline STORY13 & B1 & LIVE & 144.000 & 2.337 & 306.130 \\
\hline STORY13 & $\mathrm{B} 1$ & LIVE & 168.000 & 2.337 & 250.051 \\
\hline STORY13 & B1 & LIVE & 192.000 & 2.337 & 193.973 \\
\hline STORY13 & $\mathrm{B} 1$ & LIVE & 216.000 & 2.337 & 137.894 \\
\hline STORY13 & $\mathrm{B} 1$ & LIVE & 216.000 & 5.435 & 133.049 \\
\hline STORY13 & B1 & LIVE & 232.667 & 5.435 & 42.462 \\
\hline STORY13 & $\mathrm{B} 1$ & LIVE & 249.333 & 5.435 & -48.126 \\
\hline STORY13 & B1 & LIVE & 266.000 & 5.435 & -138.714 \\
\hline STORY13 & $\mathrm{B} 1$ & WIND & 22.000 & 340.337 & 41780.710 \\
\hline STORY13 & $\mathrm{B} 1$ & WIND & 38.667 & 340.337 & 36108.420 \\
\hline STORY13 & B1 & WIND & 55.333 & 340.337 & 30436.130 \\
\hline STORY13 & B1 & WIND & 72.000 & 340.337 & 24763.840 \\
\hline STORY13 & $\mathrm{B} 1$ & WIND & 72.000 & 339.086 & 24530.280 \\
\hline STORY13 & B1 & WIND & 96.000 & 339.086 & 16392.210 \\
\hline STORY13 & B1 & WIND & 120.000 & 339.086 & 8254.150 \\
\hline STORY13 & B1 & WIND & 144.000 & 339.086 & 116.088 \\
\hline STORY13 & $\mathrm{B} 1$ & WIND & 144.000 & 339.086 & -116.088 \\
\hline STORY13 & $\mathrm{B} 1$ & WIND & 168.000 & 339.086 & -8254.150 \\
\hline STORY13 & $\mathrm{B} 1$ & WIND & 192.000 & 339.086 & -16392.210 \\
\hline STORY13 & B1 & WIND & 216.000 & 339.086 & -24530.280 \\
\hline STORY13 & B1 & WIND & 216.000 & 340.337 & -24763.840 \\
\hline STORY13 & $\mathrm{B} 1$ & WIND & 232.667 & 340.337 & -30436.130 \\
\hline STORY13 & B1 & WIND & 249.333 & 340.337 & -36108.420 \\
\hline STORY13 & B1 & WIND & 266.000 & 340.337 & -41780.710 \\
\hline STORY12 & $\mathrm{B} 1$ & DEAD & 22.000 & -25.522 & -661.694 \\
\hline STORY12 & $\mathrm{B} 1$ & DEAD & 38.667 & -23.569 & -252.605 \\
\hline STORY 12 & B1 & DEAD & 55.333 & -21.616 & 123.935 \\
\hline STORY12 & B1 & DEAD & 72.000 & -19.663 & 467.925 \\
\hline STORY 12 & $\mathrm{~B} 1$ & DEAD & 72.000 & -13.209 & 483.662 \\
\hline STORY12 & $\mathrm{B} 1$ & DEAD & 96.000 & -10.397 & 766.935 \\
\hline STORY12 & $\mathrm{B} 1$ & DEAD & 120.000 & -7.585 & 982.713 \\
\hline STORY 12 & B1 & DEAD & 144.000 & -4.772 & 1130.996 \\
\hline STORY12 & B1 & DEAD & 144.000 & 4.772 & 1130.996 \\
\hline
\end{tabular}




\begin{tabular}{|c|c|c|c|c|c|}
\hline STORY12 & $\mathrm{B} 1$ & DEAD & 168.000 & 7.585 & 982.713 \\
\hline STORY 12 & B1 & DEAD & 192.000 & 10.397 & 766.935 \\
\hline STORY12 & B1 & DEAD & 216.000 & 13.209 & 483.662 \\
\hline STORY12 & $\mathrm{B} 1$ & DEAD & 216.000 & 19.663 & 467.925 \\
\hline STORY12 & $\mathrm{B} 1$ & DEAD & 232.667 & 21.616 & 123.935 \\
\hline STORY12 & $\mathrm{B} 1$ & DEAD & 249.333 & 23.569 & -252.605 \\
\hline STORY12 & $\mathrm{B} 1$ & DEAD & 266.000 & 25.522 & -661.694 \\
\hline STORY 12 & B1 & LIVE & 22.000 & -5.435 & -149.019 \\
\hline STORY12 & $\mathrm{B} 1$ & LIVE & 38.667 & -5.435 & -58.427 \\
\hline STORY12 & B1 & LIVE & 55.333 & -5.435 & 32.164 \\
\hline STORY12 & $\mathrm{B} 1$ & LIVE & 72.000 & -5.435 & 122.756 \\
\hline STORY12 & $\mathrm{B} 1$ & LIVE & 72.000 & -2.337 & 127.600 \\
\hline STORY12 & $\mathrm{B} 1$ & LIVE & 96.000 & -2.337 & 183.679 \\
\hline STORY 12 & $\mathrm{~B} 1$ & LIVE & 120.000 & -2.337 & 239.758 \\
\hline STORY12 & $\mathrm{B} 1$ & LIVE & 144.000 & -2.337 & 295.837 \\
\hline STORY12 & $\mathrm{B} 1$ & LIVE & 144.000 & 2.337 & 295.837 \\
\hline STORY12 & B1 & LIVE & 168.000 & 2.337 & 239.758 \\
\hline STORY12 & $\mathrm{B} 1$ & LIVE & 192.000 & 2.337 & 183.679 \\
\hline STORY 12 & $\mathrm{~B} 1$ & LIVE & 216.000 & 2.337 & 127.600 \\
\hline STORY12 & $\mathrm{B} 1$ & LIVE & 216.000 & 5.435 & 122.756 \\
\hline STORY 12 & $\mathrm{~B} 1$ & LIVE & 232.667 & 5.435 & 32.164 \\
\hline STORY 12 & $\mathrm{~B} 1$ & LIVE & 249.333 & 5.435 & -58.427 \\
\hline STORY12 & $\mathrm{B} 1$ & LIVE & 266.000 & 5.435 & -149.019 \\
\hline STORY12 & $\mathrm{B} 1$ & WIND & 22.000 & 359.159 & 44092.550 \\
\hline STORY12 & $\mathrm{B} 1$ & WIND & 38.667 & 359.159 & 38106.560 \\
\hline STORY12 & $\mathrm{B} 1$ & WIND & 55.333 & 359.159 & 32120.570 \\
\hline STORY12 & $\mathrm{B} 1$ & WIND & 72.000 & 359.159 & 26134.590 \\
\hline STORY12 & $\mathrm{B} 1$ & WIND & 72.000 & 357.855 & 25888.090 \\
\hline STORY12 & $\mathrm{B} 1$ & WIND & 96.000 & 357.855 & 17299.570 \\
\hline STORY12 & $\mathrm{B} 1$ & WIND & 120.000 & 357.855 & 8711.040 \\
\hline STORY12 & B1 & WIND & 144.000 & 357.855 & 122.515 \\
\hline STORY12 & $\mathrm{B} 1$ & WIND & 144.000 & 357.855 & -122.515 \\
\hline STORY12 & $\mathrm{B} 1$ & WIND & 168.000 & 357.855 & -8711.040 \\
\hline STORY12 & $\mathrm{B} 1$ & WIND & 192.000 & 357.855 & -17299.570 \\
\hline STORY 12 & B1 & WIND & 216.000 & 357.855 & -25888.090 \\
\hline STORY12 & $\mathrm{B} 1$ & WIND & 216.000 & 359.159 & -26134.590 \\
\hline STORY12 & B1 & WIND & 232.667 & 359.159 & -32120.570 \\
\hline STORY12 & $\mathrm{B} 1$ & WIND & 249.333 & 359.159 & -38106.560 \\
\hline STORY12 & B1 & WIND & 266.000 & 359.159 & -44092.550 \\
\hline STORY11 & $\mathrm{B} 1$ & DEAD & 24.000 & -25.288 & -648.352 \\
\hline STORY11 & $\mathrm{B} 1$ & DEAD & 48.000 & -22.476 & -75.178 \\
\hline STORY11 & $\mathrm{B} 1$ & DEAD & 72.000 & -19.664 & 430.501 \\
\hline STORY11 & $\mathrm{B} 1$ & DEAD & 72.000 & -13.209 & 446.237 \\
\hline STORY11 & $\mathrm{B} 1$ & DEAD & 96.000 & -10.397 & 729.513 \\
\hline STORY 11 & $\mathrm{~B} 1$ & DEAD & 120.000 & -7.585 & 945.293 \\
\hline STORY11 & $\mathrm{B} 1$ & DEAD & 144.000 & -4.772 & 1093.578 \\
\hline STORY11 & $\mathrm{B} 1$ & DEAD & 144.000 & 4.772 & 1093.578 \\
\hline
\end{tabular}




\begin{tabular}{|c|c|c|c|c|c|}
\hline STORY11 & B1 & DEAD & 168.000 & 7.585 & 945.293 \\
\hline STORY11 & B1 & DEAD & 192.000 & 10.397 & 729.513 \\
\hline STORY11 & B1 & DEAD & 216.000 & 13.209 & 446.237 \\
\hline STORY11 & B1 & DEAD & 216.000 & 19.664 & 430.501 \\
\hline STORY11 & $\mathrm{B1}$ & DEAD & 240.000 & 22.476 & -75.178 \\
\hline STORY11 & B1 & DEAD & 264.000 & 25.288 & -648.352 \\
\hline STORY11 & $\mathrm{B} 1$ & LIVE & 24.000 & -5.436 & -148.108 \\
\hline STORY11 & $\mathrm{B} 1$ & LIVE & 48.000 & -5.436 & -17.652 \\
\hline STORY11 & $\mathrm{B} 1$ & LIVE & 72.000 & -5.436 & 112.805 \\
\hline STORY11 & $\mathrm{B} 1$ & LIVE & 72.000 & -2.337 & 117.649 \\
\hline STORY11 & $\mathrm{B} 1$ & LIVE & 96.000 & -2.337 & 173.728 \\
\hline STORY11 & B1 & LIVE & 120.000 & -2.337 & 229.807 \\
\hline STORY11 & $\mathrm{B} 1$ & LIVE & 144.000 & -2.337 & 285.886 \\
\hline STORY11 & B1 & LIVE & 144.000 & 2.337 & 285.886 \\
\hline STORY11 & $\mathrm{B} 1$ & LIVE & 168.000 & 2.337 & 229.807 \\
\hline STORY11 & $\mathrm{B} 1$ & LIVE & 192.000 & 2.337 & 173.728 \\
\hline STORY11 & B1 & LIVE & 216.000 & 2.337 & 117.649 \\
\hline STORY11 & $\mathrm{B} 1$ & LIVE & 216.000 & 5.436 & 112.805 \\
\hline STORY11 & B1 & LIVE & 240.000 & 5.436 & -17.652 \\
\hline STORY11 & $\mathrm{B} 1$ & LIVE & 264.000 & 5.436 & -148.108 \\
\hline STORY11 & B1 & WIND & 24.000 & 372.825 & 45026.040 \\
\hline STORY11 & $\mathrm{B} 1$ & WIND & 48.000 & 372.825 & 36078.250 \\
\hline STORY11 & $\mathrm{B} 1$ & WIND & 72.000 & 372.825 & 27130.460 \\
\hline STORY11 & B1 & WIND & 72.000 & 371.491 & 26874.570 \\
\hline STORY11 & B1 & WIND & 96.000 & 371.491 & 17958.770 \\
\hline STORY11 & $\mathrm{B} 1$ & WIND & 120.000 & 371.491 & 9042.979 \\
\hline STORY11 & $\mathrm{B} 1$ & WIND & 144.000 & 371.491 & 127.185 \\
\hline STORY11 & $\mathrm{B} 1$ & WIND & 144.000 & 371.491 & -127.185 \\
\hline STORY11 & B1 & WIND & 168.000 & 371.491 & -9042.979 \\
\hline STORY11 & $\mathrm{B} 1$ & WIND & 192.000 & 371.491 & -17958.770 \\
\hline STORY11 & B1 & WIND & 216.000 & 371.491 & -26874.570 \\
\hline STORY11 & $\mathrm{B} 1$ & WIND & 216.000 & 372.825 & -27130.460 \\
\hline STORY11 & B1 & WIND & 240.000 & 372.825 & -36078.250 \\
\hline STORY11 & $\mathrm{B} 1$ & WIND & 264.000 & 372.825 & -45026.040 \\
\hline STORY10 & $\mathrm{B} 1$ & DEAD & 24.000 & -25.288 & -676.319 \\
\hline STORY10 & B1 & DEAD & 48.000 & -22.476 & -103.146 \\
\hline STORY10 & $\mathrm{B} 1$ & DEAD & 72.000 & -19.664 & 402.531 \\
\hline STORY10 & $\mathrm{B} 1$ & DEAD & 72.000 & -13.209 & 418.267 \\
\hline STORY 10 & B1 & DEAD & 96.000 & -10.397 & 701.539 \\
\hline STORY10 & B1 & DEAD & 120.000 & -7.585 & 917.315 \\
\hline STORY10 & B1 & DEAD & 144.000 & -4.772 & 1065.595 \\
\hline STORY10 & B1 & DEAD & 144.000 & 4.772 & 1065.595 \\
\hline STORY 10 & B1 & DEAD & 168.000 & 7.585 & 917.315 \\
\hline STORY 10 & B1 & DEAD & 192.000 & 10.397 & 701.539 \\
\hline STORY10 & B1 & DEAD & 216.000 & 13.209 & 418.267 \\
\hline STORY10 & $\mathrm{B} 1$ & DEAD & 216.000 & 19.664 & 402.531 \\
\hline STORY10 & $\mathrm{B} 1$ & DEAD & 240.000 & 22.476 & -103.146 \\
\hline
\end{tabular}




\begin{tabular}{|c|c|c|c|c|c|}
\hline STORY10 & $\mathrm{B} 1$ & DEAD & 264.000 & 25.288 & -676.319 \\
\hline STORY 10 & B1 & LIVE & 24.000 & -5.436 & -156.096 \\
\hline STORY 10 & B1 & LIVE & 48.000 & -5.436 & -25.635 \\
\hline STORY10 & B1 & LIVE & 72.000 & -5.436 & 104.827 \\
\hline STORY10 & B1 & LIVE & 72.000 & -2.337 & 109.671 \\
\hline STORY10 & $\mathrm{B} 1$ & LIVE & 96.000 & -2.337 & 165.751 \\
\hline STORY10 & B1 & LIVE & 120.000 & -2.337 & 221.831 \\
\hline STORY 10 & B1 & LIVE & 144.000 & -2.337 & 277.910 \\
\hline STORY 10 & $\mathrm{~B} 1$ & LIVE & 144.000 & 2.337 & 277.910 \\
\hline STORY10 & $\mathrm{B} 1$ & LIVE & 168.000 & 2.337 & 221.831 \\
\hline STORY10 & B1 & LIVE & 192.000 & 2.337 & 165.751 \\
\hline STORY10 & B1 & LIVE & 216.000 & 2.337 & 109.671 \\
\hline STORY10 & B1 & LIVE & 216.000 & 5.436 & 104.827 \\
\hline STORY10 & $\mathrm{B} 1$ & LIVE & 240.000 & 5.436 & -25.635 \\
\hline STORY10 & B1 & LIVE & 264.000 & 5.436 & -156.096 \\
\hline STORY 10 & $\mathrm{~B} 1$ & WIND & 24.000 & 386.105 & 46633.440 \\
\hline STORY10 & B1 & WIND & 48.000 & 386.105 & 37366.920 \\
\hline STORY10 & B1 & WIND & 72.000 & 386.105 & 28100.400 \\
\hline STORY10 & $\mathrm{B} 1$ & WIND & 72.000 & 384.773 & 27835.370 \\
\hline STORY10 & $\mathrm{B} 1$ & WIND & 96.000 & 384.773 & 18600.820 \\
\hline STORY10 & B1 & WIND & 120.000 & 384.773 & 9366.279 \\
\hline STORY10 & $\mathrm{B} 1$ & WIND & 144.000 & 384.773 & 131.735 \\
\hline STORY 10 & $\mathrm{~B} 1$ & WIND & 144.000 & 384.773 & -131.735 \\
\hline STORY10 & $\mathrm{B} 1$ & WIND & 168.000 & 384.773 & -9366.279 \\
\hline STORY10 & $\mathrm{B} 1$ & WIND & 192.000 & 384.773 & -18600.820 \\
\hline STORY10 & $\mathrm{B} 1$ & WIND & 216.000 & 384.773 & -27835.370 \\
\hline STORY10 & $\mathrm{B} 1$ & WIND & 216.000 & 386.105 & -28100.400 \\
\hline STORY10 & B1 & WIND & 240.000 & 386.105 & -37366.920 \\
\hline STORY10 & $\mathrm{B} 1$ & WIND & 264.000 & 386.105 & -46633.440 \\
\hline STORY9 & $\mathrm{B} 1$ & DEAD & 26.000 & -25.057 & -656.413 \\
\hline STORY9 & B1 & DEAD & 49.000 & -22.362 & -111.099 \\
\hline STORY9 & B1 & DEAD & 72.000 & -19.667 & 372.228 \\
\hline STORY9 & $\mathrm{B} 1$ & DEAD & 72.000 & -13.210 & 387.962 \\
\hline STORY9 & $\mathrm{B} 1$ & DEAD & 96.000 & -10.398 & 671.252 \\
\hline STORY9 & $\mathrm{B} 1$ & DEAD & 120.000 & -7.585 & 887.046 \\
\hline STORY9 & $\mathrm{B} 1$ & DEAD & 144.000 & -4.773 & 1035.345 \\
\hline STORY9 & B1 & DEAD & 144.000 & 4.773 & 1035.345 \\
\hline STORY9 & $\mathrm{B} 1$ & DEAD & 168.000 & 7.585 & 887.046 \\
\hline STORY9 & $\mathrm{B} 1$ & DEAD & 192.000 & 10.398 & 671.252 \\
\hline STORY9 & B1 & DEAD & 216.000 & 13.210 & 387.962 \\
\hline STORY9 & $\mathrm{B} 1$ & DEAD & 216.000 & 19.667 & 372.228 \\
\hline STORY9 & $\mathrm{B} 1$ & DEAD & 239.000 & 22.362 & -111.099 \\
\hline STORY9 & $\mathrm{B} 1$ & DEAD & 262.000 & 25.057 & -656.413 \\
\hline STORY9 & $\mathrm{B} 1$ & LIVE & 26.000 & -5.435 & -151.118 \\
\hline STORY9 & $\mathrm{B} 1$ & LIVE & 49.000 & -5.435 & -26.102 \\
\hline STORY9 & $\mathrm{B} 1$ & LIVE & 72.000 & -5.435 & 98.913 \\
\hline STORY9 & $\mathrm{B} 1$ & LIVE & 72.000 & -2.336 & 103.757 \\
\hline
\end{tabular}




\begin{tabular}{|c|c|c|c|c|c|}
\hline STORY9 & B1 & LIVE & 96.000 & -2.336 & 159.833 \\
\hline STORY9 & B1 & LIVE & 120.000 & -2.336 & 215.909 \\
\hline STORY9 & B1 & LIVE & 144.000 & -2.336 & 271.984 \\
\hline STORY9 & B1 & LIVE & 144.000 & 2.336 & 271.984 \\
\hline STORY9 & B1 & LIVE & 168.000 & 2.336 & 215.909 \\
\hline STORY9 & $\mathrm{B} 1$ & LIVE & 192.000 & 2.336 & 159.833 \\
\hline STORY9 & B1 & LIVE & 216.000 & 2.336 & 103.757 \\
\hline STORY9 & B1 & LIVE & 216.000 & 5.435 & 98.913 \\
\hline STORY9 & B1 & LIVE & 239.000 & 5.435 & -26.102 \\
\hline STORY9 & B1 & LIVE & 262.000 & 5.435 & -151.118 \\
\hline STORY9 & $\mathrm{B} 1$ & WIND & 26.000 & 376.664 & 44740.680 \\
\hline STORY9 & $\mathrm{B} 1$ & WIND & 49.000 & 376.664 & 36077.400 \\
\hline STORY9 & $\mathrm{B} 1$ & WIND & 72.000 & 376.664 & 27414.120 \\
\hline STORY9 & B1 & WIND & 72.000 & 375.373 & 27155.390 \\
\hline STORY9 & $\mathrm{B} 1$ & WIND & 96.000 & 375.373 & 18146.430 \\
\hline STORY9 & B1 & WIND & 120.000 & 375.373 & 9137.472 \\
\hline STORY9 & B1 & WIND & 144.000 & 375.373 & 128.513 \\
\hline STORY9 & $\mathrm{B} 1$ & WIND & 144.000 & 375.373 & -128.513 \\
\hline STORY9 & $\mathrm{B} 1$ & WIND & 168.000 & 375.373 & -9137.472 \\
\hline STORY9 & B1 & WIND & 192.000 & 375.373 & -18146.430 \\
\hline STORY9 & $\mathrm{B} 1$ & WIND & 216.000 & 375.373 & -27155.390 \\
\hline STORY9 & B1 & WIND & 216.000 & 376.664 & -27414.120 \\
\hline STORY9 & $\mathrm{B} 1$ & WIND & 239.000 & 376.664 & -36077.400 \\
\hline STORY9 & B1 & WIND & 262.000 & 376.664 & -44740.680 \\
\hline STORY8 & $\mathrm{B} 1$ & DEAD & 26.000 & -31.519 & -684.123 \\
\hline STORY8 & B1 & DEAD & 49.000 & -27.686 & -3.260 \\
\hline STORY 8 & $\mathrm{~B} 1$ & DEAD & 72.000 & -23.853 & 589.442 \\
\hline STORY8 & $\mathrm{B} 1$ & DEAD & 72.000 & -17.017 & 603.592 \\
\hline STORY8 & $\mathrm{B} 1$ & DEAD & 96.000 & -13.017 & 964.005 \\
\hline STORY8 & $\mathrm{B} 1$ & DEAD & 120.000 & -9.018 & 1228.424 \\
\hline STORY 8 & $\mathrm{~B} 1$ & DEAD & 144.000 & -5.018 & 1396.849 \\
\hline STORY8 & $\mathrm{B} 1$ & DEAD & 144.000 & 5.018 & 1396.849 \\
\hline STORY8 & B1 & DEAD & 168.000 & 9.018 & 1228.424 \\
\hline STORY8 & $\mathrm{B} 1$ & DEAD & 192.000 & 13.017 & 964.005 \\
\hline STORY 8 & $\mathrm{~B} 1$ & DEAD & 216.000 & 17.017 & 603.592 \\
\hline STORY8 & B1 & DEAD & 216.000 & 23.853 & 589.442 \\
\hline STORY8 & $\mathrm{B} 1$ & DEAD & 239.000 & 27.686 & -3.260 \\
\hline STORY 8 & $\mathrm{~B} 1$ & DEAD & 262.000 & 31.519 & -684.123 \\
\hline STORY 8 & $\mathrm{~B} 1$ & LIVE & 26.000 & -5.692 & -132.785 \\
\hline STORY 8 & $\mathrm{~B} 1$ & LIVE & 49.000 & -5.692 & -1.879 \\
\hline STORY8 & B1 & LIVE & 72.000 & -5.692 & 129.027 \\
\hline STORY 8 & $\mathrm{~B} 1$ & LIVE & 72.000 & -2.433 & 133.041 \\
\hline STORY 8 & $\mathrm{~B} 1$ & LIVE & 96.000 & -2.433 & 191.436 \\
\hline STORY8 & $\mathrm{B} 1$ & LIVE & 120.000 & -2.433 & 249.830 \\
\hline STORY8 & $\mathrm{B} 1$ & LIVE & 144.000 & -2.433 & 308.225 \\
\hline STORY 8 & $\mathrm{~B} 1$ & LIVE & 144.000 & 2.433 & 308.225 \\
\hline STORY 8 & $\mathrm{~B} 1$ & LIVE & 168.000 & 2.433 & 249.830 \\
\hline
\end{tabular}




\begin{tabular}{|c|c|c|c|c|c|}
\hline STORY8 & $\mathrm{B} 1$ & LIVE & 192.000 & 2.433 & 191.436 \\
\hline STORY8 & B1 & LIVE & 216.000 & 2.433 & 133.041 \\
\hline STORY8 & $\mathrm{B} 1$ & LIVE & 216.000 & 5.692 & 129.027 \\
\hline STORY8 & $\mathrm{B} 1$ & LIVE & 239.000 & 5.692 & -1.879 \\
\hline STORY8 & $\mathrm{B} 1$ & LIVE & 262.000 & 5.692 & -132.785 \\
\hline STORY8 & $\mathrm{B} 1$ & WIND & 26.000 & 510.578 & 60698.910 \\
\hline STORY8 & $\mathrm{B} 1$ & WIND & 49.000 & 510.578 & 48955.630 \\
\hline STORY8 & B1 & WIND & 72.000 & 510.578 & 37212.340 \\
\hline STORY8 & $\mathrm{B} 1$ & WIND & 72.000 & 511.625 & 36961.210 \\
\hline STORY8 & $\mathrm{B} 1$ & WIND & 96.000 & 511.625 & 24682.200 \\
\hline STORY8 & $\mathrm{B} 1$ & WIND & 120.000 & 511.625 & 12403.190 \\
\hline STORY8 & $\mathrm{B} 1$ & WIND & 144.000 & 511.625 & 124.187 \\
\hline STORY8 & $\mathrm{B} 1$ & WIND & 144.000 & 511.625 & -124.187 \\
\hline STORY8 & $\mathrm{B} 1$ & WIND & 168.000 & 511.625 & -12403.190 \\
\hline STORY8 & $\mathrm{B} 1$ & WIND & 192.000 & 511.625 & -24682.200 \\
\hline STORY8 & $\mathrm{B} 1$ & WIND & 216.000 & 511.625 & -36961.210 \\
\hline STORY8 & $\mathrm{B} 1$ & WIND & 216.000 & 510.578 & -37212.340 \\
\hline STORY8 & $\mathrm{B} 1$ & WIND & 239.000 & 510.578 & -48955.630 \\
\hline STORY8 & $\mathrm{B} 1$ & WIND & 262.000 & 510.578 & -60698.910 \\
\hline STORY7 & $\mathrm{B} 1$ & DEAD & 27.000 & -31.337 & -668.140 \\
\hline STORY7 & $\mathrm{B} 1$ & DEAD & 49.500 & -27.588 & -5.235 \\
\hline STORY7 & $\mathrm{B} 1$ & DEAD & 72.000 & -23.838 & 573.301 \\
\hline STORY7 & $\mathrm{B} 1$ & DEAD & 72.000 & -17.006 & 587.432 \\
\hline STORY7 & $\mathrm{B} 1$ & DEAD & 96.000 & -13.007 & 947.584 \\
\hline STORY7 & $\mathrm{B} 1$ & DEAD & 120.000 & -9.007 & 1211.743 \\
\hline STORY7 & $\mathrm{B} 1$ & DEAD & 144.000 & -5.007 & 1379.908 \\
\hline STORY7 & $\mathrm{B} 1$ & DEAD & 144.000 & 5.007 & 1379.908 \\
\hline STORY7 & $\mathrm{B} 1$ & DEAD & 168.000 & 9.007 & 1211.743 \\
\hline STORY7 & $\mathrm{B} 1$ & DEAD & 192.000 & 13.007 & 947.584 \\
\hline STORY7 & $\mathrm{B} 1$ & DEAD & 216.000 & 17.006 & 587.432 \\
\hline STORY7 & $\mathrm{B} 1$ & DEAD & 216.000 & 23.838 & 573.301 \\
\hline STORY7 & $\mathrm{B} 1$ & DEAD & 238.500 & 27.588 & -5.235 \\
\hline STORY7 & $\mathrm{B} 1$ & DEAD & 261.000 & 31.337 & -668.140 \\
\hline STORY7 & $\mathrm{B} 1$ & LIVE & 27.000 & -5.678 & -127.161 \\
\hline STORY7 & $\mathrm{B} 1$ & LIVE & 49.500 & -5.678 & 0.589 \\
\hline STORY7 & $\mathrm{B} 1$ & LIVE & 72.000 & -5.678 & 128.338 \\
\hline STORY7 & $\mathrm{B} 1$ & LIVE & 72.000 & -2.426 & 132.344 \\
\hline STORY7 & $\mathrm{B} 1$ & LIVE & 96.000 & -2.426 & 190.571 \\
\hline STORY7 & $\mathrm{B} 1$ & LIVE & 120.000 & -2.426 & 248.797 \\
\hline STORY7 & $\mathrm{B} 1$ & LIVE & 144.000 & -2.426 & 307.024 \\
\hline STORY7 & $\mathrm{B} 1$ & LIVE & 144.000 & 2.426 & 307.024 \\
\hline STORY7 & $\mathrm{B} 1$ & LIVE & 168.000 & 2.426 & 248.797 \\
\hline STORY7 & $\mathrm{B} 1$ & LIVE & 192.000 & 2.426 & 190.571 \\
\hline STORY7 & $\mathrm{B} 1$ & LIVE & 216.000 & 2.426 & 132.344 \\
\hline STORY7 & $\mathrm{B} 1$ & LIVE & 216.000 & 5.678 & 128.338 \\
\hline STORY7 & $\mathrm{B} 1$ & LIVE & 238.500 & 5.678 & 0.589 \\
\hline STORY7 & $\mathrm{B} 1$ & LIVE & 261.000 & 5.678 & -127.161 \\
\hline
\end{tabular}




\begin{tabular}{|c|c|c|c|c|c|}
\hline STORY7 & B1 & WIND & 27.000 & 469.117 & 55211.840 \\
\hline STORY7 & B1 & WIND & 49.500 & 469.117 & 44656.720 \\
\hline STORY7 & B1 & WIND & 72.000 & 469.117 & 34101.600 \\
\hline STORY7 & $\mathrm{B} 1$ & WIND & 72.000 & 468.877 & 33872.560 \\
\hline STORY7 & B1 & WIND & 96.000 & 468.877 & 22619.510 \\
\hline STORY7 & $\mathrm{B} 1$ & WIND & 120.000 & 468.877 & 11366.460 \\
\hline STORY7 & B1 & WIND & 144.000 & 468.877 & 113.414 \\
\hline STORY7 & $\mathrm{B} 1$ & WIND & 144.000 & $\begin{aligned} 468.877 \\
\end{aligned}$ & -113.414 \\
\hline STORY7 & $\mathrm{B} 1$ & WIND & 168.000 & 468.877 & -11366.460 \\
\hline STORY7 & B1 & WIND & 192.000 & 468.877 & -22619.510 \\
\hline STORY7 & B1 & WIND & 216.000 & 468.877 & -33872.560 \\
\hline STORY7 & $\mathrm{B} 1$ & WIND & 216.000 & 469.117 & -34101.600 \\
\hline STORY7 & $\mathrm{B} 1$ & WIND & 238.500 & 469.117 & -44656.720 \\
\hline STORY7 & $\mathrm{B} 1$ & WIND & 261.000 & 469.117 & -55211.840 \\
\hline STORY6 & $\mathrm{B} 1$ & DEAD & 29.000 & -31.004 & -665.650 \\
\hline STORY6 & $\mathrm{B} 1$ & DEAD & 50.500 & -27.421 & -37.579 \\
\hline STORY6 & $\mathrm{B} 1$ & DEAD & 72.000 & -23.838 & 513.455 \\
\hline STORY6 & $\mathrm{B} 1$ & DEAD & 72.000 & -17.006 & 527.585 \\
\hline STORY6 & $\mathrm{B} 1$ & DEAD & 96.000 & -13.006 & 887.733 \\
\hline STORY6 & $\mathrm{B} 1$ & DEAD & 120.000 & -9.007 & 1151.887 \\
\hline STORY6 & $\mathrm{B} 1$ & DEAD & 144.000 & -5.007 & 1320.048 \\
\hline STORY6 & $\mathrm{B} 1$ & DEAD & 144.000 & 5.007 & 1320.048 \\
\hline STORY6 & $\mathrm{B} 1$ & DEAD & 168.000 & 9.007 & 1151.887 \\
\hline STORY6 & $\mathrm{B} 1$ & DEAD & 192.000 & 13.006 & 887.733 \\
\hline STORY6 & $\mathrm{B} 1$ & DEAD & 216.000 & 17.006 & 527.585 \\
\hline STORY6 & $\mathrm{B} 1$ & DEAD & 216.000 & 23.838 & 513.455 \\
\hline STORY6 & $\mathrm{B} 1$ & DEAD & 237.500 & 27.421 & -37.579 \\
\hline STORY6 & $\mathrm{B} 1$ & DEAD & 259.000 & 31.004 & -665.650 \\
\hline STORY6 & $\mathrm{B} 1$ & LIVE & 29.000 & -5.679 & -130.045 \\
\hline STORY6 & B1 & LIVE & 50.500 & -5.679 & -7.956 \\
\hline STORY6 & $\mathrm{B} 1$ & LIVE & 72.000 & -5.679 & 114.134 \\
\hline STORY6 & $\mathrm{B} 1$ & LIVE & 72.000 & -2.426 & 118.140 \\
\hline STORY6 & $\mathrm{B} 1$ & LIVE & 96.000 & -2.426 & 176.372 \\
\hline STORY6 & $\mathrm{B} 1$ & LIVE & 120.000 & -2.426 & 234.603 \\
\hline STORY6 & $\mathrm{B} 1$ & LIVE & 144.000 & -2.426 & 292.835 \\
\hline STORY6 & $\mathrm{B} 1$ & LIVE & 144.000 & 2.426 & 292.835 \\
\hline STORY6 & B1 & LIVE & 168.000 & 2.426 & 234.603 \\
\hline STORY6 & $\mathrm{B} 1$ & LIVE & 192.000 & 2.426 & 176.372 \\
\hline STORY6 & $\mathrm{B} 1$ & LIVE & 216.000 & 2.426 & 118.140 \\
\hline STORY6 & $\mathrm{B} 1$ & LIVE & 216.000 & 5.679 & 114.134 \\
\hline STORY6 & B1 & LIVE & 237.500 & 5.679 & -7.956 \\
\hline STORY6 & B1 & LIVE & 259.000 & 5.679 & -130.045 \\
\hline STORY6 & B1 & WIND & 29.000 & 463.723 & 53650.040 \\
\hline STORY6 & $\mathrm{B} 1$ & WIND & 50.500 & 463.723 & 43680.010 \\
\hline STORY6 & $\mathrm{B} 1$ & WIND & 72.000 & 463.723 & 33709.970 \\
\hline STORY 6 & $\mathrm{~B} 1$ & WIND & 72.000 & 463.494 & 33483.700 \\
\hline STORY6 & $\mathrm{B} 1$ & WIND & 96.000 & 463.494 & 22359.840 \\
\hline
\end{tabular}




\begin{tabular}{|c|c|c|c|c|c|}
\hline STORY6 & $\mathrm{B} 1$ & WIND & 120.000 & 463.494 & 11235.980 \\
\hline STORY6 & $\mathrm{B} 1$ & WIND & 144.000 & 463.494 & 112.116 \\
\hline STORY6 & $\mathrm{B} 1$ & WIND & 144.000 & 463.494 & -112.116 \\
\hline STORY6 & $\mathrm{B} 1$ & WIND & 168.000 & 463.494 & -11235.980 \\
\hline STORY6 & $\mathrm{B} 1$ & WIND & 192.000 & 463.494 & -22359.840 \\
\hline STORY6 & $\mathrm{B} 1$ & WIND & 216.000 & 463.494 & -33483.700 \\
\hline STORY6 & $\mathrm{B} 1$ & WIND & 216.000 & 463.723 & -33709.970 \\
\hline STORY6 & $\mathrm{B} 1$ & WIND & 237.500 & 463.723 & -43680.010 \\
\hline STORY6 & $\mathrm{B} 1$ & WIND & 259.000 & 463.723 & -53650.040 \\
\hline STORY5 & $\mathrm{B} 1$ & DEAD & 30.000 & -30.839 & -675.880 \\
\hline STORY5 & $\mathrm{B} 1$ & DEAD & 51.000 & -27.339 & -65.005 \\
\hline STORY5 & $\mathrm{B} 1$ & DEAD & 72.000 & -23.840 & 472.375 \\
\hline STORY 5 & $\mathrm{~B} 1$ & DEAD & 72.000 & -17.006 & 486.503 \\
\hline STORY5 & $\mathrm{B} 1$ & DEAD & 96.000 & -13.007 & 846.661 \\
\hline STORY5 & $\mathrm{B} 1$ & DEAD & 120.000 & -9.007 & 1110.824 \\
\hline STORY5 & $\mathrm{B} 1$ & DEAD & 144.000 & -5.007 & 1278.994 \\
\hline STORY5 & $\mathrm{B} 1$ & DEAD & 144.000 & 5.007 & 1278.994 \\
\hline STORY5 & $\mathrm{B} 1$ & DEAD & 168.000 & 9.007 & 1110.824 \\
\hline STORY5 & B1 & DEAD & 192.000 & 13.007 & 846.661 \\
\hline STORY5 & $\mathrm{B} 1$ & DEAD & 216.000 & 17.006 & 486.503 \\
\hline STORY5 & $\mathrm{B} 1$ & DEAD & 216.000 & 23.840 & 472.375 \\
\hline STORY5 & $\mathrm{B} 1$ & DEAD & 237.000 & 27.339 & -65.005 \\
\hline STORY5 & $\mathrm{B} 1$ & DEAD & 258.000 & 30.839 & -675.880 \\
\hline STORY5 & $\mathrm{B} 1$ & LIVE & 30.000 & -5.679 & -133.232 \\
\hline STORY5 & $\mathrm{B} 1$ & LIVE & 51.000 & -5.679 & -13.977 \\
\hline STORY5 & $\mathrm{B} 1$ & LIVE & 72.000 & -5.679 & 105.279 \\
\hline STORY5 & $\mathrm{B} 1$ & LIVE & 72.000 & -2.426 & 109.285 \\
\hline STORY5 & $\mathrm{B} 1$ & LIVE & 96.000 & -2.426 & 167.517 \\
\hline STORY5 & $\mathrm{B} 1$ & LIVE & 120.000 & -2.426 & 225.750 \\
\hline STORY5 & $\mathrm{B} 1$ & LIVE & 144.000 & -2.426 & 283.982 \\
\hline STORY5 & $\mathrm{B} 1$ & LIVE & 144.000 & 2.426 & 283.982 \\
\hline STORY5 & $\mathrm{B} 1$ & LIVE & 168.000 & 2.426 & 225.750 \\
\hline STORY5 & B1 & LIVE & 192.000 & 2.426 & 167.517 \\
\hline STORY5 & B1 & LIVE & 216.000 & 2.426 & 109.285 \\
\hline STORY5 & $\mathrm{B} 1$ & LIVE & 216.000 & 5.679 & 105.279 \\
\hline STORY5 & $\mathrm{B} 1$ & LIVE & 237.000 & 5.679 & -13.977 \\
\hline STORY5 & $\mathrm{B} 1$ & LIVE & 258.000 & 5.679 & -133.232 \\
\hline STORY5 & B1 & WIND & 30.000 & 490.258 & 56226.950 \\
\hline STORY5 & $\mathrm{B} 1$ & WIND & 51.000 & 490.258 & 45931.520 \\
\hline STORY5 & $\mathrm{B} 1$ & WIND & 72.000 & 490.258 & 35636.100 \\
\hline STORY5 & $\mathrm{B} 1$ & WIND & 72.000 & 489.977 & 35396.870 \\
\hline STORY 5 & $\mathrm{~B} 1$ & WIND & 96.000 & 489.977 & 23637.420 \\
\hline STORY5 & B1 & WIND & 120.000 & 489.977 & 11877.970 \\
\hline STORY5 & B1 & WIND & 144.000 & 489.977 & 118.525 \\
\hline STORY5 & $\mathrm{B} 1$ & WIND & 144.000 & 489.977 & -118.525 \\
\hline STORY5 & $\mathrm{B} 1$ & WIND & 168.000 & 489.977 & -11877.970 \\
\hline STORY 5 & $\mathrm{~B} 1$ & WIND & 192.000 & 489.977 & -23637.420 \\
\hline
\end{tabular}




\begin{tabular}{|c|c|c|c|c|c|}
\hline STORY5 & $\mathrm{B} 1$ & WIND & 216.000 & 489.977 & -35396.870 \\
\hline STORY5 & $\mathrm{B} 1$ & WIND & 216.000 & 490.258 & -35636.100 \\
\hline STORY5 & B1 & WIND & 237.000 & 490.258 & -45931.520 \\
\hline STORY5 & B1 & WIND & 258.000 & 490.258 & -56226.950 \\
\hline STORY4 & $\mathrm{B} 1$ & DEAD & 32.000 & -30.506 & -639.772 \\
\hline STORY4 & $\mathrm{B} 1$ & DEAD & 52.000 & -27.173 & -62.974 \\
\hline STORY4 & $\mathrm{B} 1$ & DEAD & 72.000 & -23.840 & 447.162 \\
\hline STORY4 & $\mathrm{B} 1$ & DEAD & 72.000 & -17.007 & 461.290 \\
\hline STORY4 & $\mathrm{B} 1$ & DEAD & 96.000 & -13.007 & 821.450 \\
\hline STORY4 & $\mathrm{B} 1$ & DEAD & 120.000 & -9.007 & 1085.615 \\
\hline STORY4 & $\mathrm{B} 1$ & DEAD & 144.000 & -5.007 & 1253.787 \\
\hline STORY4 & $\mathrm{B} 1$ & DEAD & 144.000 & 5.007 & 1253.787 \\
\hline STORY4 & $\mathrm{B} 1$ & DEAD & 168.000 & 9.007 & 1085.615 \\
\hline STORY4 & $\mathrm{B} 1$ & DEAD & 192.000 & 13.007 & 821.450 \\
\hline STORY4 & $\mathrm{B} 1$ & DEAD & 216.000 & 17.007 & 461.290 \\
\hline STORY4 & $\mathrm{B} 1$ & DEAD & 216.000 & 23.840 & 447.162 \\
\hline STORY4 & $\mathrm{B} 1$ & DEAD & 236.000 & 27.173 & -62.974 \\
\hline STORY4 & $\mathrm{B} 1$ & DEAD & 256.000 & 30.506 & -639.772 \\
\hline STORY4 & B1 & LIVE & 32.000 & -5.679 & -129.559 \\
\hline STORY4 & $\mathrm{B} 1$ & LIVE & 52.000 & -5.679 & -15.978 \\
\hline STORY4 & $\mathrm{B} 1$ & LIVE & 72.000 & -5.679 & 97.604 \\
\hline STORY4 & $\mathrm{B} 1$ & LIVE & 72.000 & -2.426 & 101.610 \\
\hline STORY4 & $\mathrm{B} 1$ & LIVE & 96.000 & -2.426 & 159.843 \\
\hline STORY4 & B1 & LIVE & 120.000 & -2.426 & 218.077 \\
\hline STORY4 & $\mathrm{B} 1$ & LIVE & 144.000 & -2.426 & 276.310 \\
\hline STORY4 & $\mathrm{B} 1$ & LIVE & 144.000 & 2.426 & 276.310 \\
\hline STORY4 & $\mathrm{B} 1$ & LIVE & 168.000 & 2.426 & 218.077 \\
\hline STORY4 & $\mathrm{B} 1$ & LIVE & 192.000 & 2.426 & 159.843 \\
\hline STORY4 & B1 & LIVE & 216.000 & 2.426 & 101.610 \\
\hline STORY4 & $\mathrm{B} 1$ & LIVE & 216.000 & 5.679 & 97.604 \\
\hline STORY4 & $\mathrm{B} 1$ & LIVE & 236.000 & 5.679 & -15.978 \\
\hline STORY4 & $\mathrm{B} 1$ & LIVE & 256.000 & 5.679 & -129.559 \\
\hline STORY4 & $\mathrm{B} 1$ & WIND & 32.000 & 551.593 & 62156.870 \\
\hline STORY4 & $\mathrm{B} 1$ & WIND & 52.000 & 551.593 & 51125.020 \\
\hline STORY4 & $\mathrm{B} 1$ & WIND & 72.000 & 551.593 & 40093.170 \\
\hline STORY4 & $\mathrm{B} 1$ & WIND & 72.000 & 551.259 & 39823.990 \\
\hline STORY4 & $\mathrm{B} 1$ & WIND & 96.000 & 551.259 & 26593.780 \\
\hline STORY4 & $\mathrm{B} 1$ & WIND & 120.000 & 551.259 & 13363.560 \\
\hline STORY4 & $\mathrm{B} 1$ & WIND & 144.000 & 551.259 & 133.350 \\
\hline STORY4 & $\mathrm{B} 1$ & WIND & 144.000 & 551.259 & -133.350 \\
\hline STORY4 & $\mathrm{B} 1$ & WIND & 168.000 & 551.259 & -13363.560 \\
\hline STORY4 & $\mathrm{B} 1$ & WIND & 192.000 & 551.259 & -26593.780 \\
\hline STORY4 & $\mathrm{B} 1$ & WIND & 216.000 & 551.259 & -39823.990 \\
\hline STORY4 & $\mathrm{B} 1$ & WIND & 216.000 & 551.593 & -40093.170 \\
\hline STORY4 & B1 & WIND & 236.000 & 551.593 & -51125.020 \\
\hline STORY4 & $\mathrm{B} 1$ & WIND & 256.000 & 551.593 & -62156.870 \\
\hline STORY3 & $\mathrm{B} 1$ & DEAD & 34.000 & -24.120 & -562.910 \\
\hline
\end{tabular}




\begin{tabular}{|c|c|c|c|c|c|}
\hline STORY3 & $\mathrm{B} 1$ & DEAD & 53.000 & -21.894 & -125.782 \\
\hline STORY3 & B1 & DEAD & 72.000 & -19.667 & 269.043 \\
\hline STORY3 & $\mathrm{B} 1$ & DEAD & 72.000 & -13.210 & 284.777 \\
\hline STORY3 & $\mathrm{B} 1$ & DEAD & 96.000 & -10.397 & 568.057 \\
\hline STORY3 & $\mathrm{B} 1$ & DEAD & 120.000 & -7.585 & 783.842 \\
\hline STORY3 & $\mathrm{B} 1$ & DEAD & 144.000 & -4.773 & 932.130 \\
\hline STORY3 & $\mathrm{B} 1$ & DEAD & 144.000 & 4.773 & 932.130 \\
\hline STORY3 & $\mathrm{B} 1$ & DEAD & 168.000 & 7.585 & 783.842 \\
\hline STORY3 & $\mathrm{B} 1$ & DEAD & 192.000 & 10.397 & 568.057 \\
\hline STORY3 & B1 & DEAD & 216.000 & 13.210 & 284.777 \\
\hline STORY3 & $\mathrm{B} 1$ & DEAD & 216.000 & 19.667 & 269.043 \\
\hline STORY3 & $\mathrm{B} 1$ & DEAD & 235.000 & 21.894 & -125.782 \\
\hline STORY3 & $\mathrm{B} 1$ & DEAD & 254.000 & 24.120 & -562.910 \\
\hline STORY3 & $\mathrm{B} 1$ & LIVE & 34.000 & -5.437 & -136.355 \\
\hline STORY3 & $\mathrm{B} 1$ & LIVE & 53.000 & -5.437 & -33.060 \\
\hline STORY3 & $\mathrm{B} 1$ & LIVE & 72.000 & -5.437 & 70.236 \\
\hline STORY3 & $\mathrm{B} 1$ & LIVE & 72.000 & -2.337 & 75.079 \\
\hline STORY3 & B1 & LIVE & 96.000 & -2.337 & 131.160 \\
\hline STORY3 & $\mathrm{B} 1$ & LIVE & 120.000 & -2.337 & 187.241 \\
\hline STORY3 & $\mathrm{B} 1$ & LIVE & 144.000 & -2.337 & 243.322 \\
\hline STORY3 & $\mathrm{B} 1$ & LIVE & 144.000 & 2.337 & 243.322 \\
\hline STORY3 & $\mathrm{B} 1$ & LIVE & 168.000 & 2.337 & 187.241 \\
\hline STORY3 & $\mathrm{B} 1$ & LIVE & 192.000 & 2.337 & 131.160 \\
\hline STORY3 & $\mathrm{B} 1$ & LIVE & 216.000 & 2.337 & 75.079 \\
\hline STORY3 & $\mathrm{B} 1$ & LIVE & 216.000 & 5.437 & 70.236 \\
\hline STORY3 & $\mathrm{B} 1$ & LIVE & 235.000 & 5.437 & -33.060 \\
\hline STORY3 & B1 & LIVE & 254.000 & 5.437 & -136.355 \\
\hline STORY3 & $\mathrm{B} 1$ & WIND & 34.000 & 380.152 & 42108.310 \\
\hline STORY3 & $\mathrm{B} 1$ & WIND & 53.000 & 380.152 & 34885.430 \\
\hline STORY3 & $\mathrm{B} 1$ & WIND & 72.000 & 380.152 & 27662.540 \\
\hline STORY3 & $\mathrm{B} 1$ & WIND & 72.000 & 378.776 & 27401.580 \\
\hline STORY3 & B1 & WIND & 96.000 & 378.776 & 18310.950 \\
\hline STORY3 & $\mathrm{B} 1$ & WIND & 120.000 & 378.776 & 9220.313 \\
\hline STORY3 & B1 & WIND & 144.000 & 378.776 & 129.680 \\
\hline STORY3 & $\mathrm{B} 1$ & WIND & 144.000 & 378.776 & -129.680 \\
\hline STORY3 & B1 & WIND & 168.000 & 378.776 & -9220.313 \\
\hline STORY3 & $\mathrm{B} 1$ & WIND & 192.000 & 378.776 & -18310.950 \\
\hline STORY3 & $\mathrm{B} 1$ & WIND & 216.000 & 378.776 & -27401.580 \\
\hline STORY3 & B1 & WIND & 216.000 & 380.152 & -27662.540 \\
\hline STORY3 & B1 & WIND & 235.000 & 380.152 & -34885.430 \\
\hline STORY3 & $\mathrm{B} 1$ & WIND & 254.000 & 380.152 & -42108.310 \\
\hline STORY2 & $\mathrm{B} 1$ & DEAD & 36.000 & -23.886 & -525.768 \\
\hline STORY2 & $\mathrm{B} 1$ & DEAD & 54.000 & -21.776 & -114.809 \\
\hline STORY2 & $\mathrm{B} 1$ & DEAD & 72.000 & -19.667 & 258.184 \\
\hline STORY 2 & $\mathrm{~B} 1$ & DEAD & 72.000 & -13.209 & 273.918 \\
\hline STORY2 & B1 & DEAD & 96.000 & -10.397 & 557.198 \\
\hline STORY2 & $\mathrm{B} 1$ & DEAD & 120.000 & -7.585 & 772.982 \\
\hline
\end{tabular}




\begin{tabular}{|c|c|c|c|c|c|}
\hline STORY2 & $\mathrm{B} 1$ & DEAD & 144.000 & -4.773 & 921.270 \\
\hline STORY2 & $\mathrm{B} 1$ & DEAD & 144.000 & 4.773 & 921.270 \\
\hline STORY2 & B1 & DEAD & 168.000 & 7.585 & 772.982 \\
\hline STORY2 & B1 & DEAD & 192.000 & 10.397 & 557.198 \\
\hline STORY2 & B1 & DEAD & 216.000 & 13.209 & 273.918 \\
\hline STORY2 & B1 & DEAD & 216.000 & 19.667 & 258.184 \\
\hline STORY2 & $\mathrm{B} 1$ & DEAD & 234.000 & 21.776 & -114.809 \\
\hline STORY2 & $\mathrm{B} 1$ & DEAD & 252.000 & 23.886 & -525.768 \\
\hline STORY2 & $\mathrm{B} 1$ & LIVE & 36.000 & -5.437 & -129.472 \\
\hline STORY2 & $\mathrm{B} 1$ & LIVE & 54.000 & -5.437 & -31.611 \\
\hline STORY2 & $\mathrm{B} 1$ & LIVE & 72.000 & -5.437 & 66.249 \\
\hline STORY2 & $\mathrm{B} 1$ & LIVE & 72.000 & -2.337 & 71.093 \\
\hline STORY2 & $\mathrm{B} 1$ & LIVE & 96.000 & -2.337 & 127.174 \\
\hline STORY2 & $\mathrm{B} 1$ & LIVE & 120.000 & -2.337 & 183.255 \\
\hline STORY2 & $\mathrm{B} 1$ & LIVE & 144.000 & -2.337 & 239.336 \\
\hline STORY2 & $\mathrm{B} 1$ & LIVE & 144.000 & 2.337 & 239.336 \\
\hline STORY2 & $\mathrm{B} 1$ & LIVE & 168.000 & 2.337 & 183.255 \\
\hline STORY2 & $\mathrm{B} 1$ & LIVE & 192.000 & 2.337 & 127.174 \\
\hline STORY2 & $\mathrm{B} 1$ & LIVE & 216.000 & 2.337 & 71.093 \\
\hline STORY2 & $\mathrm{B} 1$ & LIVE & 216.000 & 5.437 & 66.249 \\
\hline STORY2 & $\mathrm{B} 1$ & LIVE & 234.000 & 5.437 & -31.611 \\
\hline STORY2 & $\mathrm{B} 1$ & LIVE & 252.000 & 5.437 & -129.472 \\
\hline STORY2 & $\mathrm{B} 1$ & WIND & 36.000 & 361.789 & 39350.200 \\
\hline STORY2 & B1 & WIND & 54.000 & 361.789 & 32838.000 \\
\hline STORY2 & $\mathrm{B} 1$ & WIND & 72.000 & 361.789 & 26325.810 \\
\hline STORY2 & $\mathrm{B} 1$ & WIND & 72.000 & 360.473 & 26077.440 \\
\hline STORY2 & $\mathrm{B} 1$ & WIND & 96.000 & 360.473 & 17426.100 \\
\hline STORY2 & $\mathrm{B} 1$ & WIND & 120.000 & 360.473 & 8774.756 \\
\hline STORY2 & $\mathrm{B} 1$ & WIND & 144.000 & 360.473 & 123.413 \\
\hline STORY2 & $\mathrm{B} 1$ & WIND & 144.000 & 360.473 & -123.413 \\
\hline STORY2 & $\mathrm{B} 1$ & WIND & 168.000 & 360.473 & -8774.756 \\
\hline STORY2 & $\mathrm{B} 1$ & WIND & 192.000 & 360.473 & -17426.100 \\
\hline STORY2 & $\mathrm{B} 1$ & WIND & 216.000 & 360.473 & -26077.440 \\
\hline STORY2 & $\mathrm{B} 1$ & WIND & 216.000 & 361.789 & -26325.810 \\
\hline STORY2 & $\mathrm{B} 1$ & WIND & 234.000 & 361.789 & -32838.000 \\
\hline STORY2 & $\mathrm{B} 1$ & WIND & 252.000 & 361.789 & -39350.200 \\
\hline STORY1 & $\overline{\mathrm{B} 1}$ & DEAD & 38.000 & -23.651 & -477.852 \\
\hline STORY1 & $\mathrm{B} 1$ & DEAD & 55.000 & -21.659 & -92.711 \\
\hline STORY1 & $\mathrm{B} 1$ & DEAD & 72.000 & -19.667 & 258.565 \\
\hline STORY 1 & $\mathrm{~B} 1$ & DEAD & 72.000 & -13.210 & 274.299 \\
\hline STORY 1 & $\mathrm{~B} 1$ & DEAD & 96.000 & -10.397 & 557.579 \\
\hline STORY1 & $\mathrm{B} 1$ & DEAD & 120.000 & -7.585 & 773.364 \\
\hline STORY1 & $\mathrm{B} 1$ & DEAD & 144.000 & -4.773 & 921.653 \\
\hline STORY 1 & $\mathrm{~B} 1$ & DEAD & 144.000 & 4.773 & 921.653 \\
\hline STORY 1 & B1 & DEAD & 168.000 & 7.585 & 773.364 \\
\hline STORY1 & $\mathrm{B} 1$ & DEAD & 192.000 & 10.397 & $557.57 \overline{9}$ \\
\hline STORY 1 & $\mathrm{~B} 1$ & DEAD & 216.000 & 13.210 & 274.299 \\
\hline
\end{tabular}




\begin{tabular}{|c|c|c|c|c|c|}
\hline STORY1 & $\mathrm{B} 1$ & DEAD & 216.000 & 19.667 & 258.565 \\
\hline STORY1 & $\mathrm{B} 1$ & DEAD & 233.000 & 21.659 & -92.711 \\
\hline STORY1 & $\mathrm{B} 1$ & DEAD & 250.000 & 23.651 & -477.852 \\
\hline STORY1 & $\mathrm{B} 1$ & LIVE & 38.000 & -5.437 & -118.286 \\
\hline STORY1 & $\mathrm{B} 1$ & LIVE & 55.000 & -5.437 & -25.862 \\
\hline STORY1 & $\mathrm{B} 1$ & LIVE & 72.000 & -5.437 & 66.562 \\
\hline STORY 1 & $\mathrm{~B} 1$ & LIVE & 72.000 & -2.337 & 71.405 \\
\hline STORY1 & $\mathrm{B} 1$ & LIVE & 96.000 & -2.337 & 127.486 \\
\hline STORY1 & $\mathrm{B} 1$ & LIVE & 120.000 & -2.337 & 183.567 \\
\hline STORY1 & $\mathrm{B} 1$ & LIVE & 144.000 & -2.337 & 239.648 \\
\hline STORY1 & $\mathrm{B} 1$ & LIVE & 144.000 & 2.337 & 239.648 \\
\hline STORY1 & $\mathrm{B} 1$ & LIVE & 168.000 & 2.337 & 183.567 \\
\hline STORY1 & $\mathrm{B} 1$ & LIVE & 192.000 & 2.337 & 127.486 \\
\hline STORY1 & $\mathrm{B} 1$ & LIVE & 216.000 & 2.337 & 71.405 \\
\hline STORY1 & $\mathrm{B} 1$ & LIVE & 216.000 & 5.437 & 66.562 \\
\hline STORY1 & $\mathrm{B} 1$ & LIVE & 233.000 & 5.437 & -25.862 \\
\hline STORY1 & $\mathrm{B} 1$ & LIVE & 250.000 & 5.437 & -118.286 \\
\hline STORY1 & $\mathrm{B} 1$ & WIND & 38.000 & 247.255 & 26398.100 \\
\hline STORY1 & $\mathrm{B} 1$ & WIND & 55.000 & 247.255 & 22194.770 \\
\hline STORY 1 & B1 & WIND & 72.000 & 247.255 & 17991.440 \\
\hline STORY1 & $\mathrm{B} 1$ & WIND & 72.000 & 246.352 & 17821.700 \\
\hline STORY1 & $\mathrm{B} 1$ & WIND & 96.000 & 246.352 & 11909.250 \\
\hline STORY1 & $\mathrm{B} 1$ & WIND & 120.000 & 246.352 & 5996.794 \\
\hline STORY1 & $\mathrm{B} 1$ & WIND & 144.000 & 246.352 & 84.342 \\
\hline STORY1 & $\mathrm{B} 1$ & WIND & 144.000 & 246.352 & -84.342 \\
\hline STORY1 & $\mathrm{B} 1$ & WIND & 168.000 & 246.352 & -5996.794 \\
\hline STORY1 & $\mathrm{B} 1$ & WIND & 192.000 & 246.352 & -11909.250 \\
\hline STORY1 & B1 & WIND & 216.000 & 246.352 & -17821.700 \\
\hline STORY1 & B1 & WIND & 216.000 & 247.255 & -17991.440 \\
\hline STORY1 & $\mathrm{B} 1$ & WIND & 233.000 & 247.255 & -22194.770 \\
\hline STORY1 & $\mathrm{B} 1$ & WIND & 250.000 & 247.255 & -26398.100 \\
\hline STORY30 & B2 & DEAD & 9.000 & -27.014 & 435.544 \\
\hline STORY30 & B2 & DEAD & 30.000 & -24.554 & 977.009 \\
\hline STORY30 & B2 & DEAD & 51.000 & -22.093 & 1466.799 \\
\hline STORY30 & $\mathrm{B} 2$ & DEAD & 72.000 & -19.632 & 1904.911 \\
\hline STORY30 & B2 & DEAD & 72.000 & -13.207 & 1920.671 \\
\hline STORY30 & $\mathrm{B} 2$ & DEAD & 96.000 & -10.395 & 2203.894 \\
\hline STORY 30 & $\bar{B} 2$ & DEAD & 120.000 & -7.583 & 2419.622 \\
\hline STORY30 & B2 & DEAD & 144.000 & -4.770 & 2567.854 \\
\hline STORY30 & $\mathrm{B} 2$ & DEAD & 144.000 & 4.770 & 2567.854 \\
\hline STORY30 & $\mathrm{B} 2$ & DEAD & 168.000 & 7.583 & 2419.622 \\
\hline STORY30 & B2 & DEAD & 192.000 & 10.395 & 2203.894 \\
\hline STORY 30 & B2 & DEAD & 216.000 & 13.207 & 1920.671 \\
\hline STORY30 & B2 & DEAD & 216.000 & 19.632 & 1904.911 \\
\hline STORY 30 & $\mathrm{~B} 2$ & DEAD & 237.000 & 22.093 & 1466.799 \\
\hline STORY 30 & $\mathrm{~B} 2$ & DEAD & 258.000 & 24.554 & 977.009 \\
\hline STORY30 & B2 & DEAD & 279.000 & 27.014 & 435.544 \\
\hline
\end{tabular}




\begin{tabular}{|c|c|c|c|c|c|}
\hline STORY30 & B2 & LIVE & 9.000 & -5.427 & 166.905 \\
\hline STORY30 & B2 & LIVE & 30.000 & -5.427 & 280.877 \\
\hline STORY30 & B2 & LIVE & 51.000 & -5.427 & 394.849 \\
\hline STORY30 & B2 & LIVE & 72.000 & -5.427 & 508.820 \\
\hline STORY30 & $\mathrm{B} 2$ & LIVE & 72.000 & -2.336 & 513.671 \\
\hline STORY30 & B2 & LIVE & 96.000 & -2.336 & $569.7 \overline{36}$ \\
\hline STORY30 & $\mathrm{B} 2$ & LIVE & 120.000 & -2.336 & 625.802 \\
\hline STORY30 & B2 & LIVE & 144.000 & -2.336 & 681.867 \\
\hline STORY30 & $\mathrm{B} 2$ & LIVE & 144.000 & 2.336 & 681.867 \\
\hline STORY30 & B2 & LIVE & 168.000 & 2.336 & 625.802 \\
\hline STORY30 & $\mathrm{B} 2$ & LIVE & 192.000 & 2.336 & 569.736 \\
\hline STORY30 & B2 & LIVE & 216.000 & 2.336 & 513.671 \\
\hline STORY30 & $\mathrm{B} 2$ & LIVE & 216.000 & 5.427 & 508.820 \\
\hline STORY30 & $\mathrm{B} 2$ & LIVE & 237.000 & 5.427 & 394.849 \\
\hline STORY30 & B2 & LIVE & 258.000 & 5.427 & 280.877 \\
\hline STORY30 & B2 & LIVE & 279.000 & 5.427 & 166.905 \\
\hline STORY30 & $\mathrm{B} 2$ & WIND & 9.000 & 5.592 & 758.953 \\
\hline STORY30 & $\mathrm{B} 2$ & WIND & 30.000 & 5.592 & 641.513 \\
\hline STORY30 & $\mathrm{B} 2$ & WIND & 51.000 & 5.592 & 524.073 \\
\hline STORY30 & $\mathrm{B} 2$ & WIND & 72.000 & 5.592 & 406.633 \\
\hline STORY30 & B2 & WIND & 72.000 & 5.568 & 402.800 \\
\hline STORY30 & $\mathrm{B} 2$ & WIND & 96.000 & 5.568 & 269.169 \\
\hline STORY30 & B2 & WIND & 120.000 & 5.568 & 135.537 \\
\hline STORY30 & B2 & WIND & 144.000 & 5.568 & 1.906 \\
\hline STORY30 & B2 & WIND & 144.000 & 5.568 & -1.906 \\
\hline STORY30 & B2 & WIND & 168.000 & 5.568 & -135.537 \\
\hline STORY30 & $\mathrm{B} 2$ & WIND & 192.000 & 5.568 & -269.169 \\
\hline STORY30 & B2 & WIND & 216.000 & 5.568 & -402.800 \\
\hline STORY30 & B2 & WIND & 216.000 & 5.592 & -406.633 \\
\hline STORY30 & B2 & WIND & 237.000 & 5.592 & -524.073 \\
\hline STORY30 & B2 & WIND & 258.000 & 5.592 & -641.513 \\
\hline STORY30 & B2 & WIND & 279.000 & 5.592 & -758.953 \\
\hline STORY29 & B2 & DEAD & 9.000 & -27.016 & 350.007 \\
\hline STORY29 & $\mathrm{B} 2$ & DEAD & 30.000 & -24.556 & 891.511 \\
\hline STORY29 & $\mathrm{B} 2$ & DEAD & 51.000 & -22.095 & 1381.339 \\
\hline STORY29 & B2 & DEAD & 72.000 & -19.634 & 1819.490 \\
\hline STORY29 & B2 & DEAD & 72.000 & -13.207 & 1835.248 \\
\hline STORY29 & $\mathrm{B} 2$ & DEAD & 96.000 & -10.395 & 2118.475 \\
\hline STORY29 & $\mathrm{B} 2$ & DEAD & 120.000 & -7.583 & 2334.206 \\
\hline STORY29 & $\mathrm{B} 2$ & DEAD & 144.000 & -4.770 & 2482.441 \\
\hline STORY29 & B2 & DEAD & 144.000 & 4.770 & 2482.441 \\
\hline STORY29 & B2 & DEAD & 168.000 & 7.583 & 2334.206 \\
\hline STORY29 & $\mathrm{B} 2$ & DEAD & 192.000 & 10.395 & 2118.475 \\
\hline STORY29 & $\mathrm{B} 2$ & DEAD & 216.000 & 13.207 & 1835.248 \\
\hline STORY29 & B2 & DEAD & 216.000 & 19.634 & 1819.490 \\
\hline STORY29 & B2 & DEAD & 237.000 & 22.095 & 1381.339 \\
\hline STORY29 & B2 & DEAD & 258.000 & 24.556 & 891.511 \\
\hline
\end{tabular}




\begin{tabular}{|c|c|c|c|c|c|}
\hline STORY29 & B2 & DEAD & 279.000 & 27.016 & 350.007 \\
\hline STORY29 & B2 & LIVE & 9.000 & -5.428 & 143.926 \\
\hline STORY29 & $\mathrm{B} 2$ & LIVE & 30.000 & -5.428 & 257.908 \\
\hline STORY29 & B2 & LIVE & 51.000 & -5.428 & 371.890 \\
\hline STORY29 & B2 & LIVE & 72.000 & -5.428 & 485.872 \\
\hline STORY29 & $\mathrm{B} 2$ & LIVE & 72.000 & -2.336 & 490.722 \\
\hline STORY29 & $\mathrm{B} 2$ & LIVE & 96.000 & -2.336 & 546.788 \\
\hline STORY29 & $\mathrm{B} 2$ & LIVE & 120.000 & -2.336 & 602.855 \\
\hline STORY29 & $\mathrm{B} 2$ & LIVE & 144.000 & -2.336 & 658.921 \\
\hline STORY29 & B2 & LIVE & 144.000 & 2.336 & 658.921 \\
\hline STORY29 & $\mathrm{B} 2$ & LIVE & 168.000 & 2.336 & 602.855 \\
\hline STORY29 & $\mathrm{B} 2$ & LIVE & 192.000 & 2.336 & 546.788 \\
\hline STORY29 & $\mathrm{B} 2$ & LIVE & 216.000 & 2.336 & 490.722 \\
\hline STORY29 & $\mathrm{B} 2$ & LIVE & 216.000 & 5.428 & 485.872 \\
\hline STORY29 & $\mathrm{B} 2$ & LIVE & 237.000 & 5.428 & 371.890 \\
\hline STORY29 & $\mathrm{B} 2$ & LIVE & 258.000 & 5.428 & 257.908 \\
\hline STORY29 & $\mathrm{B} 2$ & LIVE & 279.000 & 5.428 & 143.926 \\
\hline STORY29 & $\mathrm{B} 2$ & WIND & 9.000 & 22.075 & 2995.909 \\
\hline STORY29 & B2 & WIND & 30.000 & 22.075 & 2532.333 \\
\hline STORY29 & $\mathrm{B} 2$ & WIND & 51.000 & 22.075 & 2068.757 \\
\hline STORY29 & $\mathrm{B} 2$ & WIND & 72.000 & 22.075 & 1605.181 \\
\hline STORY29 & $\mathrm{B} 2$ & WIND & 72.000 & 21.980 & 1590.051 \\
\hline STORY29 & $\mathrm{B} 2$ & WIND & 96.000 & 21.980 & 1062.542 \\
\hline STORY29 & $\mathrm{B} 2$ & WIND & 120.000 & 21.980 & 535.033 \\
\hline STORY29 & $\mathrm{B} 2$ & WIND & 144.000 & 21.980 & 7.523 \\
\hline STORY29 & $\mathrm{B} 2$ & WIND & 144.000 & 21.980 & -7.523 \\
\hline STORY29 & $\mathrm{B} 2$ & WIND & 168.000 & 21.980 & -535.033 \\
\hline STORY29 & $\mathrm{B} 2$ & WIND & 192.000 & 21.980 & -1062.542 \\
\hline STORY29 & $\mathrm{B} 2$ & WIND & 216.000 & 21.980 & -1590.051 \\
\hline STORY29 & $\mathrm{B} 2$ & WIND & 216.000 & 22.075 & -1605.181 \\
\hline STORY29 & B2 & WIND & 237.000 & 22.075 & -2068.757 \\
\hline STORY29 & $\mathrm{B} 2$ & WIND & 258.000 & 22.075 & -2532.333 \\
\hline STORY29 & $\mathrm{B} 2$ & WIND & 279.000 & 22.075 & -2995.909 \\
\hline STORY28 & $\mathrm{B} 2$ & DEAD & 10.000 & $\begin{array}{l}-26.901 \\
\end{array}$ & 273.090 \\
\hline STORY28 & B2 & DEAD & 30.667 & -24.480 & 804.026 \\
\hline STORY28 & $\mathrm{B} 2$ & DEAD & 51.333 & -22.058 & 1284.914 \\
\hline STORY28 & $\mathrm{B} 2$ & DEAD & 72.000 & -19.636 & 1715.753 \\
\hline STORY28 & $\mathrm{B} 2$ & DEAD & 72.000 & -13.207 & 1731.509 \\
\hline STORY28 & $\mathrm{B} 2$ & DEAD & 96.000 & -10.395 & 2014.739 \\
\hline STORY28 & $\mathrm{B} 2$ & DEAD & 120.000 & -7.583 & 2230.474 \\
\hline STORY28 & $\mathrm{B} 2$ & DEAD & 144.000 & -4.770 & 2378.713 \\
\hline STORY28 & B2 & DEAD & 144.000 & 4.770 & 2378.713 \\
\hline STORY28 & $\mathrm{B} 2$ & DEAD & 168.000 & $7 . \overline{583}$ & 2230.474 \\
\hline STORY 28 & B2 & DEAD & 192.000 & 10.395 & 2014.739 \\
\hline STORY28 & B2 & DEAD & 216.000 & 13.207 & 1731.509 \\
\hline STORY28 & B2 & DEAD & 216.000 & 19.636 & 1715.753 \\
\hline STORY28 & $\mathrm{B} 2$ & DEAD & 236.667 & 22.058 & 1284.914 \\
\hline
\end{tabular}




\begin{tabular}{|c|c|c|c|c|c|}
\hline STORY28 & $\mathrm{B} 2$ & DEAD & 257.333 & 24.480 & 804.026 \\
\hline STORY28 & $\mathrm{B} 2$ & DEAD & 278.000 & 26.901 & 273.090 \\
\hline STORY28 & $\mathrm{B} 2$ & LIVE & 10.000 & -5.428 & 121.447 \\
\hline STORY28 & $\mathrm{B} 2$ & LIVE & 30.667 & -5.428 & 233.633 \\
\hline STORY28 & B2 & LIVE & 51.333 & -5.428 & 345.818 \\
\hline STORY28 & $\mathrm{B} 2$ & LIVE & 72.000 & -5.428 & 458.003 \\
\hline STORY28 & $\mathrm{B} 2$ & LIVE & 72.000 & -2.336 & 462.852 \\
\hline STORY28 & $\mathrm{B} 2$ & LIVE & 96.000 & -2.336 & 518.920 \\
\hline STORY28 & $\mathrm{B} 2$ & LIVE & 120.000 & -2.336 & 574.987 \\
\hline STORY28 & $\mathrm{B} 2$ & LIVE & 144.000 & -2.336 & 631.054 \\
\hline STORY28 & $\mathrm{B} 2$ & LIVE & 144.000 & 2.336 & 631.054 \\
\hline STORY28 & $\mathrm{B} 2$ & LIVE & 168.000 & 2.336 & 574.987 \\
\hline STORY28 & $\mathrm{B} 2$ & LIVE & 192.000 & 2.336 & 518.920 \\
\hline STORY28 & B2 & LIVE & 216.000 & 2.336 & 462.852 \\
\hline STORY28 & $\mathrm{B} 2$ & LIVE & 216.000 & 5.428 & 458.003 \\
\hline STORY28 & $\mathrm{B} 2$ & LIVE & 236.667 & 5.428 & 345.818 \\
\hline STORY28 & $\mathrm{B} 2$ & LIVE & 257.333 & 5.428 & 233.633 \\
\hline STORY28 & $\mathrm{B} 2$ & LIVE & 278.000 & 5.428 & 121.447 \\
\hline STORY28 & $\mathrm{B} 2$ & WIND & 10.000 & 44.028 & 5931.400 \\
\hline STORY28 & $\mathrm{B} 2$ & WIND & 30.667 & 44.028 & 5021.481 \\
\hline STORY28 & $\mathrm{B} 2$ & WIND & 51.333 & 44.028 & 4111.563 \\
\hline STORY28 & $\mathrm{B} 2$ & WIND & 72.000 & 44.028 & 3201.644 \\
\hline STORY28 & $\mathrm{B} 2$ & WIND & 72.000 & 43.840 & 3171.464 \\
\hline STORY28 & B2 & WIND & 96.000 & 43.840 & 2119.312 \\
\hline STORY28 & $\mathrm{B} 2$ & WIND & 120.000 & 43.840 & 1067.159 \\
\hline STORY28 & $\mathrm{B} 2$ & WIND & 144.000 & 43.840 & 15.006 \\
\hline STORY28 & $\mathrm{B} 2$ & WIND & 144.000 & 43.840 & -15.006 \\
\hline STORY28 & $\mathrm{B} 2$ & WIND & 168.000 & 43.840 & -1067.159 \\
\hline STORY28 & B2 & WIND & 192.000 & 43.840 & -2119.312 \\
\hline STORY28 & $\mathrm{B} 2$ & WIND & 216.000 & 43.840 & -3171.464 \\
\hline STORY28 & $\mathrm{B} 2$ & WIND & 216.000 & 44.028 & -3201.644 \\
\hline STORY28 & $\mathrm{B} 2$ & WIND & 236.667 & 44.028 & -4111.563 \\
\hline STORY28 & $\mathrm{B} 2$ & WIND & 257.333 & 44.028 & -5021.481 \\
\hline STORY28 & $\mathrm{B} 2$ & WIND & 278.000 & 44.028 & -5931.400 \\
\hline STORY27 & $\mathrm{B} 2$ & DEAD & 10.000 & -26.903 & 180.437 \\
\hline STORY27 & $\mathrm{B} 2$ & DEAD & 30.667 & -24.482 & 711.415 \\
\hline STORY27 & $\mathrm{B} 2$ & DEAD & 51.333 & -22.060 & 1192.344 \\
\hline STORY27 & $\mathrm{B} 2$ & DEAD & 72.000 & -19.638 & 1623.224 \\
\hline STORY27 & B2 & DEAD & 72.000 & -13.208 & 1638.978 \\
\hline STORY 27 & $\mathrm{~B} 2$ & DEAD & 96.000 & -10.395 & 1922.212 \\
\hline STORY27 & $\mathrm{B} 2$ & DEAD & 120.000 & -7.583 & 2137.950 \\
\hline STORY27 & $\mathrm{B} 2$ & DEAD & 144.000 & -4.771 & 2286.192 \\
\hline STORY 27 & $\mathrm{~B} 2$ & DEAD & 144.000 & 4.771 & 2286.192 \\
\hline STORY 27 & $\mathrm{~B} 2$ & DEAD & 168.000 & 7.583 & 2137.950 \\
\hline STORY 27 & $\mathrm{~B} 2$ & DEAD & 192.000 & 10.395 & 1922.212 \\
\hline STORY27 & $\mathrm{B} 2$ & DEAD & 216.000 & 13.208 & 1638.978 \\
\hline STORY27 & $\mathrm{B} 2$ & DEAD & 216.000 & 19.638 & 1623.224 \\
\hline
\end{tabular}




\begin{tabular}{|c|c|c|c|c|c|}
\hline STORY27 & B2 & DEAD & 236.667 & 22.060 & 1192.344 \\
\hline STORY27 & B2 & DEAD & 257.333 & 24.482 & 711.415 \\
\hline STORY27 & $\mathrm{B} 2$ & DEAD & 278.000 & 26.903 & 180.437 \\
\hline STORY27 & $\mathrm{B} 2$ & LIVE & 10.000 & -5.429 & 96.556 \\
\hline STORY27 & B2 & LIVE & 30.667 & -5.429 & 208.752 \\
\hline STORY27 & B2 & LIVE & 51.333 & -5.429 & 320.949 \\
\hline STORY27 & B2 & LIVE & 72.000 & -5.429 & 433.145 \\
\hline STORY27 & $\mathrm{B} 2$ & LIVE & 72.000 & -2.336 & 437.994 \\
\hline STORY27 & $\mathrm{B} 2$ & LIVE & 96.000 & -2.336 & 494.062 \\
\hline STORY27 & B2 & LIVE & 120.000 & -2.336 & 550.130 \\
\hline STORY27 & B2 & LIVE & 144.000 & -2.336 & 606.198 \\
\hline STORY27 & $\mathrm{B} 2$ & LIVE & 144.000 & 2.336 & 606.198 \\
\hline STORY27 & $\mathrm{B} 2$ & LIVE & 168.000 & 2.336 & 550.130 \\
\hline STORY27 & $\mathrm{B} 2$ & LIVE & 192.000 & 2.336 & 494.062 \\
\hline STORY27 & B2 & LIVE & 216.000 & 2.336 & 437.994 \\
\hline STORY27 & B2 & LIVE & 216.000 & 5.429 & 433.145 \\
\hline STORY27 & B2 & LIVE & 236.667 & 5.429 & 320.949 \\
\hline STORY27 & B2 & LIVE & 257.333 & 5.429 & 208.752 \\
\hline STORY27 & B2 & LIVE & 278.000 & 5.429 & 96.556 \\
\hline STORY27 & $\mathrm{B} 2$ & WIND & 10.000 & 65.628 & 8841.373 \\
\hline STORY27 & $\mathrm{B} 2$ & WIND & 30.667 & 65.628 & 7485.063 \\
\hline STORY27 & $\mathrm{B} 2$ & WIND & 51.333 & 65.628 & 6128.753 \\
\hline STORY27 & $\mathrm{B} 2$ & WIND & 72.000 & 65.628 & 4772.443 \\
\hline STORY27 & B2 & WIND & 72.000 & 65.348 & 4727.456 \\
\hline STORY27 & $\mathrm{B} 2$ & WIND & 96.000 & 65.348 & 3159.093 \\
\hline STORY27 & B2 & WIND & 120.000 & 65.348 & 1590.731 \\
\hline STORY27 & $\mathrm{B} 2$ & WIND & 144.000 & 65.348 & 22.368 \\
\hline STORY27 & $\mathrm{B} 2$ & WIND & 144.000 & 65.348 & -22.368 \\
\hline STORY27 & B2 & WIND & 168.000 & 65.348 & -1590.731 \\
\hline STORY27 & $\mathrm{B} 2$ & WIND & 192.000 & 65.348 & -3159.093 \\
\hline STORY27 & $\mathrm{B} 2$ & WIND & 216.000 & 65.348 & -4727.456 \\
\hline STORY 27 & $\mathrm{~B} 2$ & WIND & 216.000 & 65.628 & -4772.443 \\
\hline STORY27 & $\mathrm{B} 2$ & WIND & 236.667 & 65.628 & -6128.753 \\
\hline STORY 27 & $\mathrm{~B} 2$ & WIND & 257.333 & 65.628 & -7485.063 \\
\hline STORY27 & $\mathrm{B} 2$ & WIND & 278.000 & 65.628 & -8841.373 \\
\hline STORY 26 & B2 & DEAD & 12.000 & -26.672 & 94.125 \\
\hline STORY26 & B2 & DEAD & 32.000 & -24.328 & 604.128 \\
\hline STORY26 & B2 & DEAD & 52.000 & -21.985 & 1067.259 \\
\hline STORY26 & B2 & DEAD & 72.000 & -19.641 & 1483.518 \\
\hline STORY26 & $\mathrm{B} 2$ & DEAD & 72.000 & -13.208 & 1499.271 \\
\hline STORY26 & $\mathrm{B} 2$ & DEAD & 96.000 & -10.395 & 1782.509 \\
\hline STORY26 & $\mathrm{B} 2$ & DEAD & 120.000 & -7.583 & 1998.252 \\
\hline STORY26 & $\mathrm{B} 2$ & DEAD & 144.000 & -4.771 & 2146.499 \\
\hline STORY26 & $\mathrm{B} 2$ & DEAD & 144.000 & 4.771 & 2146.499 \\
\hline STORY26 & $\mathrm{B} 2$ & DEAD & 168.000 & 7.583 & 1998.252 \\
\hline STORY26 & B2 & DEAD & 192.000 & 10.395 & 1782.509 \\
\hline STORY26 & $\mathrm{B} 2$ & DEAD & 216.000 & 13.208 & 1499.271 \\
\hline
\end{tabular}




\begin{tabular}{|c|c|c|c|c|c|}
\hline STORY26 & B2 & DEAD & 216.000 & 19.641 & 1483.518 \\
\hline STORY26 & $\mathrm{B} 2$ & DEAD & 236.000 & 21.985 & 1067.259 \\
\hline STORY26 & B2 & DEAD & 256.000 & 24.328 & 604.128 \\
\hline STORY26 & B2 & DEAD & 276.000 & 26.672 & 94.125 \\
\hline STORY 26 & B2 & LIVE & 12.000 & -5.430 & 69.834 \\
\hline STORY26 & B2 & LIVE & 32.000 & -5.430 & 178.427 \\
\hline STORY26 & B2 & LIVE & 52.000 & -5.430 & 287.020 \\
\hline STORY26 & B2 & LIVE & 72.000 & -5.430 & 395.613 \\
\hline STORY26 & B2 & LIVE & 72.000 & -2.336 & 400.461 \\
\hline STORY26 & B2 & LIVE & 96.000 & -2.336 & 456.531 \\
\hline STORY26 & B2 & LIVE & 120.000 & -2.336 & 512.600 \\
\hline STORY26 & B2 & LIVE & 144.000 & -2.336 & 568.670 \\
\hline STORY26 & B2 & LIVE & 144.000 & 2.336 & 568.670 \\
\hline STORY26 & $\mathrm{B} 2$ & LIVE & 168.000 & 2.336 & 512.600 \\
\hline STORY26 & $\mathrm{B} 2$ & LIVE & 192.000 & 2.336 & 456.531 \\
\hline STORY26 & B2 & LIVE & 216.000 & 2.336 & 400.461 \\
\hline STORY26 & $\mathrm{B} 2$ & LIVE & 216.000 & 5.430 & 395.613 \\
\hline STORY26 & B2 & LIVE & 236.000 & 5.430 & 287.020 \\
\hline STORY26 & B2 & LIVE & 256.000 & 5.430 & 178.427 \\
\hline STORY26 & B2 & LIVE & 276.000 & 5.430 & $\begin{array}{l}69.834 \\
\end{array}$ \\
\hline STORY26 & B2 & WIND & 12.000 & 87.154 & 11567.400 \\
\hline STORY26 & $\mathrm{B} 2$ & WIND & 32.000 & 87.154 & 9824.320 \\
\hline STORY26 & B2 & WIND & 52.000 & 87.154 & 8081.237 \\
\hline STORY26 & $\mathrm{B} 2$ & WIND & 72.000 & 87.154 & 6338.153 \\
\hline STORY26 & $\mathrm{B} 2$ & WIND & 72.000 & 86.787 & 6278.401 \\
\hline STORY26 & B2 & WIND & 96.000 & 86.787 & 4195.503 \\
\hline STORY26 & $\mathrm{B} 2$ & WIND & 120.000 & 86.787 & 2112.605 \\
\hline STORY26 & B2 & WIND & 144.000 & 86.787 & 29.707 \\
\hline STORY26 & $\mathrm{B} 2$ & WIND & 144.000 & 86.787 & -29.707 \\
\hline STORY26 & $\mathrm{B} 2$ & WIND & 168.000 & 86.787 & -2112.605 \\
\hline STORY26 & $\mathrm{B} 2$ & WIND & 192.000 & 86.787 & -4195.503 \\
\hline STORY26 & B2 & WIND & 216.000 & 86.787 & -6278.401 \\
\hline STORY26 & B2 & WIND & 216.000 & 87.154 & -6338.153 \\
\hline STORY26 & B2 & WIND & 236.000 & 87.154 & -8081.237 \\
\hline STORY26 & B2 & WIND & 256.000 & 87.154 & -9824.320 \\
\hline STORY26 & B2 & WIND & 276.000 & 87.154 & -11567.400 \\
\hline STORY25 & B2 & DEAD & 12.000 & -26.675 & -28.544 \\
\hline STORY25 & B2 & DEAD & 32.000 & -24.331 & 481.512 \\
\hline STORY 25 & B2 & DEAD & 52.000 & -21.987 & 944.695 \\
\hline STORY 25 & B2 & DEAD & 72.000 & -19.644 & 1361.007 \\
\hline STORY25 & B2 & DEAD & 72.000 & -13.208 & 1376.757 \\
\hline STORY25 & B2 & DEAD & 96.000 & -10.396 & 1660.000 \\
\hline STORY25 & B2 & DEAD & 120.000 & -7.583 & 1875.747 \\
\hline STORY25 & $\mathrm{B} 2$ & DEAD & 144.000 & -4.771 & 2023.998 \\
\hline STORY 25 & B2 & DEAD & 144.000 & 4.771 & 2023.998 \\
\hline STORY25 & B2 & DEAD & 168.000 & 7.583 & 1875.747 \\
\hline STORY25 & B2 & DEAD & 192.000 & 10.396 & 1660.000 \\
\hline
\end{tabular}




\begin{tabular}{|c|c|c|c|c|c|}
\hline STORY25 & $\mathrm{B} 2$ & DEAD & 216.000 & 13.208 & 1376.757 \\
\hline STORY25 & $\mathrm{B} 2$ & DEAD & 216.000 & 19.644 & 1361.007 \\
\hline STORY25 & $\mathrm{B} 2$ & DEAD & 236.000 & 21.987 & 944.695 \\
\hline STORY25 & $\mathrm{B} 2$ & DEAD & 256.000 & 24.331 & 481.512 \\
\hline STORY25 & B2 & DEAD & 276.000 & 26.675 & -28.544 \\
\hline STORY25 & $\mathrm{B} 2$ & LIVE & 12.000 & -5.430 & 36.879 \\
\hline STORY25 & $\mathrm{B} 2$ & LIVE & 32.000 & -5.430 & 145.486 \\
\hline STORY25 & $\mathrm{B} 2$ & LIVE & 52.000 & -5.430 & 254.093 \\
\hline STORY25 & B2 & LIVE & 72.000 & -5.430 & 362.700 \\
\hline STORY25 & $\mathrm{B} 2$ & LIVE & 72.000 & -2.336 & 367.548 \\
\hline STORY25 & $\mathrm{B} 2$ & LIVE & 96.000 & -2.336 & 423.619 \\
\hline STORY25 & B2 & LIVE & 120.000 & -2.336 & 479.689 \\
\hline STORY25 & $\mathrm{B} 2$ & LIVE & 144.000 & -2.336 & 535.760 \\
\hline STORY25 & $\mathrm{B} 2$ & LIVE & 144.000 & 2.336 & 535.760 \\
\hline STORY25 & B2 & LIVE & 168.000 & 2.336 & 479.689 \\
\hline STORY25 & $\mathrm{B} 2$ & LIVE & 192.000 & 2.336 & 423.619 \\
\hline STORY25 & B2 & LIVE & 216.000 & 2.336 & 367.548 \\
\hline STORY25 & B2 & LIVE & 216.000 & 5.430 & 362.700 \\
\hline STORY25 & $\mathrm{B} 2$ & LIVE & 236.000 & 5.430 & 254.093 \\
\hline STORY25 & $\mathrm{B} 2$ & LIVE & 256.000 & 5.430 & 145.486 \\
\hline STORY25 & $\mathrm{B} 2$ & LIVE & 276.000 & 5.430 & 36.879 \\
\hline STORY25 & $\mathrm{B} 2$ & WIND & 12.000 & 108.352 & 14381.190 \\
\hline STORY25 & $\mathrm{B} 2$ & WIND & 32.000 & 108.352 & 12214.140 \\
\hline STORY25 & B2 & WIND & 52.000 & 108.352 & 10047.100 \\
\hline STORY25 & $\mathrm{B} 2$ & WIND & 72.000 & 108.352 & 7880.053 \\
\hline STORY25 & $\mathrm{B} 2$ & WIND & 72.000 & 107.900 & 7805.760 \\
\hline STORY25 & B2 & WIND & 96.000 & 107.900 & 5216.151 \\
\hline STORY25 & B2 & WIND & 120.000 & 107.900 & 2626.543 \\
\hline STORY25 & $\mathrm{B} 2$ & WIND & 144.000 & 107.900 & 36.934 \\
\hline STORY25 & B2 & WIND & 144.000 & 107.900 & -36.934 \\
\hline STORY25 & $\mathrm{B} 2$ & WIND & 168.000 & 107.900 & -2626.543 \\
\hline STORY25 & $\mathrm{B} 2$ & WIND & 192.000 & 107.900 & -5216.151 \\
\hline STORY25 & B2 & WIND & 216.000 & 107.900 & -7805.760 \\
\hline STORY 25 & $\mathrm{~B} 2$ & WIND & 216.000 & 108.352 & -7880.053 \\
\hline STORY25 & $\mathrm{B} 2$ & WIND & 236.000 & 108.352 & -10047.100 \\
\hline STORY 25 & $\mathrm{~B} 2$ & WIND & 256.000 & 108.352 & -12214.140 \\
\hline STORY25 & $\mathrm{B} 2$ & WIND & 276.000 & 108.352 & -14381.190 \\
\hline STORY 24 & $\mathrm{~B} 2$ & DEAD & 13.000 & -26.559 & -69.052 \\
\hline STORY24 & $\mathrm{B} 2$ & DEAD & 32.667 & -24.254 & 430.611 \\
\hline STORY24 & $\mathrm{B} 2$ & DEAD & 52.333 & -21.950 & 884.951 \\
\hline STORY24 & $\mathrm{B} 2$ & DEAD & 72.000 & -19.645 & 1293.968 \\
\hline STORY24 & B2 & DEAD & 72.000 & -13.208 & 1309.718 \\
\hline STORY24 & $\mathrm{B} 2$ & DEAD & 96.000 & -10.396 & 1592.963 \\
\hline STORY24 & $\mathrm{B} 2$ & DEAD & 120.000 & -7.583 & 1808.712 \\
\hline STORY24 & B2 & DEAD & 144.000 & -4.771 & 1956.966 \\
\hline STORY24 & $\mathrm{B} 2$ & DEAD & 144.000 & 4.771 & 1956.966 \\
\hline STORY 24 & $\mathrm{~B} 2$ & DEAD & 168.000 & 7.583 & 1808.712 \\
\hline
\end{tabular}




\begin{tabular}{|c|c|c|c|c|c|}
\hline STORY24 & B2 & DEAD & 192.000 & 10.396 & 1592.963 \\
\hline STORY24 & B2 & DEAD & 216.000 & 13.208 & 1309.718 \\
\hline STORY24 & B2 & DEAD & 216.000 & 19.645 & 1293.968 \\
\hline STORY 24 & $\mathrm{~B} 2$ & DEAD & 235.667 & 21.950 & 884.951 \\
\hline STORY24 & $\mathrm{B} 2$ & DEAD & 255.333 & 24.254 & 430.611 \\
\hline STORY24 & B2 & DEAD & 275.000 & 26.559 & -69.052 \\
\hline STORY24 & B2 & LIVE & 13.000 & -5.431 & 24.276 \\
\hline STORY24 & B2 & LIVE & 32.667 & -5.431 & 131.081 \\
\hline STORY24 & $\mathrm{B} 2$ & LIVE & 52.333 & -5.431 & 237.885 \\
\hline STORY24 & $\mathrm{B} 2$ & LIVE & 72.000 & -5.431 & 344.690 \\
\hline STORY24 & $\mathrm{B} 2$ & LIVE & 72.000 & -2.336 & 349.538 \\
\hline STORY24 & $\mathrm{B} 2$ & LIVE & 96.000 & -2.336 & 405.609 \\
\hline STORY24 & $\mathrm{B} 2$ & LIVE & 120.000 & -2.336 & 461.680 \\
\hline STORY24 & $\mathrm{B} 2$ & LIVE & 144.000 & -2.336 & 517.751 \\
\hline STORY24 & $\mathrm{B} 2$ & LIVE & 144.000 & 2.336 & 517.751 \\
\hline STORY24 & $\mathrm{B} 2$ & LIVE & 168.000 & 2.336 & 461.680 \\
\hline STORY24 & $\mathrm{B} 2$ & LIVE & 192.000 & 2.336 & 405.609 \\
\hline STORY24 & $\mathrm{B} 2$ & LIVE & 216.000 & 2.336 & 349.538 \\
\hline STORY24 & $\mathrm{B} 2$ & LIVE & 216.000 & 5.431 & 344.690 \\
\hline STORY24 & $\mathrm{B} 2$ & LIVE & 235.667 & 5.431 & 237.885 \\
\hline STORY 24 & $\mathrm{~B} 2$ & LIVE & 255.333 & 5.431 & 131.081 \\
\hline STORY24 & $\mathrm{B} 2$ & LIVE & 275.000 & 5.431 & 24.276 \\
\hline STORY24 & B2 & WIND & 13.000 & 129.388 & 17044.060 \\
\hline STORY24 & B2 & WIND & 32.667 & 129.388 & 14499.430 \\
\hline STORY24 & $\mathrm{B} 2$ & WIND & 52.333 & 129.388 & 11954.790 \\
\hline STORY24 & $\mathrm{B} 2$ & WIND & 72.000 & 129.388 & 9410.159 \\
\hline STORY24 & $\mathrm{B} 2$ & WIND & 72.000 & 128.852 & 9321.437 \\
\hline STORY24 & $\mathrm{B} 2$ & WIND & 96.000 & 128.852 & 6228.993 \\
\hline STORY24 & B2 & WIND & 120.000 & 128.852 & 3136.550 \\
\hline STORY24 & $\mathrm{B} 2$ & WIND & 144.000 & 128.852 & 44.107 \\
\hline STORY24 & $\mathrm{B} 2$ & WIND & 144.000 & 128.852 & -44.107 \\
\hline STORY24 & $\mathrm{B} 2$ & WIND & 168.000 & 128.852 & -3136.550 \\
\hline STORY24 & B2 & WIND & 192.000 & 128.852 & -6228.993 \\
\hline STORY24 & $\mathrm{B} 2$ & WIND & 216.000 & 128.852 & -9321.437 \\
\hline STORY24 & B2 & WIND & 216.000 & 129.388 & -9410.159 \\
\hline STORY 24 & $\mathrm{~B} 2$ & WIND & 235.667 & 129.388 & -11954.790 \\
\hline STORY 24 & $\mathrm{~B} 2$ & WIND & 255.333 & 129.388 & -14499.430 \\
\hline STORY24 & $\mathrm{B} 2$ & WIND & 275.000 & 129.388 & -17044.060 \\
\hline STORY23 & $\mathrm{B} 2$ & DEAD & 13.000 & -26.560 & -133.690 \\
\hline STORY23 & $\mathrm{B} 2$ & DEAD & 32.667 & -24.256 & 366.000 \\
\hline STORY23 & $\mathrm{B} 2$ & DEAD & 52.333 & -21.951 & 820.367 \\
\hline STORY23 & $\mathrm{B} 2$ & DEAD & 72.000 & -19.647 & 1229.411 \\
\hline STORY23 & $\mathrm{B} 2$ & DEAD & 72.000 & -13.208 & 1245.160 \\
\hline STORY23 & $\mathrm{B} 2$ & DEAD & 96.000 & -10.396 & 1528.407 \\
\hline STORY23 & $\mathrm{B} 2$ & DEAD & 120.000 & -7.583 & 1744.159 \\
\hline STORY23 & $\mathrm{B} 2$ & DEAD & 144.000 & -4.771 & 1892.414 \\
\hline STORY23 & $\mathrm{B} 2$ & DEAD & 144.000 & 4.771 & 1892.414 \\
\hline
\end{tabular}




\begin{tabular}{|c|c|c|c|c|c|}
\hline STORY23 & B2 & DEAD & 168.000 & 7.583 & 1744.159 \\
\hline STORY23 & B2 & DEAD & 192.000 & 10.396 & 1528.407 \\
\hline STORY23 & $\mathrm{B} 2$ & DEAD & 216.000 & 13.208 & 1245.160 \\
\hline STORY23 & B2 & DEAD & 216.000 & 19.647 & 1229.411 \\
\hline STORY23 & $\mathrm{B} 2$ & DEAD & 235.667 & 21.951 & 820.367 \\
\hline STORY23 & B2 & DEAD & 255.333 & 24.256 & 366.000 \\
\hline STORY23 & $\mathrm{B} 2$ & DEAD & 275.000 & 26.560 & -133.690 \\
\hline STORY23 & B2 & LIVE & 13.000 & -5.431 & 6.911 \\
\hline STORY23 & $\mathrm{B} 2$ & LIVE & 32.667 & -5.431 & 113.723 \\
\hline STORY23 & $\mathrm{B} 2$ & LIVE & 52.333 & -5.431 & 220.535 \\
\hline STORY23 & $\mathrm{B} 2$ & LIVE & 72.000 & -5.431 & 327.347 \\
\hline STORY23 & $\mathrm{B} 2$ & LIVE & 72.000 & -2.336 & 332.194 \\
\hline STORY23 & B2 & LIVE & 96.000 & -2.336 & 388.266 \\
\hline STORY23 & B2 & LIVE & 120.000 & -2.336 & 444.338 \\
\hline STORY23 & $\mathrm{B} 2$ & LIVE & 144.000 & -2.336 & 500.410 \\
\hline STORY23 & $\mathrm{B} 2$ & LIVE & 144.000 & 2.336 & 500.410 \\
\hline STORY23 & B2 & LIVE & 168.000 & 2.336 & 444.338 \\
\hline STORY23 & $\mathrm{B} 2$ & LIVE & 192.000 & 2.336 & 388.266 \\
\hline STORY23 & $\mathrm{B} 2$ & LIVE & 216.000 & 2.336 & 332.194 \\
\hline STORY23 & $\mathrm{B} 2$ & LIVE & 216.000 & 5.431 & 327.347 \\
\hline STORY23 & $\mathrm{B} 2$ & LIVE & 235.667 & 5.431 & 220.535 \\
\hline STORY23 & $\mathrm{B} 2$ & LIVE & 255.333 & 5.431 & 113.723 \\
\hline STORY23 & $\mathrm{B} 2$ & LIVE & 275.000 & 5.431 & 6.911 \\
\hline STORY23 & $\mathrm{B} 2$ & WIND & 13.000 & 150.257 & 19793.230 \\
\hline STORY23 & $\mathrm{B} 2$ & WIND & 32.667 & 150.257 & 16838.190 \\
\hline STORY23 & $\mathrm{B} 2$ & WIND & 52.333 & 150.257 & 13883.140 \\
\hline STORY23 & $\mathrm{B} 2$ & WIND & 72.000 & 150.257 & 10928.100 \\
\hline STORY23 & B2 & WIND & 72.000 & 149.637 & 10825.060 \\
\hline STORY23 & $\mathrm{B} 2$ & WIND & 96.000 & 149.637 & 7233.780 \\
\hline STORY23 & $\mathrm{B} 2$ & WIND & 120.000 & 149.637 & 3642.501 \\
\hline STORY23 & B2 & WIND & 144.000 & 149.637 & 51.222 \\
\hline STORY23 & $\mathrm{B} 2$ & WIND & 144.000 & 149.637 & -51.222 \\
\hline STORY23 & $\mathrm{B} 2$ & WIND & 168.000 & 149.637 & -3642.501 \\
\hline STORY23 & B2 & WIND & 192.000 & 149.637 & -7233.780 \\
\hline STORY23 & $\mathrm{B} 2$ & WIND & 216.000 & 149.637 & -10825.060 \\
\hline STORY23 & $\mathrm{B} 2$ & WIND & 216.000 & 150.257 & -10928.100 \\
\hline STORY23 & $\mathrm{B} 2$ & WIND & 235.667 & 150.257 & -13883.140 \\
\hline STORY 23 & $\mathrm{~B} 2$ & WIND & 255.333 & 150.257 & -16838.190 \\
\hline STORY23 & $\mathrm{B} 2$ & WIND & 275.000 & 150.257 & -19793.230 \\
\hline STORY22 & $\mathrm{B} 2$ & DEAD & 14.000 & -26.444 & -168.167 \\
\hline STORY22 & B2 & DEAD & 33.333 & -24.179 & 321.190 \\
\hline STORY22 & $\mathrm{B} 2$ & DEAD & 52.667 & -21.913 & 766.749 \\
\hline STORY22 & $\mathrm{B} 2$ & DEAD & 72.000 & -19.648 & 1168.508 \\
\hline STORY 22 & $\mathrm{~B} 2$ & DEAD & 72.000 & -13.208 & 1184.255 \\
\hline STORY22 & $\mathrm{B} 2$ & DEAD & 96.000 & -10.396 & 1467.505 \\
\hline STORY22 & $\mathrm{B} 2$ & DEAD & 120.000 & -7.584 & 1683.259 \\
\hline STORY22 & $\mathrm{B} 2$ & DEAD & 144.000 & -4.771 & 1831.517 \\
\hline
\end{tabular}




\begin{tabular}{|c|c|c|c|c|c|}
\hline STORY22 & B2 & DEAD & 144.000 & 4.771 & 1831.517 \\
\hline STORY22 & $\mathrm{B} 2$ & DEAD & 168.000 & 7.584 & 1683.259 \\
\hline STORY22 & B2 & DEAD & 192.000 & 10.396 & 1467.505 \\
\hline STORY 22 & $\mathrm{~B} 2$ & DEAD & 216.000 & 13.208 & 1184.255 \\
\hline STORY22 & $\mathrm{B} 2$ & DEAD & 216.000 & 19.648 & 1168.508 \\
\hline STORY22 & $\mathrm{B} 2$ & DEAD & 235.333 & 21.913 & 766.749 \\
\hline STORY 22 & $\mathrm{~B} 2$ & DEAD & 254.667 & 24.179 & 321.190 \\
\hline STORY22 & $\mathrm{B} 2$ & DEAD & 274.000 & 26.444 & -168.167 \\
\hline STORY22 & B2 & LIVE & 14.000 & -5.431 & -4.040 \\
\hline STORY22 & $\mathrm{B} 2$ & LIVE & 33.333 & -5.431 & 100.968 \\
\hline STORY22 & $\mathrm{B} 2$ & LIVE & 52.667 & -5.431 & 205.977 \\
\hline STORY22 & $\mathrm{B} 2$ & LIVE & 72.000 & -5.431 & 310.985 \\
\hline STORY22 & $\mathrm{B} 2$ & LIVE & 72.000 & -2.336 & 315.832 \\
\hline STORY22 & B2 & LIVE & 96.000 & -2.336 & 371.905 \\
\hline STORY22 & $\mathrm{B} 2$ & LIVE & 120.000 & -2.336 & 427.977 \\
\hline STORY22 & B2 & LIVE & 144.000 & -2.336 & 484.049 \\
\hline STORY22 & $\mathrm{B} 2$ & LIVE & 144.000 & 2.336 & 484.049 \\
\hline STORY22 & $\mathrm{B} 2$ & LIVE & 168.000 & 2.336 & 427.977 \\
\hline STORY22 & $\mathrm{B} 2$ & LIVE & 192.000 & 2.336 & 371.905 \\
\hline STORY22 & B2 & LIVE & 216.000 & 2.336 & 315.832 \\
\hline STORY22 & B2 & LIVE & 216.000 & 5.431 & 310.985 \\
\hline STORY22 & $\mathrm{B} 2$ & LIVE & 235.333 & 5.431 & 205.977 \\
\hline STORY22 & $\mathrm{B} 2$ & LIVE & 254.667 & 5.431 & 100.968 \\
\hline STORY22 & $\mathrm{B} 2$ & LIVE & 274.000 & 5.431 & -4.040 \\
\hline STORY22 & $\mathrm{B} 2$ & WIND & 14.000 & 170.753 & 22322.790 \\
\hline STORY22 & $\mathrm{B} 2$ & WIND & 33.333 & 170.753 & 19021.560 \\
\hline STORY22 & $\mathrm{B} 2$ & WIND & 52.667 & 170.753 & 15720.330 \\
\hline STORY22 & $\mathrm{B} 2$ & WIND & 72.000 & 170.753 & 12419.100 \\
\hline STORY22 & $\mathrm{B} 2$ & WIND & 72.000 & 170.053 & 12302.000 \\
\hline STORY22 & $\mathrm{B} 2$ & WIND & 96.000 & 170.053 & 8220.734 \\
\hline STORY22 & B2 & WIND & 120.000 & 170.053 & 4139.472 \\
\hline STORY22 & $\mathrm{B} 2$ & WIND & 144.000 & 170.053 & 58.211 \\
\hline STORY22 & $\mathrm{B} 2$ & WIND & 144.000 & 170.053 & -58.211 \\
\hline STORY22 & $\mathrm{B} 2$ & WIND & 168.000 & 170.053 & -4139.472 \\
\hline STORY22 & $\mathrm{B} 2$ & WIND & 192.000 & 170.053 & -8220.734 \\
\hline STORY22 & $\mathrm{B} 2$ & WIND & 216.000 & 170.053 & -12302.000 \\
\hline STORY22 & $\mathrm{B} 2$ & WIND & 216.000 & 170.753 & -12419.100 \\
\hline STORY22 & $\mathrm{B} 2$ & WIND & 235.333 & 170.753 & -15720.330 \\
\hline STORY22 & $\mathrm{B} 2$ & WIND & 254.667 & 170.753 & -19021.560 \\
\hline STORY 22 & $\mathrm{~B} 2$ & WIND & 274.000 & 170.753 & -22322.790 \\
\hline STORY21 & $\mathrm{B} 2$ & DEAD & 15.000 & -26.330 & -281.020 \\
\hline STORY21 & $\mathrm{B} 2$ & DEAD & 34.000 & -24.104 & 198.101 \\
\hline STORY21 & $\mathrm{B} 2$ & DEAD & 53.000 & -21.877 & 634.921 \\
\hline STORY21 & $\mathrm{B} 2$ & DEAD & 72.000 & -19.651 & 1029.439 \\
\hline STORY21 & $\mathrm{B} 2$ & DEAD & 72.000 & -13.208 & 1045.184 \\
\hline STORY21 & $\mathrm{B} 2$ & DEAD & 96.000 & -10.396 & 1328.439 \\
\hline STORY 21 & B2 & DEAD & 120.000 & -7.584 & 1544.197 \\
\hline
\end{tabular}




\begin{tabular}{|c|c|c|c|c|c|}
\hline STORY21 & $\mathrm{B} 2$ & DEAD & 144.000 & -4.771 & 1692.460 \\
\hline STORY21 & $\mathrm{B} 2$ & DEAD & 144.000 & 4.771 & 1692.460 \\
\hline STORY21 & B2 & DEAD & 168.000 & 7.584 & 1544.197 \\
\hline STORY 21 & $\mathrm{~B} 2$ & DEAD & 192.000 & 10.396 & 1328.439 \\
\hline STORY21 & $\mathrm{B} 2$ & DEAD & 216.000 & 13.208 & 1045.184 \\
\hline STORY21 & $\mathrm{B} 2$ & DEAD & 216.000 & 19.651 & 1029.439 \\
\hline STORY21 & $\mathrm{B} 2$ & DEAD & 235.000 & 21.877 & 634.921 \\
\hline STORY21 & $\mathrm{B} 2$ & DEAD & 254.000 & 24.104 & 198.101 \\
\hline STORY21 & B2 & DEAD & 273.000 & 26.330 & -281.020 \\
\hline STORY21 & $\mathrm{B} 2$ & LIVE & 15.000 & -5.432 & -36.015 \\
\hline STORY21 & B2 & LIVE & 34.000 & -5.432 & 67.198 \\
\hline STORY21 & $\mathrm{B} 2$ & LIVE & 53.000 & -5.432 & 170.411 \\
\hline STORY21 & $\mathrm{B} 2$ & LIVE & 72.000 & -5.432 & 273.624 \\
\hline STORY21 & $\mathrm{B} 2$ & LIVE & 72.000 & -2.336 & 278.471 \\
\hline STORY21 & $\mathrm{B} 2$ & LIVE & 96.000 & -2.336 & 334.544 \\
\hline STORY21 & $\mathrm{B} 2$ & LIVE & 120.000 & -2.336 & 390.618 \\
\hline STORY21 & B2 & LIVE & 144.000 & -2.336 & 446.692 \\
\hline STORY21 & $\mathrm{B} 2$ & LIVE & 144.000 & 2.336 & 446.692 \\
\hline STORY21 & $\mathrm{B} 2$ & LIVE & 168.000 & 2.336 & 390.618 \\
\hline STORY21 & $\mathrm{B} 2$ & LIVE & 192.000 & 2.336 & 334.544 \\
\hline STORY21 & B2 & LIVE & 216.000 & 2.336 & 278.471 \\
\hline STORY21 & B2 & LIVE & 216.000 & 5.432 & 273.624 \\
\hline STORY21 & $\mathrm{B} 2$ & LIVE & 235.000 & 5.432 & 170.411 \\
\hline STORY21 & $\mathrm{B} 2$ & LIVE & 254.000 & 5.432 & 67.198 \\
\hline STORY21 & $\mathrm{B} 2$ & LIVE & 273.000 & 5.432 & -36.015 \\
\hline STORY21 & $\mathrm{B} 2$ & WIND & 15.000 & 191.061 & 24787.210 \\
\hline STORY21 & $\mathrm{B} 2$ & WIND & 34.000 & 191.061 & 21157.050 \\
\hline STORY21 & $\mathrm{B} 2$ & WIND & 53.000 & 191.061 & 17526.900 \\
\hline STORY21 & B2 & WIND & 72.000 & 191.061 & 13896.750 \\
\hline STORY21 & $\mathrm{B} 2$ & WIND & 72.000 & 190.286 & 13765.700 \\
\hline STORY21 & $\mathrm{B} 2$ & WIND & 96.000 & 190.286 & 9198.846 \\
\hline STORY21 & $\mathrm{B} 2$ & WIND & 120.000 & 190.286 & 4631.992 \\
\hline STORY21 & $\mathrm{B} 2$ & WIND & 144.000 & 190.286 & 65.138 \\
\hline STORY21 & B2 & WIND & 144.000 & 190.286 & -65.138 \\
\hline STORY21 & $\mathrm{B} 2$ & WIND & 168.000 & 190.286 & -4631.992 \\
\hline STORY21 & $\mathrm{B} 2$ & WIND & 192.000 & 190.286 & -9198.846 \\
\hline STORY21 & $\mathrm{B} 2$ & WIND & 216.000 & 190.286 & -13765.700 \\
\hline STORY21 & $\mathrm{B} 2$ & WIND & 216.000 & 191.061 & -13896.750 \\
\hline STORY21 & $\mathrm{B} 2$ & WIND & 235.000 & 191.061 & -17526.900 \\
\hline STORY21 & B2 & WIND & 254.000 & 191.061 & -21157.050 \\
\hline STORY21 & $\mathrm{B} 2$ & WIND & 273.000 & 191.061 & -24787.210 \\
\hline STORY20 & $\mathrm{B} 2$ & DEAD & 15.000 & -26.331 & -332.470 \\
\hline STORY20 & $\mathrm{B} 2$ & DEAD & 34.000 & -24.105 & 146.673 \\
\hline STORY20 & $\mathrm{B} 2$ & DEAD & 53.000 & -21.878 & 583.514 \\
\hline STORY 20 & $\mathrm{~B} 2$ & DEAD & 72.000 & -19.652 & 978.052 \\
\hline STORY 20 & $\mathrm{~B} 2$ & DEAD & 72.000 & -13.209 & 993.797 \\
\hline STORY20 & $\mathrm{B} 2$ & DEAD & 96.000 & -10.396 & 1277.053 \\
\hline
\end{tabular}




\begin{tabular}{|c|c|c|c|c|c|}
\hline STORY 20 & B2 & DEAD & 120.000 & -7.584 & 1492.813 \\
\hline STORY20 & $\mathrm{B} 2$ & DEAD & 144.000 & -4.772 & 1641.078 \\
\hline STORY20 & B2 & DEAD & 144.000 & 4.772 & 1641.078 \\
\hline STORY20 & B2 & DEAD & 168.000 & 7.584 & 1492.813 \\
\hline STORY20 & $\mathrm{B} 2$ & DEAD & 192.000 & 10.396 & 1277.053 \\
\hline STORY20 & B2 & DEAD & 216.000 & 13.209 & 993.797 \\
\hline STORY 20 & B2 & DEAD & 216.000 & 19.652 & 978.052 \\
\hline STORY20 & B2 & DEAD & 235.000 & 21.878 & 583.514 \\
\hline STORY20 & B2 & DEAD & 254.000 & 24.105 & 146.673 \\
\hline STORY20 & B2 & DEAD & 273.000 & 26.331 & -332.470 \\
\hline STORY20 & $\mathrm{B} 2$ & LIVE & 15.000 & -5.433 & -49.837 \\
\hline STORY 20 & B2 & LIVE & 34.000 & -5.433 & 53.382 \\
\hline STORY20 & $\mathrm{B} 2$ & LIVE & 53.000 & -5.433 & 156.600 \\
\hline STORY20 & $\mathrm{B} 2$ & LIVE & 72.000 & -5.433 & 259.819 \\
\hline STORY20 & $\mathrm{B} 2$ & LIVE & 72.000 & -2.336 & 264.665 \\
\hline STORY20 & $\mathrm{B} 2$ & LIVE & 96.000 & -2.336 & 320.739 \\
\hline STORY20 & $\mathrm{B} 2$ & LIVE & 120.000 & -2.336 & 376.814 \\
\hline STORY20 & $\mathrm{B} 2$ & LIVE & 144.000 & -2.336 & 432.888 \\
\hline STORY20 & $\mathrm{B} 2$ & LIVE & 144.000 & 2.336 & 432.888 \\
\hline STORY20 & B2 & LIVE & 168.000 & 2.336 & 376.814 \\
\hline STORY20 & B2 & LIVE & 192.000 & 2.336 & 320.739 \\
\hline STORY20 & B2 & LIVE & 216.000 & 2.336 & 264.665 \\
\hline STORY20 & $\mathrm{B} 2$ & LIVE & 216.000 & 5.433 & 259.819 \\
\hline STORY20 & $\mathrm{B} 2$ & LIVE & 235.000 & 5.433 & 156.600 \\
\hline STORY20 & $\mathrm{B} 2$ & LIVE & 254.000 & 5.433 & 53.382 \\
\hline STORY20 & $\mathrm{B} 2$ & LIVE & 273.000 & 5.433 & -49.837 \\
\hline STORY20 & B2 & WIND & 15.000 & 211.409 & 27427.330 \\
\hline STORY20 & $\mathrm{B} 2$ & WIND & 34.000 & 211.409 & 23410.560 \\
\hline STORY20 & $\mathrm{B} 2$ & WIND & 53.000 & 211.409 & 19393.790 \\
\hline STORY20 & $\mathrm{B} 2$ & WIND & 72.000 & 211.409 & 15377.020 \\
\hline STORY20 & $\mathrm{B} 2$ & WIND & 72.000 & 210.555 & 15232.010 \\
\hline STORY20 & $\mathrm{B} 2$ & WIND & 96.000 & 210.555 & 10178.700 \\
\hline STORY20 & $\mathrm{B} 2$ & WIND & 120.000 & 210.555 & 5125.389 \\
\hline STORY20 & $\mathrm{B} 2$ & WIND & 144.000 & 210.555 & 72.077 \\
\hline STORY20 & $\mathrm{B} 2$ & WIND & 144.000 & 210.555 & -72.077 \\
\hline STORY20 & $\mathrm{B} 2$ & WIND & 168.000 & 210.555 & -5125.389 \\
\hline STORY 20 & $\mathrm{~B} 2$ & WIND & 192.000 & 210.555 & -10178.700 \\
\hline STORY20 & B2 & WIND & 216.000 & 210.555 & -15232.010 \\
\hline STORY 20 & B2 & WIND & 216.000 & 211.409 & -15377.020 \\
\hline STORY20 & B2 & WIND & 235.000 & 211.409 & -19393.790 \\
\hline STORY20 & $\mathrm{B} 2$ & WIND & 254.000 & 211.409 & -23410.560 \\
\hline STORY20 & B2 & WIND & 273.000 & 211.409 & -27427.330 \\
\hline STORY 19 & B2 & DEAD & 16.000 & -26.215 & -348.061 \\
\hline STORY19 & $\mathrm{B} 2$ & DEAD & 34.667 & -24.028 & 120.870 \\
\hline STORY 19 & $\mathrm{~B} 2$ & DEAD & 53.333 & -21.840 & 548.970 \\
\hline STORY19 & $\mathrm{B} 2$ & DEAD & 72.000 & -19.653 & 936.239 \\
\hline STORY 19 & $\mathrm{~B} 2$ & DEAD & 72.000 & -13.209 & 951.983 \\
\hline
\end{tabular}

